\title{
ROBUSTNESS OF SELF-TUNING CONTROLLERS
}

$$
\text { by }
$$

LIM Khiang wee

Hertford College, oxford

\section{A Thesis submitted for the degree of Doctor of Philosophy}

\section{Trinity Term 1982}

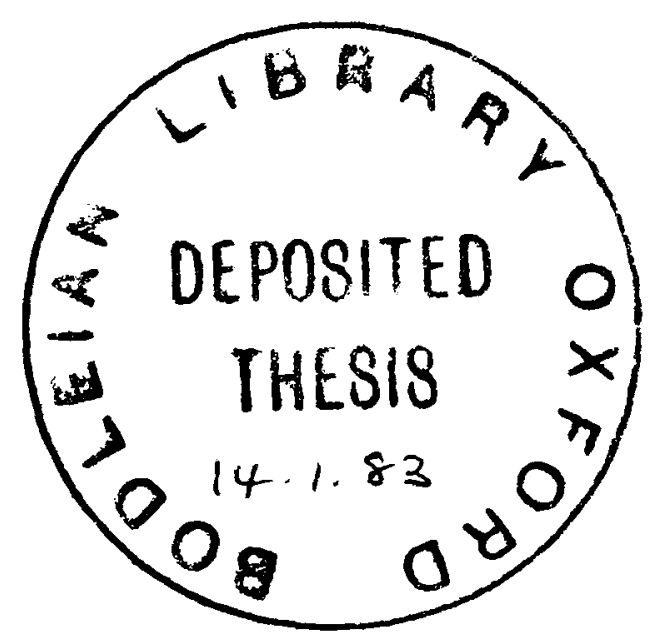

Department of Engineering science,

University of oxford,

Parks Road,

oxford, England. 


\title{
ROBUSTNESS OF SELF-TUNING CONTROLLERS
}

\author{
LIM Khiang Wee, Hertford College \\ Thesis submitted for the degree of \\ Doctor of Philosophy
}

Trinity Term 1982

\begin{abstract}
Over the last decade, considerable effort has been devoted to the implementation and analysis of self-tuning controllers on systems which are assumed to be represented exactly by linear dynamical models. In this thesis we examine the robustness of the self-tuning controller, when applied to systems consisting of a nominal linear plant which may have linear or nonlinear perturbations. Robust stability is the primary criterion and most of the results are for the clarke-Gawthrop version of the self-tuning controller. Conditions are derived for the robust stability of the adaptively controlled system in terms of the design choices available to the engineer setting up the self-tuning controller. These are strong stability results in that they are in terms of both $l_{2}$ and $l_{\infty}$ stability. The results are shown to be applicable to the general delay case and in the presence of non-zero mean disturbances. Preliminary results are also obtained for the robust stability of the explicit self-tuning controller.
\end{abstract}




\section{ACKNOWLEDGEMENTS}

I would like to thank

- Dr. P.J. Gawthrop who proposed the research and guided me through it

- Dr. D.W. Clarke for many useful discussions and for his encouragement

- All the members of the self-tuning group in oxford

- The Kuok Foundation, Malaysia for their generous sponsorship 


\section{ABSTRACT}

ACKNOWLEDGEMENTS

NOTATION

CHAPTER 1

1.1
1.2
1.3
1.4

CHAPTER 2

\section{1}

2.2

2.2 .1

2.2 .2

2.2 .3

2.3

2.3 .1

2.3 .2

2.4

2.4 .1

2.4 .2

2.4 .3

2.4 .4

2.4 .5

2.4 .6

2.5

2.5 .1

2.5 .2

2.5 .3

2.6

2.6 .1

2.6 .2

SELF-TUNING ALGORITHMS . . . . . . . . . . . . . 3

STABILITY AND CONVERGENCE . . . . . . . . . . 10

MATHEMATICAL PRELIMINARIES . . . . . . . . . 17

OUTLINE OF THESIS . . . . . . . . . . . . . . 21

REVIEW OF STANDARD STABILITY RESULTS . . . . . . . 24

MODEL OF PERTURBED SYSTEM . . . . . . . . . . . . 28

Higher Order Systems . . . . . . . . . . 29

Nonlinearities . . . . . . . . . . . . 30

Other Representations . . . . . . . . . . . 31

ERROR FEEDBACK SYSTEM . . . . . . . . . . . . 32

The Self-tuning Algorithm . . . . . . . . . 32

Error Equations . . . . . . . . . . . . . 36

ROBUST STABILITY: THE NON-ADAPTIVE CASE . . . . 38

Output Error . . . . . . . . . . . . . . 39

Input Error . . . . . . . . . . . . . . . 46

Discussion . . . . . . . . . . . . . . . . . 49

pole-Zero placement (Astrom And

Wittenmark[10],[11]) . . . . . . . . . . 52

Pole Assignment (Wellstead et al[85],[86]) . . . 55

Instability of The Non-adaptive Controller . . . 57

ROBUST DISTURBANCE ATTENUATION: NON-ADAPTIVE

CONTROLLER . . . . . . . . . . . . . . . . . 61

Feedback System And Equations . . . . . . . . 62

Theorems For Disturbance Attenuation . . . . . . 62

Setpoint Following . . . . . . . . . . . . . 66

EXAMPLES . . . . . . . . . . . . . . . . 67

Reduced Order Linear Dynamics . . . . . . . . 68

Nonlinear Example . . . . . . . . . . . 72

CHAPTER 3

3.1
3.2
3.2 .1
3.2 .2
3.3
3.4
3.4 .1
3.4 .2
3.4 .3
3.5
3.6

INPUT-OUTPUT PROPERTIES OF THE ESTIMATOR . . . . . 79 STABILITY OF LINEAR FULL ORDER CONTROLIER . . . . 84

No C Estimation (Fixed Observer) . . . . . . . 85

C Estimated . . . . . . . . . . . . . . 87

ERROR MODEL: ADAPTIVE CASE . . . . . . . . . . . 93

ROBUST STABILITY OF ADAPTIVE CASE . . . . . . . . 94

Output Error . . . . . . . . . . . . . . 96

Input Error . . . . . . . . . . . . . . 101

Examples . . . . . . . . . . . . . . . . 102

INSTABILITY OF THE ADAPTIVE ALGORITHM . . . . . . 108

EXPLICIT ALGORITHMS: STABILITY AND ROBUSTNESS • • 112 


\begin{tabular}{|c|c|c|c|c|c|c|c|c|c|c|c|}
\hline .6 .1 & special & Cases & & & & & & & & & \\
\hline 3.6 .2 & Example & . & & & & - & - & & • & & • \\
\hline 3.6 .3 & Discussic & on: Som & & & & & & & its & & \\
\hline 3.7 & - SEQUENCE & 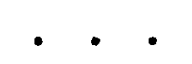 & & & - & - & 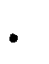 & 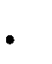 & - & & \\
\hline 3.8 & ANOTHER EST & TIMATOR & & & & • & . & • & • & & \\
\hline 3.8 .1 & Examples & . & $\bullet$ & ${ }^{\circ}$ & • & $\cdot$ & • & • & • & & \\
\hline 3.9 & DISCUSSION & . . & & • & - & . & . & . & 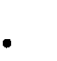 & & \\
\hline
\end{tabular}

NONADAPTIVE SELF-TUNING CONTROLLER . . . . . . 183

APPENDIX A
A. 1
SECTION 2.2: PROOF OF IEMMA 1 . . . . . . . . . . 196
A. 2 SECTION 2.3.2: DERIVATION OF EQNS. 2-34 TO 2-37 . 197
A. 3

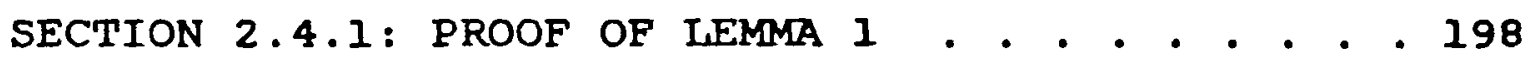
A. 4

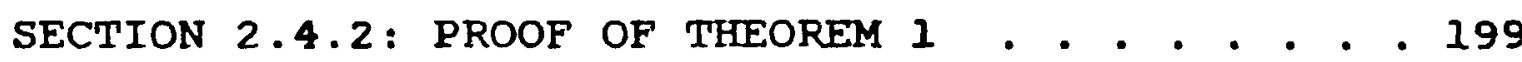
A. 5
A. 5.1
A. 5.2 SECTION 2.4 .4 . . . . . . . . . . . . . . . . . 200
A. 6
Derivation of Fig. 2-10, section 2.4.4. . . . 200
Proof of Theorem 1. . . . . . . . . . . 201 SECTION 2.4 .5 . . . . . . . . . . . . . . 202

APPENDIX B

\begin{tabular}{|c|c|c|c|c|c|c|c|c|c|c|c|c|}
\hline B. 1 & PROOFS FOR & SECTIC & & 3.1 & • & - & - & & • & - & & 203 \\
\hline B.1.1 & Proof of & Lemua & 2 & . & - & - & & & - & . & & 203 \\
\hline B. 1.2 & Proof of & Lemma & 3 & & • & - & & & . & & & 204 \\
\hline B. 1.3 & Proof of & Iemiria & 4 & . & . . & - & $\bullet^{\circ}$ & & - & • & & 205 \\
\hline B. 2 & PFOOF OF & HEOFEM & 1( & B), & SEC & & ON & & .2 .2 & • & & 205 \\
\hline B. 3 & PROOF OF I & EMMA 1 , & $S E$ & CTIOI & & & .1 & & & & & 206 \\
\hline
\end{tabular}




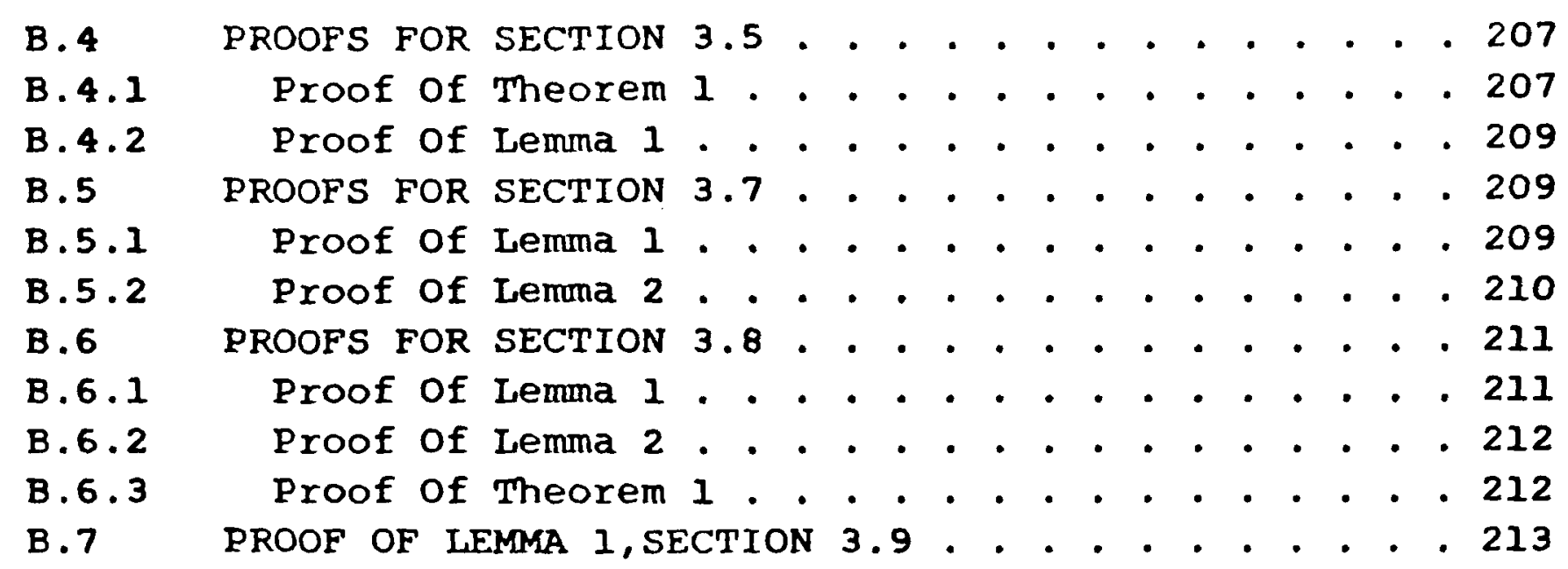

APPENDIX C

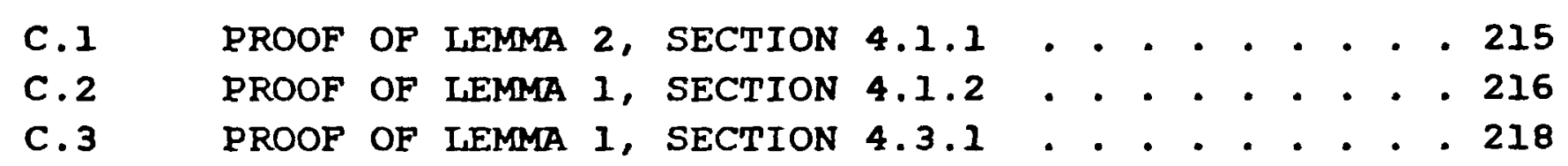


NOTATION

Equations and figures will be numbered sequentially within each chapter or appendix. When referred to from outside the chapter, the chapter number is added as a prefix eg. Eqn. 2-33.

In general, $\hat{\imath}$ will refer to an estimate of the variable $?$.

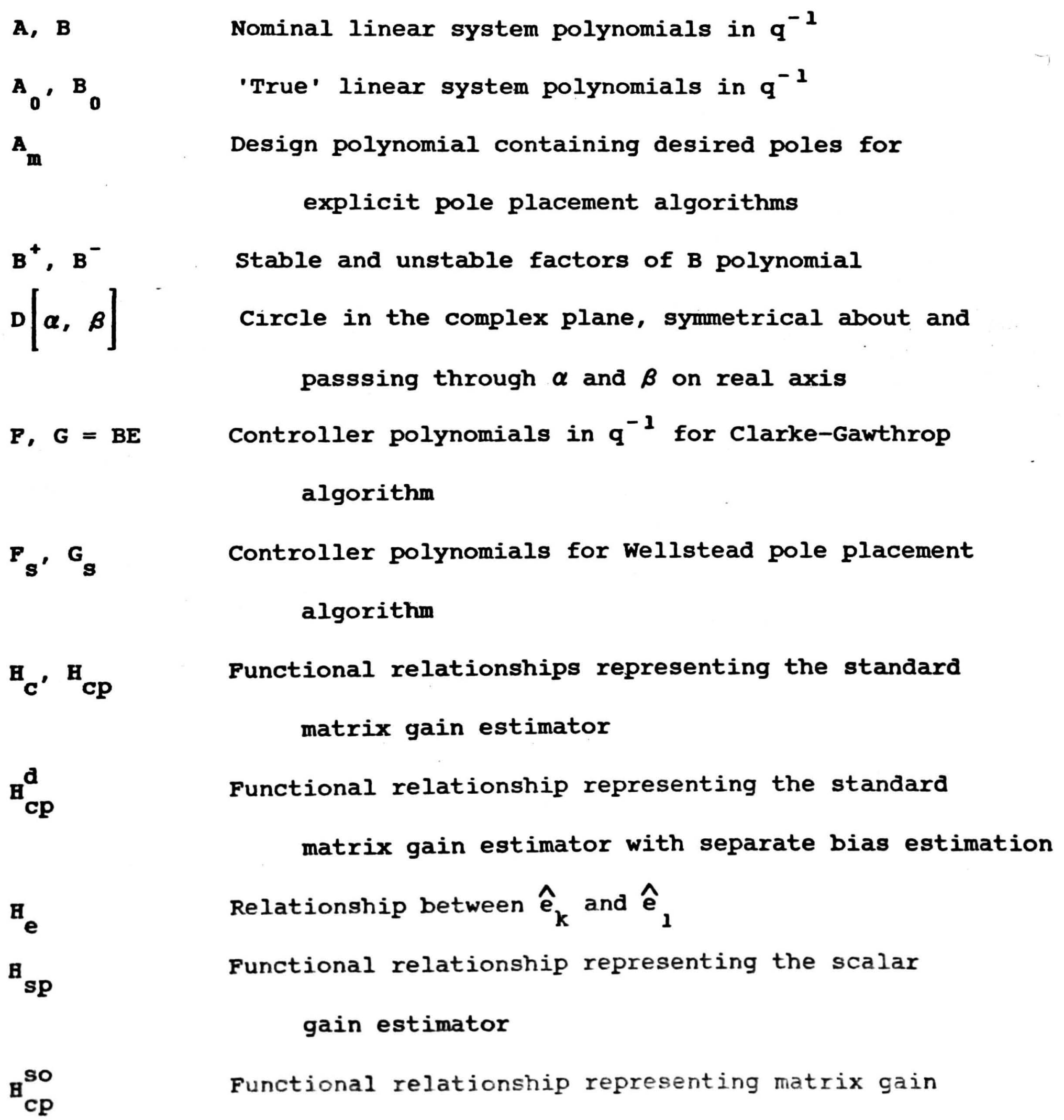


$\mathrm{H}_{\mathrm{cp}}^{\mathrm{sC}}$

$\mathrm{H}_{0}, \mathrm{H}_{\mathrm{i}}$

$\mathrm{M}_{1}, \mathrm{M}_{2}$

$P, Q, R, C$

$s(t)$

$s_{A}, R_{A}, R_{1}$

$\mathbf{X}$

$x_{0}$

$\operatorname{deg}\{$.

$d(t)$

$\overline{\mathbf{d}}$

$e(t)$

$\hat{e}_{i}$

$e_{p}(t)$

$e^{k}$

$e_{m}(t)$

k

1 estimator when used with outer loop PI

Functional relationship representing matrix gain

estimator when used with cascaded PI

Composite forward element of error feedback system

for output and input error cases

Multiplicative plant perturbations at output and input

Design polynomials/transfer functions in $\mathrm{q}^{-1}$

Matrix gain factor in least squares estimator

$=\sum^{t} \beta^{t-i} \times(i) x^{T}(i)$

Controller polynomials for Astrom's pole placement

algorithm

Filtered data vector of inputs and outputs from nominal

$$
\text { plant }=\frac{1}{c}[u(t), u(t-1), \ldots ; y(t), y(t-1) \ldots]^{T}
$$

Filtered data vector of measured inputs and outputs

$$
=\frac{l}{c}\left[u_{0}(t), u_{0}(t-1), \ldots ; y_{0}(t), y_{0}(t-1) \ldots\right]^{T}
$$

Occasionally the subscript, is dropped when it is clear which data is referred to

Degree of . polynomial in $\mathrm{q}^{-1}$

Zero mean disturbance

Mean level of disturbance

Disturbance which cannot be rejected by causal control

$$
\equiv E\left(q^{-1}\right) d(t)
$$

ith step estimated prediction error

$$
=\Phi(t)-x_{0}^{T}(t-k) \hat{\theta}(t-i)
$$

parameter $\in$ rror $\cong x_{0}^{T}(t-k) \tilde{\theta}(t-1)$

delayed parcineter error $\equiv x_{0}{ }^{T}(t-k) \bar{\theta}(t-k)$

modelling error $\triangleq\left[x_{0}(t-k)-x(t-k)\right]^{T} \theta-p \tilde{y}(t)$

Integer system delay $\geqslant 1$

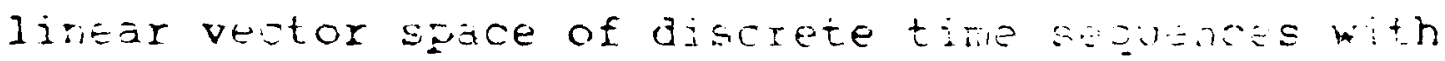


NOTATION

\section{finite ..}

$1_{2} \cdot 1_{2}^{1}$

$\mathrm{q}^{-1}$

$s(t)$

$t_{0}$

${ }^{u}, u$

$Y_{0}, Y$

$\bar{y}, \bar{u}$

$w(t)$

w.p. 1

$\boldsymbol{\omega}$

$\omega_{\mathbf{s}}$

B

$\boldsymbol{\beta}_{\text {dc }}$

$\theta$

$\theta_{p}$

$\sigma(t)$

$\sigma_{\mathbf{0}}$

T

$\Phi(t)$

$\Phi^{*}$

$\hat{\Phi}_{i}(t / t-i)$

.. (n. $\|_{\Delta}$

1.1

$E(\cdot)$

NA C-G STC

$L^{*}\left(q^{-1}\right)$
Orthogonal subspaces of $I_{2}$ which form a direct sum Backwards shift operatior i.e $q^{-1} x(t)=x(t-1)$

Trace of $s(t)$

Initial time

Inputs to real plant and linear nominal plant

Output of real plant and linear nominal plant

output and input errors

Setpoint

with probability one

Frequency in radians/second

Sampling frequency

Forgetting factor in estimator

Forgetting factor in bias estimator

Parameter vector, used in estimator

Parameter vector for explicit algorithm

$x_{0}^{T}(t) s^{-1}(t) x_{0}(t)$

$\sup _{t>t_{0}} \sigma(t)$

Fractional delay in terms of sampling period eg. 0.4T

Auxilliary output $=P\left(q^{-1}\right) Y(t)$

(Optimal) prediction for $\Phi$ if parameters of model

are known

Estimate for $\Phi^{*}(t)$ given data till time $t-i$

$1_{2}$ norm $=\left[\sum^{\infty} 1 \cdot(i) l^{2}\right]^{1 / 2}$ (incremental norm)

$I_{\infty}$ norm $=\sup _{t>t_{0}} 1.1$

Expectation of

Nonadaptive Clarke-Gawthrop self-Tuning Controller

The crerator chtajned rrom I by wejutiting all joles 


\section{CHAPTER 1}

INTRODUCTION

Self-tuning control is one form of adaptive control, based on a simple idea: The system to be controlled is represented by a linear dynamical model with constant but unknown parameters. A control law is derived as if the parameters are known. The unknown parameters are then estimated by some recursive online method and the estimates are used in the control law as if they were the true parameters.

The idea dates back to a paper published by Kalman[51] in 1957, who designed a "self-optimising controller" based on a dedicated computer. It was not until 1970, with the wider availability of computers suitable for control, that the idea was further developed for regulation, by Peterka[72] and latex by Astrom and wittenmark[8]. subsequently, clarke and Gawthrop[19],[20] generalised the self-tuner to include control weighting and setpoint following and to provide fresh interpretations.

Many papers have since been published on self-tuning controllers. Most have concentrated on the problems of algorithmic implementation and simulation of linear systems. Some, including the early work of Astrom and wittenmark[8], also showed that if the system was stable and if the parameter estimates converged, the resulting controller had some nice properties. 


\section{INTRODUCTION}

Only later did the first proofs of stability for various versions of the controller emerge. Egardt[33], Gawthrop[38], [40] and Goodwin et al[44],[49] have since shown that if the system can really be represented by the linear models assumed, their versions of the self-tuning controller will regult in a stable closed loop system. Their work is reviewed in section 1.3 .

stability is of course an essential property for any controller. However the stability proofs mentioned above are valid only when the physical plant is exactly represented by the assumed model in the self-tuning controller. It is often argued that it is impossible to model systems exactly with a linear finite dimensional model as all true systems are nonlinear and infinite dimensional. Linear models are usually approximations valid around an operating region of interest. Frrors can occur in the development of a linear model or it may simply be uneconomic to get the most accurate model. Furthermore, even if a highly accurate model is available, it may be too complicated to be used in an adaptive control algorithm.

stability by itself is thus not sufficient. We require that the self-tuning controller should also be robustly stable. Unfortunately, there are as yet few studies of self-tuning controllers which look into this problem. Some preliminary results were presented in [42].

Another property that is still a popular topic for analysis is convergence. This is usually taken to mean that the adaptive controller tends asymptotically to that which would have been obtained if the parameters of the model describing the physical plant are known. After robustness and stability, this is probably a desirable property of the 
INTRODUCTION

adaptive controller, if the assumed model does represent the plant. However, when the assumed model does not represent the plant, it is not clear what this sort of convergence means. Furthermore, most analyses to date suffer from the need to prove stability first or assume it a priori and to assume a highly structured disturbance. For these reasons, it does not appear that convergence is of much practical significance.

This thesis concentrates on a robustness study of the self-tuning controlled system when the system cannot be accurately represented by the assumed model. For analysis to be feasible we still need to assume some structure for the plant. Here we will consider two cases: the true plant is linear, but it is of higher order than the assumed system or the true plant is nonlinear. It is felt that this choice is sufficiently flexible to afford a reasonable reflection of reality and yet remain amenable to analysis. Some justification of the models used is provided in Chapter 2. Most of the results will be for the clarke-Gawthrop version of the self-tuning controller. Conditions for robust stability are developed. These are in terms of parameters used by an engineer in setting up a self-tuning controller and hence they serve as design guidelines for robustness.

\subsection{SETF-TUNING AT,GORITHMS}

In all the basic self-tuning algorithms, the system is assumed to be accurately modelled by a linear, discrete-time model with constant coefficients. For compactness, the autoregressive-moving average representation (ARMA), popularised by Box and Jenkins[16] for time series analysis is usually used. Unlike the model reference schemes which were the focus of work in adaptive control for most of the 60's, (see 
INTRODUCTION

Iandau[59] for a comprehensive survey), self-tuning control was developed in a stochastic framework to take explicit account of disturbances.

The system model is

$$
\begin{aligned}
& y(t)+a_{1} y(t-1)+\ldots+a_{n} y(t-n)=b_{0} u(t-k)+b_{1} u(t-k-1)+\ldots+ \\
& b_{n} u(t-k-n)+\xi(t)+c_{1} \xi(t-1)+\ldots+c_{n} \xi(t-n) \\
& \text { It can be written more compact]y as } \\
& A\left(q^{-1}\right) Y(t)=B\left(q^{-1}\right) u(t-k)+c\left(q^{-1}\right) \xi(t)
\end{aligned}
$$

where $A, B$ and $C$ are polynomials in the backwards shift operator, $q^{-1}$. The system order $n$ and integer delay $k$ are assumed to be known. $\xi(t)$ is a zero mean white noise process. The disturbance entering the system is thus the stationary stochastic process $\frac{C}{A} \xi(t)$ and the representation theorem (see for example Astrom[6]) is usually invoked to justify the assumption that $c\left(q^{-1}\right)$ has all its roots outside the unit circle.

The first self-tuning paper published with a detailed analysis was that of Astrom and wittenmark[8]. Their self-tuning regulator (as it was known then) was designed for a simpler version of Eqn. 2 above, with $c\left(q^{-1}\right)$ assumed to be a scalar. The control objective was to minimise the variance of the output, $\mathrm{E}\left\{\mathrm{y}^{2}\right\}$. A certainty equivalence approach was adopted in the design of the control law. If the parameters of the system are known, the minimum variance control law, Astrom[6] is obtained by first dissociating the process disturbance into unrealisable and realisable parts:

$$
\frac{C}{A} \xi(t+k)=E \xi(t+k)+\frac{P}{A} \xi(t)
$$

The first term on the RHS of Eqn. 3 has $k-1$ components and at time $t$, represents disturbances that are in the future and thus unknown, but which will affect the output at time $t+k$. Because of the delay, $k$, the 
INTRODUCTION

output at time $t+k$ is the earliest which can be modified by an input at time $t$. The second term on the RHS of Eqn. 3 represents disturbances up to time $t$, which are thus known. Using the decomposition of Fqn. 3 , the system of Fqn. 2 can be written in a predictive form as

$$
y(t+k)=\frac{F}{C} y(t)+\frac{F B}{C} u(t)+F \xi(t+k)
$$

Eqn. 4 shows that the output at time $t+k$ is a linear combination of inputs and outputs till time $t$ and a linear combination of noise in the interval from $t+1$ to $t+k$.

It is intuitively clear that the minimum variance control law would set the first two terms on the RHS of Eqn. 4 to zero, i.e.

$$
\frac{P}{C} y(t)+\frac{B E}{C} u(t)=0
$$

In the self-tuning context, the parameters of $A, B$ and $C$ and hence those of $E$ and $F$ are unknown. If $c_{1} \ldots c_{n}$ are zero, note from Eqn. I that the output $y$ can be written as a linear combination of past outputs, past inputs and the white noise term, $\xi(t)$. Some standard estimation procedure can then be used to estimate the parameters of $A\left(q^{-1}\right)$ and $B\left(q^{-1}\right)$, which could be used to solve for $E$ and $F$ in Eqn. 3. This approach subsequently became known as the explicit self-tuning approach.

Astrom and wittenmark however chose an alternative approach where the parameters of the prediction model in Eqn. 4 are estimated instead. These parameters are exactly those of the control law in Eqn. 5 and hence the computational burden of resolving the identity of Eqn.3 is avoided. This method is now known as the implicit self-tuning method. 
INTRODUCTION

with this approach and using a recursive least squares estimator, Astrom and wittenmark showed that if the parameter estimates converged, they would converge to those of the corresponding minimum variance control law.

However minimum variance regulation while satisfactory for some purposes, is too narrow a control criterion for most others. setpoint tracking may be desired or some costing may have to be imposed on the control action. Furthermore, the minimum variance control law of Eqn. 5 seeks to cancel the zeros of the system and is thus not practicable when the system has nonminimum phase zeros.

In 1975, Clarke and Gawthrop[19] generalised the self-tuning regulator, with subsequent papers providing new interpretations $[37],[20],[22]$. In this generalisation, the control law is designed to minimise the variance of an auxiliary sytem:

$$
\Phi_{T}(t)=P\left(q^{-1}\right) Y(t)+Q\left(q^{-1}\right) u(t-k)-R\left(q^{-1}\right) w(t-k)
$$

where $w(t)$ is the desired setpoint and $P, Q, R$ are design polynomials or transfer functions in $\mathrm{q}^{-1}$.

A certainty equivalent approach is also used in the control law design. Instead of attempting to predict $\Phi_{T}(t)$ however, a predictor is derived for $\Phi(t)=P y(t):$

$$
\Phi(t+k)=\frac{F}{C} Y(t)+\frac{E B}{C} u(t)+E \xi(t+k)
$$

where $E$ and $F$ are obtained from an identity similar to Eqn. 3 above.

Hence in the case of known parameters, the prediction for $\Phi_{T}(t+k)$ is

$$
\Phi_{T}{ }^{\star}(t+k / t)=\frac{F}{C} Y(t)+\frac{B E}{C} u(t)+Q u(t)-R w(t)
$$

At each time $t$, the minimum variance control law for the auxiliary process, $\Phi_{T}$ sets the RHS of Eqn. 8 above to zero. 
INTRODUCTION

This definition of an auxiliary process provides some interesting interpretations for the design polynomials. With this control law, the cloged loop system (when the parameters are known) is:

$$
\begin{aligned}
& y(t)=\frac{B R}{P B+Q A} w(t-k)+\frac{E B+Q C}{P B+Q A} \xi(t) \\
& u(t)=\frac{A R}{P B+Q A} w(t)+\frac{E A-C P}{P B+Q A} \xi(t)
\end{aligned}
$$

When $Q=0$ and $P=1$, the self-tuning controller collapses to the minimum variance controller of Astrom and Wittenmark.

When $Q=0$ and $P \neq 1$, the closed loop output is

$$
Y(t)=\frac{R}{P} w(t-k)+\frac{E}{p} \xi(t)
$$

and the input $u(t)$ is

$$
u(t)=\frac{A R}{P B} w(t)+\frac{F \cdot A-C P}{P B} \xi(t)
$$

Prom Eqn. 11, it is clear that the output attempts to follow the model $P$ in tracking the setpoint $w$. Hence $P$ will often be referred to as the reference model polynomial. Note that the output responds to the disturbances, $E \xi(t)$, with the same $\frac{1}{p}$ model. Where this is not desirable, the setpoint filter $R$, allows an additional 'degree of freedom.'

$Q\left(q^{-1}\right)$ is a form of generalised control weighting. The simplest $Q$ is a scalar $\lambda$. Some form of weighting is necessary when the system has nonminimum phase zexos, as Eqn. 12 shows that the input would become unstable otherwise. A larger scalar weighting $\lambda$ would move the poles of the system away from the roots of $P B$ to those of $\lambda A$ (assumed stable).

There is an alternative interpretation for $Q\left(q^{-1}\right)$. The control law can be written, from Eqns. 7,8 as

$$
u(t)=\frac{1}{Q}\left[\Phi^{*}(t+k / t)-R w(t)\right]
$$


INTRODUCTION

$\frac{1}{Q}$ thus acts as a compensator on the difference between the prediction for $P\left(q^{-1}\right) y$ and the filtered setpoint $R w(t)$.

From Eqns. 9,10 it is clear that since the noise is assumed to be zero mean, then for zero steady state error between the output, $y$ and the setpoint, $w$ the design parameters must also be selected to such that

$$
\frac{B(1) R(1)}{P(1) B(1)+Q(1) A(1)}=1
$$

Since $A$ and $B$ will not usually be known, this is difficult unless $Q=0$. If $Q \neq 0$, then $Q(1)$ must be chosen to be zero. A suggested choice is

$$
Q\left(q^{-1}\right)=\lambda\left(1-q^{-1}\right) /\left(1-\alpha q^{-1}\right): \lambda, \alpha(<1) \text { are scalars }
$$

Comparing with Eqn. 13, this choice of $Q$ corresponds to a proportional + integral compensator on the predicted error.

When the parameters of the system are unknown an estimator is of course needed. The clarke-Gawthrop self-tuner also uses an implicit method. Unlike the original Astrom self-tuner, $C$ is also estimated. In that case, recursive least squares will give biased estimates. Other estimation methods such as extended least squares may have to be used. Detailed estimator descriptions will be in Chapters 2 and 3 . All the estimators considered are of the form:

New estimate $=$ old estimate + gain ${ }^{2}$ prediction error of old estimate (16)

They differ mainly in the way by which the gain is updated.

The Clarke-Gawthrop self-tuner is the principal self-tuning algorithm analysed in this thesis and further details will be provided where they are needed. 
INTRODUCTION

The explicit self-tuning approach differs from that discussed above in that the controller parameters are not estimated directly, but are computed according to some design procedure, using the estimated system parameters A, B and C. Wellstead et al[85],[86] have chosen a procedure which seeks to place the closed loop poles of the controlled system. An advantage of this approach is that no attempt is made to cancel system zeros and hence it is better suited to nonminimum phase systems. It also has the advantage that it does not require an accurate knowledge of time delays, which may even be time-varying. A detailed description of this procedure can be found in section 2.4 .5 and a robustness analysis is presented in Chapter 3. Subsequent work has extended the original pole placement regulator to servo control[87]. Allidina and Hughes[1] have combined the algorithms of wellstead and clarke-Gawthrop to produce a generalised pole-shifting controller which includes control weighting and setpoint following. However, it is still an explicit method requiring the on-line resolution of a polynomial identity.

Astrom and Wittenmark[11] have also developed an explicit pole placement approach to self-tuning. Unlike wellstead, their work was focussed on the deterministic servo problem and the system is assumed to be disturbance free. The control law is more sophisticated than Wellstead's in that zeros can also be placed. It is recognised however that unstable or poorly damped zeros must not be cancelled. Hence system zeros can be factorised into those to be moved and those which are better left alone. This control law is discussed in more detail in Section 2.4.4. 
INTRODUCTION

A 'superset' of pole placement self-tuners has been developed by Clarke[18]. Many other varieties of self-tuning controllers have also been developed. Only those which will later be analysed for robustness have been outlined above. Among the latest developments are the hybrid self-tuners of Gawthrop[39] and self-tuners based on state space type system models, I,am[57], Warwick[84]. Many of the later developments have been inspired by the results of application studies. Hence refinements such as variable forgetting factors to discount past data [23], [34], feedforward[2], offset elimination [67] have been added to the basic self-tuner.

In the next section, some early work on the stability and convergence of self tuning algorithms will be reviewed. For reasons which will be clearer in Chaptex 3, nearly all these have been for implicit self-tuners.

\subsection{STABILITY AND CONVERGENCE}

In the previous section, all the self-tuning controllers reviewed were based on the certainty equivalence principle. Many of these algorithms have been verified in simulations or even actual plant trials, before proofs of the stability of these algorithms were available. In contrast, the continuous time model reference adaptive controllers reviewed by Landau[59] have nearly all been designed according to some stability criteria. However, there has been a considerable amount of recent research dedicated to showing that self-tuning algorithms are stable. A detailed review of these can be found in Gawthrop[41]. In this section, an informal summary of the important stability and convergence results will be presented. 
INTRODUCTION

The first efforts were concentrated on convergence, assuming stability. In fact, in Astrom and Wittenmark's original paper[8], two fundamental. results were established. The first characterised the results of convergence in terms of input and output covariances:

$$
\begin{aligned}
& E\{y(t+\tau) Y(t)\}=0 \tau=k+1, \ldots, k+n \\
& E\{y(t+\tau) u(t)\}=0 \tau=k+1, \ldots, n+2 k
\end{aligned}
$$

The second was a self consistency check in showing that if the parameters converge, they would converge to the minimum variance regulator which would have been obtained if the parameters of the system were known.

Ljung[63],[64],[66] then developed a general method for analysing the convergence, not only of self-tuning algorithms, but of various stochastic estimation methods. His method associates with each algorithm an ordinary differential equation (ODE). Convergence analysis for the algorithm is then reduced to stability analysis of the ODE.

For example, Astrom and Wittenmark's self-tuning regulator can be associated with the following set of coupled oDEs.

$$
\begin{aligned}
& \dot{\theta}=R_{D}^{-1}(t) G_{D}(\theta)\left[\theta_{0}-\theta\right] \\
& R_{D}=G_{D}(\theta)-R_{D}
\end{aligned}
$$

where $\theta$ is the parameter vector, $\theta_{0}$ are the true parameters and $G_{D}(\theta)=E\left\{X(t) X^{T}(t)\right\}, X$ being the data vector containing past values of inputs and outputs. 
INTRODUCTION

Isjung shows that

1. $\theta$ can converge only to stable stationary points of Eqns. 19,20

2. If $\theta$ belongs to the domain of attraction of a stable stationary point $\theta^{*}$, then $\theta$ converges w.p. 1 to $\theta^{*}$ as $t \rightarrow \infty$.

3. The trajectories of Eqns. 19,20 are the asymptotic paths of the estimator.

Using this method, Ljung showed that for the self-tuning regulator to converge, then assuming stability, a positive real condition on the noise parameters had to be satisfied. This is the now well known requirement that

$\operatorname{Re}\left\{\frac{1}{c}-\frac{1}{2}\right\}>0 \quad$ or $\quad\left|c\left(q^{-1}\right)-1\right|<1 \quad \forall|q|=1$

Ljung's method is very general, but it requires an a priori assumption of stability. An early stabilisation result was developed by Ljung and wittenmark[65] for Astrom and wittenmark's self-tuning regulator. Loosely stated, they showed that if the noise, $\xi(t)$ is sample mean square bounded, the time delay $k$ and system order $n$ are known and the first coefficient of $B\left(q^{-1}\right)$ is known, then with the appropriate self-tuning regulator, the output is also mean square bounded.

Following these early results, Egardt[33] deve]oped a unified framework for the stability analysis of adaptive control algorithms, both in discrete and continuous time. These include some well known model reference schemes, clarke and Gawthrop's self-tuning controller with no control weighting, Astrom's self-tuning regulator and pole placement a.lgorithms. Fgardt's results are for uniform boundedness or $1_{\infty}$ stability 
INTRODUCTION

(see section 1.3 for definition).

Egardt formalised a heuristic argument for stability: If the parameter estimates are such that the closed loop system is unstable, the output increases and the disturbances become relatively insignificant. The estimates should then become more accurate and give a stable closed loop system. The defects of such an argument are well known. Not all the estimates may become accurate and even if they do, perhaps not quickly enough. Egardt's analysis identifies the requirements for eliminating these defects.

The analysis assumes that the number of poles and zeros of the system is accurately known. The integer delay $k$ is also assumed known and the process is minimum phase.

Most of Egardt's analysis is for algorithms which use a stochastic approximation type of estimator. These however are different from those considered in this thesis (cf section 3.8) in that they have a bilinear structure: the first coefficient of $B\left(q^{-1}\right), b_{0}$ and the remaining parameters $\theta$ are estimated separately and appear as a product in the estimated prediction error. Furthermore, the estimator requires an a priori estimate of $b_{0}$, called $\beta_{0}$. This variety of estimator is popular with model reference type algorithms.

with this estimator, it is shown that his generalised adaptive control algorithm will give uniformly bounded control if the a priori estimate, $\beta_{0}$ is chosen to be at least half $b_{0}$ and if the parameter estimates are uniformly bounded. Be also proposes estimator modifications to ensure that the latter condition is satisfied. 
It is important to note that the results of Egardt are valid whether or not disturbances are present and for all values of delay (provided they are known). Some preliminary results were also presented for a least squares type estimator.

Egardt also showed that in the absence of disturbances, his algorithms would converge, in the sense that the output would track a desired model.

A different approach was taken by Gawthrop[38],[40]. Stability is considered in the $I_{2}$ rather than $I_{\infty}$ sense. The algorithm is represented by an exror feedback system, details of which will be presented in section 3.2. It is shown that stability of this error feedback system implies stability of the closed loop system under self-tuning control. The stability of the error feedback system is analysed using the established methods of input-output stability. It is this approach which will be used in this thesis to study the robust stability of the self-tuning controller.

An advantage of Gawthrop's approach is that convergence follows almost txivially from stability. It was shown, using the method of martingales (to be discussed later), that given stability and the positive real condition (Eqn. 21 above), that

$$
\lim _{N \rightarrow \infty} \frac{I}{N} \sum^{N} \bar{e}^{2}(t)=0 \text { w.p. } 1
$$

where $\vec{e}$ is the difference between the prediction error if the parameters are known $[e(t)]$ and the estimated prediction error $\left[\hat{e}_{1}(t)\right]$. Thus this is convergence in the sample mean square sense. 
INTRODUCTION

Egardt's analysis was in part motivated by the stability assumption in Ijung's convergence analysis. Gawthrop's convergence result followed naturally from the stability analysis. For systems without any disturbances, some stability and convergence results were also presented by Goodwin, Ramadge and Caines[44]. Of more interest is a second paper, by the same authors[47]. This presents a 'combined' proof of stability and convergence for systems with stochastic disturbances i.e. those discussed in section 1.1.

The authors consider a minimum variance regulator (both single input-single output and multivariable) and hence a minimum phase assumption is needed. The estimator used is a stochastic approximation type similar to that which is discussed in detail in section 3.8. Note that unlike Egardt's, this estimator is linear in the parameters. Furthermore, this estimator must be modified to handle different delays, $k$, a technique the authors call multiple recursion (cf Chapter 4).

In their proof, Goodwin et al utilise the properties of a class of stochastic processes called positive supermartingales, $z(t)$, which have the property that

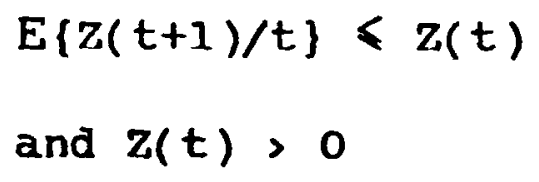

Doob[31] has shown that $z(t)$ can be decomposed into a decreasing sequence and a process called simply a martingale which obeys Eqn. 23 above with a strict equality. For a martingale with a uniformly bounded variance and hence for the associated supermartingale, Doob shows that as $t \rightarrow \infty, z(t)$ converges to a finite constant which is realisation dependent. Solo[78],[79] has also developed a similar convergence theorem for 


\section{INTRODUCTION}

positive supermartingales, which he calls stochastic lyapunov functions. In Goodwin's proof, a stochastic Lyapunov function is defined in terms of the difference between the true and the estimated parameters. Using the above mentioned convergence results, it is shown that

$$
\lim _{N \rightarrow \infty} \frac{1}{s(N)} \sum^{N}[y(t)-w(t-k)-F \xi(t)]^{2}=0 w \cdot p \cdot I
$$

where $s(N)$ is a strictly increasing sequence, the inverse of which forms part of the gain factor in Eqn. 16. Without going into details, it is then possible to show that for minimum phase systems

$$
\lim _{N \rightarrow \infty} \frac{1}{N} \sum^{N}[y(t)-w(t-k)-E \xi(t)]^{2}=0 w \cdot p \cdot I
$$

From these the authors deduced that the stability and convergence results in terms of sample mean square quantities i.e. it is shown that

$$
\begin{aligned}
& \lim _{N \rightarrow \infty} \sup \frac{1}{N} \sum^{N} Y^{2}(t)<\infty \\
& \lim _{N \rightarrow \infty} \sup \frac{1}{N} \sum^{N} u^{2}(t)<\infty \\
& \text { and } \lim _{N \rightarrow \infty} \frac{1}{N} \sum_{E\left\{[Y(t)-w(t-k)]^{2} / \Xi_{t-1}\right\}=\gamma^{2}}^{N}
\end{aligned}
$$

where $\Xi_{t-1}$ represents all data available till time $t-1$ and $\gamma^{2}$ is the minimum possible mean square error achievable with causal feedback.

Besides the results summarised above, much other work has been done on the stability and convergence problem. some of these are for specially modified a]gorithms eg. Cordero and Mayne[23]. others treat only the disturbance free case eg. Goodwin and $\sin [46]$. It is important to note that all the results summarised above are for implicit algorjthms. 
INTRODUCTION

\subsection{MATHF.MATICAT, PRF,T,IMLNARIES}

In this thesis we shall be concerned with signals which are (possibly) infinite sequences of real numbers. Fach gignal may be regarded as a vector of infinite dimension and constitutes an element of a set, known as a linear vector space, in which addition and scalar multiplication is defined. Formal and complete definitions for most of what will be presented in this section can be found in standard books on functional analysis and also in the early papers on input-output stability eg. Zames [91],[92], Sandberg [76].

The concept of magnitude is represented by norms, which are denoted by . and which map each signal or vector to some non-negative value. I.et $\mathbf{x}$ be an element of the linear vector space $i . e$.

$$
x=\left\{\xi_{1}, \xi_{2}, \ldots \ldots, \quad\right\}: \xi \text { are real numbers }
$$

Some common norms which will be used are

$$
\begin{aligned}
& \text { a) } 1_{2} \text { norm: }\|x\|_{2}=\left[\sum_{\infty}^{\infty}\left|\xi_{i}\right|^{2}\right]^{1 / 2} \\
& \text { b) } 1_{\infty} \text { norm: }\|x\|_{\infty}=\sup _{i \geqslant 1}\left|\xi_{i}\right|
\end{aligned}
$$

If these norms exist, the corresponding vector space is known as a normed vector space $\left(I_{2}\right.$ or $\left.I_{\infty}\right)$. To allow for the fact that these norms may not exist, we will use the now familiar device of a truncation. Instead of saying that $x$ is an element of an $1_{2}$ space, we say that $x$ belongs to the extended space, $1_{2 \mathrm{e}}$ if

$$
\|\times\|_{T}=\left[\sum^{T}\left|\xi_{i}\right|^{2}\right]^{1 / 2}<\infty \forall T \text { in set of positive integers. }
$$

Unless otherwise stated, all signals will be assumed to be in $1_{2}$ or $1_{2 e^{*}}$ (without subscript) will refer to $1_{2}$ norms. 


\section{INTRODUCTION}

operator or Mapping will be used synonymously and will refer loosely to the rejationship between an ejement in one space with another element in the same space. A common example is the ARMA model of Chapter $2, L\left(q^{-1}\right)=\frac{B}{A}$. If $A$ has stable roots, $L\left(q^{-1}\right)$ maps an $I_{2}$ sequence of inputs $u$ into an $l_{2}$ sequence of outputs, $y$.

The gain of an operator, $I_{4}: x \rightarrow y$, where $x$ and $y$ belong to $I_{2}$ is defined as the smallest $\gamma$, such that

$$
\|y\|=\|x\| \leqslant \gamma_{1}\|x\|+y_{2} \quad \forall x \text { and arbitrary constant } \gamma_{2} \text {. }
$$

When $\gamma_{2}=0$, it is properly called the induced norm, but for convenience, norm will be used, where there is no possibility of confusion with the $I_{2}$ norm.

An operator I, can also be characterised by conic bounds: constants $c$ and $r$, defined such that

$\|L x-c x\| \operatorname{lax} \quad \forall x$ in the space.

$c$ is known as the centre and $r$ is the radius of the cone.

with $1_{2}$ spaces, there is yet another characterisation for an operator: sector bounds and a related property, passivity. Generalising the common scalar product for finite dimensional vectors, we have the scalar product of two infinite sequences, $x, y$ defined as the real number,

$$
\langle x / y\rangle=\sum^{\infty} x_{i} y_{i}
$$

An operator $I$ is said to be within a sector $(\alpha, \beta)$ if

$$
<\mathrm{Ix}-\alpha \mathrm{x} / \mathrm{Ix}-\beta \mathrm{x}\rangle \leqslant 0
$$

I is passive if it is in the sector $[0, \infty)$. 
INTRODIJTION

In Chapter 4, we will need stronger characterisations of operators. These are the incremental versions of gain, conic bounds and sectors defined respectively from:

$$
\begin{aligned}
& \left\|T_{1}-I_{1} x_{2}<y_{1}\right\| x_{1}-x_{2} \forall x_{1}, x_{2} \in I_{2} \\
& M\left(I x_{1}-T x_{2}\right)-c\left(x_{1}-x_{2}\right)<x_{1}-x_{2} \quad \forall x_{1}, x_{2} \in I_{2} \\
& \left\langle\left(L x_{1}-L x_{2}\right)-\alpha\left(x_{1}-x_{2}\right) /\left(L x_{1}-L x_{2}\right)-\beta\left(x_{1}-x_{2}\right)\right\rangle \leqslant 0 \quad \forall x_{1}, x_{2} \in 1_{2}
\end{aligned}
$$

It can easily be shown that for linear operators, the incremental. gain is the same as the gain and similarly for conic bounds and sectors.

In this thesis, we shall largely be concerned with the stability of a feedback system, of the form of Fjg. I below.

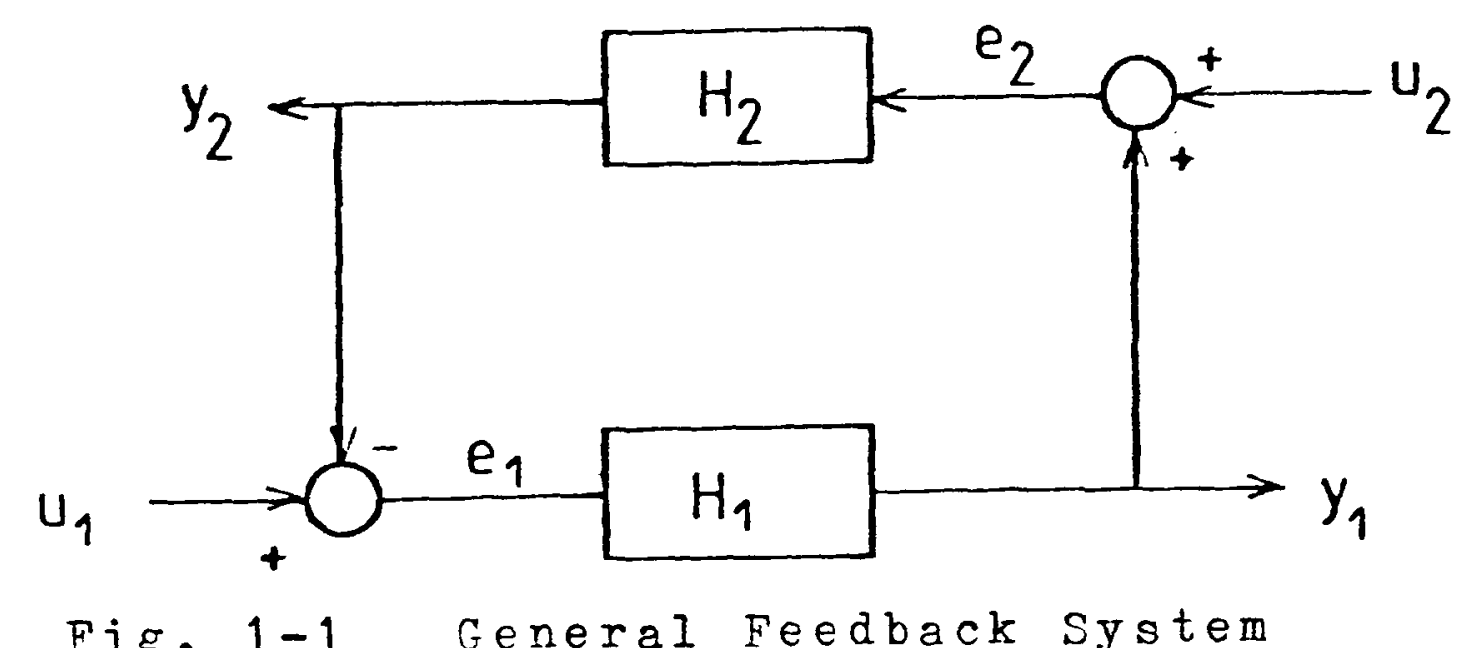

By stability, we shall mean that if $u_{1}$ and $u_{2}$ are 'bounded' sequences (in some sense), then output sequences exist and are also 'bounded'. A common measure of 'boundedness' is if the $1_{2}$ norm exists. Ioosely speaking, signals with $I_{2}$ norms have finite energy. 
TNTRODUCTION

Next we state a version of the small gain theorem which ensures the stability of Fig. 1. Fxcept in Chapter 4 (which justifies it) we assume that solutions exist for the feedback systems derived.

Small Gain Theorem (Desoer and vidyasagar[28])

Consider first that all signals in Fig. 1 are elements of the extended space, $I_{2} e \cdot \mathrm{H}_{1}$ and $\mathrm{H}_{2}$ are operators with gains $\gamma_{1}, \gamma_{2}>0$ such that

$$
\begin{array}{ll}
\mathrm{H}_{1} \mathrm{e}_{1}\left\|_{\mathrm{T}}<\gamma_{1}\right\|_{\mathrm{e}_{1}}+\beta_{1} & : \beta_{1} \text { a constant } \\
\mathrm{H}_{2} \mathrm{e}_{2} \|_{\mathrm{T}} \leqslant \gamma_{2}{\| \mathrm{e}_{2}}_{\mathrm{T}}+\beta_{2} & : \beta_{2} \text { a constant }
\end{array}
$$

If the product of the gains $\gamma_{1} \gamma_{2}<1$, then the feedback system is $I_{2} e$ bounded in the sense that $u_{1}, u_{2} \epsilon I_{2} e$ implies that $e_{1}, e_{2}, y_{1}, y_{2}$ are ejements of ${ }^{1} e$. Furthermore if $u_{1}, u_{2}$ belong to the unextended space, $1_{2}$, then so do $e_{1}, e_{2}, y_{1}$ and $y_{2}$.

\section{Proof}

see Desoer and vidyasagar $[28]$.

\section{Remarks}

It is possible to state equivalent conditions in terms of conic bounds and sector. This will not be done here. Instead appropriate references will be made when these are used.

The small gain theorem is only sufficient and not necessary. As such it is possibly highly conservative. Furthermore it is restricted to feedback systems in which the operators are themselves stable. 
INTRODUCTION

\subsection{OUTT,INF OF THF.STS}

In Chapter 2., the system (or plant) whose stability will be studied when under self-tuning control is modelled as a composition of a nominal linear plant of known structure and plant perturbations which may be linear or nonlinear. The self-tuning controller of clarke-Gawthrop will next be described in some detail and error feedback systems for the perturbed plants under self-tuning control will be formulated. Two types of plant perturbations are considered: the output error case where the plant perturbation is at the output of the nominal plant and the input error case. It will be shown that there is little difference between the two in the linear case. In the nonlinear case, such a structure would fit for example, the case of nonlinearities due to transducers (output) or actuators (input).

The bulk of Chapter 2 will consider the robust stability of the non-adaptive clarke-Gawthrop controller, designed when the parameters of the nominal linear plant are known. Conditions are derived for the stability of the perturbed plant, in terms of the design parameters $P, Q$, $R$ and a predictor filter $C$ defined later. It is also shown that in this case, it is possible to select the design parameters of the Clarke-Gawthrop self tuner to give robustness properties identical to those which would be obtained from the use of two well known pole placement algorithms (Astrom's and Wellstead's).

Chapter 2 concludes with some resultg to show that the design parameters can also be selected to give robust disturbance attenuation. Some examples are used to illustrate the results for both linear and nonlinear perturbations. 
INTRODUCTION

In Chapter 3, we consider stability of the perturbed plant under the control of an adaptive clarke-Gawthrop self tuner designed for the nominal plant. The main difference from the nonadaptive case is the presence of a nonlinear, time-varying operator which represents the estimator. The chapter thus begins with the input-output properties of this estimator block. These are then used to review and extend the stability results of Gawthrop[40] for the unperturbed plant.

Robust stability results for the adaptive controller, when the delay is minimal follow in the next section. Both linear and nonlinear perturbations are considered, though in the latter only for the case of $Q=0$ (no control weighting). As these results are possibly conservative, some instability results are also presented in the next section.

Thus far in Chapter 3, only implicit algorithms have been studied. In the next section, the problem of studying stability for explicit algorithms is explained. An important special case is considered, which suggests that explicit self tuners may not be as robustly stable for nomminimum phase systems as has often been claimed if there are plant perturbations.

For the basic Clarke-Gawthrop self tuner, the robustness properties depend on a scalar sequence, $\sigma(t)$, which has to be assumed small. Section 3.7 studies this sequence and reveals some conditions under which it is indeed small. Alternatively, a different estimator may be used. This has a scalar gain adjustment factor and is similar to that used by Goodwin [ 44]. 
INTRODUCTION

In Chapter 4, the effect on robust stability of some practical modifications is studied. First the minimal delay assumption of Chaptex 3 is removed. Next the case of $Q \neq 0$ is considered. Chapter 4 concludes with an analysis of robust stability when varjous methods for offset elimination are included.

In Chapter 5, simulation examples are used to illustrate the results of the thesis. These examples also serve to demonstrate some of the tradeoffs which have to be made in the design of the self-tuning controllex.

The conclusions of this thesis are stated in Chapter 6 . 


\section{CHAPTF.R 2}

ROBUSTNESS OF THE NONADAPTIVE CONTROT,T.F.R

\subsection{INTRODUCTION}

This chapter is mainly devoted to the robustness of the non-adaptive design method that is the basis of the clarke-Gawthrop self-tuning controller (NA C-G STC). One motivation for studying the non-adaptive version first is that being a certainty equivalent control law, it appears unlikely that the adaptive version will be robust unless the non-adaptive version is. Furthermore analysis in the non-adaptive case is simpler and will be used to provide guidelines for the choice of design parameters.

Chapter 2 begins with a review of some classical stability results which will be used later. In section 2.3, the error feedback system for the clarke-Gawthrop self-tuning controller is derived. The rest of the chapter deals with the non-adaptive version.

\subsection{RFVIEW OF STANDARD STABIIITTY RESUTTS}

Throughout this chapter, all systems will be assumed to be discrete-time, single input single output, causal and time-invariant unless otherwise stated. 
ROBUSTTNESS OF THE NONADAPTIVF, CONTROIITER

For linear systems within this class, represented by ARMA type models, the Nyquist theorem is a simple and graphical means of testing stability.

\section{Theorem I (Nyquist)}

In the feedback system of Fig. 2-1 below, necessary and sufficient conditions for $l_{p}$ stability are:

J. $\quad 1+\mathrm{H}_{1}(\mathrm{O}) \mathrm{R}_{2}(0) \neq 0$

2. the Nyquist locus of $\mathrm{B}_{1}\left(\mathrm{q}^{-1}\right) \mathrm{B}_{2}\left(\mathrm{q}^{-1}\right)$ does not pass through $(-1,0)$

3. the Nyquist locus of $\mathrm{H}_{1}\left(\mathrm{q}^{-1}\right) \mathrm{H}_{2}\left(\mathrm{q}^{-1}\right)$ encircles the $-1+j 0$ point $\mathrm{m}_{\mathrm{p}}$ times in the anticlockwise direction, where $m_{p}$ is the number of poles of $\mathrm{B}_{1}\left(\mathrm{q}^{-1}\right) \mathrm{B}_{2}\left(\mathrm{q}^{-1}\right)$ in the unstable region

4. ${ }_{2}\left(q^{-1}\right)$ does not have zeroes which cancel the poles of $B_{1}\left(q^{-1}\right)$

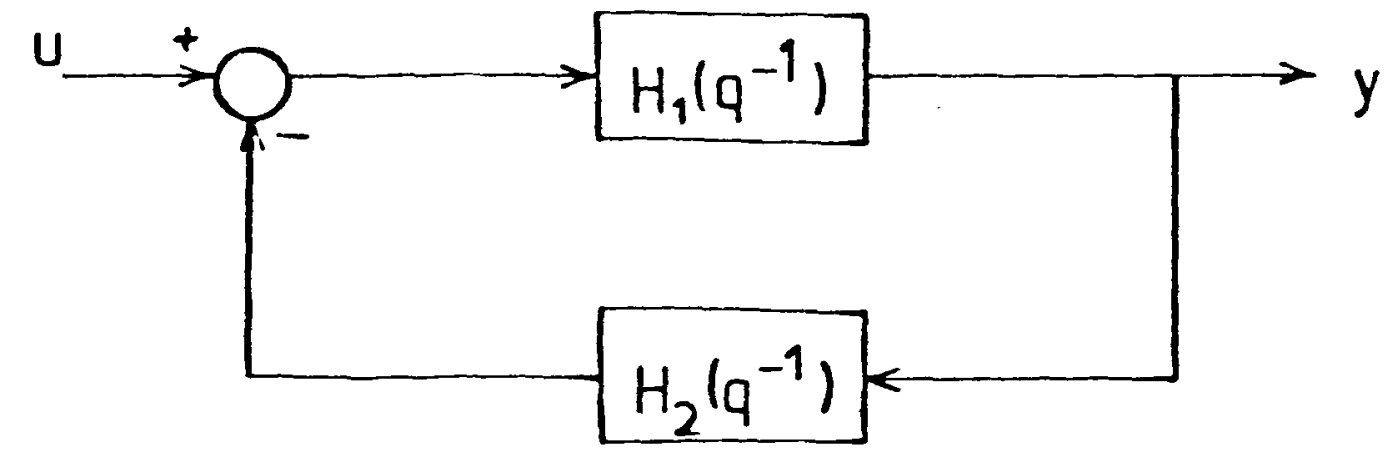

Fig. 2-1 Typical Feedback system

The Nyquist locus of $B\left(\mathrm{q}^{-1}\right)$ is simply the magnitude and phase locus for $B\left(e^{j \theta}\right)$ as $\theta$ varies from 0 to $2 \pi$. If $B\left(q^{-1}\right)$ has poles on the unit circle, then the domain of $\mathrm{B}\left(\mathrm{e}^{\mathrm{j \theta}}\right)$ is suitably indented. 
ROBUSTNF.SS OF THF. NONADAPTIVE CONTROIITIFR

\section{Proof}

See Desoer and Vidyasagar[28]. The proof is developed in terms of the 2 transforms. It is well known however that the $z$ transform version of $\mathrm{A}\left(\mathrm{q}^{-1}\right)$ is simply $\mathrm{B}\left(\mathrm{z}^{-1}\right)$ where $z$ replaces $\mathrm{q}$.

The Nyquist locus yields not only the stability theorem above, but robust stability measures in the well known gain and phase margins. The gain margin is defined as the additional gain required at a phase angle of $-180^{\circ}$ to cause instability while the phase margin is the additional phase lag necessary for instability when the magnitude of $\mathrm{H}_{1}\left(q^{-1}\right) \mathrm{B}_{2}\left(q^{-1}\right)=1$. Together the gain and phase margins may be interpreted as providing for unmodelled gains or dynamics. In classical compensator design for continuous time systems, Horowitz[50] has long developed this approach for designing controllers which are insensitive to plant perturbations.

When either $\mathrm{H}_{1}$ or $\mathrm{H}_{2}$ in Fig. $2-1$ is nonlinear, the most general stability test that can be used is the small gain theorem. [Chapter 1], willems[88], Desoer[28] etc. This is a generally conservative test. The Popov and circle type stability criterion are specialisations of the small gain theorem, which have a graphical frequency domain interpretation and are thus easier to use. They require that the Nyquist locus (or modified Nyquist locus in the case of the Popov criterion) avoid some critical region, the size of which depends on the nonlinearity. 
ROBUSTNF.SS OF THF. NONADAPTTVF. CONTROT,T.F.R

A discrete-time version of the circle criterion will be stated in Section 2.4.1. Here we note that most of the nonlinear stability criterion can be interpreted as imposing sector type bounds on the nonlinearity, given the Nyquist locus of the linear subsystem. This is a robust stability result in that any nonlinearity or time-varying linear operator meeting the sector requjrement is allowed. The sector bound can a]so be interpreted as a bound on possible system perturbations. It is reasonabje to suppose that the further away the Nyquist Jocus is from the critjcal region, the more robust the closed joop system is to perturbations. Recently Jiejtch,R.R.[6]] has defined a quantitative measure for a margin of boundedness, $\delta$ which requires that the Nyquist Jocus is not in the sector

$$
\frac{2(1-\delta)}{-2 \alpha+\delta(\alpha+\beta)}, \frac{2(1-\delta)}{-2 \beta+\delta(\alpha+\beta)}
$$

where $(\alpha, \beta)$ is the sector bound of the nonlinearity. Teitch also shows that $\delta$ can be viewed as a generalisation of gain and phase margins in the linear case.

The subject of robust stability is connected in many ways with sensitivity theory. (see eg. Cruz et al[24]). It has been well known for a long time that for linear systems, the return difference equation, $\left[1+\mathrm{H}_{1} \mathrm{R}_{2}\right]$ in Fig. 2-1, should be large over the largest possible frequency range to reduce sensitivity of the closed loop system to plant parameter variations. In section 2.5, we will show that a similar requirement is necessary for robust disturbance attenuation, in both linear systems and a restricted class of nonlinear systems. 


\section{2. MODF.T, OF PFRTIJRBF.D SYSTF.M}

In this thesig, the system or plant, as seen by the controller is assumed to have the structure shown in Fig. 2-2. below.

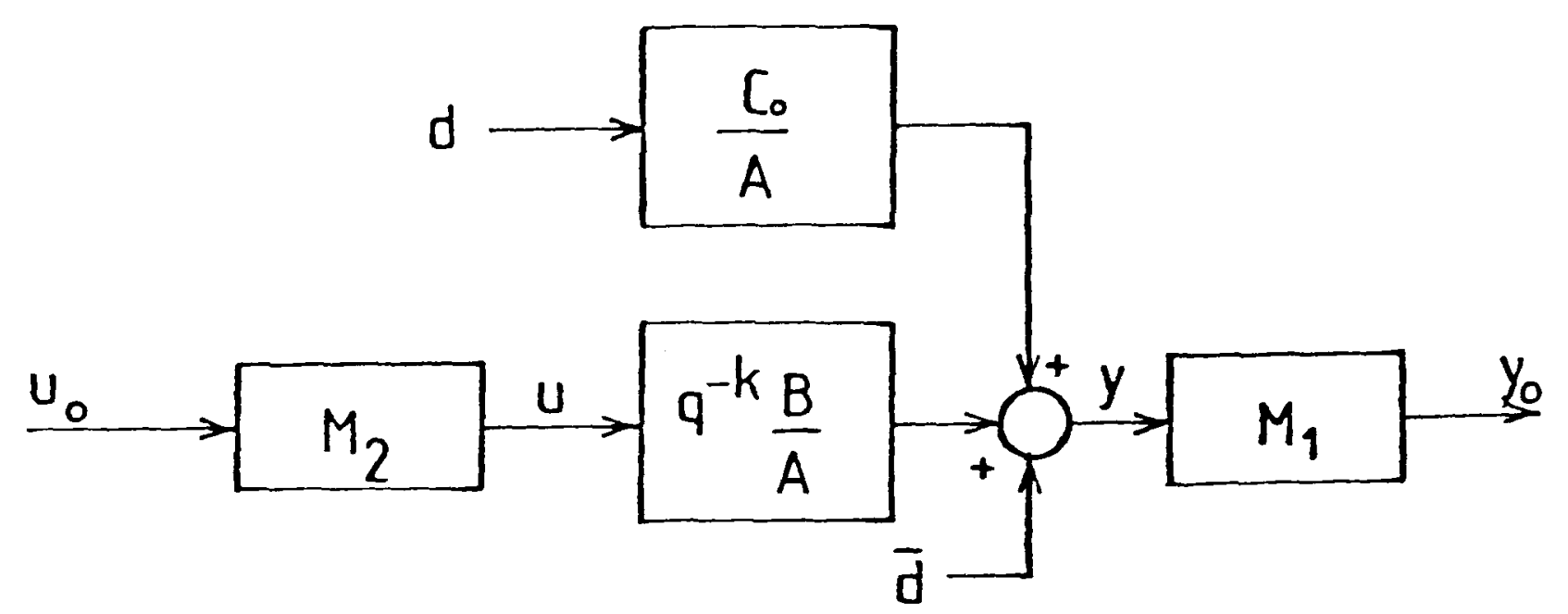

Fig. 2-2 Structure of System Model

If $M_{1}=M_{2}=1$, then the system of Fi.g. 2-2. reduces to that commonly assumed for the design of self-tuning type algorithms $[2.0],[9],[85]$ etc. A, B, C are polynomials in the backward shift operator, $q^{-1}$.

In the sejf-tuning jjterature, $d(t)$ is commonly taken to be a zero mean uncorrejated random varjable. Here we shall only requjre that $d(t)$ be zero mean and that jt be a sujtably bounded discrete sequence. To account for non-zero mean disturbances, we jncjude the constant $\bar{d}$. In most of what follows, $\bar{d}$ will be taken to be zero unless otherwise stated.

sections 2.2.1, 2.2.2. show that the system of Fig. 2-7. is sufficiently general to include a large class of systems. 


\section{2..1 Higher order systems}

Assume that the system can be described by a higher order ARMA model

$$
A_{0}\left(q^{-1}\right) y_{0}(t)=q^{-k} B_{0}\left(q^{-1}\right) u(t)+C_{0}(t)
$$

where

$$
\begin{aligned}
& \operatorname{deg}\left(A_{0}\right\} \geqslant \operatorname{deg}\{A\} \\
& \operatorname{deg}\left\{B_{0}\right\} \geqslant \operatorname{deg}\{B\}
\end{aligned}
$$

It is easily shown that if

$$
M_{2}=1, \quad M_{1}=\frac{A B}{B A} 0 \text { and } d(t)=\frac{B}{B_{0}} d_{0}(t)
$$

then the system of Fqn. 1 fits into the structure of Fig. $2-2$. Thus $q^{-k B} \frac{B}{A}$ would represent modeljed dynamics and $M_{1}$ would be a linear operator that contains unmodelled dynamics. Alternatively, let

$$
M_{2}=\frac{A B}{B A}{ }_{0}^{D} \quad \text { and } \quad M_{1}=1
$$

Since scalar linear operators commute, the system of Eqn. I also fits the structure of Fig. $2-2$ if

$$
d(t)=\frac{A}{A_{0}} d_{0}(t)
$$

later it will be necessary to assume that $d(t)$ is a bounded discrete sequence (i.e. $\epsilon 1_{2}$ ). If $\alpha_{0}(t) \epsilon 1_{2}$ and if either $A_{0}$ or $B_{0}$ is stable, $d(t)$ will correspondingly be an element of $1_{2}$. The two alternatives do not make any difference to the subsequent analysis. If however, both $A_{0}$ and $B_{0}$ are unstable, it will have to be assumed that $d_{0}(t)$ can be written as

$$
d_{0}(t)=M_{u}\left(q^{-1}\right) d^{1}(t)
$$

where $a^{1}(t) \in I_{2}$ and $M_{u}\left(q^{-1}\right)$ contains the unstable roots of $A_{0}$ or $B_{0}$. 
ROBUSTNF.SS OF THF, NONADAPTIVF, CONTROT,T.F.R

\subsubsection{Nonlinearities}

If $d(t)=0$, it is easily seen that Fig. 2-2. reduces to a standard cascade connection of linear dynamics and nonlinear elements. The simplest physical justification for the structure would be when $M_{1}$ represents nonlinear transducers and $M_{2}$ represents actuators on a linear dynamical system. In the subsequent analysis, ejther $M_{1}=1$ or $M_{2}=1$ i.e. we only consjder a system with an input nonlinearity or a system with an output nonlinearity.

Such a cascaded system is commonly assumed in the identification literature [13],[12]. For instance, a commonly used model for a nonlinear system is the Hammerstein model.

$$
A\left(q^{-1}\right) Y(t)=a_{1} B\left(q^{-1}\right) u(t-k)+a_{2} B\left(q^{-1}\right) u^{2}(t-k)+\ldots
$$

Eqn. 2 can be expressed in bjock diagram form as an input static nonlinearity followed by linear dynamics. Thus

$$
\mathbf{m}\{u\}=a_{1} u+a_{2} u^{2}+a_{3} u^{3}+\ldots
$$

A simplified form of the wiener series for nonlinear systems can be similarly represented as linear dynamics followed by output static nonlinearities[14]. Bere the output static nonlinearities must be an onto and one-to-one function.

The kernel functions of another commonly used representation, Volterra series, are interrelated to those of the wiener series[60] and can hence also be regarded as output nonlinearity following linear dynamics. 
ROBUSTNA.SS OF THF, NONADAPTIVF. CONTROIJIF.R

\subsubsection{Other Repregentations}

In the literature on sensitivity theory, plant perturbations are usually regarded as additive (Fig. 2-3) or multiplicative (Fig. 2-4)

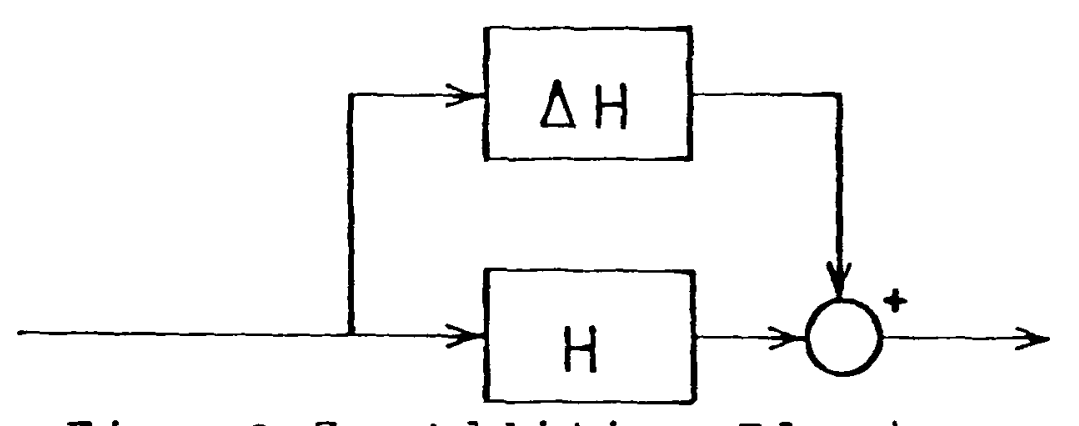

Fig. 2-3 Additive Plant Perturbation

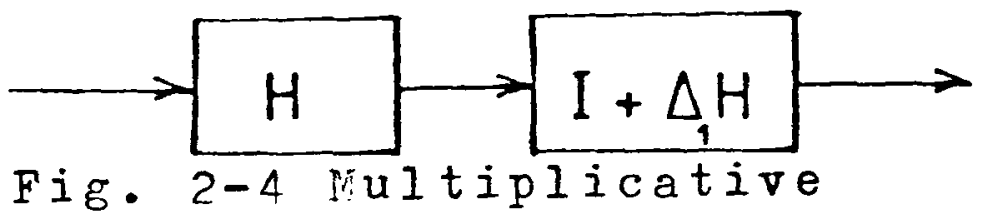

plant perturbation

Here $\mathrm{H}$ is the nominal plant, corresponding to $\mathrm{q}^{-\mathrm{k}} \frac{\mathrm{B}}{\mathrm{A}}$ in Fig. $2-2$.

Comparing Figs. 2, 3, 4 it is easy to show that, if disturbances are considered to be at the output, we have

Lema 1

$$
\begin{aligned}
& \Delta B=\left(\Delta_{1} B\right) B \\
& M_{1}=I+\Delta_{1} B \\
& H_{2} B^{-1}=M_{1} \\
& \Delta B=B\left(M_{2}-1\right) \\
& \Delta B=\left(M_{1}-1\right) H
\end{aligned}
$$

\section{Proof}

In Appendix A.I

$\square$ 
ROBUSTTN.SS OF THF. NONADAPTTVF. CONTROT,TIFR

T.emma 1 will. be useful for comparing results.

The structure of perturbation assumed is thus largely a matter of convenjence as Lemma 1 shows that they are equivalent. Additive perturbation is preferred by, eg. Krejselmeir[53], Anderson[4].

Prom Fqn. $7, \mathrm{M}_{2}-1=\mathrm{B}^{-1}[\Delta \mathrm{H}]=\mathrm{B}^{-1}\left[\Delta_{1} \mathrm{H}\right] \mathrm{H}$

and from Eqn. $8, M_{1}-1=[\Delta \mathrm{B}] \mathrm{H}^{-1}=\left[\Delta_{1} \mathrm{H}\right]$

$\left[\mathbf{M}_{\mathbf{i}}\right.$ - 1] may then be regarded as "percentage perturbations" from nominal plant and will be extensively used in the subsequent analysis. Also used is $\left[1-M_{1}^{-1}\right]$ which translates into $\Delta B[H+\Delta E]^{-1}$ and may hence be regarded as "percentage perturbation" from total plant $B+\Delta H$.

$$
\text { Similarly, }\left[1-M_{2}^{-1}\right]=[H+\Delta B]^{-1} \Delta B
$$

\subsection{ERROR PEEDRACK SYSTEM}

In this section, the self-tuning algorithm is described. Error signals are defined which will be used in the rest of the thesis to set up feedback systems for the study of robust stability.

\subsubsection{The Self-tuning Algorithm}

The basic self-tuning controllex has been briefly described in Chapter 1, where it is applied to a linear time-invariant plant of known order and delay. In this section, the equations necessary for robustness analysis are stated. 
In the self-tuning controller of clarke and Gawthrop[19],[20], an auxiliary process $\Phi(t)$ is defined where

$$
P(t)=P\left(q^{-1}\right) Y_{0}(t)
$$

and $P\left(q^{-1}\right)$ is a polynomial in the backward shift operator $q^{-1}$. If in the plant structure defined by Fig. 2-2,

$$
M_{1}=M_{2}=1
$$

$a(t)=\xi(t)=a$ white noise process, then the steady state, least squares prediction of the process $\Phi(t)$ is given by

$$
\Phi^{\star}(t+k / t)=\frac{P}{C} Y(t)+\frac{B E}{C} u(t)
$$

where $F, E$ are polynomials in $q^{-1}$, given by the identity [20]

$$
\frac{C P}{A}=E+q^{-k F}
$$

Here, a unique solution is obtained by fixing

$$
\text { degree }\left\{F_{0}\right\}=k-1
$$

In this analysis, we wish to consider a general d( $t$ ) subject only to a boundedness assumption to be defined later.

Eqns. 13 to 15 together can be interpreted as a non-optimal predictor for $\Phi(t)$. The poles of the predictor are thus the roots of $c\left(q^{-1}\right)$.

To realise the predictor, we use an algorithm analogous to recursive least squares.

Let $x(t)$ be the filtered vector of past and present inputs and outputs.

$$
x^{T}(t)=\frac{1}{c}\left\{u(t), u(t-1), \ldots, u\left(t-n_{G}\right), y(t), y(t-1), \ldots, y\left(t-n_{F}\right)\right\}
$$

where

$$
\begin{aligned}
& n_{G} \text { is the assumed order of } G\left(q^{-1}\right)=F\left(q^{-1}\right) B\left(q^{-1}\right) \\
& n_{F} \text { is the assumed order of } F\left(q^{-1}\right)
\end{aligned}
$$


Iet $\theta(t)$ be the vector of coefficients

$$
\theta^{T}(t)=\left\{g_{0}, g_{1}, \ldots, g_{n_{G} ; f_{0}}, f_{1}, \ldots, f_{n_{F}}\right\}
$$

$g_{i}, f_{i}$ are the coefficients of $G\left(q^{-1}\right), F\left({ }^{-1}\right)$ respectively

The predictor of Eqn. 13 can be written as

$$
\Phi^{*}(t+k / t)=x^{T}(t) \theta(t)
$$

The error between $\Phi(t)$ and its predictor, $\Phi^{\star}$ is defined as

$$
e=\Phi-\Phi^{*}=E a(t) \quad \text { (by substituting Fqns. 11, 14\} }
$$

If the assumptions of Eqn. 12 are true, $e(t)$ is a moving average process of degree $k-1$.

If the assumptions of Eqn. 12 are not true, the components of $x$ are not accessible. We define the filtered data vector $x_{0}{ }^{T}(t)$, of accessible varjables as

$$
x_{0}^{T}(t) \equiv \frac{1}{c}\left\{u_{0}(t), u_{0}(t-1), \ldots ; y_{0}(t), y_{0}(t-1) \ldots\right\}
$$

The self-tuning algorithm may be written as

$$
\begin{aligned}
& \hat{\Phi}_{1}(t) \leqq x_{0}{ }^{T}(t-k) \hat{\theta}(t-1) \\
& \hat{e}_{1}(t) \leqq P\left(q^{-1}\right) y_{0}(t)-\hat{\Phi}_{1}(t)=\Phi(t)-\hat{\Phi}_{1}(t)
\end{aligned}
$$

Estimated parameter vector:

$$
\begin{aligned}
& \hat{\theta}(t)=\hat{\theta}(t-1)+s^{-1}(t-k) x_{0}(t-k) \hat{e}_{1}(t) \\
& s(t)=\beta S(t-1)+x_{0}(t) x_{0}{ }^{T}(t) \\
& s(0)=s_{0}>0 ; 0<\beta \leqslant 1
\end{aligned}
$$

The scalar $\beta$ is the exponential forgetting factor. $\hat{\Phi}_{1}$ is an estimate of $\Phi^{*}$ and $\hat{e}_{1}(t)$ an estimate of $e(t)$.

$s(t)$ is a matrix referred to hereafter as the estimator gain matrix. In practice, $S$ is not realised as in Eqn. 24, but via numerically stable techniques like the $\mathrm{UDO}^{\mathrm{T}} \operatorname{method}[81]$ or the square root method of Peterka[ 73]. 
The algorithm above becomes a controller by defining:

$$
u_{0}(t)=\frac{1}{Q}\left[R w(t)-\hat{\Phi}_{k}(t+k / t)\right]
$$

where $R\left(q^{-1}\right), Q\left(q^{-1}\right)$ are polynomial.s in $q^{-1}, w(t)$ is the desired setpoint and $\hat{\Phi}_{k}(t+k / t)$ i.s the $k$ step ahead predictor for $\Phi(t+k)$.

$$
\hat{\Phi}_{k}(t+k / t) \equiv x_{0}^{T}(t) \hat{\theta}(t)
$$

Note that the control law Fqn. 26 uses the $k$ step ahead predictor while the estimator, Eqn. 23, used the one step ahead prediction error. In Section 4.1 a relationship will be derived between the two. It suffices here to note that the $i t h$. step predictor for $\Phi(i<k)$ cannot be used in the control law because $u_{0}(t)$ will be noncausal and hence not reajisable. This restriction does not apply to estimation and prediction problems.

From the equations above, it is clear that the design parameters which can be chosen by the designer are

1. the $P, Q, R$ and $C$ polynomials

2. system order

3. assumed system delay $\mathrm{k}$

In subsequent sections, some guidelines for the choice of the item 1 parameters to ensure robustness will be derived.

The assumed delay will be taken to be the actual delay unless otherwise stated. The choice of system order will be assumed to be determined by a prior knowledge of the system and by computational capabilities. 
ROBUSTNF.SS OF THF. NONADAPTIVF. CONTROTITER

\subsubsection{Frror Fquations}

In the analysis of adaptive systems, it is now standard practice to formulate the problem as a feedback system of error quantities. In Iandau's work[58] on MRAS controllers, the adaptive control system is reduced to an error feedback system consisting of time-invariant linear dynamics in the forward path and a nonlinear time-varying operator in the feedback path. By imposing a passivity type condition [58] on the feedback block and positive-real conditions on the forward block, the control Jaw is designed to ensure that the closed loop system is stable.

Narendra and I,in[62],[70], have also derived a series of exror models for some continuous and discrete time identification and control algorithms of the model reference type. In their work Lyapunov type techniques are used to derive stable control. laws.

In Gawthrop[38], [40] an error feedback model is formulated to study the stability of linear systems controlled by a full order self-tuning controller. The emphasis in this formulation is on the use of standard results for input-output stability.

The following error quantities are now defined (with reference to Fig. 2-2)

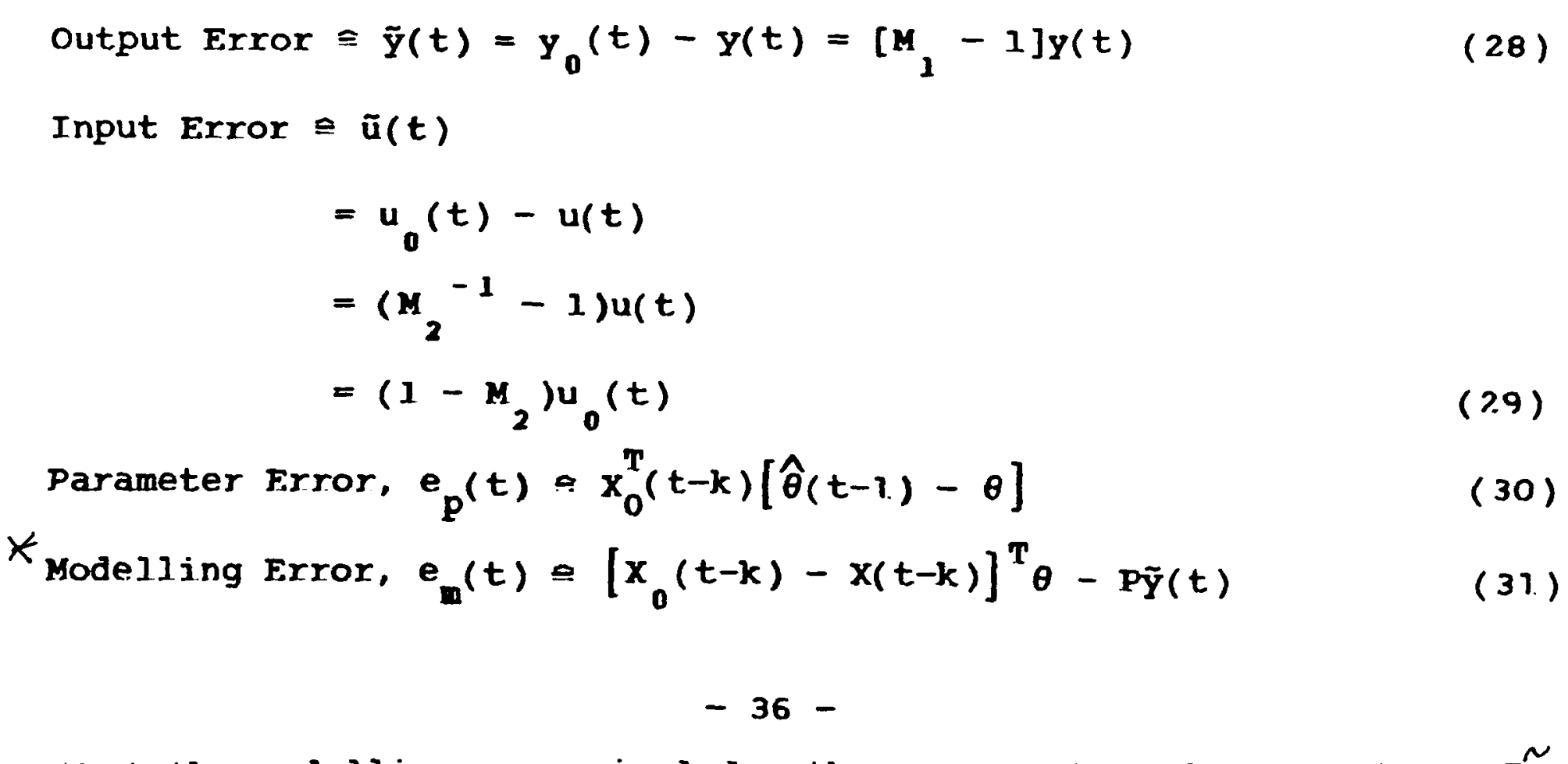

* Note that the modelling error includes the apparently redundant term Py for convenience in deriving the exror feedback system. 
ROBUSTNF.SS OP THF, NONADAPTIVF, CONTROT.T.F.R

From F.qns. 14, 16, 19 of the previous section

$$
e_{m}(t)=-\frac{A E}{C} g(t)+q-\frac{k B E}{C} \mathbf{a}(t)
$$

The input to the estimator update, Eqn. 2.3 is $\hat{e}_{1}(t)$, the prediction error, which from Eqns. 28 to 31 above is

$$
\begin{aligned}
\hat{e}_{1}(t) & =P_{0}(t)-\hat{\Phi}_{1}(t) \\
& =e(t)-e_{m}(t)-e_{p}(t)
\end{aligned}
$$

In Appendix A.2, the following relations will be established.

$$
\begin{aligned}
& y(t)=\frac{B}{P B+Q A}\left[\hat{e}_{1}(t)-P Y(t)-Q u(t-k)+e_{1}(t)\right] \\
& e_{1} \cdot(t)=\hat{e}_{k}(t)-\hat{e}_{1}(t)+R w(t-k)+\frac{C Q}{B} d(t) \\
& u(t-k)=\frac{A}{P B+Q A}\left[\hat{e}_{1}(t)-P Y(t)-Q \tilde{u}(t-k)+e_{2}(t)\right] \\
& e_{2}^{\prime}(t)=\hat{e}_{k}(t)-\hat{e}_{1}(t)+R w(t-k)-\frac{C P}{A} d(t)
\end{aligned}
$$

\section{Assumption 1}

In the subsequent analysis, it will be assumed that either $a=0$ or $8=0$. As shown in section 2.2., there is no loss of generality in the linear case. In the nonlinear case, the assumption means that we only consider an input nonlinearity or an output nonlinearity.

\section{Assumption 2}

$P B+Q A$ is stable

$P$ and $Q$ are design polynomials and hence known. 
ROBUSTNF,SS OF THF, NONADAPTIVF, CONTROT.J.F.R

In the non-adaptive case, $P$ and $Q$ are chosen with $A, B$ being the known nominal plant parameters. Hence Assumption 2 then is really a condition for stability of the nominal design.

In the adaptive case, we will show that for stabilty, there rast exist A, B such that Eqn. 38 is true.

\subsection{ROBUST STABIIITY: THE NON-ADAPTIVE CASE}

In this section, the robust stability properties of the design method for known systems that is the basis for the self-tuning algorithm is examined. We assume that nominal plant parameters are given i.e. A, B polynomials together with the magnitude of possible plant perturbations. The controller parameters are then obtained by solving the identity of Eqn. 14. (ignoring $\mathrm{M}_{2}$ or $\mathrm{M}_{2}$ )

The problem posed is then: How do we select the design parameters to ensure stability in the presence of the plant perturbations?

In this section, as no parameter estimation is used, the parameter error,

$$
e_{p}(t)=0
$$

Hence from the relationship between the error quantities, Eqn. 33,

$$
\hat{e}_{1}(t)=e(t)-e_{m}(t)
$$

The two cases of $\mathbf{a}=0$ and $\bar{y}=0$ are now considered in turn. 
ROBUSTNESS OF THE. NONADAPTIVE, CONTROT,T.F.R

2.4.1 Output Error

Assume that $a=0$

(41)

$$
\begin{aligned}
9= & {\left[M_{1}-1\right] Y \quad \text { (from Eqn. 28) } } \\
= & {\left[M_{1}-I\right] \frac{B}{P B+Q A}\left[\hat{e}_{1}(t)-P g(t)+e_{1} \cdot(t)\right] } \\
& \quad \text { from Eq. } 34 \\
= & {\left[M_{1}-1\right] \frac{B}{P B+Q A}\left[e(t)+e_{1}(t)-\left(P^{-F \cdot A}\right) Y(t)\right] }
\end{aligned}
$$

: from F.qn. 40 and Eqn. 32

substituting the 'self-tuning identity', En. 14, we get

$$
9=\left[M_{1}-1\right] \frac{B}{P B+Q A}\left[e(t)+e_{1}{ }^{\prime}(t)-q^{-K F} \frac{F}{C}\right]
$$

Eq. 44 describes a negative feedback system which is shown in Fig. 2-5 below. Note that in the nonadaptive case,

$$
\begin{aligned}
\hat{e}_{k}(t) & =\Phi(t)-x_{0}{ }^{T}(t-k) \hat{\theta}(t-k) \\
& =\Phi(t)-x_{0}{ }^{T}(t-k) \hat{\theta}(t-1) \\
& =\hat{e}_{1}(t) \\
& =\Phi(t)-x_{0}{ }^{T}(t-k) \theta_{\text {nominal }}
\end{aligned}
$$

Hence $e_{1}(t)=R w(t-k)+\frac{Q C}{B} d(t)$

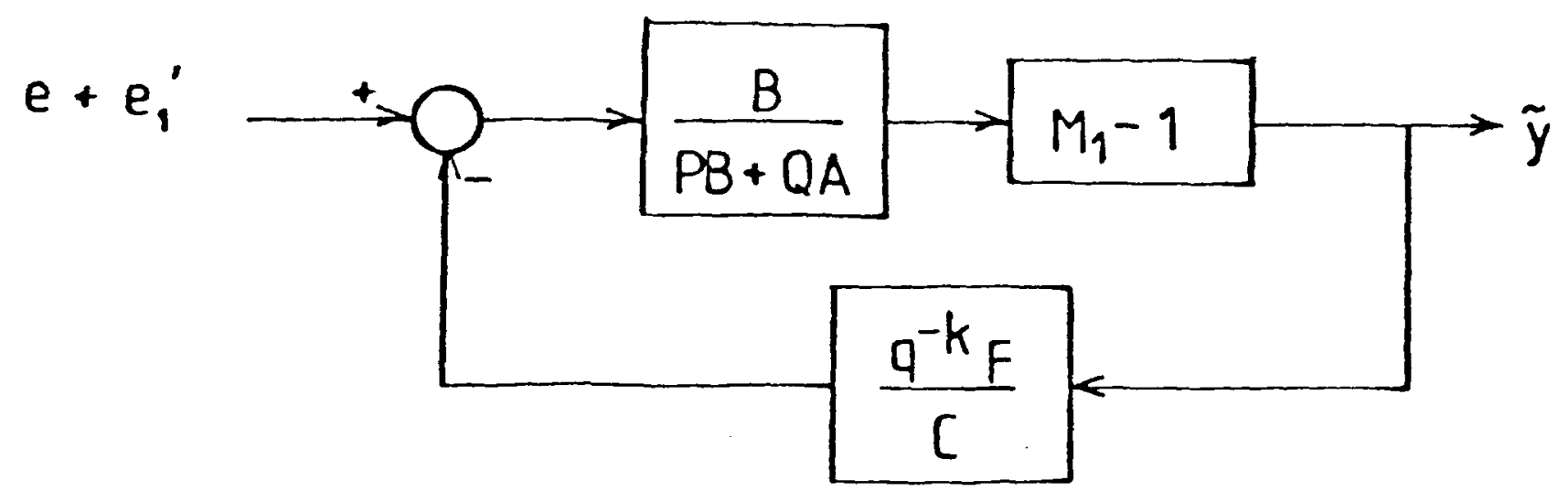

Fig. 2-5 Feedback System for output Error Case

$-39-$ 
ROBUSTNF.SS OF THF, NONADAPTIVF, CONTROIIIFR

\section{Theorem 1}

stability of the feedback system of Fig. 2-5 is guaranteed by any one of the following sets of conditions.

a) $\sup _{|q|=1}\left|\frac{q^{-k} F B}{C(P B+Q A)}\right| \leqslant\left[\operatorname{gain}\left\{M_{1}-1\right\}\right]^{-1}$

b) If $M_{1}$ is linear, $A$ and $A_{0}$ are stable, and $\left|\frac{B_{0}}{A_{0}}-\frac{B}{A}\right| \leqslant\left|\frac{C(P B+Q A)}{q^{-k F A}}\right| \quad v|q|=1$

c) $M_{1}$ is linear and $\left(\frac{B_{0}}{A_{0}}-\frac{B}{A}\right) \frac{q^{-k} F A}{C(P B+Q A)}$ obeys the usual Nyquist stability criterion

Proof

Sets (a), (b) follows from the small gain theorem (Chapter 1) and a well-known result for the gain of an $1_{2}$ mapping in the frequency domain.

Set (C) is the standard Nyquist condition in the open loop transfer. functions.

口

Remark 1

(a) and (b) are only sufficient conditions. This follows from the use of the small gain theorem. (c) is both necessary and sufficient.

\section{Remark ?}


ROBUSTNF.SS OF THF. NONADAPTTVF, CONTROI.T.FR

Set (b) has previously been derived in Astrom[7] for a design method based on the assignment of poles and zeros. In Astrom and wittenmark[11], it is shown that the pole-zero assignment method reduces to the design method used here if $Q=0$ and $B$ has all roots inside the unit circle.

Sets (a) and (b) can be viewed as a bound on the permissible plant perturbation (not necessarily small), which is easily evaluated in the non-adaptive case with known nominal plant. Astrom[7] offers some interesting interpretations of set (b) if $Q=0$.

\section{Remark 3}

Recently Zames[95] has shown that in linear systems a control law may be decomposed into two stages:

a) filtering of plant uncertainty

and

b) design of a control law for the nominal plant. It is easy to see that Fig. 2-5 can also be decomposed in a similar fashion; see Fig. 2-6.

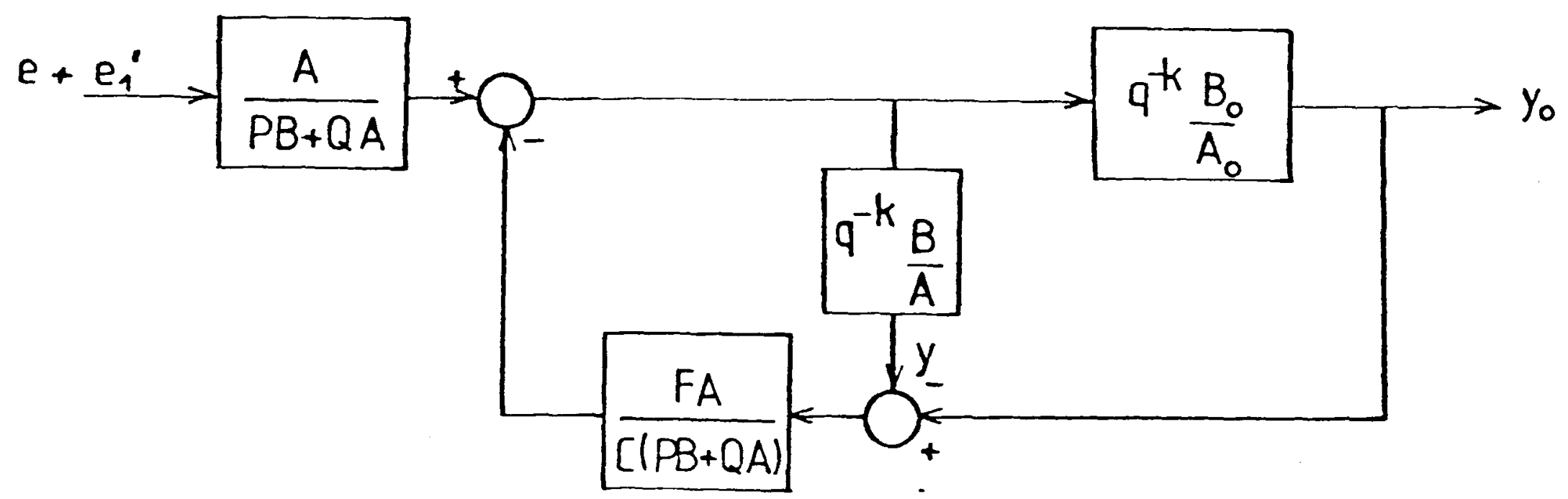

Fig. 2-6 Decomposition of Feedback System for output Error Case 
ROBUSTIF.SS OF THF. NONADAPTIVF. CONTROT,T.F.R

$\frac{F A}{C(P B+Q A)}=\dot{Q}$ can then be regarded as a filter on plant uncertainty, while $\frac{A}{\mathrm{~PB}+\mathrm{QA}}=\dot{\mathrm{R}}$ is a precompensator

Zames[95] also shows that for stability, set (b) is sufficient. Note that this decompositjon allows us to view this control law synthesi.s method in the spirit of Horowitr[50] i.e. design $\ddot{Q}$ for stability in the face of plant uncertainty and then design precompensator $\vec{R}$ to meet other design criteria.

In this context, note also that the input to the system of Fig. 2-6 contains both desired setpoint and disturbance terms. Hence it will not always be possible to meet simultaneously tracking and disturbance attenuation criterion. It is thus clear that $R$, the setpoint filter allows an extra'degree of freedom' in the design. Robust disturbance attenuation and tracking will be discussed in a later section.

\section{Remark 4}

I.et gain $\left\{M_{1}-1\right\}=\delta$.

An obvious graphical interpretation for condition (a), Theorem $I$ is that we require the Nyquist plot of $\frac{\bar{q}^{k} F B}{C(P R+Q A)}$ to lie within a circle of radius $\frac{I}{6}$, centre $(0,0)$ in the complex plane.

\section{Isemma 1}

$$
\text { Assume that } \sup _{|q|=1}\left|\frac{q^{-k} F B}{C(P B+Q A)}\right|=\frac{1}{\delta}
$$

If condjtion (a) of Theorem 1 is met, then the nominal closed loop feedback system has at least a gain margin of $(5+1)$ and a phase margin of 
ROBUSTNFF.SS OF THF. NONADAPTIVF. CONTROT,T.F.R

$$
\begin{aligned}
& \cos ^{-1}\left(1-\frac{1}{2} \delta^{2}\right) \text { iff } 0^{2}<2 \\
& 180^{\circ}-\cos ^{-1}\left(\frac{1}{2} 0^{2}-1\right) \text { if } 4>\delta^{2}>2
\end{aligned}
$$

Proof

In Appendix A.3

Note that the converse does not follow j.e. a certain gain and phase margin of the nominal plant does not necessarjly jmply robust stabijity to perturbations.

\section{Remark 5}

If $M_{2}-$ I is a sector bounded memoryless operator, it is simple to extend a circle criterion type interpretation to Theorem 1 via the loop shifting theorem of Desoer and Vidyasagar[2.8]. Tet $M_{1}-I_{\text {be }}$ in the sector $(\alpha, \beta)$. Then for stabj]jty, we require that:

3. if $0<\alpha<\beta$, the Nyquist Jocus of $\frac{q^{-k} F B}{C(P R+Q A)}$ does not enclose or intersect the disc $\mathrm{D}\left[-\frac{1}{\alpha},-\frac{1}{\beta}\right]$

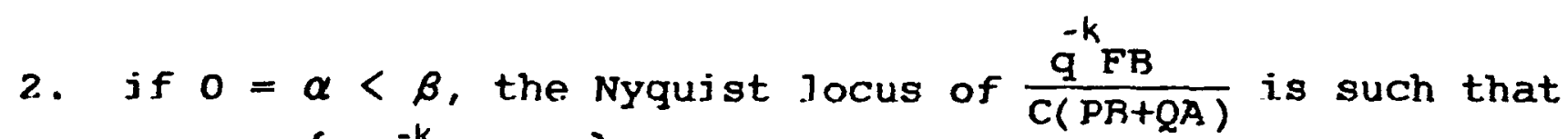
Rea] $\left\{\frac{q^{-k} F B}{C(P B+Q A)}\right\}>-\frac{2}{\beta} \forall|q|=1$

Note that we have assumed that $C(P B+Q A)$ has all roots inside the stable region.

An alternative formulation for the output error non-adaptive problem is to regard $e_{m}(t)$ as a function of $e_{1} \cdot+\hat{e}_{1}$. This will be useful later as a comparison with the adaptive case. 
ROBUSTNF.SS OF THF, NONADAPTIVF, CONTROTISFR

From F.gng. 32, 40 and 42. we get the error feedback system of Fig. 2-7 below.

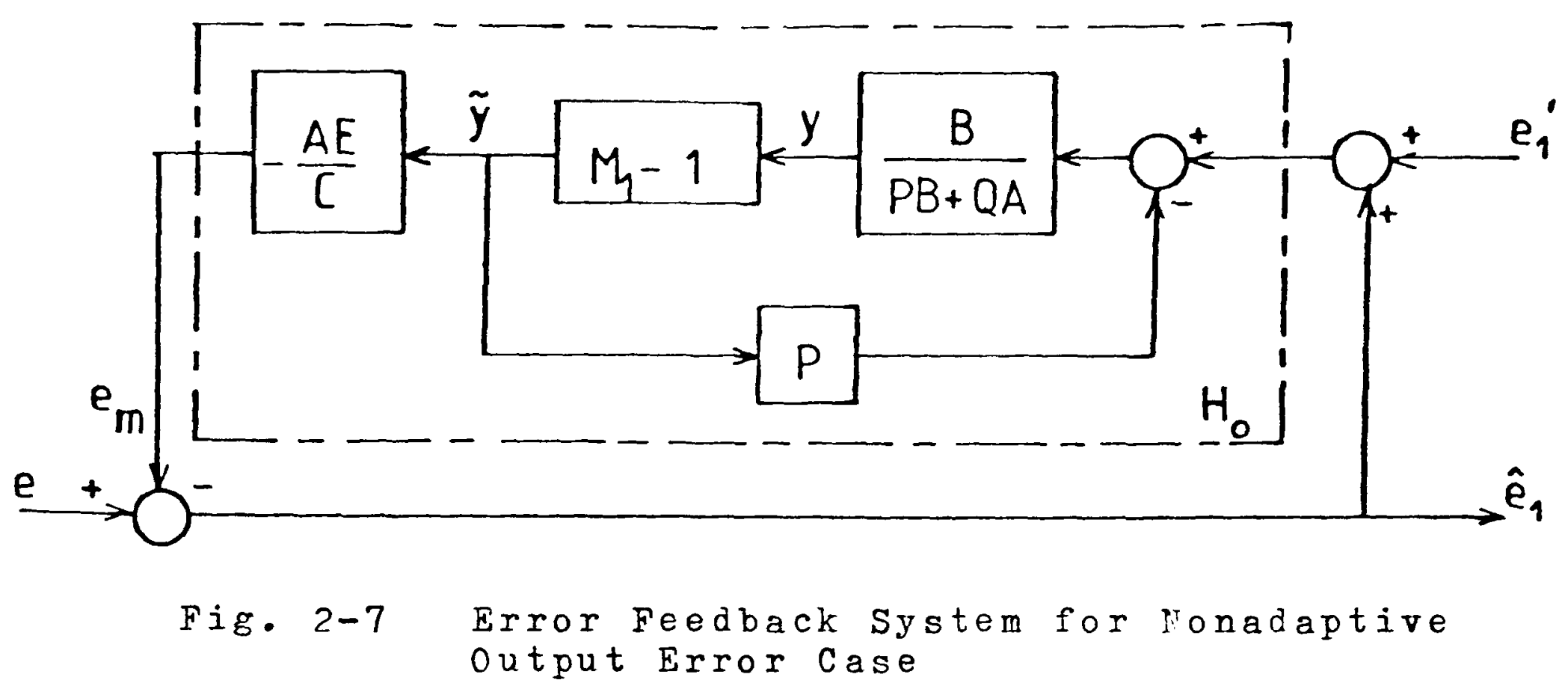

Define the blocks within the dotted line as the operator ${ }_{0}$

$$
\begin{aligned}
& \text { I.et } M_{1}-1=\bar{M}_{1} \\
& \text { and } P_{1}=\frac{P B}{P R+Q A}
\end{aligned}
$$

Then $H_{0}=-\frac{F A}{C} \ddot{M}_{1}\left[1+\dot{M}_{1} P_{1}\right]^{-1} P^{-1} P_{1}$

\section{Theorem 2}

The closed loop system of Fig. 2-7 is stable if

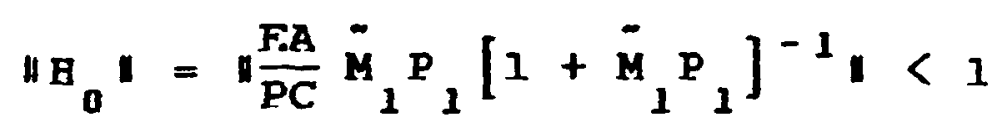

\section{Proof}

If operator $P$ is moved out of the inner loop to form $P$ in the feedforward path and $\mathbf{P}^{-1}$ is moved such that the inputs are now $\mathrm{P}^{-1} \mathrm{e}^{-1}$ and $\mathrm{P}^{-1} \mathbf{e}_{1} \cdot$, the result follows from the small gain theorem.

\section{Corollary 2.1}


ROBUSTNF.SS OF THF, NONADAPTIVF, CONTROT.T.F.R

If the model. reference design is used $(Q=0)$, and if $M_{1}^{-1}$ exists then for stabijity, we require that,

$$
\sup _{|q|=1}\left|\frac{E A}{P C}\right|<\frac{1}{\delta} \quad \text { where } \delta=\operatorname{gain}\left\{M_{1}^{-1}-1\right\}
$$

Proof.

Follows from F.qn. 49

$Q=0$ implies $P_{1}=1$

1. $+\ddot{M}_{1}=M_{1}$

then $\dot{M}_{1}\left[1+\ddot{M}_{1}\right]^{-1}=\left[M_{1}-1\right] M_{1}^{-1}=1-M_{1}^{-1}$

D

\section{Corollary 2.2}

If the model reference method is used $(Q=0)$, and if $M$, is linear then for stability we require $\frac{F \cdot A}{P C}\left[\frac{B A}{A B} 0-1\right]$ obeys the usual Nyquist criterion with respect to -1 .

\section{Proof}

Jet $M_{1}=\frac{A B}{B_{0}}$ in $B_{0}$ and apply Nyquist Theorem.

$\square$

\section{Corollary 2.3}

If $M_{1}$ is linear and $Q \neq 0$, then for stability we require that $\frac{E}{C}\left[\frac{A B_{0}-B A_{0}}{R B_{0}+Q A_{0}}\right]$ obeys the usual Nyquist criterion with respect to -1 .

Proof 
ROBUSTNF.SS OF THF. NONADAPTTVF. CONTROT.T.F.R

Substitute $M_{1}=\frac{A B}{R A}{ }_{0}$ in F.qn. 49

$\square$

Remark 1

The case of $M_{1}$ being nonlinear and non-invertible wi.ll be discussec in section 4.2

\section{Remark 2}

Corollary 2.3 shows that as in the linear full order case, $Q$ can serve to ensure stability if the true plant is non-minimum phase i.e. $B_{0}$ has unstable roots. Lassuming of course that $A_{0}$ and $Q$ are such that $P B_{0}+Q A$ is stable.)

\subsubsection{Input Error}

In this section, we assume that

$$
Y=0 \quad:\left(M_{1}=1, M_{2} \neq 1\right)
$$

Theorems 1-2 below offer a selection of conditions for stability in this case.

\section{Theorem I}

If $M_{2}$ is not necessarily invertible, then Treorem 2 of section 2.4.1 is applicable for $\varphi=0, \tilde{u} \neq 0$ with $M_{1}$ replaced by $M_{2}$. 
ROBUSTNF.SS OF THF, NONADAPTIVF, CONTROITIFR

\section{Remark 1}

This theorem implies that for robust stability, there is no difference between a nonlinearity at the output and a nonlinearity at the input.

\section{Remaxk ?.}

In the linear higher order case, there is of course no difference because operators in a linear time-invariant siso system commute. Hence remarks $1,2,4,5$ of Theorem 1 in section 2.4 .1 are valid here.

If $M_{2}^{-1}$ exists, then sjmilar manipulations to the output error case gives

$$
a(t)=\left(M_{2}^{-1}-I\right) \frac{A}{P B+Q A}\left[e_{2} \cdot(t+k)+e(t+k)-\left(Q+\frac{B F}{C}\right) \tilde{u}(t)\right]
$$

Define:

$$
G^{\prime} \cong \mathbf{E B}+Q C
$$

Eqns. 51 and 52. may be written as the feedback system of Fig. 2-8

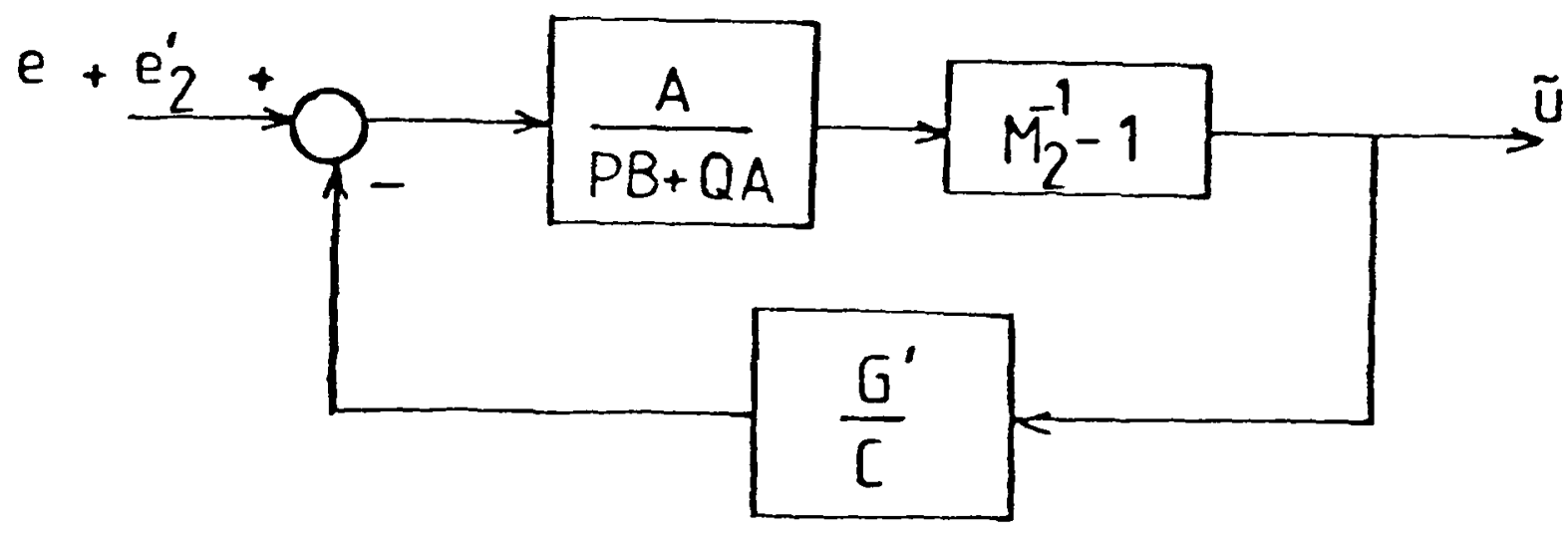

Fig. 2-8 Feedback System for Input Error 
ROBUSTNF.SS OF THF. NONADAPTIVE, CONTROI,T.F.R

Any of the following sets of conditions imply input-output stability of the system of Fig. $2-8$ if $M_{2}^{-1}$ exists.

a) $M_{2}$ is nonlinear and

$$
\begin{aligned}
& \sup _{|q|=1}\left|\frac{G \cdot A}{C(P B+Q A)}\right|<\frac{1}{6} \\
& \text { where } \delta=\operatorname{gain}\left(M_{2}^{-1}-1\right\}
\end{aligned}
$$

b) $M_{2}$ is linear and

$$
\left|\frac{A}{B_{0}}-\frac{A}{B}\right|<\left|\frac{C(P B+Q A)}{B^{\prime}}\right| \vee|q|=1
$$

c)

$\left[\frac{A}{B_{0}^{0}}-\frac{A}{B}\right] \frac{G^{\prime} B}{C(P B+Q A)}$ obeys the usual.

Nyquist encirclement condition.

Proof

As in proof of Theorem 1, section 2.4.1

口

The input error feedback system corresponding to Fig. 2-7 (for the output exrox case) is in Fig. 2-9 below.

Obviously Theorem 2 of Section 2.4 .1 is valid with $H_{0}$ replaced by ${ }_{i}$. Similarly, Corollaries 2.1-2.3 of Theorem 2, Section 2.4.1 are valid here with $M_{1}$ replaced by $M_{2}$. 
ROBUSTNF.SS OF THF. NONADAPTIVF. CONTROI.TIFR

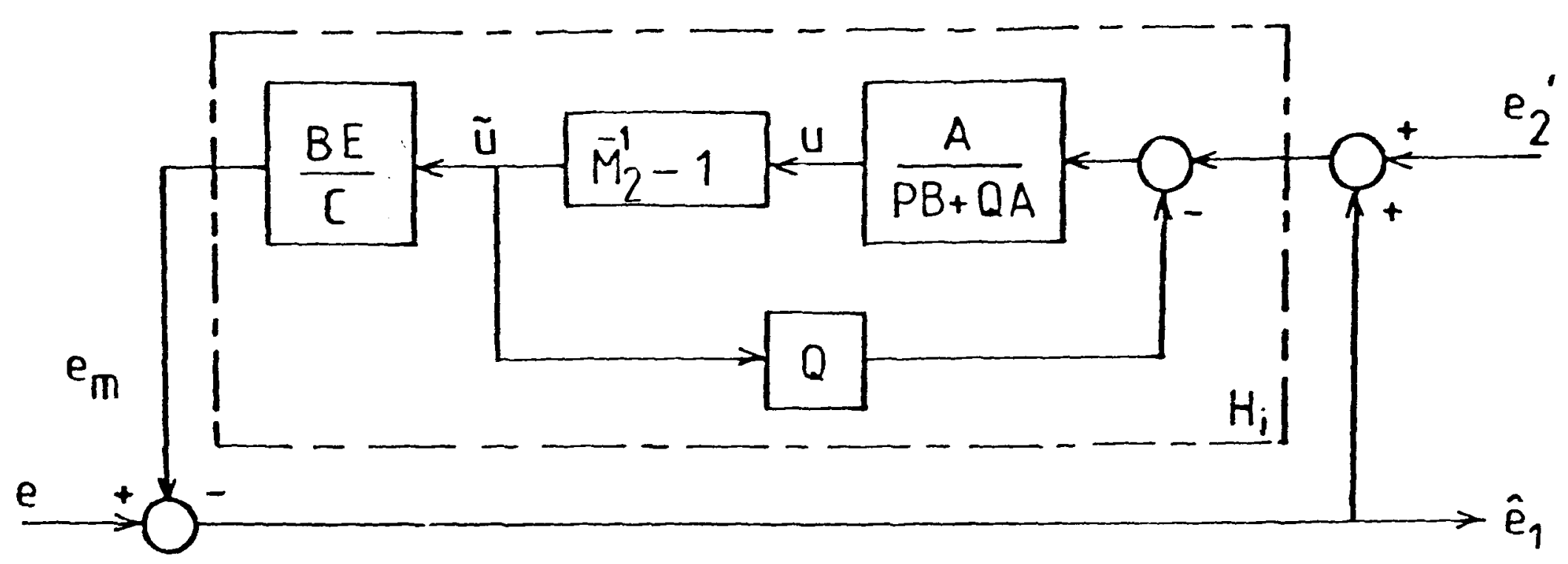

Fig. 2-9 Error Feedback System for Nonadaptive Input Error Case

\subsubsection{Discussion}

The results presented in sections 2.4 .1 and 2.4 .2 are for the robust stability of a specific design method. In essence, given a nominal plant $\frac{B}{A}$ and the maximum magnitude of plant perturbation, we can choose the design polynomials $P, Q, C$ to ensure stability. Next we examine an alternative interpretation for the design polynomial $c$ before looking at the robust stability properties of some other design methods.

\section{Observer-Controllex}

The control law for the non-adaptive case is given by

$$
(Q C+B E) u_{0}(t-k)=C R w(t-k)-q^{-k} F_{0}(t)
$$

If a state feedback controller using observed states was being used, it follows by a direct comparison of the control laws that the common factors of $C R$ and the closed loop characteristic polynomial correspond to the observer poles, Astrom[10], for the nominal plant. The closed loop characteristic polynomial of the nominal plant, $\frac{B}{A}$ with the control law of 
ROBUSTNF.SS OF THF, NONADAPTIVF, CONTROTI.F.R

F.gn. 53 above is

$$
\begin{aligned}
(Q C+B F) A+q^{-k} B F & =A Q C+B\left(F A+q^{-k} F\right) \\
& =C(P B+Q A)
\end{aligned}
$$

The common factors are the roots of $c$. Hence the roots of the $c$ polynomial i.e. the poles of the data vector filter may be regarded as the observer poles. The roots of $P B+Q A$ may then be regarded as the controller poles of the nominal closed loop system.

\section{IQQG design}

The robust stability design so far lacks generality in the sense that the resulting controllers are a function of nominal plant parameters. It is well known that for a continuous time linear time-invariant plant, an optimal controller designed to minimise a quadratic cost function and utilising full state feedback will have $\infty$ gain margin and at least $60^{\circ}$ phase margin. Anderson and Moore[5]. This can also be interpreted as allowing a nonlinear perturbation on the nominal input such that the nonlinearity $M_{i}$ is in the sector $\left(\frac{1}{2}, \infty\right)$. This result is independent of the nominal plant parameters and follows from the discovery by Kalman[52] of a frequency domain inequality for optimal controllers

$$
\left|1-K\left(s I-A_{s}\right)^{-1} B_{s}\right| \geqslant 1 \quad s=j \omega
$$

where $K$ is the controller gain and

$$
\begin{aligned}
& x(t+1)=A_{s} x(t)+B_{s} u(t) \\
& y=C_{s} x
\end{aligned}
$$

is a minimal state-space description of the system. 
ROBUSTNF.SS OF THF. NONADAPTIVF, CONTROI,T,F.R

However for discrete-time systems, it has been shown by Molander[68] that the discrete time equivalent of f.gn. 55 is not generally true. Instead we get an inequality that is a function of nominal plant parameters. Hence in discrete time, even the full state feedback optimal controller does not have the neat robustness properties of its continuous time counterpart.

In Kuo[55], Molander[68] it is shown that if the sampling period of a sampled data system tends to zero and if the sampled system is still minimum phase (eg. when $\operatorname{deg}(A)-\operatorname{deg}(B) \leqslant 1$ ) then the discrete time optimal controller tends in the limit to the continuous time optimal. controller. In such a case, the robustness properties of the full state IQG controller are recovered asymptotically as sampling period decreases.

However a further snag is that such robustness properties are only obtained when all the states of the system are accessib]e. Anderson[6], Molander[68], Kwakernaak[56], Doyle and stein[32]. A procedure for robustness recovery in minimum phase systems, when observers are used, is presented in Doyle and stein[32], which ensures that the poles of the observer tend asymptotjcally to the roots of

$$
\operatorname{det}\left[z I-A_{s}\right] \operatorname{det}\left[C_{s}\left(z I-A_{s}\right)^{-1} B_{s}\right]=B\left(q^{-1}\right)
$$

Bence the Doyle and stein observer which recovers full state robustness in this case has for poles the zeros of the open loop nominal system (assuming that these are stable and that $A_{\mathbf{s}}, \mathrm{B}_{\mathbf{s}}, \mathrm{C}_{\mathbf{s}}$ constitute a minimal realisation.) 
ROBUSTNF.SS OF THF. NONADAPTIVF. CONTROT.T.F.R

In the next two subsections we discuss the robust stabilities of the pole placement design methods which are the basis of the pole placement self-tuners of Wel]stead[85] and Astrom[11]. It is shown that the NA C-G STC can be designed to be as robust as the pole placement designs.

\subsubsection{Pole-Zero Placement (Astrom And Wittenmark[10],[11])}

In this and the next subsection, A, B, E, F, C, R are exactly those for the NA C-G STC. For this non-adaptive pole placement algorithm, the control law is

$$
R_{A}\left(q^{-1}\right) u_{0}(t)=T_{A}\left(q^{-1}\right) w(t)-s_{A}\left(q^{-1}\right) Y_{0}(t)
$$

The reros of the nominal plant are factorised as

$$
B=B^{+} B^{-}
$$

where $\mathrm{B}^{+}$are the open loop zeros not wanted in the closed loop and hence to be cancelled, and

$B^{-}$are the open loop zeros that cannot be cancelled or are desired in the closed loop.

The control law polynomials are selected as

$$
\begin{aligned}
& R_{A}=R_{1} B^{+} \\
& T_{A}=C R
\end{aligned}
$$

where $R$ contains the desired closed loop zeros not available in the open loop plant

$C$ contains the observer poles as in the NA C-G STC and $R_{1}$ and $S_{A}$ are obtained from the solution of

$$
C A_{m}=A R_{1}+q^{-k_{B}-S_{A}}
$$

$A_{m}$ being the desired closed loop pole polyuomial 
ROBUSTNF.SS OF THF, NONADAPTIVF, CONTROIIT.F.R

In Appendix A.5 it will be shown that in the output error case F.gns. 58 to 62 result in the error feedback system of Fig. 2-10 below.

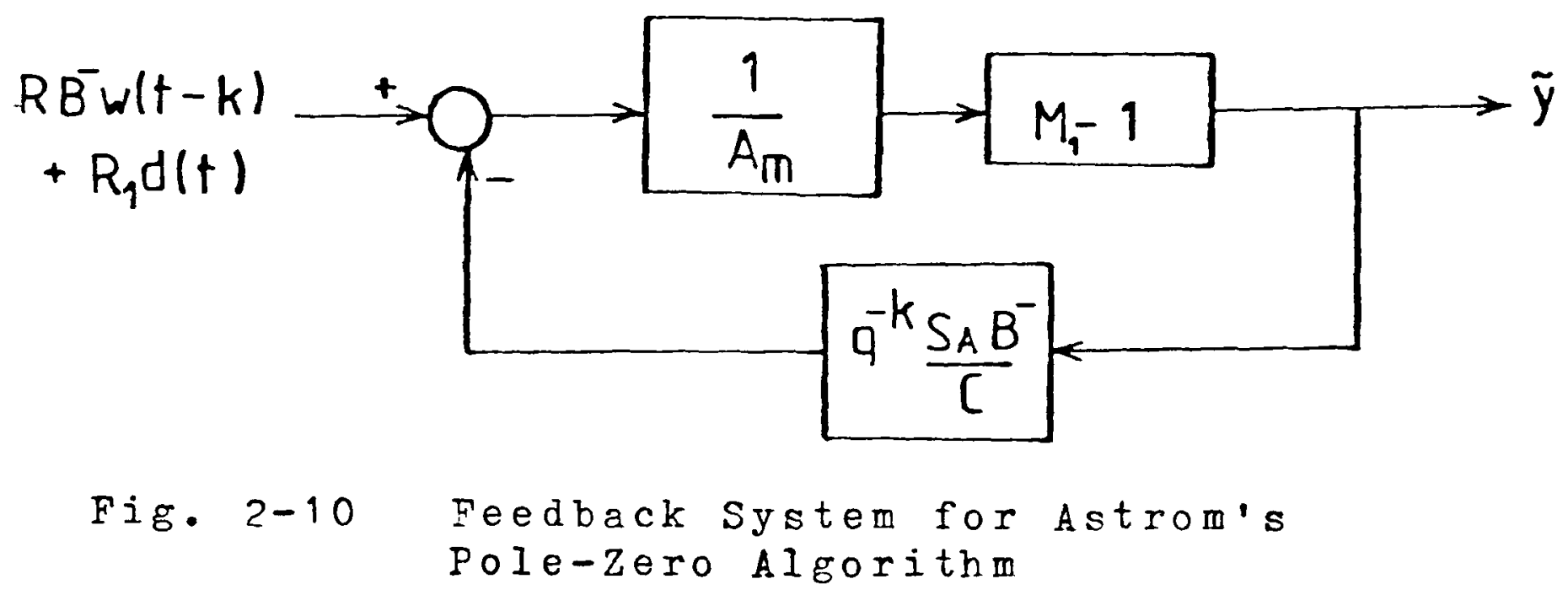

If all the process zeros can be cancelled i.e. $B^{-}=1$ and if $\operatorname{deg}\left\{R_{1}\right\}$ in the solution of Fqn. 62 is chosen to be $k-1$, then the criteria for stability of the feedback system of Fig. 2-10 is exactly that for the NA C-G STC controller considered in section 2.4.1 with $Q=0$ (model reference design method). This is easily seen by replacing $R_{1}$ by $E$ and $S_{A}$ by $F$ in Fqn. 62 and comparing with F.qn. 14 (the identity for the NA C-G STC).

However there are many other possible solutions for $\mathbf{R}_{\mathbf{1}}$ and $\mathbf{S}_{\mathbf{A}}$ in Eqn. 62. and hence many possibilities for controller design to ensure robustness. BY comparison the NA C-G STC allows only a unique solution to F.qn. 14. On the other hand, the NA C-G STC has an extra design variable in $Q$, which can be used as in Theorem 1 . 


\section{Theorem 1}

To every pole-zero placement controller resulting from the solution of F.qn. 67., if $P=A_{m}=$ desired closed loop poles, there exists a $Q$ such that the corresponding NA C-G STC has exactly the same robustness properties.

Proof

In Appendix A.5

Remarks

Theorem I does not necessarily imply that the resulting control laws are identical. The control law for the non-adaptive pole-zero placement algorithm is, with the $Q$ selected as in Theorem 1

$u_{0}(t)=\frac{R C}{B \cdot R_{1}} w(t)-\frac{F}{B F_{1}+Q C} y_{0}(t)$

Compare with the control Jaw for the NA C-G STC:

$$
u_{0}(t)=\frac{R C}{B F_{2}+Q C} w(t)-\frac{F}{B F_{i}+Q C} y_{0}(t)
$$

In F.qn. 63, the poles of the setpoint filter contain only the stable zeros of B, while in Eqn. 64 the unstable zeros of B are included but 'neutralised' by QC.

The theorem is of no practical importance in the adaptive case. Here it serves to demonstrate that the design methods that are the basis for Astrom's pole-zero self-tuner and the clarke-Gawthrop self-tunex are equivalent as far as robust stability is concerned. 
ROBUSTNF.SS OF THF, NONADAPTIVF, CONTROT,T.F.R

2.4.5 Pole Assignment (Wellstead Ft Al[B5],[86])

The main-feature that distinguishes this algorithm from the preceding one is that all open loop zeros are allowed to remain in the closed loop. In consequence, this method is supposed to be more robust to non-minimum phase zeros than the NA C-G STC. This algorithm is also desjgned for regulator as well as for the servo performance of the previous section. Desired closed loop zeros which are not open loop zeros are included via a prefilter on the setpoint and are hence not available in the regulator. An advantage of this method is that there is no need to factorise polynomials.

The control law is obtained from

$$
G_{s} u(t)=H_{s} w(t)-P_{s} y(t)
$$

where

$G_{s}$ and $F_{s}$ are obtained from the solution of

$A G_{s}+F_{s} \bar{B}=C A_{m}$

and $\bar{A}=q^{-k} B$

B may contain leading zero coefficients to account for unknown delays.

A contains the desired closed loop poles.

$C$ may be interpreted as the data vector filter or as the estimated noise polynomial.

Appendix A.6 shows that the corresponding error feedback system is Fig. 2-11 below (output error case: $0=0$ )

Comparing with Fig. 2-5, it is obvious that stability of this feedback system is assured if

$$
\sup _{|q|=1}\left|\frac{F}{\operatorname{C}} q_{m}^{\tilde{B}}\right| \operatorname{gain}\left\{M_{1}-1\right\}<1
$$




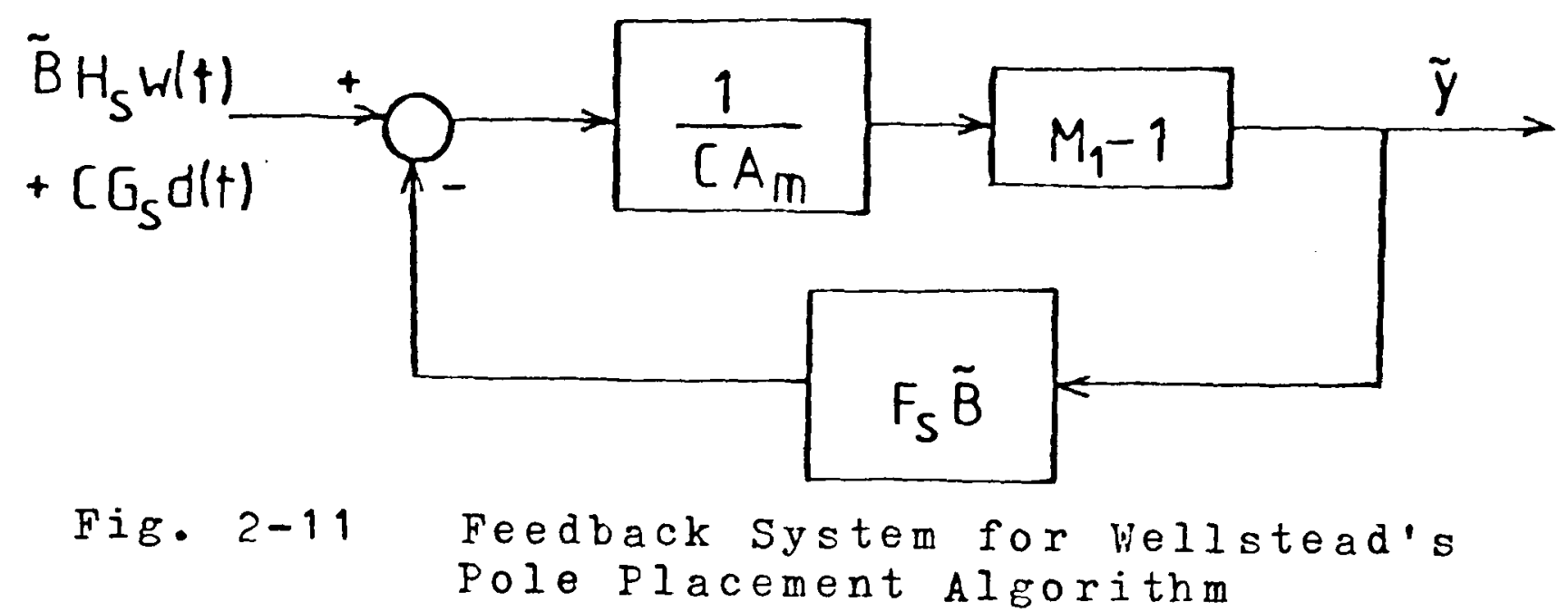

Theorem I

For every pole assignment controller designed from Eqns. 65 to 68 , there exists a NA C-G STC with $P=A_{m}$ and $Q=\frac{P}{A}\left(\frac{F}{F_{g}}-B\right)$ which has identical robustness properties.

\section{Proof}

By direct comparison of Egn. 69 and section 2.4.1

口

\section{Remarks}

As in the previous section, Theorem 1 does not force the control laws to be identical. It demonstrates that in the non-adaptive case, the choice of the $P$ and $Q$ specified ensures that the roots of. $P B+Q A$ are the roots of PF which does not include the non-minimum phase roots of $B$.

Unlike the pole-zero placement controller of the preceding section, the controllex polynomials here are forced to be unique by fixing the degree of $F_{\mathbf{s}^{\prime}} \mathbf{G}_{\mathbf{s}}$ in F.qn. 66 . Hence to each $F_{\mathbf{s}^{\prime}} G_{\mathbf{s}}$ and $P=A_{m}$, there exists a unique $Q$ to ensure ${ }_{n}$ robust stability. 
ROBUSTNF.SS OF THF, NONADAPTIVT, CONTROTIT.F.R

\subsubsection{Instability of The Non-adaptive Controller}

In the preceding sections, where the Nyquist theorem has been used to show stability of error feedback systems, it has been possible to show instability at the same time, because the Nyquist theorem provides necessary and sufficient conditions.

Where plant perturbations are nonlinear, the stability theorems are only sufficient. It j.s well known that these theorems are quite conservative. It is thus desirable to discover sufficient conditions for instability, so that where possible, they serve as a complement and a guide to the conservativeness of the stability theorems.

The error feedback system is said to be $1_{2}$ unstable if there exists any input in $]_{2}$ such that the output is not in $1_{2}$.

Consider the output error case. Fig. 2-5 may be redrawn as Fig. 2-12 below.

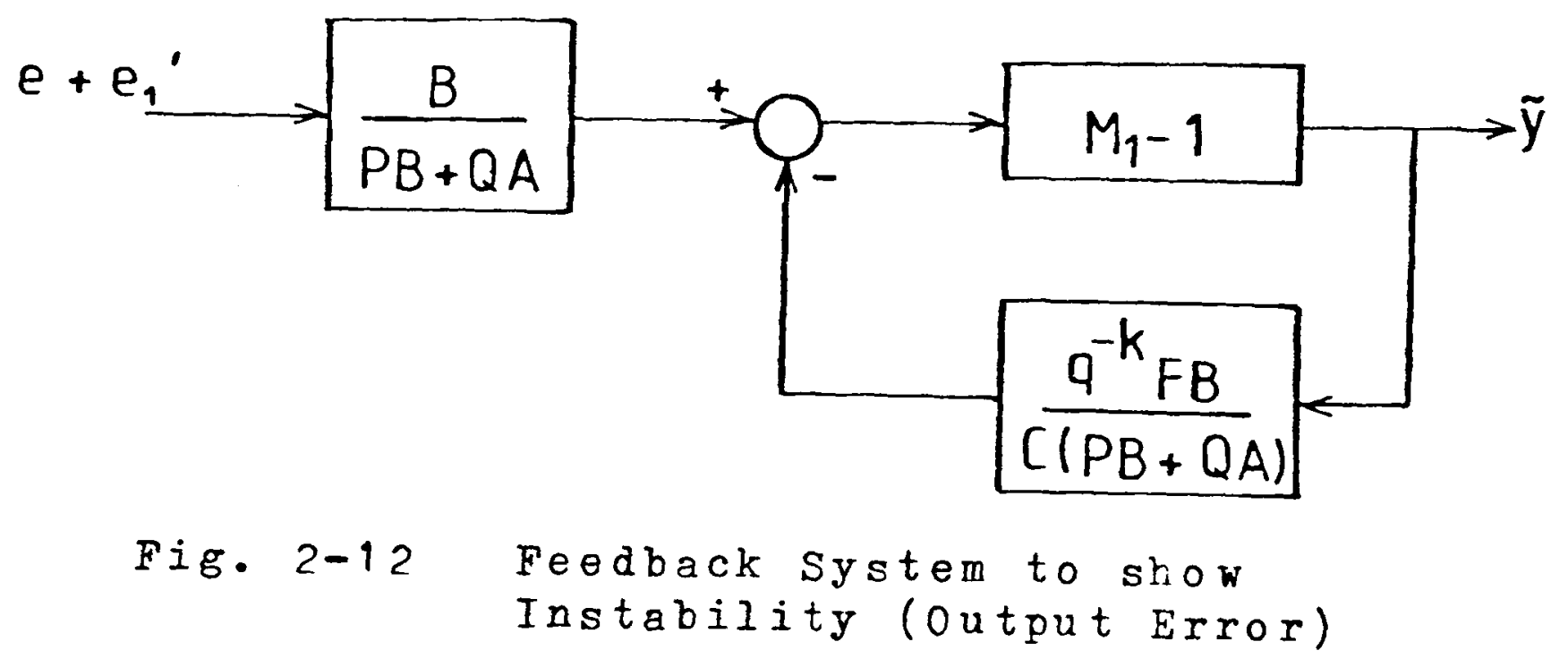


ROBUSTNF.SS OF THF. NONADAPTIVF, CONTROI,T.F.R

A standard result for $1_{2}$ instability of this feedback system is stated bejow.

\section{Theorem I}

In the feedback sygtem of Fig. 2-12. above, if [M -1$]$ is a time-invarjant, memoryless operator in the sector $(\alpha, \beta): 0<\alpha<\beta$, and

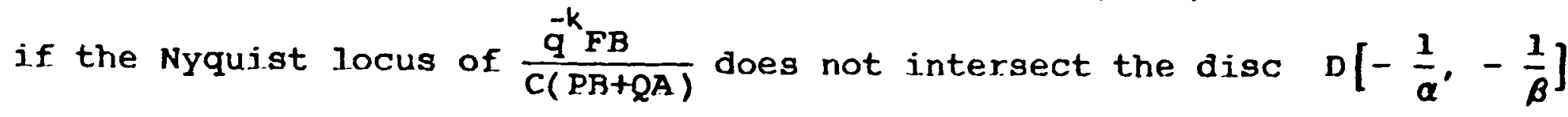
and encircles it at least once in the clockwise direction, then there exists some input $\left[e(t)+e_{1} \cdot(t)\right]$ in $I_{2}$ such that $y_{1} y I_{2}$

\section{Proof:}

Desoer and Vidyasagar[28] for input-output approach. Brockett and Inee[17] have a version derived using the methods of Jyapunov stability.

\section{Remark 1}

This theorem is complementary to the circle criterion of remark 5 , Theorem 1, section 2.4.1. Note that $C(P B+Q A)$ is assumed to have all roots stable - hence Theorem 1 is a more restricted version of the general theorem.

In the input error case, we have (from Appendix A.4) the feedback error system of Fig. 2-13 below.

\section{Corollary 1}




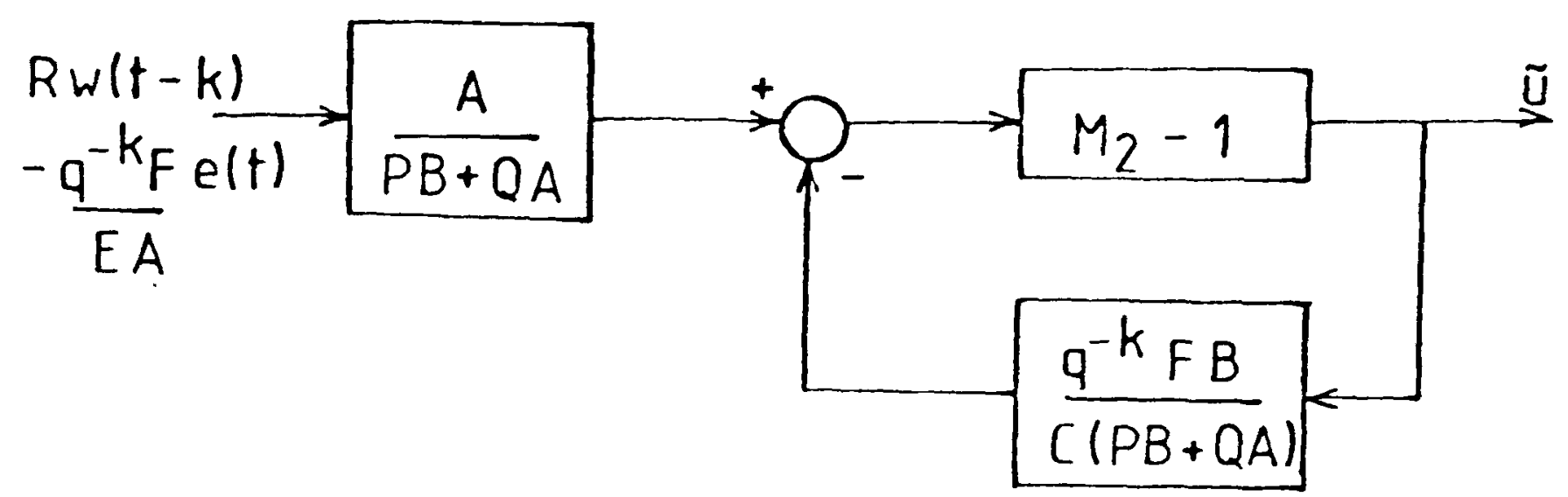

$\begin{aligned} \text { Fig. 2-13 } & \text { Feedback System to show Instability } \\ & (\text { Input Error) }\end{aligned}$

Theorem I is equally applicable to the error feedback system of Fig. 2-13 except that the input is now $R w(t-k)-q^{-k F} \frac{k A}{F A}(t)$

Both Theorem $I$ and Coroliary $I$ are restrictive in that a sector bound is required for $M_{1}-1$. The stability results of the previous sections only required an upper bound on the possible plant perturbations. In addition, $1_{2}$ instability is a rather weak form of instability in this context because it includes eg. the cases when $\$$ or a (in Figs.12 and 13) have a non-zero mean. This would be likely especially if the setpoint, $w(t)$ has a non-zero mean.

A stronger form of instability is $1_{\infty}$ instability. In Chapter 3 , the technique of exponential weighting will be used to develop $1_{\infty}$ instability results from $1_{2}$ instability results. [see sections 3.4 and 3.5 ]. Here the result will only be stated for Theorem 1. above. 
RORUSTNF.SS OF THF. NONADAPTTVF. CONTROIJT.R.R

For a linear ARMA model, $I,\left(q^{-1}\right)$, define its exponentially weighted associate as

$$
\begin{aligned}
J_{1}^{*}\left(q^{-1}\right) & \doteq I_{1}\left([\exp (\gamma) q]^{-1}\right) \\
& =\exp (-\gamma t) I_{1}\left(q^{-1}\right) \exp (\gamma t)
\end{aligned}
$$

Corollary 2 (1 counterpart of Theorem 1)

In the feedback system of Fig. 2-12 above, if $M_{1}-1$ is a time-invariant, memoryless operator in the sector $(\alpha, \beta): 0<\alpha<\beta$ and if there exists an $\gamma>0$ such that the Nyquist locus of the exponentially weighted associate, $\left[\frac{\mathrm{q}^{-k} \mathrm{FB}}{\mathrm{C}(\mathrm{PB}+Q \mathrm{QA})}\right]^{\text {* }}$ does not intersect the disc $\mathrm{D}\left[-\frac{1}{\alpha},-\frac{1}{\beta}\right]$ and encircles it at least once in the clockwise direction, then there exists some input $\left[e+e_{1}\right] \in I_{\infty}$ such that $y, 9 \notin I_{\infty}$.

Proof

Similar to Section 3.4 and 3.5 .

Remarks

Corollary 2 may be viewed as the strong instability counterpart of Theorem 1. Note that for the corollary to be true, the exponentially weighted associate of $[P B+Q A]$ must still be stable. From the definition of an associate operator given above, if $[P B+Q A]$ has a root at $q=\lambda$ then the associate has a root at $q=\exp (\gamma) \lambda$. Not surprisingly therefore, the conditions of Theorem 1 are 'easiex' to meet than those of Corollary 2 
ROBUSTNF.SS OF THE NONADAPTIVF, CONTROTIIFR

\subsection{ROBUST DISTURBANCE, ATTF.NUATION: NON-ADAPTIVF, CONTROTI.F.R}

In the linear full order NA C-G STC, it is easy to show that $\hat{e}_{1}(t)=F d(t)=e(t)$, where $F\left(q^{-1}\right)$ is a polynomial in $q^{-1}$ of degree $k-1$, and $e(t)$ represents the disturbances which cannot be rejected by a causa] control law. If $d(t)$ were a white noise process, then $e(t)$ is a moving average process of order $k-1$. In this section, the $c$ polynomial will be assumed to be designed first for maximum disturbance rejection in the nominal plant. $P$ and $Q$ are thus designed to cope with the plant perturbation. This is logical because in the solution of the design identity, Eqn. I4, F will depend on the observer polynomial $C$. Hence $e(t)$, the maximum residual disturbance in the nominal plant depends on $c\left(q^{-1}\right)$ too.

In standard stochastic control theory, if the disturbance could really be modelled as $\frac{C}{A} d(t)$ with $d(t)$ a white noise process, we get optimum disturbance rejection. In the linear full order self-tuning controller[20], $C$ is estimated. Here $C$ is an arbitrary design polynomial which may be chosen for robust stability requirements rather than disturbance rejection. Note also that in the linear full order NA C-G STC this disturbance rejection property holds irrespective of whether the controller is also required to track a non-zero setpoint.

In this section, the disturbance rejection properties of the controller are examined in the presence of plant perturbations which are not necessarily linear. 
ROBUSTNF.SS OF THF, NONADAPTIVF, CONTROT,T.F.R

\subsubsection{Feedback system And Fquations}

The output and input error cases will be considered together.

Fig. 2-14 below is similar to Fig. 2-7 for the output error case and Fig. 2-9 for the input error case.

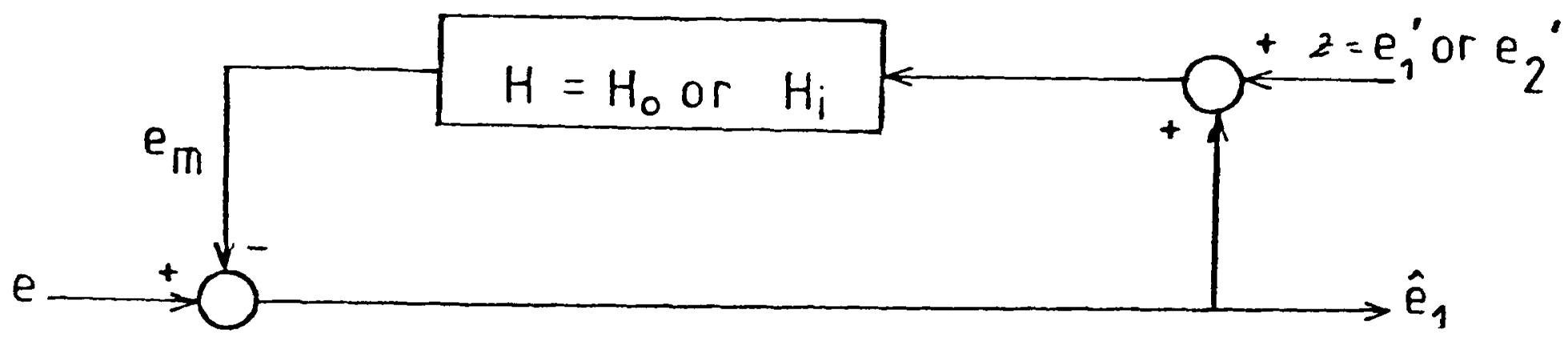

Fig. 2-14 General Error Feedback System

In the output exror case,

$$
H=H_{0} \quad \text { and } \quad z=R w(t-k)+\frac{C Q}{B} \mathrm{~d}(t)
$$

In the input error case,

$$
H=H_{i} \text { and } \quad z=R w(t-k)-\frac{C P}{A} \alpha(t)
$$

Define $\ddot{B} \cong \mathbf{H}(1+H)^{-1}$

Then $\hat{e}_{1}=-\dot{H}\{e+z\}+e$

Note that $\overline{\mathrm{H}}$ is not necessarily linear. If there is no plant perturbation,

$$
\dot{H}=0 \text { and } \hat{e}_{1}(t)=e(t) .
$$

\subsubsection{Theorems For Disturbance Attenuation}

Theorem 1 
If the plant perturbation is linear, then minimising the gain of the closed loop operator, $\tilde{\mathrm{B}}=\mathrm{H}(\mathrm{I}+\mathrm{H})^{-1}$, at each frequency maximises attenuation of $(e+z)$, regarded as a disturbance, at the frequency. Proof

Note that the controller seeks to minimise $\hat{e}_{1}-e$. Theorem 1 is obvious from Eqn. 73 above.

Remarks

First consider $z=0$. This excludes the input error case. In the output error case, $z=0$ if setpoint, $w=0$ and $Q=0$.

The result of Theorem 1 then differs slightly from standard sensitivity theory, where it is the inverse return difference function, $(1+\mathrm{H})^{-1}$, whose gain is usually minimised. Here we note that from Eqn. 73 and with $z=0$, we can get

$$
\begin{aligned}
\hat{e}_{1}(t) & =-\dot{B}\{e\}+e \\
& =(1+B)^{-1} e(t)
\end{aligned}
$$

Hence it is also possible to seek to minimise the gain of $(1+\mathrm{H})^{-1}$ at the frequency of the disturbances, but this is less logical in view of the fact that the nominal controller is designed to minimise $\hat{e}_{1}(t)-e(t)$. rather than $\hat{e}_{1}(t)$. Note however that in Corollary 2.2 of section 2.4.1, we required that for stability, the Nyquist plot of $\frac{E}{P C B}\left(A_{0} B-B_{0} A\right)$ does not encircle the $-1+j 0$ point in the complex plane. If in the same complex plane, a circle of radius 1 and centre $(-1,0)$ is drawn, then for all frequencies where the Nyquist plot is outside the circle,

$$
\left|\left(1+\theta_{0}\right)^{-1}\right|<1
$$


and hence disturbances at those frequencies are attenuated despite the plant perturbations.

When the perturbation is linear,

$$
\tilde{H}=\frac{E\left(A_{0} B-B_{0} A\right)}{P\left(B_{0}+E\left(A_{0} B-B_{0} A\right)\right.}
$$

Prom Theorem I, P should be designed so that the magnitude of $\ddot{H}$ is small over the frequency range of the disturbances. This can be done graphically with the aid of M circles and the Nyquist ]ocus of $B$. [The use of $M$ circles is a traditional method for obtaining closed loop frequency response, (i), from the open loop frequency response i.e. the Nyquist locus of $\mathrm{H}$. See eg. Ogata[71]. They represent the loci of constant magnitude]. An example will illustrate this in the next section.

If $z \neq 0$, then there is an extra disturbance $z$ to be filtered by $\dot{B}$ from the error, $\hat{e}_{1}-e$. As $z$ containg the setpoint, which will usually be uncorrelated with $e$, we can expect that the effect of plant perturbation will be worse with $z \neq 0$.

For linear plant perturbations, it is possible to consider the inputs $e, z$ separately. In the nonlinear case, $\vec{B}(e+z) \neq \dot{B}\{e\}+\dot{B}\{z\}$ and we have

\section{Theorem 2}


ROBUSTNESS OF THF, NONADAPTIVE, CONTROIIIFR

If the plant perturbation is (a) nonlinear and (b) continuously Frechet differentiable, then

$$
\begin{aligned}
& \hat{e}_{1}(t)-e(t)=\dot{B e}-\int_{0}^{1} \dot{D H}(e+\alpha z) z d \alpha \\
& \text { where } \overline{D H} \text { is the Frechet derivative of } \dot{\mathrm{B}} \text {. }
\end{aligned}
$$

Proof

From F.qn. 73

$$
\hat{e}_{1}(t)-e=-\tilde{B}\{e+z\}
$$

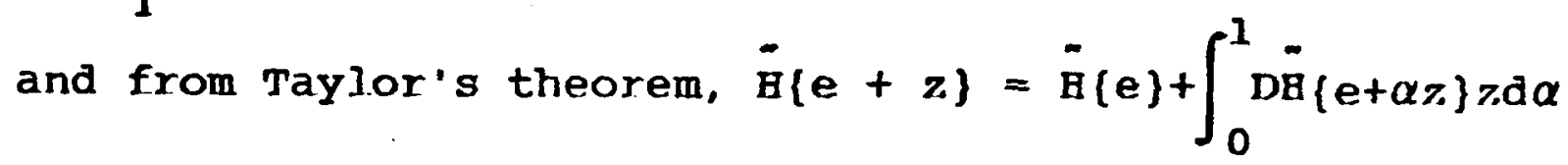

\section{Remark 1}

If $z=0$, then as in the linear case, $\bar{B}$ should be minimised for maximum disturbance attenuation. Note that

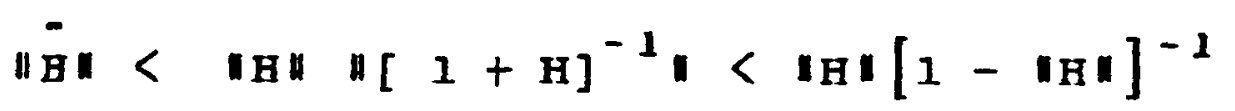

From Section 2.4, we have required that $\|$ B $<1$ for robust stability. Bence since $Q=0(z=0)$, we again design to ensure that $\sup _{|q|=1}\left|\frac{\text { F.A }}{C P}\right|$ is as small as possible, just as in the case of linear perturbations.

\section{Remark 2}

If $z \neq 0$, a reasonable design strategy would be to minimise le 1 , as well as $\ddot{H}(e)$, where

$$
\begin{gathered}
e_{z}=\int_{0}^{1} \operatorname{DB}\{e+\alpha z\} z d \alpha: 0 \leqslant \alpha \leqslant 1 \\
\operatorname{le}_{z} \leqslant \sup _{\alpha} \| \text { DA }\|z\|
\end{gathered}
$$

In the output error case, if $Q=0$,

$$
\begin{aligned}
& H=\frac{F A}{C}\left(H_{1}^{-1}-1\right) \frac{1}{P} \\
& D H=\frac{F A}{P C}\left[D\left(H_{1}^{-1}\right)-1\right]
\end{aligned}
$$


ROBUSTNF.SS OF THF. NONADAPTIVF, CONTTROI,I.F.R

$$
\begin{aligned}
\sup _{\alpha}\|D B\| & =\sup _{|q|=1}\left|\frac{F . A}{C P}\right| \sup _{\alpha}\left\|\left(M_{1}^{-1}\right)-1\right\| \\
& =\frac{F . A}{P C}\left\|M_{1}^{-1}-1\right\|_{\Delta}
\end{aligned}
$$

where ". $\Delta$ is the incremental norm.

$$
\begin{aligned}
\tilde{D H} & =\mathrm{D}\left[\mathrm{H}(1+\mathrm{H})^{-1}\right] \\
& =(\mathrm{DH})[x+\mathrm{DH}]^{-1}
\end{aligned}
$$

since DH is a linear operator, it is again possible to use $M$ circles to design for minimum in from the Nyquist locus for $D H$.

Bence minimising norm of $\frac{F A}{P C}$ minimises $\ddot{B}\{e\} "$ as well as $1 e_{z}$. Note that this does not necessarily mean that $\hat{e}_{1}$ is the minimum possible. Note also that it is impossible to ensure that the norm of $(1+B)^{-1} \leqslant 1$ if we are minimising $\|B\|$. Hence the upper bound on $\| \hat{e}_{1}$ is always greater than that for the unperturbed case. This problem has been addressed by 7ames[95] in his study of optimal sensitivity for linear systems.

If $Q \neq 0$, $\mathrm{NH}$ is more difficult to evaluate and the problem is deferred to Chapter 4.

Theorem 2 can be viewed as extending the standard sensitivity results for linear systems to the nonlinear case. (see [24],[25],[29] for recent general sensitivity results). Note that there is no requirement for the plant perturbation to be small. Theorem 2 specialises to Theorem 1 when the perturbation is linear. (The Frechet derivative of a linear operator is the linear operator itself).

\subsubsection{Setpoint Following}

self-tuners designed for tracking have been developed in Astrom[10],[11] and in Wellstead et al[87]. In the former, disturbanceg are assumed absent. The clarke-Gawthrop self-tuning controller by 
ROBUSTNESS OF THF. NONADAPTIVF, CONTROT.T.F.R

contrast is designed to give simultaneous tracking and regulation. In the previous section, it has been shown that disturbance attenuation will usually be worse with a non-zero setpoint than without. Here we have

\section{I.emna I}

If $Q=0$, the design criteria for robust disturbance attenuation i.s also that for minimising the variation of filtered output, $\Phi$, around the filtered setpoint, $R w(t-k)$.

\section{Proof}

From the control law in F.qn. 26 and the definition of prediction $\operatorname{error} \hat{e}_{1}$, we have

$$
\Phi(t)-R w(t-k)=\hat{e}_{1}(t)
$$

Note that this is true for both input and output error cases.

Robust tracking for continuous time systems has been studied in Desoer and wang[30] among others. However similax ana]ysis wi]l not be attempted in this section.

\subsection{EXAMPJ.ES}

In this section, two examples will be used to illustrate the results of the chapter. In the first, a problem of reduced order modelling is considered and in the second, design of a nominal plant to cope with possible nonlinearities is examined. 
RORUSTNF.SS OF THF, NONADAPTIVF, CONTROTIT.R

\subsubsection{Reduced Order Jinear Dynamics}

Consider the continuous time multiplicatively perturbed plant,

$G_{0}(s)=G_{1}(s)\left[1-G_{2}(s)\right]$, where

$G_{1}(s)=\frac{2}{s-1}:$ nominal plant

$G_{2}(s)=\frac{\mu s}{\mu s+1}:$ multiplicative perturbation

Note that the plant is open loop unstable. If $\mu$ is small, $G_{1}(\mathbf{s})$ represents a nominal plant which neglects a fast pole. This example is a.lso used in Sandell[77] to demonstrate the robust stability of a unity feedback system under singular perturbation. Assume that a discrete time NA C-G STC is used and control is applied via a zero order hold at a sampling perjod of 0.1 secs. The discrete nominal plant is

$$
G_{1}\left(q^{-1}\right)=\frac{0.2103 q^{-1}}{1-1.105 q^{-1}}
$$

If minimum variance control is used, i.e. $P=1, Q=0$ and if $c=1$,

Fig. 2-15 shows the Nyquist plots of $A=\frac{F\left(A_{O} B-B_{0} A\right)}{P C B_{0}}$.

By Corollary 2.2 of section 2.4.1, Fig. 2-1.5 shows that if $\mu<0.5$, the closed loop system is stable under minimum variance control.

From section 2.5.2, we get

$$
\hat{e}_{1}-e=\tilde{H}\{e(t)+w(t-k)\} \text {. }
$$

where

$$
\dot{H}=\mathrm{H}(1+\mathrm{H})^{-1}
$$

If setpojnt $w=0, \hat{e}_{1}=(1+H)^{-1} e(t)$.

Not surprisingly, $\ddot{B}$ increases as $\mu$ increases from 0 to 0.5 . Fig. 2-15 also shows the M circles from 0.2 to 1.0 . The loci for $|\ddot{B}|=1$ is a vertical line through $\frac{1}{2}$ i.e. an $M$ circle of infinite radius. obviously, to get the largest frequency range over which $|\ddot{A}|$ is less than 
ROBUSTNF.SS OP THF. NONADAPTIVF. CONTROT,T.F.R

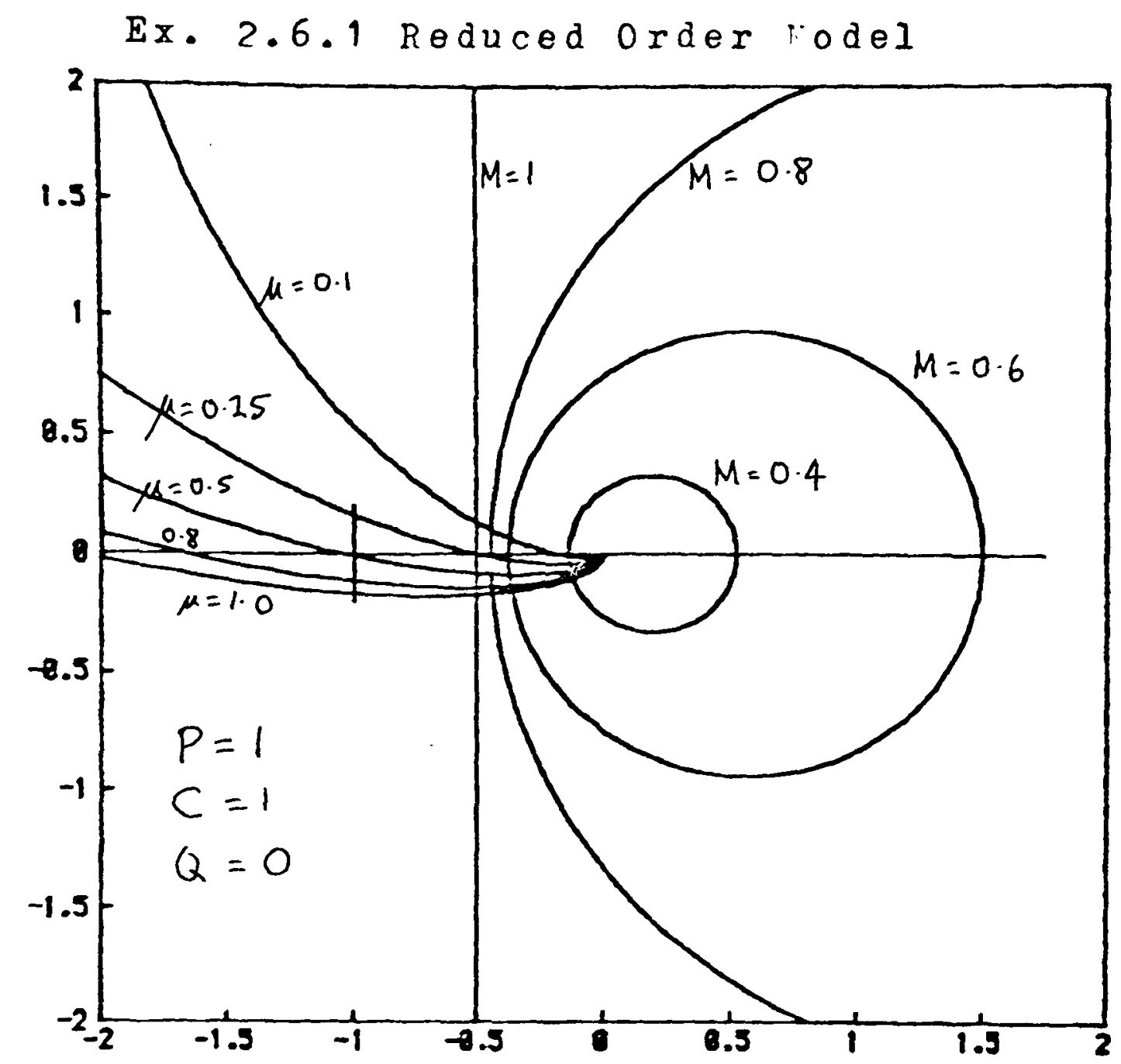

Fig. 2-15 Nyquist Loci for Varying perturbation

some arbitrary value, as much as possible of the Nyquist locus of $B$ should be to the right of $\frac{1}{2}+j o$. For convenience, let that arbitrary value be 1 . When $\mu=0.1$, the frequency ranges for which $|\overline{\mathrm{H}}|>1$ is

$$
0.108 \omega_{\mathrm{g}}<\omega<0.379 \omega_{\mathrm{s}} \text { and } 0.621 \omega_{5}<\omega<0.892 \omega_{\mathrm{s}} \text {. }
$$

When $\mu=0.5$, this has expanded to

$$
0.046 \omega_{\mathrm{s}}<\omega<0.461 \omega_{\mathrm{s}} \text { and } 0.539 \omega_{\mathrm{s}}<\omega<0.954 \omega_{\mathrm{s}}
$$

Bence the larger the perturbation, the more high frequency disturbances are amplified. It is also interesting in this case to look at the magnitude, $\mid(I+H)^{-1}$ e $\mid$ for $e$ of varying frequency.

If $\mu=0.1,\left|(1+\mathrm{H})^{-1}\right|<1$ for frequency $\omega$ such that 
RORUSTNF.SS OF THF NONADAPTIVF, CONTROT,I.F,R

$0.19 \omega_{8}<\omega<0.81 \omega_{8}$ where $\omega_{8}$ is the sampling frequency. This implies that the perturbed system attenuates disturbances in that frequency range more than the unperturbed system would. As $\mu$ increases, this range actually increases and at $\mu=0.5$, we get

$0.095 \omega_{s}<\omega<0.905 \omega_{s}$. Note however that being a sampled system, this is a recurring range i.e. it is true for

$0.095 n \omega_{s}<n \omega<0.905 n \omega_{s}$ (n an integer).

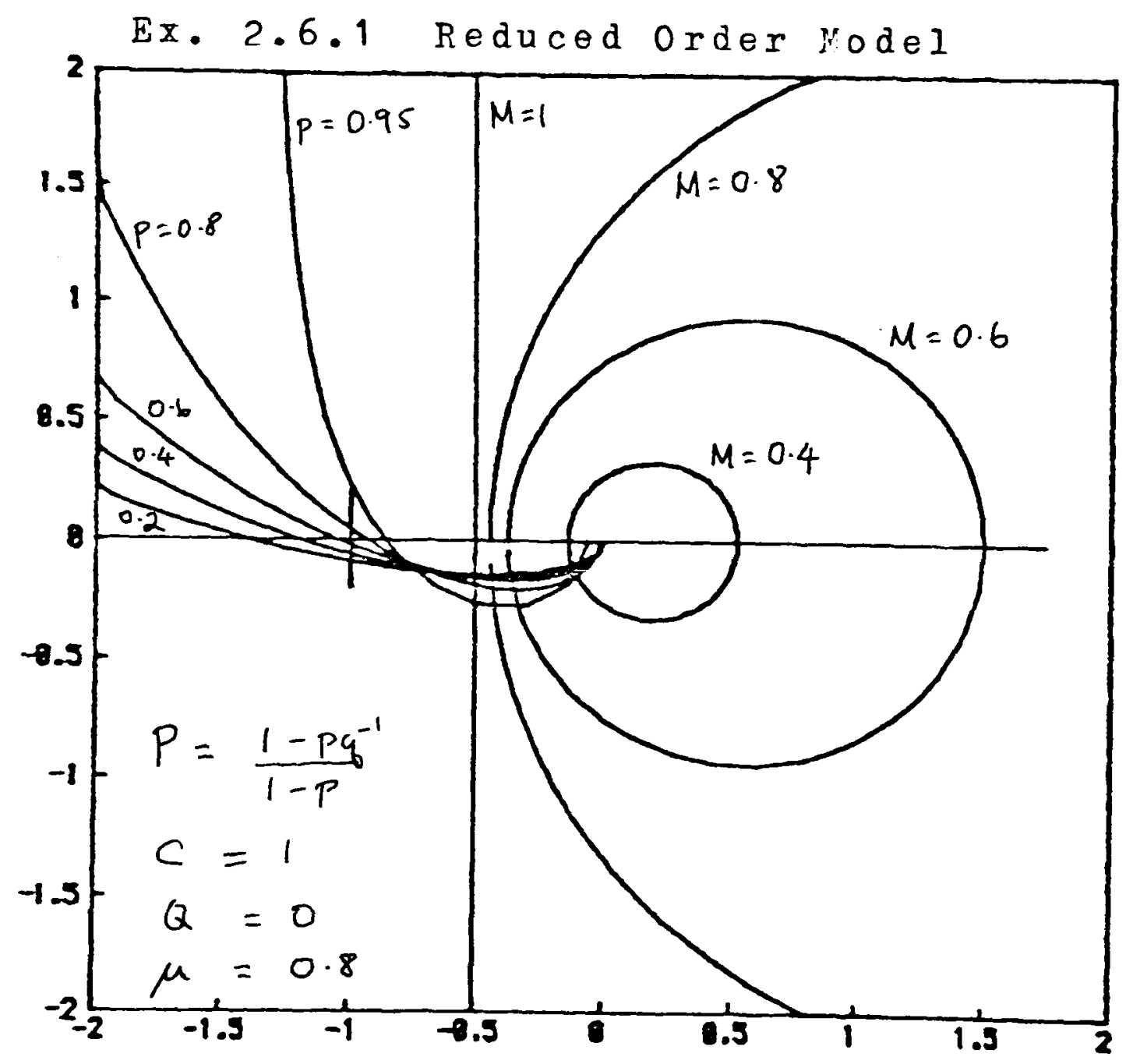

Fig. 2-16 Nyquist Loci for Varying $P$ 
ROBUSTNF.SS OF THF. NONADAPTIVF. CONTROTI.F.R

For $\mu=0.8$, Fig. 2-16 shows the Nyquist locus of $H\left(q^{-1}\right)$ for $P\left(q^{-1}\right)=\frac{1}{1-p}\left(1-p q^{-1}\right)$ and $p=0.2,0.4,0.6,0.8,0.95$. For $p \geqslant 0.8$, the Nyquist locus does not encircle the critical point. A slower model thus improves robust stability. Once $p$ is sufficiently large to ensure stability, "म̈ also decreases with increasing p. A slower $p$ also reduces the frequency range for which $|\overline{\mathrm{H}}|>1$ from

$$
\begin{aligned}
& 0.014 \omega_{s}<\omega<0.445 \omega_{s} \text { for } p=0.8 \text { to } \\
& 0.006 \omega_{s}<\omega<0.409 \omega_{s} \text { for } p=0.95
\end{aligned}
$$

There is also a frequency band for which $\left|(I+H)^{-1}\right|<I$ and this too is enlarged by a slower $\mathbf{P}$.

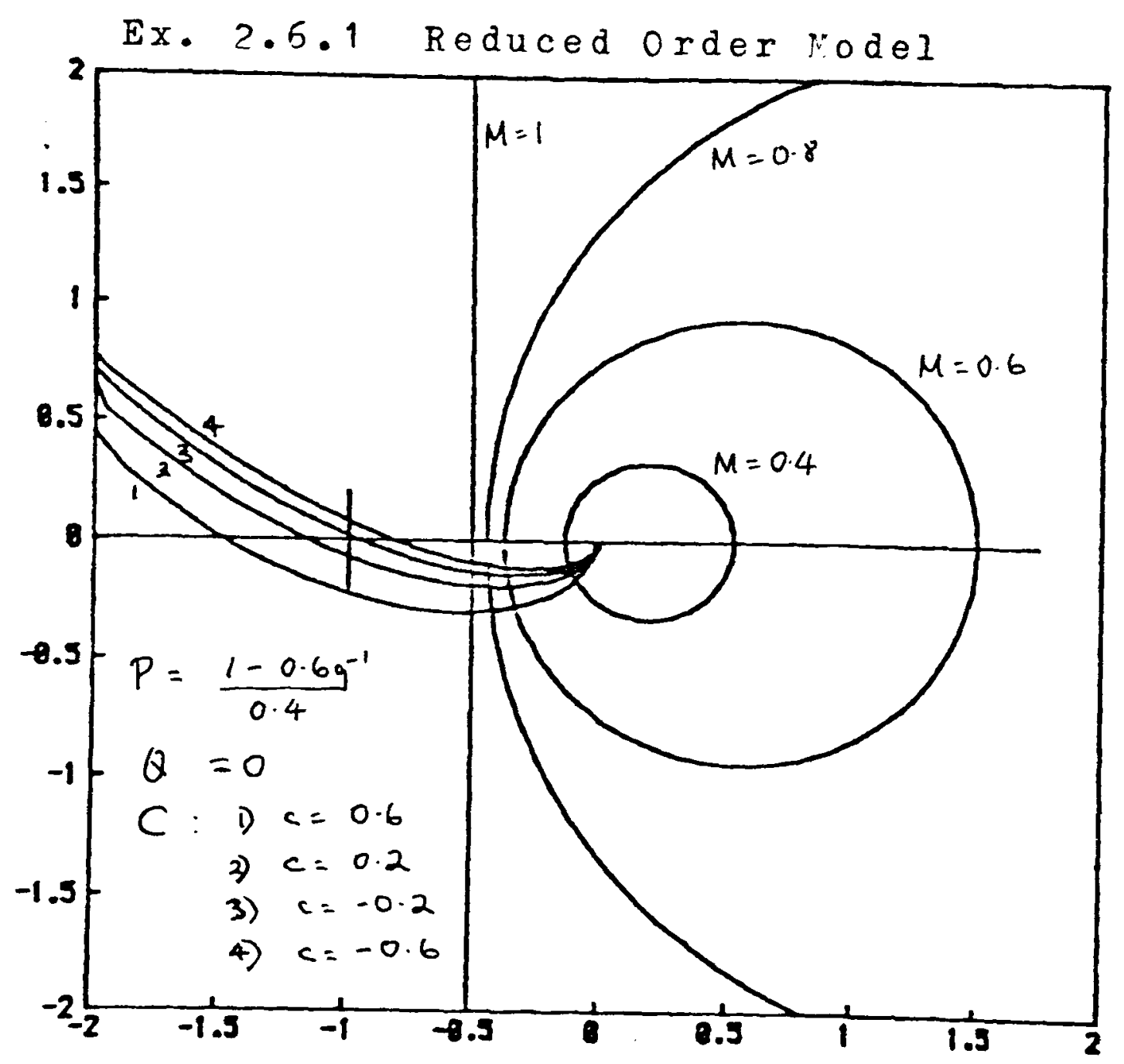

Fig. 2-17 Nyquist Loci for Varying C 
ROBUSTNF.SS OF THF. NONADAPTIVF, CONTROITIF.R

Alternatively, if we desire a faster $P$, eg. $p=0.6$, the observer polynomial, $c\left(q^{-1}\right)$ can be used to improve robust stability. Iet $c\left(q^{-1}\right)=\frac{1}{1-c}\left(1-c q^{-1}\right)$. Fig. $2-17$ shows the Nyquist plots of $B$ for $c=0.6,0.2,-0.2,-0.6$. Stability is ensured by $-c>0.2$. However, the range of frequencies for which $\left|(1+H)^{-1}\right|<1$ decreases from

$$
\begin{aligned}
& 0.056 \omega_{\mathrm{g}}<\omega<.944 \omega_{\mathrm{g}} \text { for } c=-0.2 \text { to } \\
& 0.072 \omega_{\mathrm{g}}<\omega<0.928 \omega_{\mathrm{g}} \text { for } c=-0.8
\end{aligned}
$$

Thus a slower predictor or observer improves robust stability but not disturbance attenuation.

A second alternative to ensure robust stability is to set $Q=\lambda$, a scalar weighting on the control law. As before, we consider $\mu=0.8$ and $p=0.6$. Fig. $2-18$ shows the Nyquist plots and there is no encirclement for $\lambda>0.3$. As $\lambda$ increases, the range of frequencies for which $\left|(I+H)^{-1}\right|<I$ also increases and $\|(I+H)^{-1}$ "decreases. Hence increasing $\lambda$ is good for robust stability and robust disturbance attenuation.

\subsubsection{Nonlinear Example}

Consider a nonlinearity at the output or input of a simple first order nominal system, $\frac{q^{-1}\left(1+0.5 q^{-1}\right)}{1-0.7 q^{-1}}$

Let $P=\frac{1}{1-p}\left(1-p q^{-1}\right)$

where $\mathrm{p}=0,0.2,0.4,0.6,0.8,0.9$ and $\mathrm{C}=1, Q=0$.

Fig. 2-19 shows the Nyquist plots of $q^{-1} \frac{\mathrm{F}}{\mathrm{PC}}$. Applying the circle criterion of Remark 5, Theorem 1, section 2.4.1, the closed loop system will be stable for any nonlinearity, $M_{i}$, such that $M_{i}-1$ is in the sector $(0, \alpha)$ where $\alpha$ depends on $p$ as shown in Table 1 . 
Ex. 2.6.1 Reduced Order Model

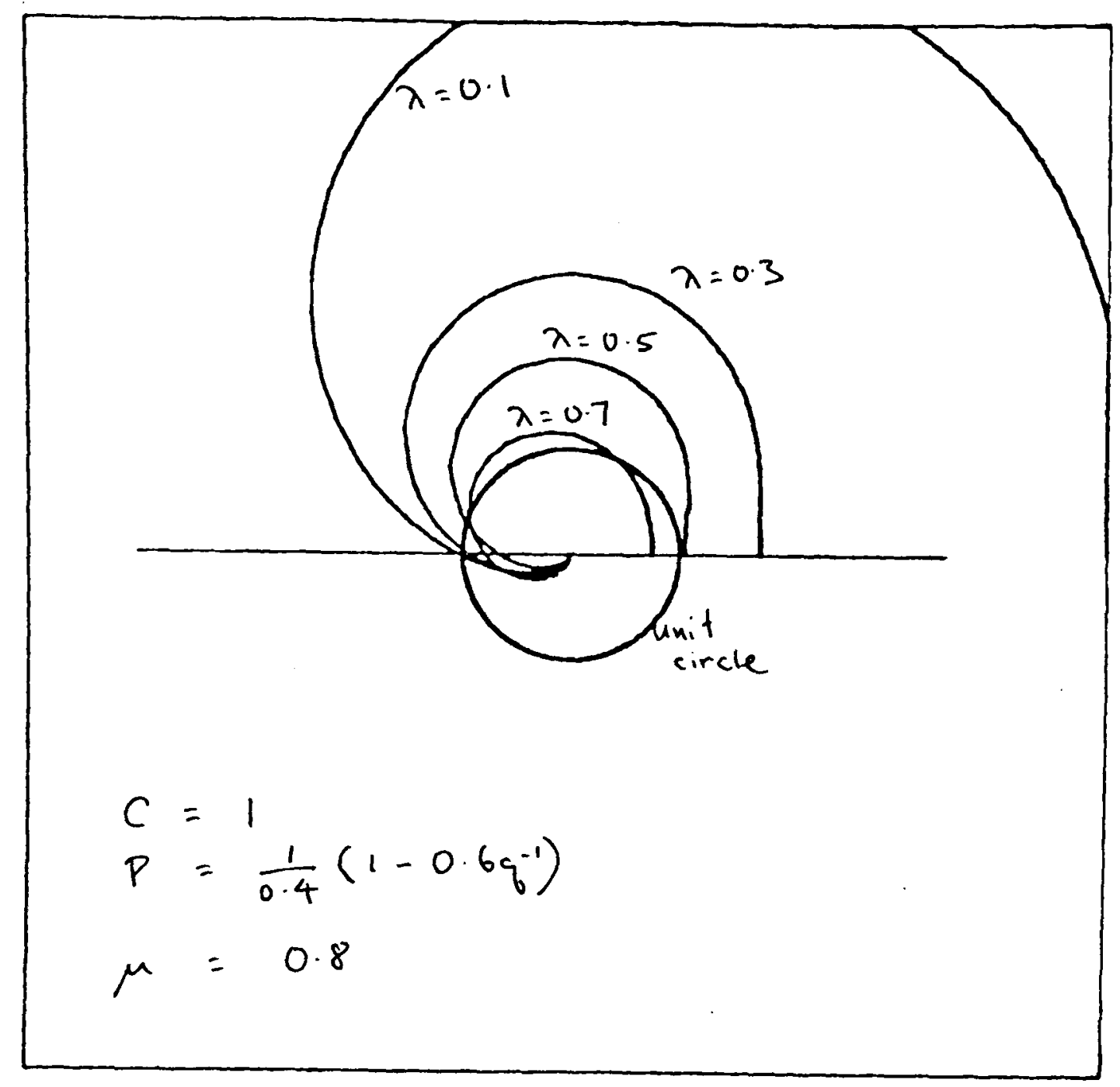

Fig. 2-18 Nyquist Loci for Varying $Q$

Ex. 2.6.2 Nonlinear Example

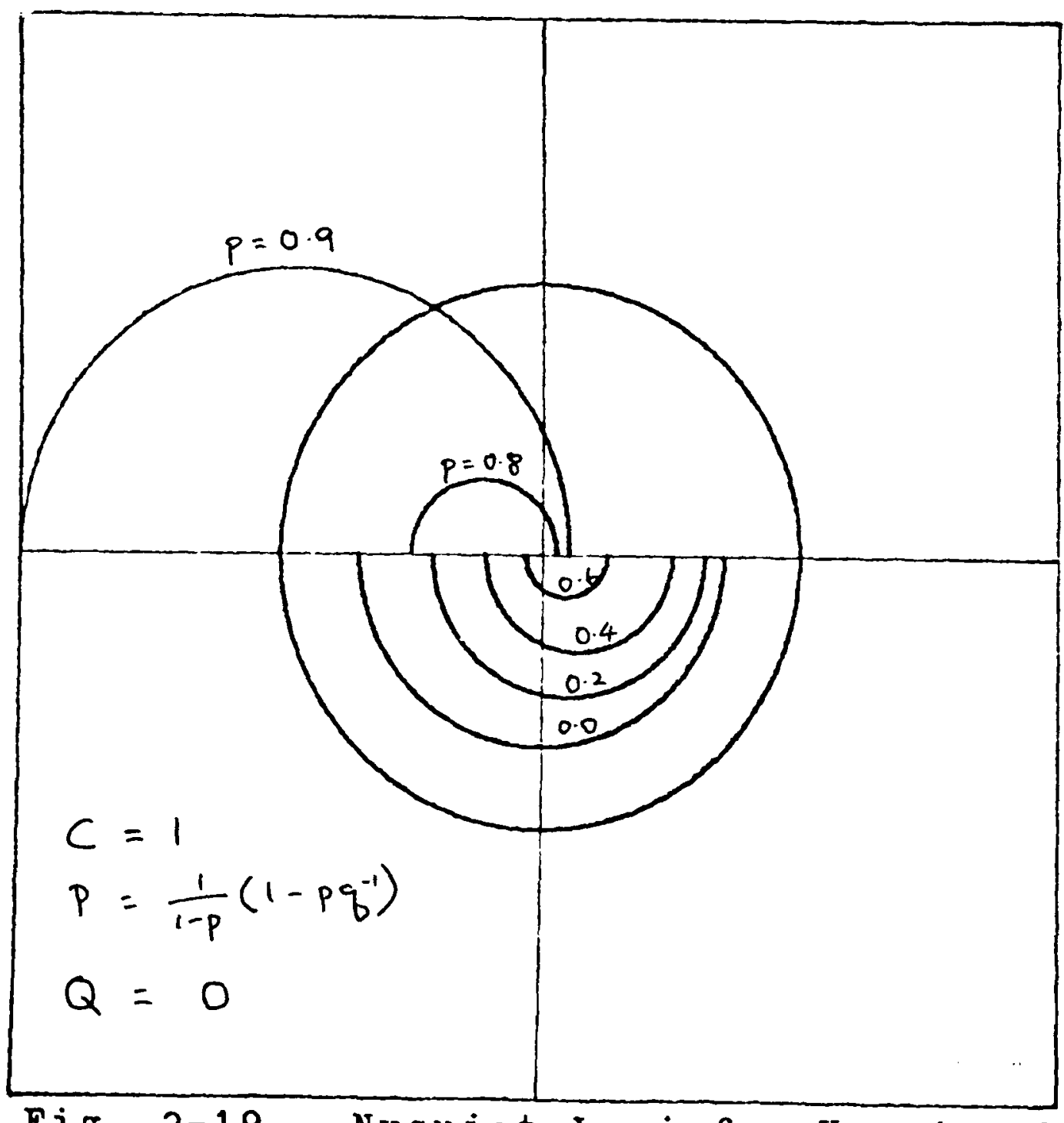

Fig. 2-19 Nyquist Loci for Varying 
ROBUSTNF.SS OF THF. NONADAPTIVF, CONTROIIIFR

\section{Table 1}

Varjation of upper sector bound with $P$

$\begin{array}{cl}\text { p } & \alpha \\ 0.0 & \\ 0.2 & 1.43 \\ 0.4 & 2.38 \\ 0.6 & 4.76 \\ 0.8 & 16.7 \\ 0.9 & 2.00 \\ & 0.50\end{array}$

As $p$ increases, the size of the robust stability sector increases until the desired model $\mathbf{p}^{-1}$ has a slower pole than the nominal plant, at which point the size decreases.

Theorem 1 of section 2.4 .6 can be used to show that the closed loop system will be unstable for $M_{i}^{-1}$ in the complementary sectors eg. for $p=0.4$, if $w_{1}-1$ is in the sector $(4.76, \infty)$, then there is an input $e(t)$ in $I_{2}$ which will lead to instability.

At the same time, for $p<0.7, \frac{F A}{P C}$ decreases as $p$ increases and from section 2.5 , this implies more robust disturbance attenuation. 
As in the linear case of section 2.6 .1 , careful choice of $c$ can also improve robustness. Let $c=\frac{1}{1-c}\left(1-c q^{-1}\right)$. Table 2 shows the robustness sectors for $M_{i}-1$ when $p=0.4$ and $c$ varieg from -0.9 to to.9.

\section{Table ?}

Variation of sector bounds with $C$

$\begin{array}{ccc}\mathbf{C} & \text { sector }\left[\mathrm{M}_{\mathbf{i}}-1\right] & \mathbf{F . A} \\ -0.9 & (0,0.09) & 12.1 \\ -0.5 & (0,0.70) & 2.43 \\ -0.2 & (0,1.72) & 1.52 \\ 0.2 & (0,8.33) & 1.12 \\ 0.5 & (0,3.85) & 1.26 \\ 0.9 & (0,0.25) & 5.8\end{array}$

The Choice of $c$ for robust stability thus appears to be more tricky than choice of $P$. As was the case with $P$, the pole should not be slower than the nominal plant pole as this leads to a marked deterioration.

Note that in the example so far, B does not come into consideration other than that it is assumed minimum phase. If $B$ is non-minimum phase, $P B+Q A$ must have no unstable roots.

I.et $B=1+1.5 q^{-1}, C=1$ and $Q=\lambda$. Let $P$ be as above i.e. $P=0.4$. PB+QA has no unstable roots for $\lambda>0.7$. Fig. 2-20 shows the Nyquist plots of $\frac{\bar{q}^{-k} \mathrm{FB}}{\mathrm{C}(\mathrm{PB}+Q \mathrm{QA})}$ for $\lambda=0.7$ to 3.0 . Applying the circle criterion again, the closed loop system is stable for $M_{i}-1$ in the sector $(0,4.33)$ when $\lambda=0.7$ and $(0,7.69)$ for $\lambda=3.0$. Hence increased control weighting improves robust stability. 
ROBUSTNF.SS OF THF. NONADAPTIVF: CONTROI.TIRR

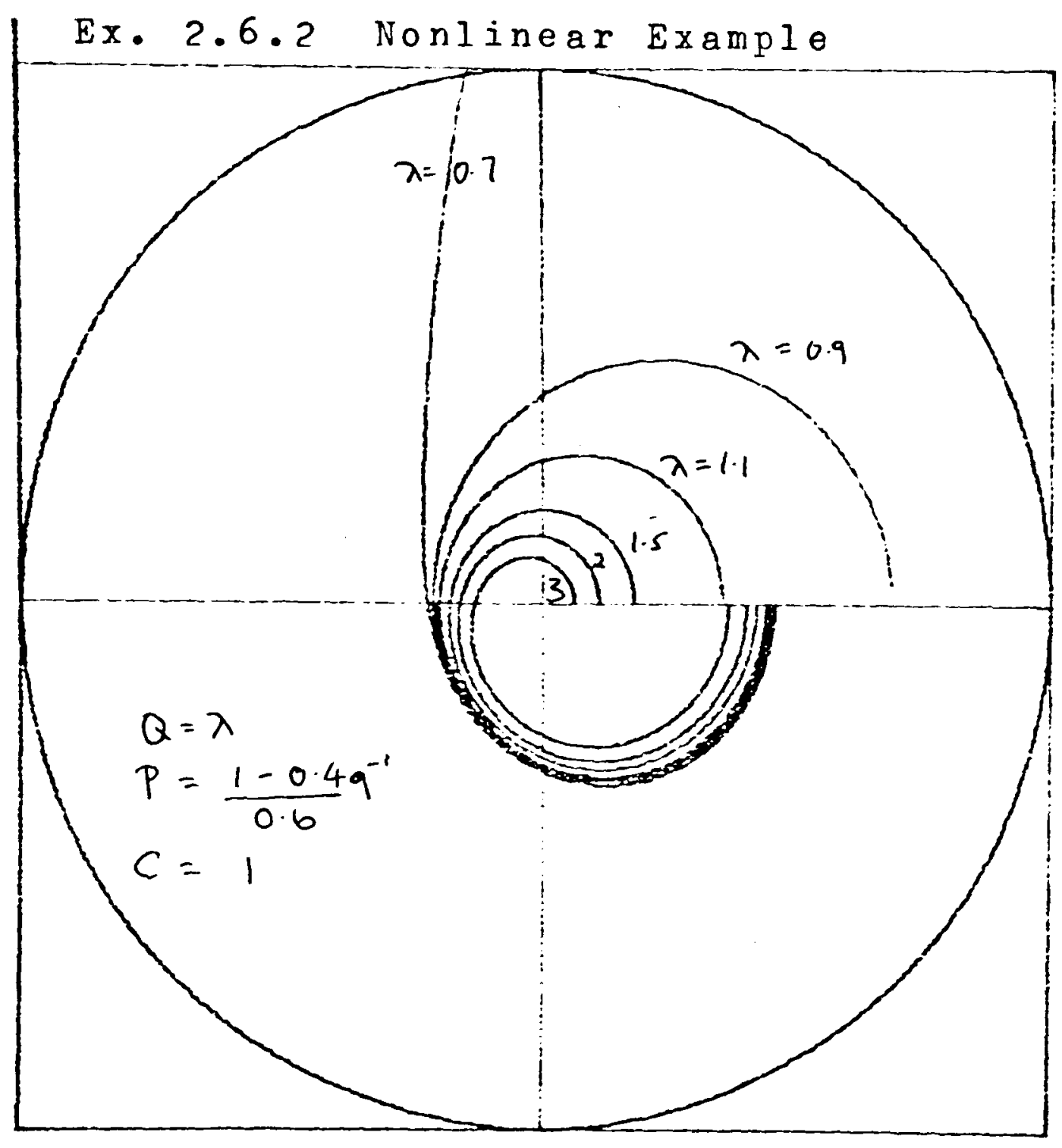

Fig. 2-20 Nyquist Loci for Varying $Q$ 
CHAPTER 3

ROBUSTNESS OF THF. ADAPTIVF, CONTROT,IF.R

\subsection{INTRODUCTION}

This chapter considers the robust stability of the adaptive self-tuner. In section 3.1 some properties of the matrix gain estimator used in the clarke-Gawthrop self-tuner are discussed. These properties characterige the estimator gain for both $1_{2}$ (i.e. finite energy) and $I_{\infty}$ (uniformly bounded) input sequences.

sections 3.2-3.4 are devoted to the clarke-Gawthrop form of the implicit self-tuning controller: the former considers the stability of the linear plant in the absence of plant perturbations while the latter introduces robustness considerations. Section 3.2 first reviews some ${ }_{2}$ stability results for the linear full order controller developed by Gawthrop and extends these results to uniform boundedness using the technique of exponential weighting. As the discussion in section 3.9 will suggest, this combination of $I_{2}$ and $I_{\infty}$ stability gives very strong results. Section 3.3 sets up the general exror feedback systems for the adaptive controllex in the presence of input and output plant perturbations. In section 3.4 we consider the robust stability of the implicit self-tuning controller under some simplifying assumptions: there is no control weighting $(Q=0)$ and the time delay is minimal 
ROBUSTNF.SS OF THF, ADAPTIVF: CONTROITIFR

$(k=3$.$) . Given a nonlinearity or a plant of larger order than that$ assumed for the nominal plant, conditions are, derived for the stability of the perturbed plant. The adaptation mechanism of the controller ensures that we need only require that a suitable nominal plant (linearised plant) exists for the closed loop system to be stable despite the plant perturbations. These results are also in terms of $1_{2}$ and $1_{\infty}$ stabijity.

Section 3.5 formalises an intuitively obvious instability counterpart of the preceding results for the implicit self-tuner. The instability results complement the stability results in that they only require the existence of an unstable nominal plant. Both stability and instability results may be conservative.

Some stability and robustness aspects of the explicit adaptive self-tuner are discussed in section 3.6. The section first explains why stability results for the explicit self-tuners have been so difficult to get. Fxplicit self-tuning controllers have the advantage that they can handle systems with time-varying delays easily. It has also been claimed that they are more robust to nonminimum phase systems. In this section, a simple robustness result for the explicit self-tuning controller is obtained. The results suggest that the explicit self-tuning controller may not be robust to nonminimum phase plants when the number of zeros is not accurately known.

The properties of the estimator in section 3.1 depend on a scalar sequence, $\sigma$. Although it is known that $\sigma(t)<1$, it has to be assumed that $\sigma_{0}=$ sup $_{t>t_{0}} \sigma<1$. Section 3.7 discusses this sequence. It turns out that for $\sigma_{0}$ to be small, the inputs and outputs to the plant must be 
ROBUSTNF,SS OF THF, ADAPTIVE, CONTROI,TF.R

'persistently exciting' in a well defined sense. In the feedback control context, it is not easy to ensure a priori that this is so.

One way of ensuring that the estimator has a priori testable properties i.e. $\sigma$ independence, is to use a scalar gain estimator. Section 3.8 discusses robust stability with such an estimator. The section shows that while neat theoretical results can be obtained with such an estimator, the results indicate that the controller is less robust than the matrix gain estimator. Section 3.9 concludes the chapter with a short discussion of some other robustness work.

\subsection{INPUT-OUTPUT PROPFRTIES OF THE FSTIMATOR}

In the previous chapter, no adaptation is used and hence the parameter error, $e_{p}(t)=0$ (cf Fqn. 2-33). In this section, a least squares type of estimator is considered, which is described by Eqns. 2-23 to 2-25, reproduced here for convenience.

$$
\begin{aligned}
& \hat{\theta}(t)=\hat{\theta}(t-1)+s^{-1}(t-k) x_{0}(t-k) \hat{e}_{1}(t) \\
& s(t)=\beta s(t-1)+x_{0}(t) x_{0}^{T}(t) \\
& s(0)=s_{0}>0 ; 0<\beta \leqslant 1
\end{aligned}
$$

As the subsequent analysis will utilise the tools of input-output analysis stability analysis, it is necessary to first describe the input-output properties of this estimator. The results of this section are essentially technical lemmas on the $1_{2}$ and $1_{\infty}$ gains of the functional relationships representing the estimator.

Define: $\dot{\theta}(t) \cong \hat{\theta}(t)-\theta$

where $\theta$ can be an arbitrary vector (of correct dimensions). We can 
ROBUSTNF.SS OF THF ADAPTIVE CONTROIJTIER

substitute Eqn. 4 in Fqn. 1 to get a parameter error update equation as

$$
\ddot{\theta}(t)=\dot{\theta}(t-1)+s^{-1}(t-k) x_{0}(t-k) \hat{e}_{1}(t)
$$

Define: $\quad e_{p}(t) \circ x_{0}{ }^{T}(t-k) \dot{\theta}(t-1)$ to be the output of the functional relationship, $H_{p}$ ' which has for input, $\hat{e}_{l}(t)$ (the estimated prediction error). ${ }_{p}$ can be used to represent the estimator in the error feedback systems we derive for the adaptively controlled system. Then in Gawthrop [40], it is shown that (see also proof of Jemma 2 in Appendix B.I)

$$
\begin{aligned}
& \sum\left[e_{p}{ }^{2}(t)+2 e_{p}(t) \hat{e}_{1}(t)+\sigma(t-k) \hat{e}_{1}{ }^{2}(t)\right] \\
& +\ddot{\theta}^{T}(k) s(0) \tilde{\theta}(k) \geqslant \ddot{\theta}^{T}(N) s(N-k) \tilde{\theta}(N), \quad \forall N>t_{0} \\
& \text { where } \sigma(t-k) \circ x_{0}^{T}(t-k) s^{-1}(t-k) x_{0}(t-k)
\end{aligned}
$$

The sequence $\sigma(t)$ will be examined in section 3.7. Here it suffices to note that

$$
\sigma(t)<1, \forall t>t_{0}
$$

and we assume that (cf section 3.7)

$$
\sigma_{0} \cong \sup _{t>t_{0}} \sigma(t)<1
$$

From Eqns, 6 and 2, it is possible, in principle, to select initial conditions $s(0)$ and $\dot{\theta}(k)$ such that

$$
\sum\left[e_{p}^{2}(t)+2 e_{p}(t) \hat{e}_{1}(t)+\sigma(t-k) \hat{e}_{1}{ }^{2}(t)\right] \geqslant 0
$$

From Eqn. 9 and the definition of conic operators in Chapter $1, \mathbf{B}_{\mathbf{p}}$ can be viewed as an $I_{2}$ operator exterior to the cone,

$$
\left\{-1-\left(1-\sigma_{0}\right)^{+1 / 2},-1+\left(1-\sigma_{0}\right)^{+1 / 2}\right\}
$$

I.emma I

$$
\text { Define: } \begin{aligned}
x(t) & \Leftrightarrow e_{p}(t)+\hat{e}_{1}(t) \\
& =e(t)-e_{m}(t) \quad \text { :from Eqn. } 2-33
\end{aligned}
$$

Let $B_{c p}$ denote the functional relationship between $x(t) \in 1_{2} e$ and 
ROBUSTNFSS OF THF, ADAPTIVE, CONTROITIFR

$\hat{e}_{1}(t) \in 1_{2} e^{\cdot}$ Then $\mathbf{r}_{c p}$ has a gain of $\left(1-\sigma_{0}\right)^{-1 / 2}$.

Proof

Eqn. 9 can be written as

$$
\begin{aligned}
& \sum\left[\left(e_{p}+\hat{e}_{1}\right)^{2}+(\sigma(t-k)-1) \hat{e}_{1}{ }^{2}\right] \geqslant 0 \\
& \text { or } \sum\left(e_{p}+\hat{e}_{1}\right)^{2} \geqslant \sum(1-\sigma(t-k)) \hat{e}_{1}{ }^{2} \\
& >\left(1-0_{0}\right) \sum^{N} \hat{e}_{1}{ }^{2}(t) \\
& \text { Hence } \sum^{N} \hat{e}_{1}{ }^{2}(t) \leqslant\left(1-\sigma_{0}\right)^{-1} \sum x^{2}(t)
\end{aligned}
$$

$\underline{\text { Remark }}$

If $x(t) \in 1_{2}$, i.e. the summations in the equations above exist in the limit as $N \rightarrow \infty$, then $\hat{e}_{1}(t) \in l_{2}$ and the gain of $H_{c p}$ is still $\left(1-\sigma_{0}\right)^{-1 / 2}$. Note also that by definition, $e_{p}(t)=B_{p}\left\{\hat{e}_{1}(t)\right\}$. Hence $B_{c p}$ can be written as $\left(1+H_{p}\right)^{-1} \cdot H_{c p}$ is an alternative representation for the estimator. It is useful where we wish to represent the estimator by a relationship which has bounded gain.

\section{Lemma 2 Fxponential weighting}

$$
\begin{aligned}
& \text { Define: } x_{1}(t) \leqq x(t) \exp (\alpha t)=\left(\hat{e}_{1}+e_{p}\right) \exp (\alpha t) \\
& \text { and } \quad e_{1}(t) \cong \hat{e}_{1}(t) \exp (\alpha t): \alpha \neq 0
\end{aligned}
$$

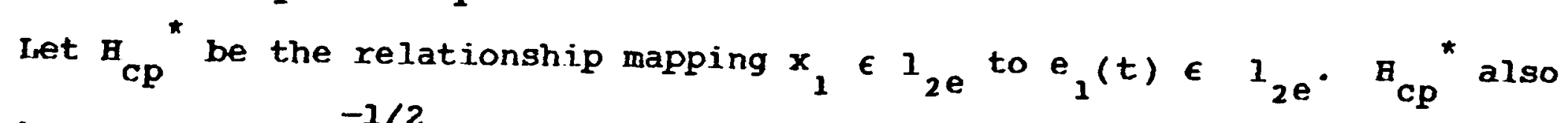
has gain $\left(1-\sigma_{0}\right)^{-1 / 2}$ i.e.

$$
\sum e_{1}^{2}(t)<\left(1-\sigma_{0}\right)^{-1} \sum_{1}^{N} x_{1}^{2}(t)
$$

if $1-\beta \exp (2 \alpha)>0$

\section{Proof}


ROBUSTNF.SS OF THF. ADAPTIVE CONTROIITFR

In Appendix B.1. If $\alpha=0.0$, then the proof of this lemma reduces to that for Iermma 1 .

Remark

If the forgetting factor, $\beta<1$, then there exists an $\alpha>0$ such that Eqn. Il is true. If $\alpha<0$, Fqn. 11 is always true for $0<\beta<1$.

$$
\begin{aligned}
& \text { If we write } \hat{e}_{1}(t)=H_{C p}\{x(t)\} \text {, then } \\
& \begin{aligned}
\hat{e}_{1}(t) \exp (\alpha t) & =\exp (\alpha t) H_{C p}\{\exp (-\alpha t) x(t) \exp (\alpha t)\} \\
& =\exp (\alpha t) H_{C p} \exp (-\alpha t) x_{1}(t)
\end{aligned}
\end{aligned}
$$

Bence $H_{\mathrm{Cp}}{ }^{\star}$ can be written as $\exp (\alpha t) \mathrm{H}_{\mathrm{Cp}} \exp (-\alpha t)$

${ }_{C P}{ }^{*}$ will be called the associate relationship to $\mathrm{H}_{\mathrm{CP}}$. I,emma 2 shows that the associate relationship has a finite gain in the space of $1_{2}$ sequences. In the next lemma, this will be used to show that the relationship ${ }^{B}{ }_{C p}$ ' is uniformly bounded i.e. if $x(t) \in I_{\infty}$, then so is $\hat{e}_{1}(t)$.

\section{Ieemma 3}

Assume that the initial states are selected so that Eqn. 9 holds for all $N>t_{0}$. Then

(i) if there exists $\alpha>0$ such that $I-\beta \exp (2 \alpha)>0$

(ii) $\sup _{t>t_{0}}|x(t)|<\infty$, i.e. $x(t) \in 1_{\infty}$

Then $\quad \sup _{t>t}\left|\hat{e}_{1}(t)\right|<\infty$

In fact, $\left|\hat{e}_{1}(t)\right|^{2}<\left(1-\sigma_{0}\right)^{-1}|x(t)|_{\infty}^{2}$

where the norm is the $I_{\infty}$ norm, defined as: $1 . \|_{\infty} \cong \sup _{t>t_{0}} 1.1$

Proof In Appendix $B .1$ 
ROBUSTNF.SS OF THE, ADAPTIVT, CONTROT,T,FR

\section{Remarks}

Temma 3 effectively says that if the relationship associated with ${ }_{C p^{\prime}}{ }^{B_{c p}}{ }^{*}$, maps $I_{2 e}$ into $I_{2 e^{\prime}}$ and if the input to $B_{c p} x(t)$ is uniformly bounded, then so is the output, $\hat{e}_{1}(t)$. Hence $H_{c p}$ has a finite $I_{\infty} g a i n$ given by Eqn. 14. I,emma 3 will be used later to deduce $1_{\infty}$ stability, given $1_{2}$ stability of an associated, exponentially weighted system.

Condition (i) is interesting in that it requires that for ${ }_{\text {cp }}$ to have a finite $I_{\infty}$ gain, the forgetting factor $\beta$ is strictly less than 1. when $\beta<1$, F.gn. 6 above is a strict inequality. Following willems[89], we can define:

A storage function: $v(t) \cong \dot{\theta}^{-T}(t) s(t-k) \dot{\theta}(t)$

and a supply rate: $e_{p}^{2}+2 e_{p_{1}} \hat{e}_{1}+\sigma(t-k) \hat{e}_{1}^{2}$ such that Eqn. 6 forms a dissipation inequality. The equality holds when the forgetting factor, $B=1$. If the storage function $v(t)$ is interpreted as energy stored in a system and the supply rate is interpreted as rate of energy input to the system, then Eqn. 6 with $\beta<1$ implies that the estimator is a dissipative system i.e. energy stored at time $N$ is always less than the sum of injtial stored energy, $\dot{\theta}^{-T}(k) S(0) \ddot{\theta}(k)$, and energy supplied till time $N$ (the left hand sjde of Eqn. 9).

The estimator equations of (1-5) can also be written in terms of the a posteriori prediction error, $\hat{e}_{0}(t)$. This has previously been used by Landau[58], Solo[79] and Gawthrop[41]. Denote the corresponding error quantities by suffix $0^{\prime}$ where

$$
\begin{aligned}
& \hat{\boldsymbol{e}}_{0}(t) \triangleq \Phi(t)-x_{0}{ }^{T}(t-k) \hat{\theta}(t) \\
& e_{p}{ }^{0}(t) \triangleq x_{0}{ }^{T}(t-k) \dot{\theta}(t)
\end{aligned}
$$

Let $\mathrm{H}_{\mathrm{cp}}{ }^{0} \operatorname{map}\left(\mathrm{e}_{p}^{0}+\hat{\mathrm{e}}_{0}\right)$ to $\hat{\mathrm{e}}_{0^{\circ}}$ Lemma 4 describes some properties of 
ROBUSTNESS OF THF, ADAPTIVF, CONTROII,FR

these a posteriori quantitieg.

I.emma 4

$$
\begin{aligned}
& \text { (i) } \hat{e}_{0}(t)=[1-\sigma(t-k)] \hat{e}_{1}(t) \\
& \text { (ii) } \hat{e}_{0}+e_{p}^{0}=\hat{e}_{1}+e_{p} \\
& \text { (iii) } \sum_{0}^{N} \hat{e}_{0}^{2}<\sum\left(e_{p}^{0}+\hat{e}_{0}\right)^{2} \\
& \text { i.e. gain of }{ }_{c p}{ }^{0}=1
\end{aligned}
$$

Proof In Appendix B.I

\section{$\underline{\text { Remarks }}$}

The advantage of using the a posteriori error quantities is that the gain of ${ }^{{ }_{C P}}{ }^{0}$ is independent of the sequence $\sigma$. Note that Lemma 4 describes exactly the same estimator as Lemma 1 does in terms of a priori quantities. Iemma 4 is useful in studying the stability of the linear full order controller which will be examined in the next section. It will be seen that Lemma 4 is not useful for the robustness question because a causal feedback control law cannot be formulated in terms of the a posteriori prediction error.

\subsection{STABILITY OF IINF.AR FUI,I, ORDER CONTROT,T,F,R}

In this section, some results for the $l_{2}$ stability of the closed loop system under self-tuning control, first presented by Gawthrop[38],[40],[41] are reviewed and extended to include $1_{\infty}$ stability. It is shown that if no attempt is made to estimate the noise parameters, stability depends only on the input-output properties of the estimator described in section 3.1 above. When noise parameters are estimated (c 
estimated) the results are more complicated and stability depends on the 'true' noise parameters, $c$, as well as on the estimator.

The 'true' plant is assumed to be linear. The order of the linear ARMA model which describes the plant, and delay, $k$, are also assumed to be known exactly. Hence in the system model of Fig. $2-2, M_{1}=M_{2}=1$ and the plant equation is

$$
\left.A\left(q^{-1}\right) Y(t)=B\left(q^{-1}\right) u(t-k)+C \delta q^{-1}\right) d(t)
$$

\subsubsection{No CoFstimation (Fixed observer)}

In this subsection, $c$, as in Chapter 2 , is taken to be a design polynomial and thus known. $\boldsymbol{M}_{i}=1$ implieg that the modelling error, $e_{m}(t)=0$ (from F.qn. 2-32). The control algorithm used is that described by Eqns. 2-21 to 2-27, where $y_{0}(t)=y(t)$ and $u_{0}(t)=u(t)$. The dimensions of the data vector, $x$ and the parameter vector, $\theta$ are sejected as in Eqns. 2-16 to 2-17 i.e $n_{G}=\operatorname{deg}\{B E\}$ and $n_{F}=\operatorname{deg}\{F\}$ where $\operatorname{deg}\{E\}=k-1:$ assumed known $\operatorname{deg}\{A\}, \operatorname{deg}\{B\}$ also assumed known

$$
\begin{aligned}
\operatorname{deg}\{F\} & =\operatorname{deg}\{A\}-1 \text { if } \operatorname{deg}\{C\}+\operatorname{deg}\{P\} \leqslant k-1+\operatorname{deg}\{A\} \\
& =\operatorname{deg}\{C\}+\operatorname{deg}\{P\}-k \operatorname{if} \operatorname{deg}\{C\}+\operatorname{deg}\{P\}>k-1+\operatorname{deg}\{A\}
\end{aligned}
$$

From the relationship between the exror quantities, Eqn. 2-33 and section 3.1, we can represent the closed loop system under self tuning control as in Fig. 3-1 below.

Using Lemma 1 of Section 3.1 , Fig. 3-1 reduces to Fig. 3-2 below, where $\mathrm{H}_{\mathrm{cp}}$ has an $1_{2}$ gain of $\left(1-\sigma_{0}\right)^{-1 / 2}$. Hence assuming that $\sigma_{0}<1$, $\hat{e}_{1} \epsilon I_{2}$ if $e(t) \epsilon l_{2}$ i.e. a finite energy disturbance results in a finite energy prediction error. If the disturbance, $d(t)$ is only 


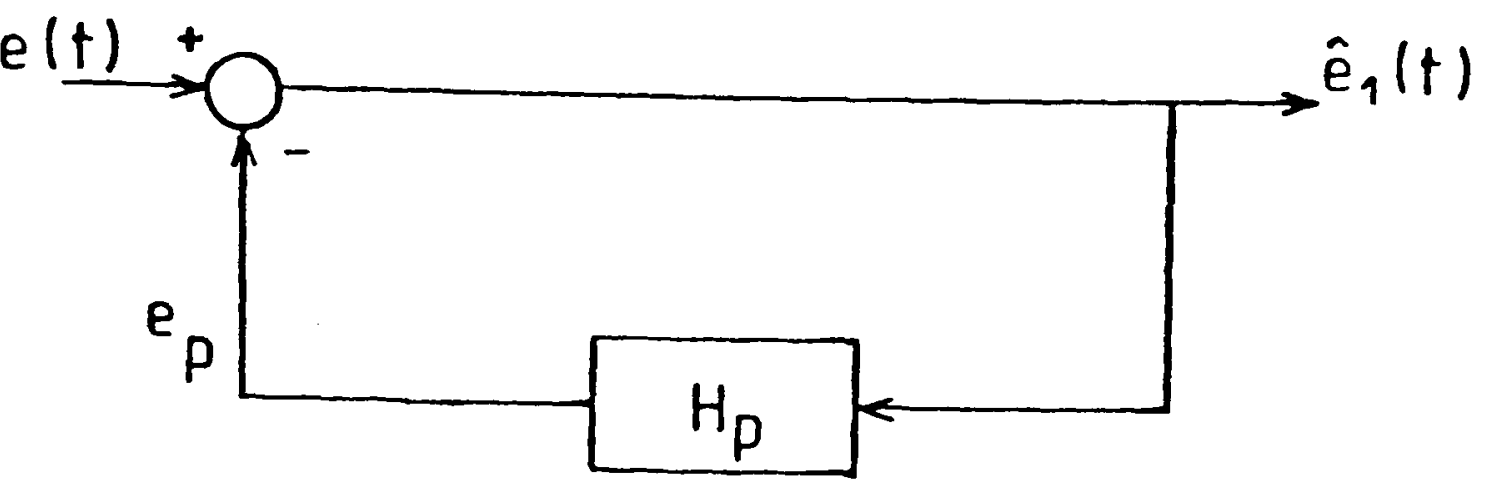

Fig. 3-1 Error Feedback system for Fixed

observer case (no c estimation)

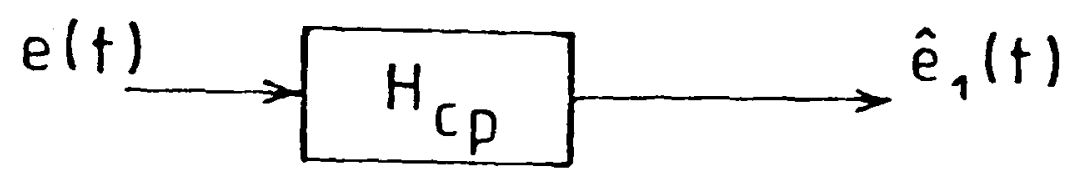

Fig. 3-2 Equivalent Relationship for Fixed observer case

uniformly bounded and is not necessarily of finite energy, then I,emma 3 of section 3.1 shows that the prediction error, $\hat{e}_{1}(t)$ is also uniformly bounded if the forgetting factor $\beta<1$.

From Eqns. 2-34 and 2-36 we get

$$
\begin{aligned}
& y(t)=\frac{B}{P B+Q A}\left[\hat{e}_{1}(t)+R w(t-1)\right]+\frac{C Q}{P B+Q A} d(t) \\
& u(t-1)=\frac{A}{P B+Q A}\left[\hat{e}_{1}(t)+R w(t-1)\right]-\frac{C P}{P B+Q A} d(t)
\end{aligned}
$$

where $\mathbf{a}=\tilde{y}=0$ and delay $k$ is assumed to be 1 .

Bence if $\hat{e}_{l}(t)$ is uniformly bounded or is finite energy stable, then $s o$ is $Y, u$ provided $[P B+Q A]^{-1}$ has stable poles. As in the non-adaptive case, if the plant is minimum phase, then $Q$ can be zero. If the roots of B are outside the stability region, then Eqn. 22 indicates that the control law is unstable if $Q=0$, even though the prediction error, $\hat{e}_{1}(t)$ is bounded. 
ROBUSTNF.SS OF THE ADAPTIVF, CONTROT,T,F.R

\subsubsection{Cosstimated}

In standard self-tuning theory, it is commonly assumed that $d(t)$ in F.gn. 20 is a zero mean white noise sequence and that $\frac{C}{A}(t)$ represents a djsturbance that is stationary and has a rational spectral density. In this subsection, $c_{0}$ is not a design polynomial, but is a polynomial in $q^{-1}$ with unknown coeffjcjents but of known order. The data vector can stil] be filtered by a known desjgn polynomjal, $\hat{c}^{-1}$.

If the parameters of the system are known (i.e. the coefficients of $A, B, C d$, then the steady state least squares predictor of $\Phi(t)=P y(t)$ is given by

$$
C \Phi^{*}(t+k / t)=F y(t)+G u(t)
$$

where $F$, and $E$ are obtained from the solution of the 'self-tuning identity', Eqn. 2-1.4, with $G=$ BF.

If a data vector filter, $\hat{C}^{-1}$ is added, we get from Eqn. 23

$$
\Phi^{*}(t+k / t)=F / \hat{C y}(t)+G / \hat{C u}(t)+\left(1-c_{b} \hat{C}\right) \Phi^{*}(t+k / t)
$$

Eqn. 24 can be written as

$$
\Phi^{*}(t+k / t)=x_{e}^{T}(t) \theta_{e}(t)
$$

where $x_{e}^{T}(t)=\hat{C}^{-1}\left[y(t), y(t-1), \ldots, y\left(t-n_{F}\right) ; u(t), \ldots, u\left(t-n_{G}\right)\right.$;

$$
\left.\Phi^{\star}(t+k-1 / t-1), \ldots .\right]
$$

$$
\theta_{e}^{T}(t)=\left[f_{0}, f_{1}, \ldots ; g_{0}, g_{1}, \ldots ; \bar{c}_{1}, \bar{c}_{2}, \ldots\right]
$$

where $\vec{c}_{i}=\hat{c}_{i}-c_{i i}$

When the parameters are unknown, $\theta_{e}$ is replaced by its estimate $\hat{\theta}$ and $x_{e}$ by its estimate $\hat{x}_{e}$. It is natural to replace $\Phi^{*}$ in F.qn. 26 by $\hat{\Phi}_{m}$, where we define: $\hat{\Phi}_{m}(t)=\hat{X}_{e}^{T}(t-k) \hat{\theta}_{e}(t-m)$ 
ROBUSTNF.SS OF THF. ADAPTIVF, CONTROT,T.F.R

For the estimator to be realisable, $m \geqslant 0$. In the self-tuning control]ers of Astrom et a][8],[9] and clarke-Gawthrop[19],[20], $m=$ delay, $k$. An advantage of this choice is that with $Q=0$, the control law sets $\hat{\Phi}_{m}(t)=\hat{\Phi}_{k}(t)=0$. From F.qns. 23 to 28 , it is clear that this avoids the need to estimate $c_{i}$. However, this choice makes stability analysis more diffjcult. Another possibility which will be considered is $m=k-1$. (Note that with $m=k$ or $k-1$, the expected value of $x_{e}^{T}(t-k) e(t)$ is zero, a requirement for convergence analysis. see $[40],[41] \cdot]$

The various error quantities are defined as in Chaptex 2 , with $x_{e}$ replacing $x$ and $\theta_{e}$ replacing $\theta$. For the rest of this section, assume that $k=1$

Choice 1: $m=k=1$

$$
\begin{aligned}
& \hat{e}_{1}(t)=\Phi(t)-\hat{x}_{e}^{T}(t-k) \hat{\theta}_{e}(t-1) \\
& e_{p}(t)=\hat{x}_{e}^{T}(t-k) \bar{\theta}_{e}(t-1) \\
& \hat{\Phi}_{m}(t)=\hat{x}_{e}^{T}(t-k) \hat{\theta}_{e}(t-1)
\end{aligned}
$$

From Eqns. 30 to 32

$$
\begin{aligned}
\hat{e}_{1}(t)+e_{p}(t) & =\Phi(t)-\hat{x}_{e}^{T}(t-k) \theta_{e} \\
& =\Phi(t)-\Phi^{*}(t / t-k)+x_{e}{ }^{T}(t-k) \theta_{e}(t-1)-\hat{x}_{e}(t-k) \theta_{e}(t-1) \\
& =c_{\delta} \hat{C} e(t)+\left(1-c_{6} \hat{C} \hat{e}_{1}(t)\right.
\end{aligned}
$$

Eqn. 33, together with Iemma 1 of section 3.1 forms the closed loop system of Fig. 3-3 below.

In the next theorem sufficient conditions for the stability of the feedback system of Fig. 3-3 will be given. 


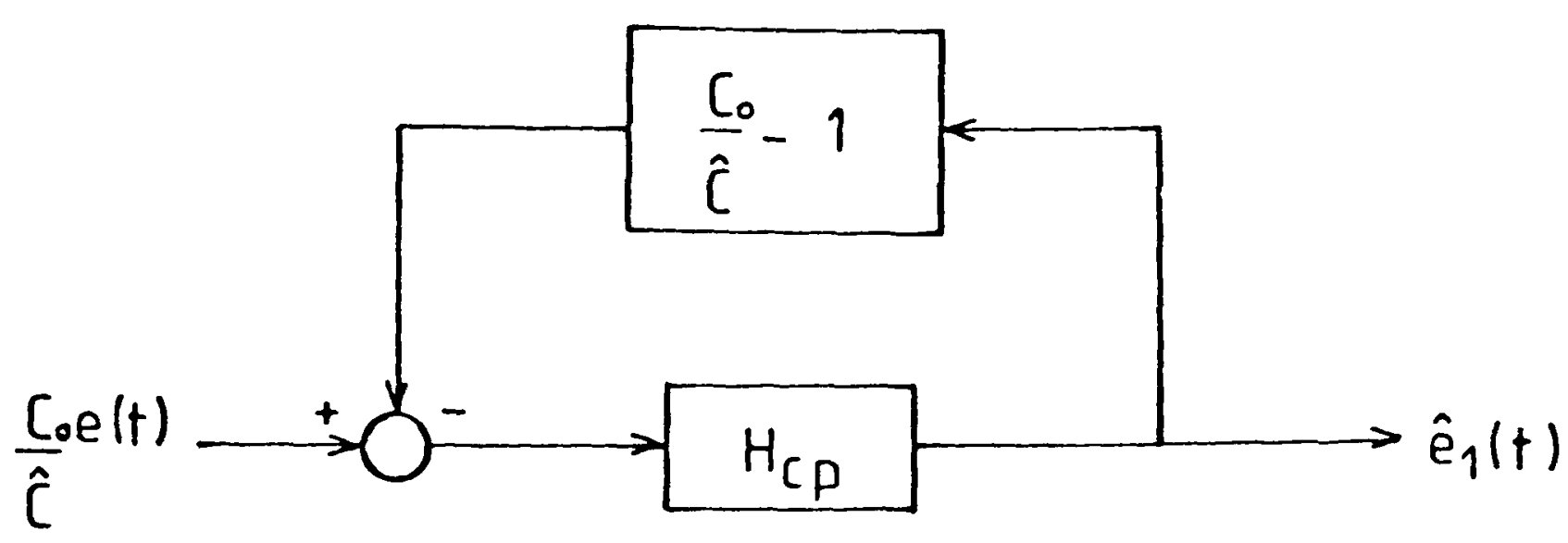

Fig. 3-3 Feedback system for C estimation algorithm (a priori errors)

\section{Theorem ].}

a) In the feedback system of Fig. $3-3$, if the input, $c / \hat{C}(t) \epsilon I_{2}$. i.e. is of finite energy, then the output, the estimated prediction error, $\hat{e}_{1}(t) \in I_{2}$ if

$$
\sup _{|q|=1}\left|\cos \hat{C}\left(q^{-1}\right)-1\right|<\left(1-\sigma_{0}\right)^{+1 / 2}
$$

i.e. the Nyquist locus of $c_{\gamma} \hat{C}-1$ lies within a critical circle of radius $\left(1-a_{0}\right)^{+1 / 2}$.

b) If in addition, there exists $\alpha>0$, such that

i) $1-\beta \exp (2 \alpha)>0$

ii) $\sup _{|q|=1}\left|\operatorname{co} \hat{C}\left([\exp (-\alpha) q]^{-1}\right)-1\right|<\left(1-\sigma_{0}\right)^{+1 / 2}$

i.e. the $\alpha$ shifted Nyquist locus lies within the critical circle, then the feedback system is also $I_{\infty}$ stable. In other words, the magnitude of $\hat{e}_{1}(t)$ is uniformly bounded if that of $c_{d} \hat{C} e(t)$ is.

Furthermore,

$$
\sup _{t>t_{0}}\left|\hat{e}_{1}(t)\right|<\gamma_{1} /(1-\gamma) \sup _{t>t_{0}}\left|\frac{C_{0}}{c} e\right|
$$

where $\gamma=\left(1-\sigma_{0}\right)^{-1 / 2}$. $\quad c_{\gamma} \hat{c}\left([\exp (-\alpha) q]^{-1}\right)-1 \|, \gamma_{1}=\left(1-\sigma_{0}\right)^{-\frac{1}{2}}$

\section{Proof}

a) follows from a direct application of the small gain theorem to Fig. 3-3.

b) In Appendix B.2 
ROBUSTNF.SS OF THF. ADAPTIVF, CONTROIITFR

口

\section{Remark I}

Theorem 1a) has already been presented in a slightly different form in Gawthrop[40]. Theorem 1b) appears to be new in a discrete time context and is an application of a long established idea due to Sandberg[76], Zames[94], Vidyasagar[83].

Theorem 1b) enables a frequency domain test for $1_{\infty}$ stability and hence makes application as simple as parseval's theorem does for 1 , stability. $I_{\infty}$ stability has previously been studied via a different method in Fgardt[33].

Remark 2

If $\sigma_{0}=0$, then the frequency domain conditions of the theorem may also be written as

$$
\operatorname{Re}\left\{\hat{C} / C_{D}-\frac{1}{2}\right\}>0 \quad \forall|q|=1
$$

If $\hat{C}=I$ and $c_{0}$ is first order, then this condition is always met. If $c_{0}$ has order greater than two, then a $\hat{C}$ filter will usually be needed, though the problem of choosing $\hat{C}$, given that $c_{0}$ is unknown remains.

\section{Remark 3}

As in section 3.2.1, (where $c_{0}$ is not estimated),

$$
Y(t)=\frac{B}{P B+Q A}\left[\hat{e}_{1}(t)+R w(t-1)\right]+\frac{C Q}{P B+Q A} \alpha(t)
$$

Boundedness of prediction error, $\hat{e}_{1}$ (in $l_{2}$ or $l_{\infty}$ sense) implies boundedness of $y(t)\left(I_{2} / I_{\infty}\right)$ if $[P B+Q A]^{-1}$ has stable poles. similarly, considering Eqn. 22, the control law is bounded if $[P B+Q A]^{-1}$ has stable 
ROBUSTNF.SS OF THF. ADAPTIVF, CONTROIITF.R

poles.

Choice 2: $m=k-1=0$

A disadvantage of Theorem 1 above is that the stability conditions depend on $\sigma_{0}$. In Iemma 4 of section $3.1, B_{c p}{ }^{0}$, the relationship which represents the estimator in terms of a posteriori error quantities is shown to have an $1_{2}$ gain of 1 , independent of $\sigma$. For the rest of this section, the $\Phi$ terms of Eqn. 26 above are approximated in the adaptive case by $\hat{\Phi}_{0}(t)=\hat{x}_{e}(t-k) \hat{\theta}_{e}(t)$.

$$
\begin{aligned}
\hat{e}_{0}(t)+e_{p_{0}}(t) & =\Phi(t)-\hat{x}_{e}^{T}(t-k) \hat{\theta}_{e}(t)+\hat{x}_{e}(t-k) \tilde{\theta}_{e}(t) \\
& =\Phi(t)-\hat{x}_{e}^{T}(t-k) \theta_{e} \\
& =\Phi(t)-x_{e}^{T}(t-k) \theta_{e}+\left[x_{e}^{T}(t-k)-\hat{x}_{e}^{T}(t-k)\right] \theta_{e} \\
& =e(t)+(1-\operatorname{co} \hat{C})\left(\hat{e}_{0}-e\right) \\
& =\operatorname{cod} \hat{C} e(t)-(c \phi \hat{C}-1) \hat{e}_{0}
\end{aligned}
$$

Fqn. 35 and Ierma 4 of section 3.1 together form the closed loop system of Fig. 3-4 below. Theorem 2 states sufficjent conditions for the stability of this closed loop system.

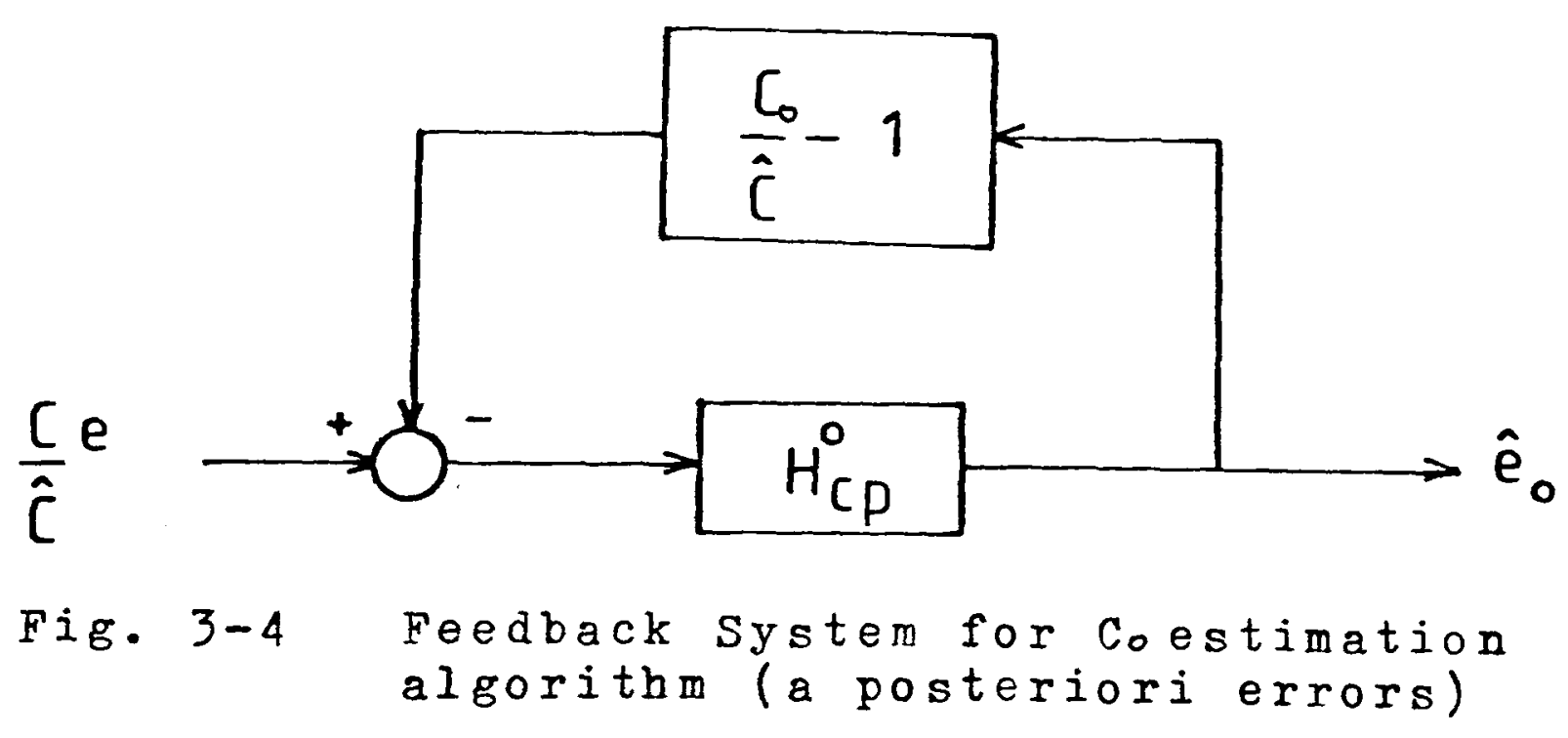

Theorem 2 
ROBUSTNF.SS OF THF ADAPTIVE, CONTROTITFR

If the conditions of Theorem 1 are satisfied, when applied with $\sigma_{0}=0$, then we have that $\hat{e}_{0}$ is bounded $\left(1_{2} / 1_{\infty}\right)$ if the input $c \hat{c} e(t)$ is bounded in the same $\left(1_{2} / 1_{\infty}\right)$ sense.

Proof

Follows from that of Theorem 1, but with I,emma 4 of Section 3.1 used instead of Isemma 3 .

$\square$

\section{Remarks}

Fig. 3-4 and F.qn. 35 describes a slightly different algorithm from that of Fig. 3-3. Note however, that Eqng. 21 to 22 relating the estimated prediction exror $\hat{e}_{1}$ to the output and input is still valid. since from I,emma 4 , Section $3.1, \hat{e}_{0}>\left[1-\sigma_{0}\right] \hat{e}_{1}, \hat{e}_{1}$ is bounded (in the appropriate sense) if $\hat{e}_{0}$ is bounded, assuming that $\sigma_{0}<1$. The effect of choosing $m=0$ is to move the $\sigma$ dependent terms out of the feedback loop of Fig. 3-4. Remark 2 of Theorem 1 is thus also valid here.

Both Theorems 1 and 2 are valid for the case of $k=1$ (minima) delay). The case of $k>1$ is deferred till Chapter 4.

In Chapter 1, a brief review has been made of the $1_{\infty}$ stability results of Fgardt[33] for a generalised self-tuner with a slightly different estimator (bilinear in the parameters; $b_{0}$. lst coefficient of $B\left(q^{-1}\right)$ is assumed known). Though the method of proof is different, an assumption similar to $\sigma_{0}<1$ had also to be made. The interesting point is that when a stochastic approximation estimator (cf section 3.8) is used, $\beta<1$ was required there too, to ensure that the estimator was NOT of monotonically decreasing gain. This was to guarantee that if the input or output sequences become unstable, the estimator has sufficient 
ROBUSTINESS OF THF. ADAPTIVF, CONTROI,T.F.R

gain to adjust the parameters quickly.

\subsection{F.RROR MODFL: ADAPTIVF. CASE}

In this section, we return to the robustness question. As in Chapter 2 and section 3.2.1, $\mathrm{C}$ is a design polynomial and there is no noise parameter estimation. The error feedback systems for the adaptively controlled plant will be derived, for plant perturbations at the output and at the input of the nominal plant.

In the adaptive case, the parameter error, $e_{p} \neq 0$. From Eqn. 2-33, we have the relationship between the error quantities as

$$
\hat{e}_{1}(t)+e_{p}(t)=e(t)-e_{m}(t)
$$

From Lemma 1 of section $3.1, H_{c p}$ represents the functional relationship between $\left(e-e_{m}\right)$ and $\hat{e}_{1}$ i.e.

$$
\hat{e}_{1}(t)=B_{c p}\left\{e-e_{m}\right\}
$$

Consider the output error case of $a=0$. From Eqn. 37, and Section 2.3.2, we get the error feedback model of Fig. 3-5 below.

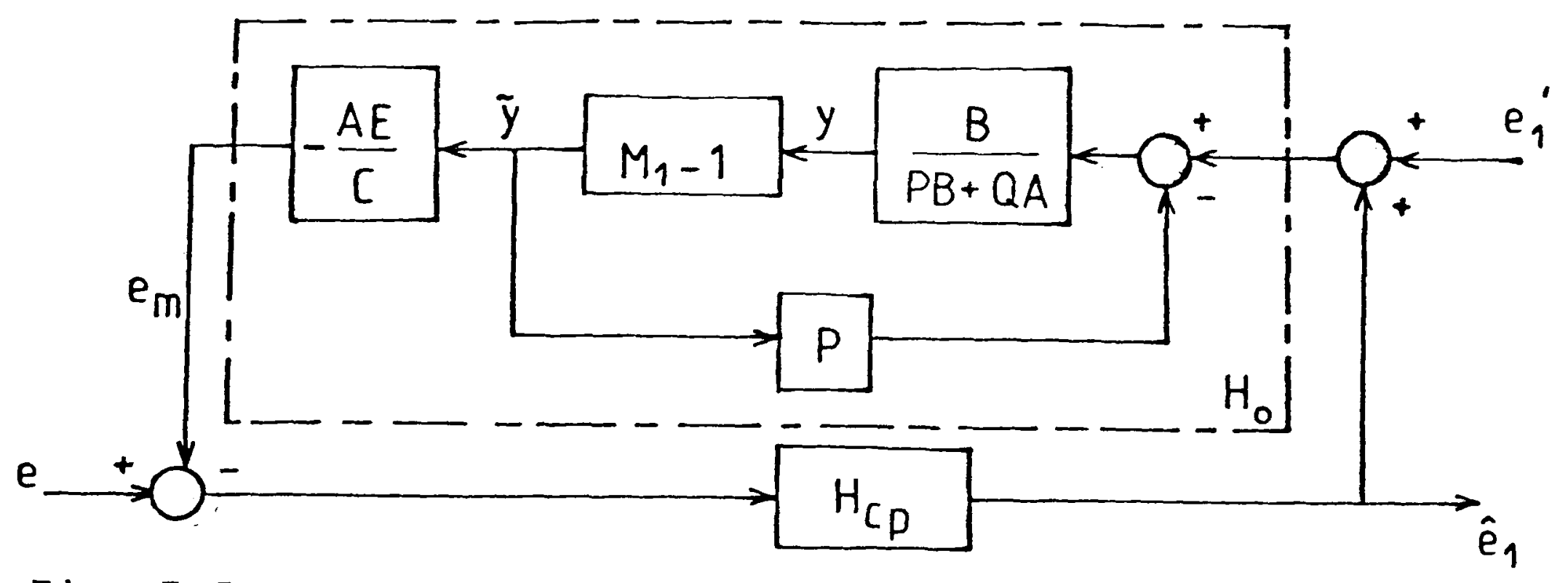

Fig. 3-5 General Feedback system for output Error case 
ROBUSTNF.SS OF THF, ADAPTIVF, CONTROI,TIF.R

Comparing with the non-adaptive case (Fig. 2-7), it is obvious that the only difference between the adaptive error model and the non-adaptive model is the nonlinear, time-varying relationship ${ }_{c p^{\prime}}$ which represents the presence of an estimator.

Similarly, the error feedback model in the adaptive case when $\$=0$ (input error only), derived from the error relationships of section 2.3.2 and the control law of Eqn. 2-26 is as shown in Fig. 3-6 below. (corresponds to Fig. 2-9: non-adaptive input error)

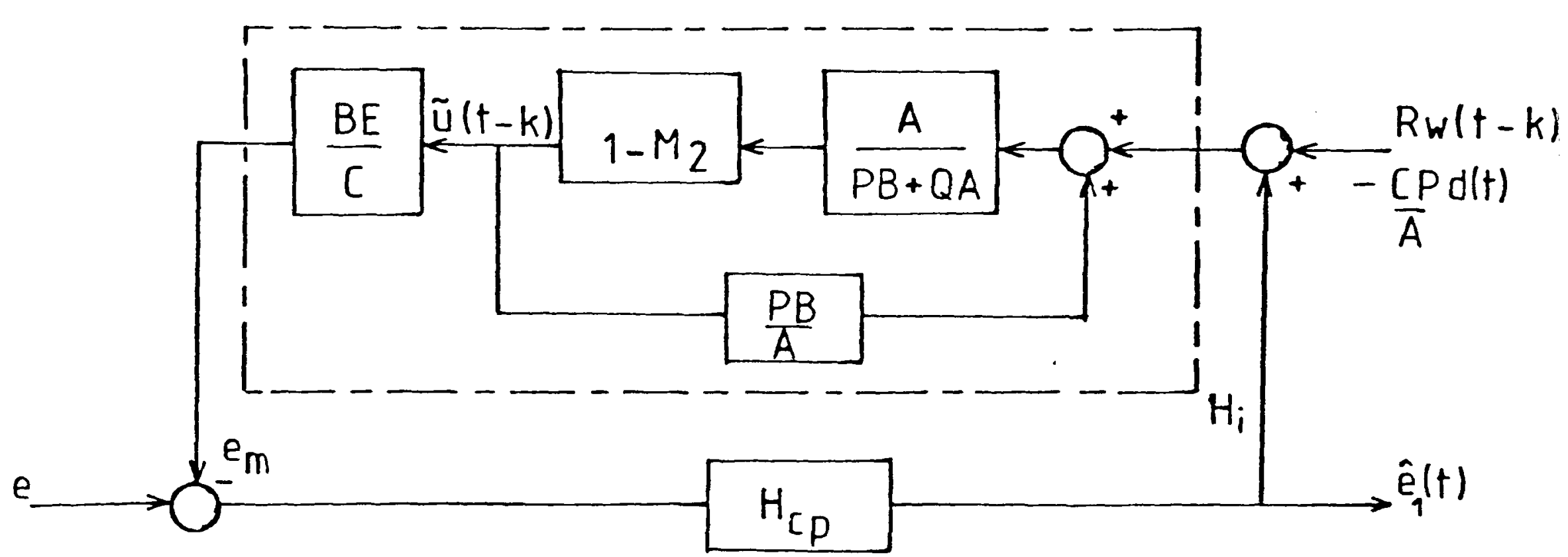

Fig. 3-6 General Feedback System for Input Error case

\subsection{ROBUST STABILITY OF ADAPTIVE CASE}

In this section, the robust stability of the adaptively controlled system is studied when the nominal plant is assumed to have minimal delay $(k=1)$ and the design polynomial $Q=0$.

The error feedback model for the output error case $(\alpha=0)$ (from Fig. 3-5) can be simplified to be that in Fig. 3-7.

In the input error case $(Y=0)$, we have, from Fig. 3-6, the error feedback system of Fig. 3-8 below. 
ROBUSTNF.SS OF THE ADAPTIVF. CONTROT,TF.R

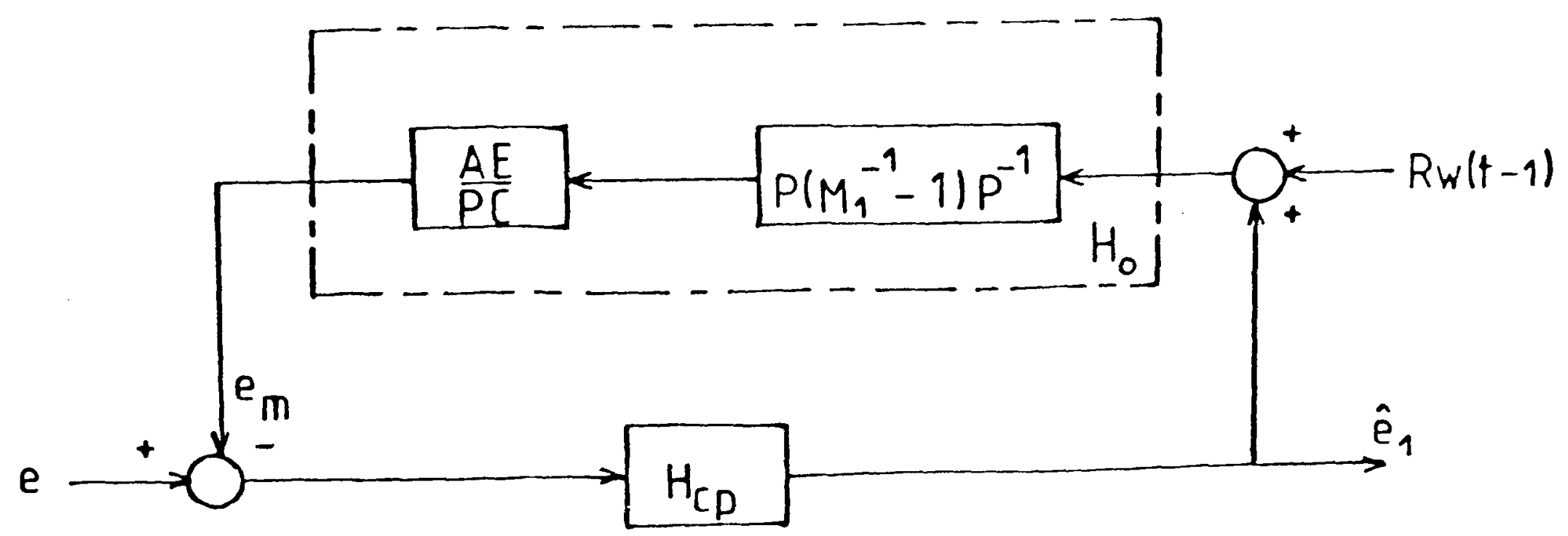

Fig. 3-7 Simplified Feedback System for Output Error

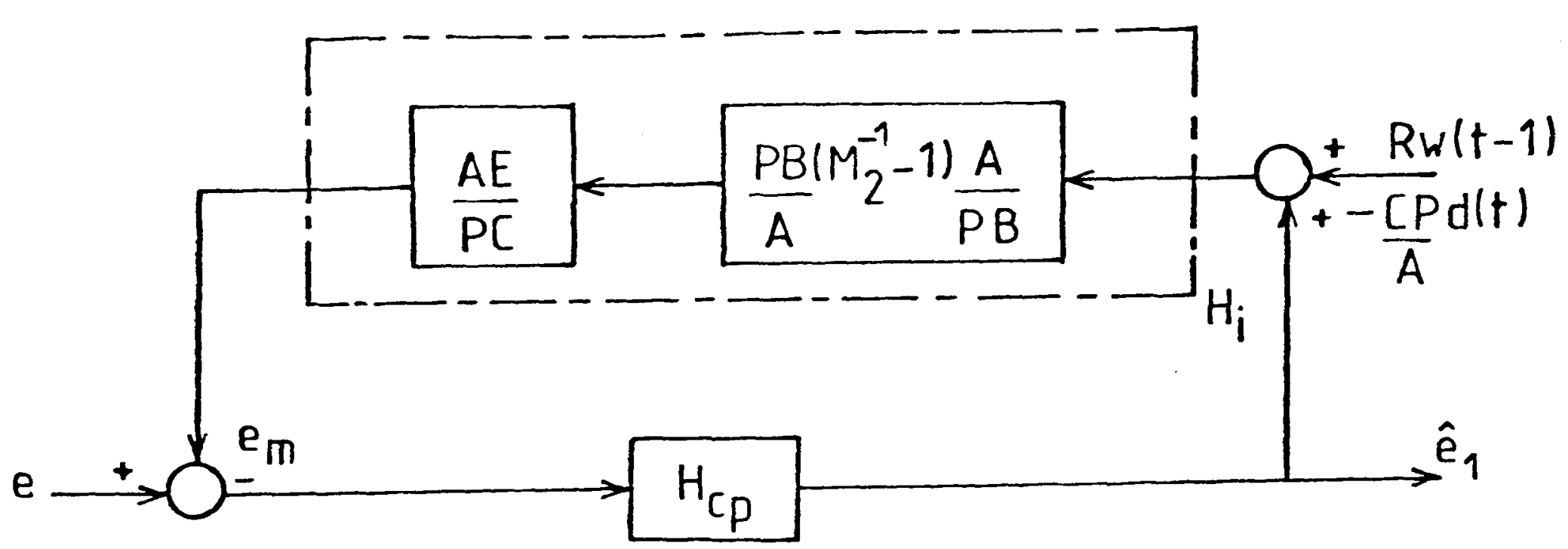

Fig. 3-8 Simplified Feedback system for Input Error

At this stage, it is important to note that the input-output properties of the estimator relationship, represented by $\mathrm{H}_{\mathrm{cp}}$ and discussed in section 3.1, are independent of the nominal parameter vector $\theta$. This fact will be seen to simplify the analysis considerably.

The error feedback models of Figs. 3-7 and 3-8 above correspond to the non-adaptive versions of Corollaries 2.1 and 2.2 in sections 2.4 .1 and 2.4.2. We consider first the output error case, $0=0$. 
ROBUSTNF.SS OF THF, ADAPTIVE CONTROIITFR

\subsubsection{Output F.r.ror $(a-0)$}

The error feedback system is shown j.n Fig. 3-7 above. When the 'true' system is linear, but is of higher order than assumed in the controller (dimension of parameter and data vectors selected to be smallex than what they should be according to section 3.2.1 inadvertently or otherwise), we have from section 2.2.1 that

$$
M^{-1}-1=\frac{B A}{A B} 0-1=\frac{B}{A}\left[\frac{A}{B} 0-\frac{A}{B}\right]
$$

and $H_{0}$ in Fig. $3-7=\frac{F}{P C B_{0}}\left(A_{0} B-B_{0} A\right)$

Thus, gain $\left\{\mathrm{B}_{0}\right\}=\sup _{|q|=1}\left|\frac{E}{P C B_{0}}\left(A_{0} B-B_{0} A\right)\right|$

I.et $H_{0}{ }^{*}$ be the associate operator that maps the exponentially weighted input and output sequences of $\mathrm{H}_{0}$

$$
\text { j.e. } e_{m}(t) \exp (\alpha t)=B_{0}^{*}\left\{\left[\hat{e}_{1}(t)+R w(t-1)\right] \exp (\alpha t)\right\}
$$

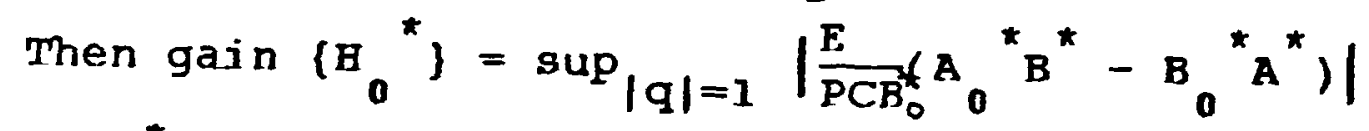

where $I^{*}\left(q^{-1}\right)=I\left([\exp (-\alpha) q]^{-1}\right)$

: from proof of Theorem $1(b)$ in section 3.2 .2

When $M_{1}$ is nonlinear, the gain of $\mathrm{H}_{0}$ can be separated into a linear and a nonlinear part.

$$
\operatorname{gain}\left\{\mathbf{F}_{0}\right\} \leqslant \sup _{|q|=1}\left|\frac{F A}{P C}\right| \operatorname{gain}\left(P\left(M_{1}^{-1}-1\right) P^{-1}\right\}
$$

Iemma $I$ below states a special case where $\operatorname{gain}\left(P\left(M_{1}^{-1}-1\right) P^{-1}\right\}$ is easily evaluated.

\section{Lemma 1}

Iset $L\left(q^{-1}\right)$ be a linear operator such that $L\left(q^{-1}\right)$ and $L^{-1}\left(q^{-1}\right)$ are stable. Assume that $M_{n}$ is a nonlinearity which is continuously Frechet differentiable over its domain and is such that $\operatorname{IM}_{n} I^{-1}\{0\}=0$.

Then $\operatorname{gain}\left(\operatorname{TH}_{n} L^{-1}\right)=\operatorname{ITM}_{n} L^{-1}$ 
ROBUSTNF.SS OF THF. ADAPTIVE, CONTROTIT.FR

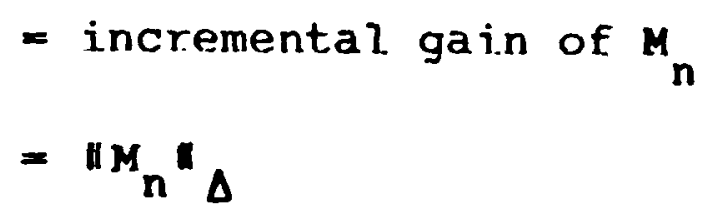

Proof In Appendix B.3

If $M_{1}^{-1}-1$ i.s time-invariant, memoryless and maps 0 into 0 , and it i.s al.so incrementa17y gain bounded, then the conditions of Tiemma 1 are met..

Rence gain $\left\{\boldsymbol{B}_{0}\right\} \leqslant \sup _{|q|=1}\left|\frac{F . A}{P C}\right| M_{1}{ }^{-1}-1 \|_{\Delta}$

When $M_{1}^{-1}-7$ i.s memoryless, i.t is obvious that the associated operator, mapping exponentially weighted sequences i.s identical to the operator itself and hence:

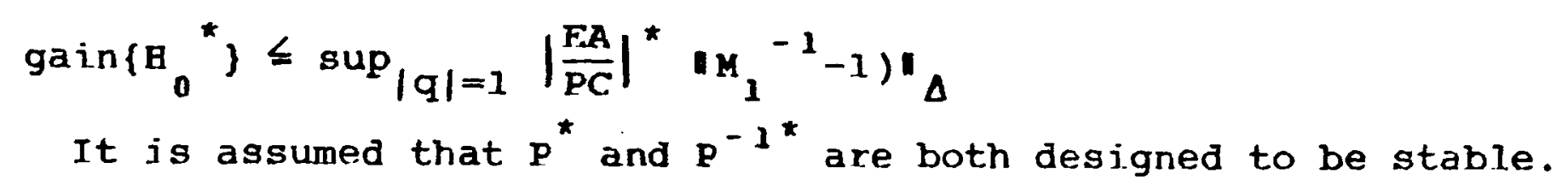

The next theorem states sufficent conditions for stability in this case.

\section{Theorem 1}

a) The system of Fig. 2-2 with $k=1$ and $\mathbf{a}=0$, controlied by the self-tuning adaptive controljer with $Q=0$ is stable in the $I_{2}$ input-output sense if the following conditions hold:

i) P, C, the design polynomials are selected to be strictly stable

ii) AND (linear higher order case)

$B_{0}$ is strictly stable

there exists arbitrary $A$ and stable $B$ such that gain $\left\{B_{0}\right\}$ in

Eqn. 40 is less than $\left(1-\sigma_{0}\right)^{+1 / 2}$

OR ( $M_{1}$ is a memoryless nonlinearity)

$B_{0}$ is strictly stable

there exists an arbitrary scalar, $\gamma \neq 0$, such that

$$
\operatorname{gain}\left\{\mathbf{R}_{0}\right\} \leq \sup _{|q|=1}\left|\frac{E A}{P C}\right| \cdot \gamma M_{1}^{-1}-1 \|_{\Delta}<\left(1-\sigma_{0}\right)^{+1 / 2}
$$


ROBUSTNF.SS OF THF. ADAPTIVF CONTROT.T.F.R

b) The same system is also $1_{\infty}$ stable if the conditions above are satjsfjed for the assocjated system $B_{0}^{\star}$ (with $P^{\star}, B_{0}^{*}, A_{0}^{*}$ etc), using an $\alpha>0$ such that $1-\beta \exp (2 \alpha)>0 . \beta$ is the forgetting factor of the estimator.

\section{Proof}

a) Consider Fig. 3-7. Assume that the setpoint, w and the disturbance, $a(t) \epsilon I_{2}$. Then if ${ }_{0}$ is a stable operator, the small gain theorem requires that for $\hat{e}_{1}(t) \in 1_{2}$,

$\operatorname{gajn}\left(\mathrm{H}_{0}\right)<\left(1-\sigma_{0}\right)^{+1 / 2}$

Condition i) follows from the requirement that ${ }_{D}$ be stable. So does requiring $B_{0}$ to be stable in the higher order case and stable $B$ in the nonjinear case.

In the linear higher order case, the arbitrariness of $A$ and $B$ arises from the fact that linear scalar operators commute i.e. the transfer function $\frac{B}{A} 0=\frac{B}{A} \frac{B}{A} 0 \frac{A}{B}$ for any $A, B$ and from the nondependence of $B$ upon the nominal parameter vector, $\theta$ and hence upon A, B.

In the nonlinear case, the cascaded operator, $N \frac{B}{A}$, can be written as $N \frac{1}{\gamma} \frac{\gamma B}{A}=N \frac{1}{\gamma} \frac{B^{\prime}}{A}$ where $\gamma$ is an arbitrary scalar.

From Eqns. 2-34 to 2-37, we can write

$$
\begin{aligned}
& y_{0}(t)=\frac{1}{P}\left[\hat{e}_{1}(t)+R w(t-k)\right] \\
& u(t-k)=\frac{A}{P B}\left[\hat{e}_{1}(t)+R w(t-k)-P Y(t)\right]-\frac{C}{B} d(t)
\end{aligned}
$$

Eqn. 48 shows that even if the prediction error is bounded, the control signal is not unless $B$ is strictly stable.

b) The proof parallels that of Theorem ib) in section 3.2 .2 and will only be outlined. In Fig. $3-7$, if $e(t)$ and $R w(t-1)$ are in $I_{\infty}$, then the 
exponentially weighted sequences, $e(t) \exp (\alpha t)$ and $R w(t-1) \exp (\alpha t)$ are in ${ }_{2} e^{\cdot}$ The small gain theorem applied to the associated system shows that $\hat{e}_{1}(t) \exp (\alpha t) \in 1_{2 e}$ and hence $\hat{e}_{1}(t) \in 1_{\infty}$.

\section{Remark 1}

In the nonlinear case, Theorem $I$ is to be compared with Corollary 2.1 of section 2.4.1. Adaptation has increased the robust stability margin by allowing the arbitrary scalar $\gamma$. In the memoryless case, this can be interpreted as allowing the adaptive controller to seek a linearisation around the operating point.

In the linear higher order case, the criteria for the non-adaptive controller is in terms of fixed $A$ and $B$ (i.e. the nominal plant) while the adaptive self-tuner allows any $A$ and stable $B$ that satisfies the conditions. If $A_{0}$ and $B_{0}$ are known, it is of course possible to get necessary and sufficient conditions in the non-adaptive case. The presence of the nonlinear $\mathrm{B}_{\mathrm{cp}}$ however, only allows sufficient conditions in the adaptive case.

In fact, even if it were assumed that the true plant is linear, $B_{0}$, A will probably not be known. A less restrictive assumption would be that $\| M_{1}^{-1}-1$ is known i.e.

$$
\sup _{|q|=1}\left|\frac{A}{B} 0-\frac{A}{B}\right|=g_{m}
$$

Bence in the non-adaptive case, we require that

$$
\sup _{|q|=1}\left|\frac{E B}{P C}\right| g_{m}<1 \text { : for a fixed } A, B
$$

while in the adaptive case, we only require that there exists $A$ and stable B such that

$$
\sup _{|q|=1}\left|\frac{E B}{P C}\right| g_{m}<\left(1-\sigma_{0}\right)^{t 1 / 2}
$$


ROBUSTNF.SS OF THF. ADAPTTVF. CONTROTITIF.R

Note that $g_{m}$ ' is a function of $A$.

T.t does not appear possible to state a case for an adaptive a.s opposed to an non-adaptive controlier from robust. stabilit.y considerations alone. Tn principle, given a nominal. plant $\frac{B}{A}$ and a range of possible plant perturbations (linear) represented by $g_{m}$ " we can always desjgn $P$ and $C$ to ensure stabjlity, without using adaptation. It may however be that the resulting $P$ and $C$ does not meet other design criteria eg. the resulting reference model $\frac{1}{\mathrm{p}}$ may be too slow. In such a case, an adaptive controller may be viewed as introducing an extra degree of freedom in the design. Tf. a particular reference model, $\frac{l}{p}$ is desired for instance, then Eqn. 51 ajlows more flexibility in meeting stability requirements than does Eqn. 50.

Remaxk 2

A defect in the criteria is the dependence on an a priori unknown scalar $\sigma_{0}$. Some properties of the sequence $\{\sigma\}$ are discussed latex in this chapter. It is worth noting that $\sigma$ is easily computed on-line. A reasonable design strategy (confirmed by simulation in Chaptex 5 ) is to assume that $\sigma_{0}$ is small when choosing $P$ and $C$.

\section{Remark 3}

Theorem 1 b) provides a frequency domain test for $l_{\infty}$ stability. Note that the test is only possible when an estimator with a non-unity forgetting factor is used. For the small gain theorem to be valid, the associated system must still be stable for the scalar $\alpha$ chosen. This is easy to check because the magnitude of each root (simple or complex) of the numerator and denominator is increased by $\exp (\alpha)$ in the associated 
ROBUSTNF.SS OF THF, ADAPTIVF, CONTROIST.F.R

system. Recall that the $I_{2}$ gain of a linear ARMA model, $I_{(}\left(q^{-1}\right)$, is given by $\sup _{|q|=1}\left|I\left(q^{-1}\right)\right|$. For the associated operator, $L^{*}$, the $I_{2}$ gain is

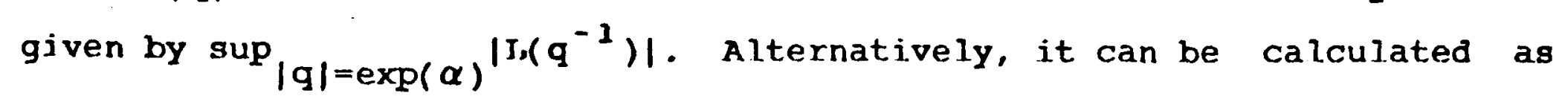
$\sup _{|q|=1}\left|I_{1}\left([\exp (-\alpha) q]^{-1}\right)\right|$.

\subsubsection{Input Frror $(y=0)$}

In Chapter 2 it has been shown that when the true plant is linear, but of higher order than the assumed plant, we can represent it either with an input or an output error model without any difference to the robust stability analysis. Hence the input error model is useful mainly when $M_{2}$ is nonlinear.

From Fig. 3-8, we have:

$$
\begin{aligned}
& \mathrm{H}_{i}=\frac{\mathrm{AE}}{\mathrm{PC}} \frac{\mathrm{PB}}{\mathrm{A}}\left(M_{2}{ }^{-1}-1\right) \frac{\mathrm{A}}{\mathrm{PB}} \\
& \operatorname{gain}\left(\mathrm{H}_{i}\right) \leqslant \sup _{|q|=1}\left|\frac{\mathrm{AE}}{\mathrm{PC}}\right|\left\|\mathrm{M}_{2}{ }^{-1}-1\right\|_{\Delta}
\end{aligned}
$$

when $M_{2}^{-1}-1$ is time-invariant, memoryless and has a continuous Frechet derivative over its domain. (from Temma 1, Section 3.4.1)

The gain of the associated system of exponentially weighted quantities is by analogy to the previous section:

$$
\operatorname{gain}\left\{\mathrm{H}_{\mathrm{i}}{ }^{\star}\right\} \leq \sup _{|q|=1}\left|\left\{\frac{F A}{P C}\right\}^{\star}\right|{\| M_{2}}^{-1}-1 \|_{\Delta}
$$

\section{Theorem 1}

The results in Theorem 1 for the output error case (section 3.4.1.) hold for the input error case when $M_{2}^{-1}-1$ is restricted as for Eqn. 53 above. 
ROBUSTMF.SS OF THF, ADAPTTVF, CONTROTITF.R

\section{Remark}

In so far as robust stability is concerned, there is no difference between the input and output error cases when the nonlinearity is restricted as above. This is similar to the situation in the non-adaptive case. Note however that this is true only when $Q=0$ and is not necessarily true when $Q \neq 0$. Note too that if the nonlinearity is such that we cannot apply Iemma 1 of section 3.4 .1 , then in Eqn. 53, the gain corresponding to the purely jinear part of $B_{i}$ will be different from the output error case: because the operators which constitute ${ }_{i}$ will not commute.

\subsubsection{Examples}

Some examples will be used to illustrate the preceding results.

\section{Fxample 1 (Nonlinear)}

Consider the nonlinear example of section 2.6.2. Assume that the linear subsystem is minimum phase. Table 1 of section 2.6 .2 lists the sector conditions for the nonlinearity (which may be at the input or output), in order that the non-adaptively controlled system is stable.

For the same range in the $P$ design polynomial, and with $c=1$, Table 1 below lists the sector bounds on the nonlinearity which meets the gain conditions of the Theorems in section 3.4. $\sigma_{0}$ is arbitrarily assumed to be 0.1 . As in the non-adaptive case, a slower $\frac{1}{\mathbf{p}}$ enlarges the sector bounds on $\mathrm{M}_{\mathbf{i}}$. 
ROBUSTNESS OF THF, ADAPTIVF: CONTROT,T.F.R

\section{Table 1}

Varjation of sector bounds with $\mathrm{P}$ for Adaptive Controller

$\begin{array}{llll}\text { p } & \frac{F A}{P C} & \begin{array}{c}\text { Radius of } \\ \text { Conic Bounds }\end{array} & \text { Sector Bounds } \\ 0 & 1.7 & .56 & (0.64 \gamma, 2.26 \gamma) \\ 0.2 & 1.42 & .67 & (0.60 \gamma, 3.03 \gamma) \\ 0.4 & 1.2 .1 & .78 & (0.56 \gamma, 4.59 \gamma) \\ 0.6 & 1.06 & .89 & (0.53 \gamma, 9.35 \gamma) \\ 0.8 & 1.5 & .63 & (0.61 \gamma, 2.72 \gamma) \\ 0.9 & 3.0 & .32 & (0.76 \gamma, 1.46 \gamma)\end{array}$

Similarly Table 2 below corresponds to Table 2 in section 2.6 where the $c$ polynomial (observer) is varied while $p=0.4$.

\section{Table 2}

Variation of sector bounds with $\mathrm{c}$ for Adaptive Controller

$\begin{array}{llll}\text { C } & \frac{\text { EA }}{\text { PC }} & \begin{array}{c}\text { Radius of } \\ \text { Conic Bounds }\end{array} & \text { Sector Bounds } \\ -0.9 & 12.1 & .0784 & (.93 \gamma, 1.09 \gamma) \\ -0.5 & 2.43 & .390 & (.72 \gamma, 1.64 \gamma) \\ -0.2 & 1.52 & .624 & (.62 \gamma, 2.66 \gamma) \\ 0.2 & 1.12 & .847 & (.54 \gamma, 6.54 \gamma) \\ 0.5 & 1.26 & .753 & (.57 \gamma, 4.05 \gamma) \\ 0.9 & 5.8 & .164 & (.86 \gamma, 1.20 \gamma)\end{array}$

Comparing with $p=0.4$ in Table 1 above, it is obvious that a proper choice of $C$ can enlarge the sector bounds considerably. As in the non-adaptive case, the sector bounds increase as the reference model, $P^{-1}$ becomes slower, until it is slower than the linear subsystem. 
ROBUSTNF.SS OF THF. ADAPTIVF, CONTROT,IF.R

In both tables above, the arbitrary scalar factor, $\gamma \neq 0$, allows an extra degree of flexibility in the sector bounds.

If an estimator with a forgetting factor $\beta=0.99$ is used, then to ensure that the system is also $1_{\infty}$ stable, we need to consider the associate system with a weighting factor of $\alpha<0.005$. It is obvious that the sector bounds in Tabjes 1 and 2 will only be very s]ightly different. In this case then, robust $I_{2}$ stability implies virtually the same robust $1_{\infty}$ stability.

\section{Example 2 (Unmodelled fractional delay)}

Consider the tank fluid temperature control problem discussed in Appendix A.3 of Pranklin and Powell[35]. With a non-zero time constant of $\frac{1}{2}$ and a steady state gain of 10 , the continuous time plant is

$$
\frac{20 e^{-\tau s}}{s+2}
$$

Assume that the delay $\tau$ is in the range $0.1 T \leqslant \tau \leqslant 0.5 T$ where $T$ is the sampling period. If the fractional delay is ignored, then the equivalent discrete-time system, with an input zOH, and for a sampling period $T$ of 0.1 is:

$$
\frac{q^{-1} b}{1-0.819 q^{-1}}
$$

where $b=1.81$ if accurately derived.

If the non-adaptive clarke-Gawthrop self-tuner discussed in Chapter 2 is used for this system, with the nominal plant of Fqn. 56, Corollary 2.2 of section 2.4 .1 says that the closed loop system is stable if the Nyquist locus of ${ }_{0}\left(q^{-1}\right)$ does not encircle $-1+$ jo. Fig. 3-9 
ROBUSTNF.SS OF THF: ADAPTIVE CONTROI,TIF.R

shows that even when a slow reference model is used $\left(P=10-9 q^{-1}\right)$, the closed loop system is only stable for a restricted range of $b$ when $\tau=0.4 \mathrm{~T}$.

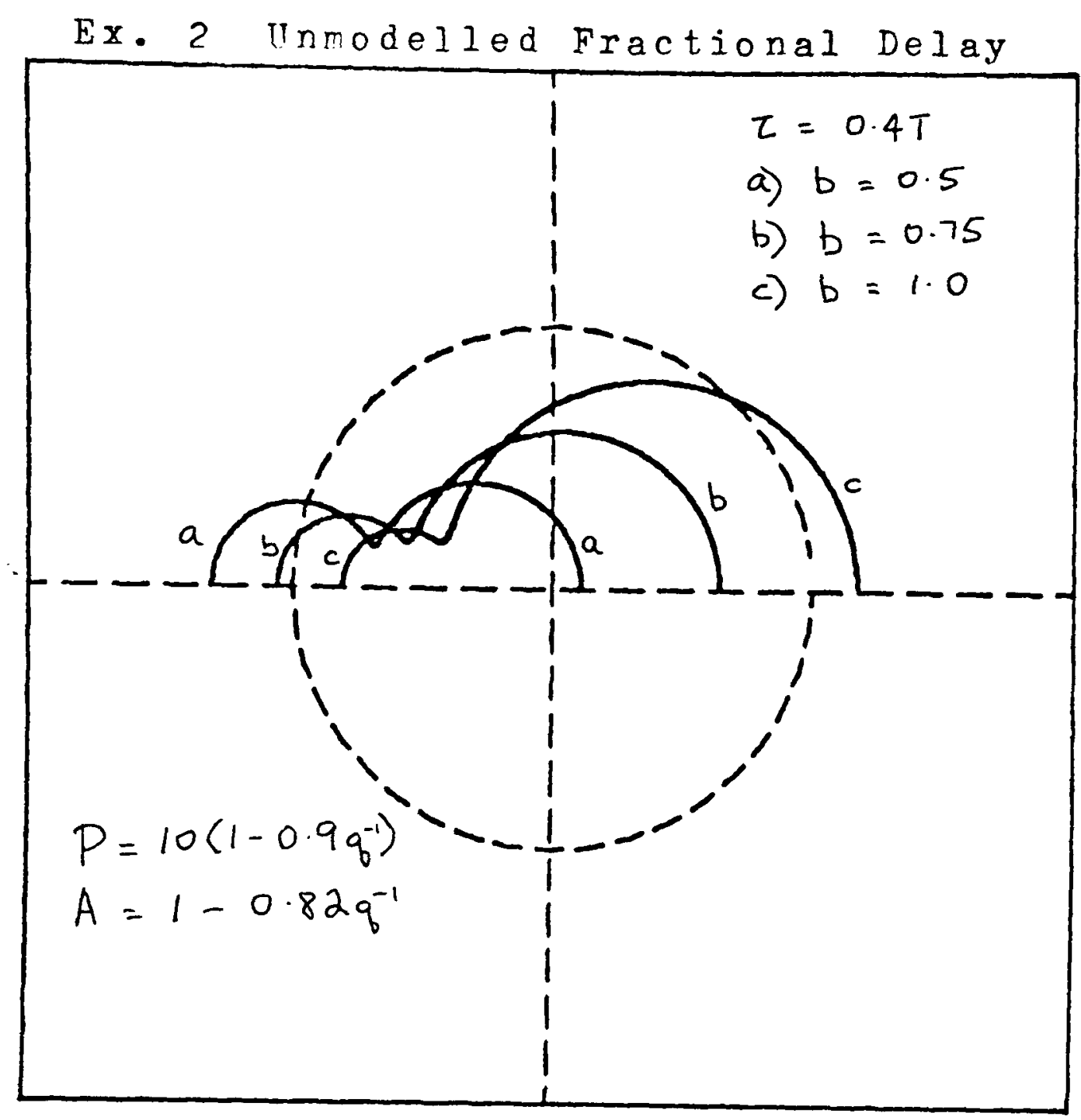

Fig. 3-9 Vyquist Loci for Varying Jominal Gain

When the adaptive implicit self-tuner is used, the system is unconditionally stable for all delays in the range, when $P=10-9 q^{-1}$. Fig. 3-10 shows Nyquist plots for $T=0.3 T, 0.4 T$ and $0.5 T$. Each plot corresponds to a different $B$ as the theorems in section 3.4 only require the existence of one. 
ROBUSTNF.SS OF THF, ADAPTIVF, CONTROTIT.F.R

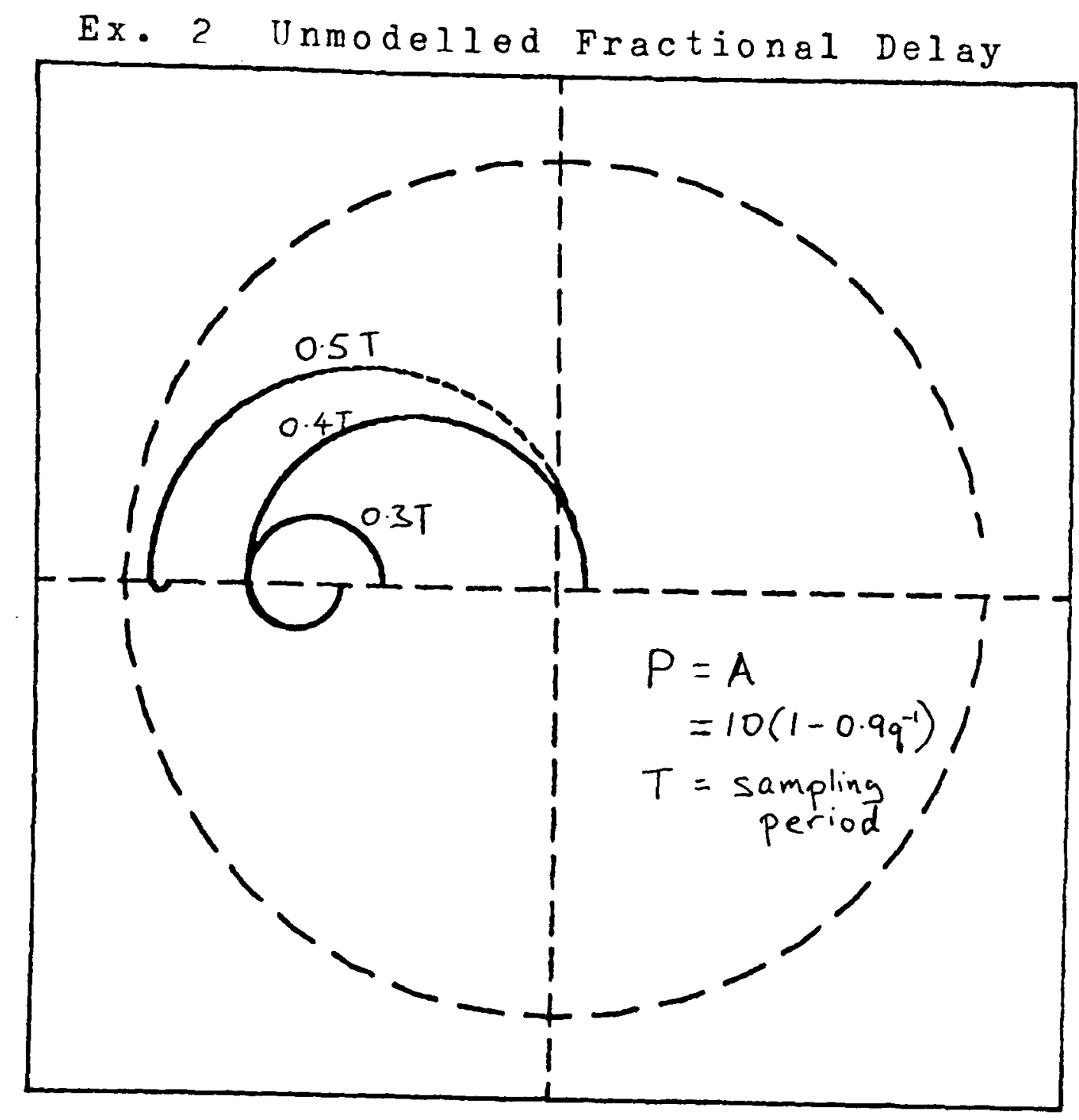

Fig. 3-10 Nyquist Loci for varying Fractional Delay

\section{Example 3 (Reduced order mode1)}

In Goodwin and Ramadge[45] an example is given of a minimum variance reduced order self-tuner applied to a second order linear system. The resulting closed loop system was described by

$$
\begin{aligned}
& A_{0}=1-1.6 q^{-1}+0.8 q^{-1} \\
& B_{0}=1.6+0.2 q^{-1} \\
& k=1
\end{aligned}
$$

The controller is designed as if the system is first order, i.e.

$$
\begin{aligned}
& B=b \\
& A=1-a q^{-1}
\end{aligned}
$$


ROBUSTNE.SS OF THE ADAPTIVF. CONTROI,T.F.R

For a minimum variance controllex, $P=1$. In view of Goodwin's results, it is not surprising that it is imposgible to find an $A$, $B$ such that the Nyquist locus of $A_{0}$ in section 3.4 .1 is within the critical circle, Fig. 3-11a.

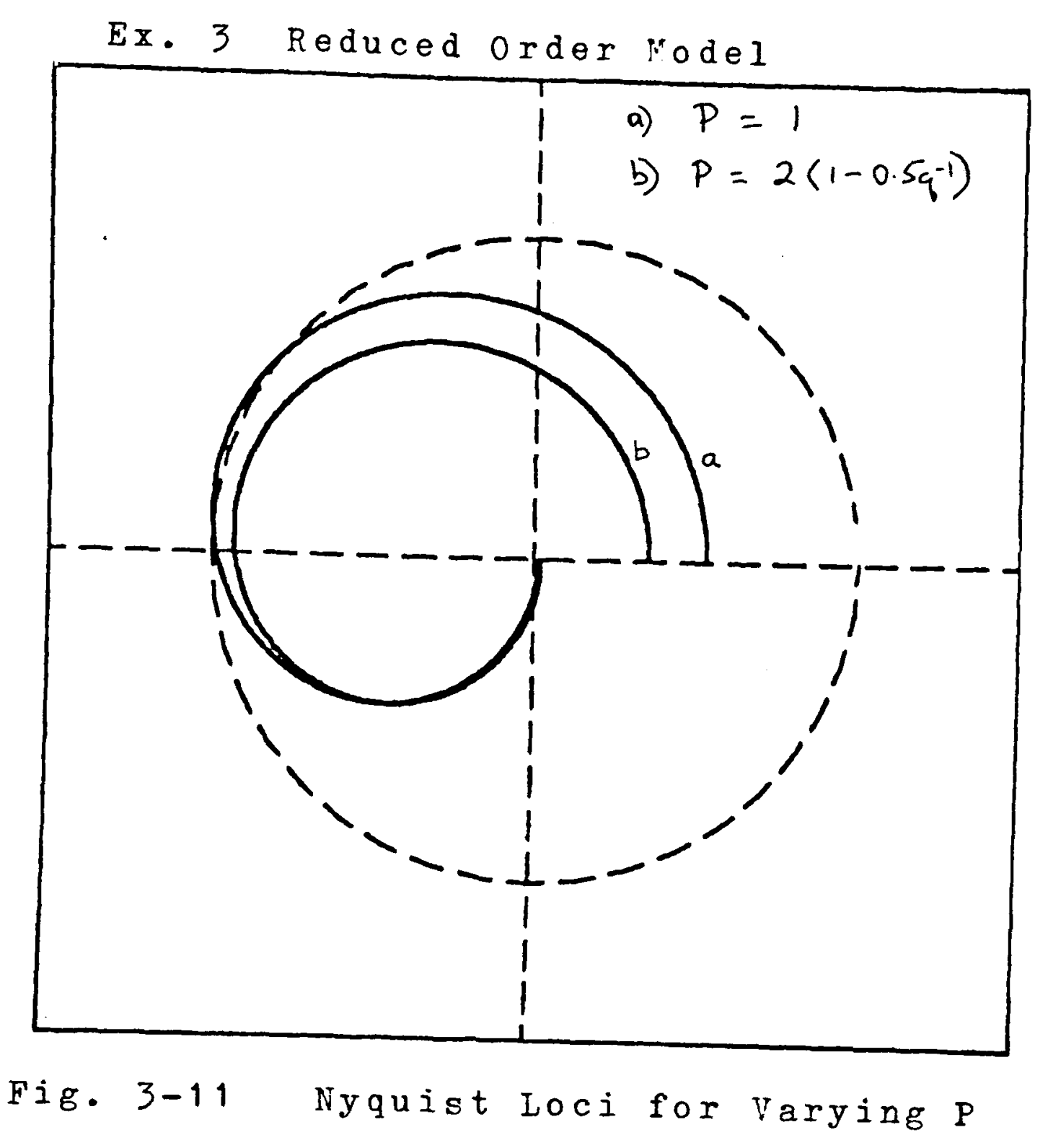

For a slower P, this is possible. Fig. 3-1lb shows a Nyquist plot of $\mathrm{F}_{0}$ when $\mathrm{P}=2-\mathrm{q}^{-1}$, corresponding to $\mathrm{B}=1.0$ and $A=1-0.9 q^{-1}$.

Note that when $Q=0$, the stabilising effect of $P$ is identical to $c$. Since $C$ corresponds to the observer poles, there would of course be a difference in disturbance attenuation properties. 
ROBUSTNF.SS OF THF. ADAPTIVF, CONTROTITFR

\subsection{INSTABTI,ITY OF THF. ADAPTIVF. AT,GORITHM}

All the preceding results for the stability and robustness of the adaptive self-tuner are only in terms in sufficient conditions. It is of interest to see if sufficient conditions can also be obtained for the instability of the adaptive controllers.

It is shown in this section that for linear plant perturbations, it is possible to show both $I_{2}$ and $I_{\infty}$ instability. In the self-tuning context, it is obviously the latter result that is of practical interest. These results not only confirm a well known condition for the non-robustness of nonminimum phase systems under implicit self-tuning control, but also specify the disturbances or setpoints which will lead to "blow-up".

In section 3.2.1, it has been shown that in the linear full order case with no $c$ estimation, the estimated prediction error $\hat{e}_{1}(t)$ is always bounded (assuming $\sigma_{0}<1$ ). Instability in this case arises when $[P B+Q A]^{-1}$ has unstable poles eg. when $Q=0$ and the plant is non-minimum phase (See Eqn. 22). In that case, it is always possible to find a disturbance sequence $d(t)$ or setpoint $w(t)$, which will 'activate' the unstable poles of the control law.

If the plant is linear, but of different order to that assumed by the self-tuner, then it can be shown that the estimated prediction error, $\hat{e}_{1}(t)$ will not be bounded $\left(I_{2} / I_{\infty}\right)$ for certain disturbances or setpoints if $\left[\mathrm{PB}{ }_{0}+\mathrm{QA}_{0}\right]^{-1}$ has unstable poles. For convenience, Fig. 3-12. shows the error feedback system for the cage of the implicit self-tuner. It is derived from Fig. 3-5, for linear perturbations. 

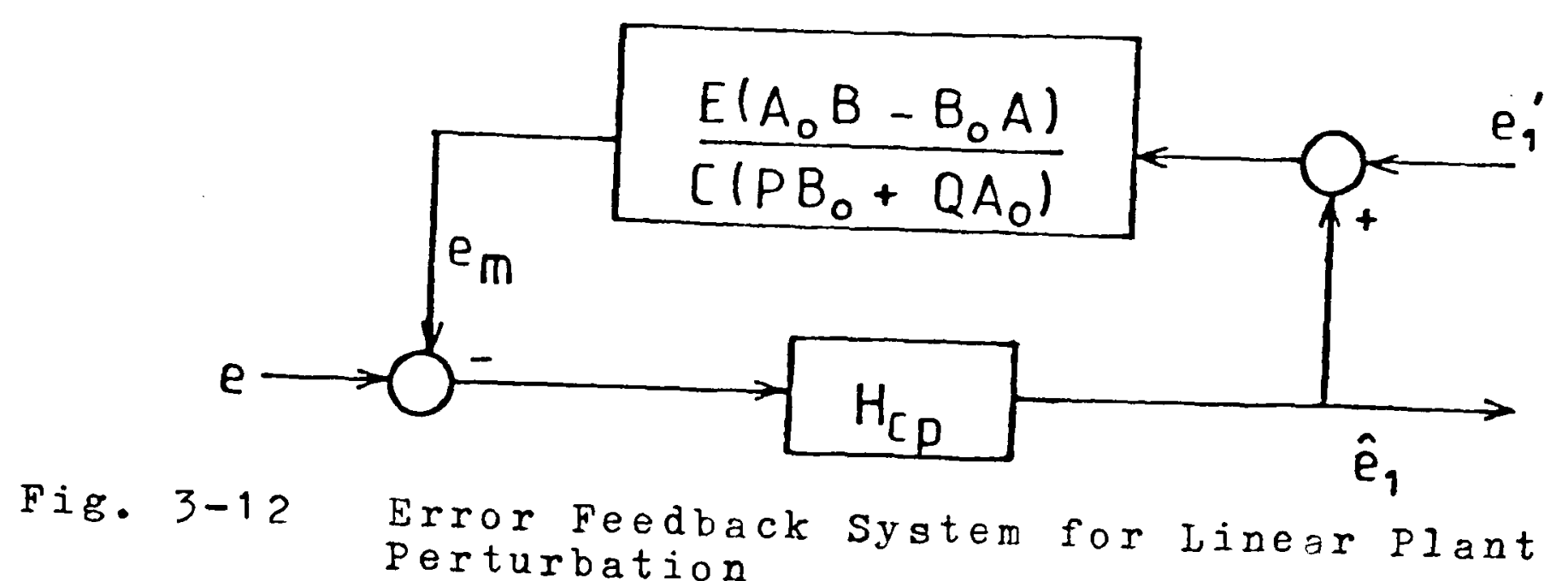

Theorem 1 below formalises an intuitively obvious result. The orthogonal decomposition approach developed by Takeda and Bergen[80] is used. All the inputs, outputs and error signals are assumed to be elements of $I_{2}$ or $I_{2} e^{*}$ If $R_{0}$ (Fig. 3-12) has unstable poles, then the space of signals, $1_{2} e^{\prime}$ can be decomposed into complementary subspaces, $1_{2}$ ' and $1_{2}^{1}$, which are orthogonal and defined such that for every $x \in 1_{2}^{\prime}$, ${ }_{0}\{x\} \in 1_{2}$ and for every $x \in 1_{2}^{1}, H_{0}\{x\} \notin 1_{2}$. In this case, a simple example of $x_{1} \in 1_{2}^{\prime}$ is when $x_{1}(t)=\left[P B_{0}+Q A{ }_{0}\right] v(t)$, where $v(t)$ is an arbitrary sequence in $1_{2}$. If $x_{2}(t)$ is the sequence such that $\sum^{\infty} x_{1}(t) x_{2}(t)=0$, then $x_{2} \in 1_{2}^{1}$.

\section{Theorem I.}

Assume that $\left[\mathrm{PB}{ }_{0}+Q{ }_{0}\right]^{-1}$ has unstable poles.

a) If there exists $A, B$ such that

$$
\sup _{|q|=1}\left|\frac{E}{C} \frac{\left(B A_{0}-A B_{\alpha}\right)}{\left(\overline{P B}_{0}+Q A_{0}\right)}\right|<\left(1-\sigma_{0}\right)^{+1 / 2}
$$

then $\hat{e}_{1}(t) \in I_{2}$ for some input sequence $e_{1} \cdot(t) \in I_{2}^{1}$ and $e(t)$ such that

$$
\| e_{1}(t)>\delta ! e(t) \text { where } \delta \text { is a constant. }
$$

b) $1_{\infty}$ instability 
If there exists a constant $\alpha<0$ such that

i) $\left(\left[P{ }_{0}+Q A_{0}\right]^{-1}\right)^{\star}$ has unstable poles, where ${ }^{\star}$ denotes the associated polynomial as defined in F.qn. 43

i i) $\sup _{|q|=1} \mid \frac{E^{*}\left(B^{*} A_{0}^{*}-A^{*} B_{0}^{*}\right)}{C^{*}\left(P^{*} B_{0}^{*}+Q^{*} A_{0}^{*}\right)}<\left(1-\sigma_{0}\right)^{+1 / 2}$

then $\hat{e}_{1}(t)$ is not $1_{\infty}$ bounded for some input sequence $e_{1}(t)$, $e(t) \epsilon 1_{\infty} ; e_{1}{ }^{\star} \epsilon I_{2}^{1}\left(\theta_{0}^{*}\right)$ and $e_{1} .^{\star}\left\|, \delta H e^{\star}(t)\right\|$.

\section{Proof}

Part (a) merely uses the small gain theorem for instabjility first proved by Takeda and Bergen[80]. It is proved again in the Appendix B.4.

Part (b) is an extension of the exponential weighting instability results of Vidyasagar[83] to the discrete-time case. In Appendix B.4.

$\mathbf{\square}$

Remarks

Theorem 1 complements the robust stability results of section 3.4 by showing that the same frequency domain conditions produce stability or instability according to whether $\left[\mathrm{PB}_{0}\right]^{-1}$ is stable or unstable (when $Q=0)$. Note that determining the gain of $H_{0}$ can still be done via the Nyquist locus whether $H_{0}$ is stable or unstable, with the difference that if it is unstable, then the $\sup _{|q|=1}$ is to be interpreted as a conditional gain, valid only for inputs to $\mathrm{H}_{0}$ which belong to ${ }_{2}{ }^{\prime}$.

In the main, Theorem 1 sets out formally a fact which is often mentioned in the literature, but not explicitly shown i.e. the instability of non-minimum phase systems controlled by an implicit sejf-tuner. Theorem 1 also shows specifically the inputs which will 
ROBUSTNF.SS OF THF, ADAPTIVF, CONTROT,TER

result in instability.

The test for $1_{\infty}$ instability is as simple as that for $1_{\infty}$ stability discussed in section 3.4.1: $\sup _{|q|=1}\left|\mathrm{H}_{0}{ }^{*}\right|$ equals $\sup _{|q|=\exp (\alpha)}\left|\mathrm{R}_{0}\right|$. Note that for instability, the limitation on possible $\alpha$ is only that the associate operator, $H_{0}^{*}$ must still have unstable poles for the $\alpha$ chosen, whereas in the stability case, $\alpha$ must ensure that $B_{0}^{*}$ is still stable and the estimator, $H_{c p}{ }^{*}$ is still dissipative $[1>\beta \exp (2 \alpha)]$.

As for the robust stability case, the conditions of Theorem I are only suffjcjent. It is restrictive in that it requires $\left[P P_{0} Q_{0}\right]^{-1}$ to have unstable poles. Since $A_{0}, B_{0}$ are not known, Theorem $I$ cannnot be used as a design aid. Furthermore, by analogy to the non-adaptive case of Chapter 2, instability should depend on the poles of $\left[1+\mathrm{B}_{0}\right]^{-1}$ rather than those of $H_{0}$. The presence of the estimator ( ${ }_{c p}$ ) prevents such a simple extension of the non-adaptive results, except where the parameter estimates are asymptotically time-invariant. We conclude this section with IJemma 1 below.

\section{I.enma I}

Assume that there exists some $\theta^{*}$ such that $\lim _{t \rightarrow \infty} \hat{\theta}(t)=\theta^{*}$. If the non-adaptive self-tuner of Corollary 2.3, section 2.4.3 corresponding to $\theta^{*}$ is unstable, then the adaptive implicit self-tuner is asymptotically unstable.

Proof In Appendix B.4. 


\subsection{F.XPLICIT AT,GORITHMS: STABIJITTY AND ROBUSTNFSS}

The adaptive algorithm analysed for robustness in the previous sections of this chapter is often known as an implicit or 'direct. algorithm. This is because the control problem is formulated so that the estimator estimates the parameters of the control law directly.

Another class of algorithms, the explicit algorithms (cf Chapters 1, 2), are formulated so that the parameters of a linear ARMA model representing the system are directly estimated. The control law is then calculated using the estimated coefficients as if they were the true coefficients.

In this section, the reason why even stability results for explicit algorithms are not common is explained. Published results have only been obtained at the cost of relatively severe restrictions. A special case is studied (by assuming an all-pole, no-zero nominal plant) for which it is possibje to get not only stability but robustness results. These indicate that for nonminimum phase plants, even the explicit pole placement type self-tuners are not robust unless the order of the plant is known accurately.

The same estimation algorithm (Eqns. I to 3 ) can be used to estimate the coefficients of the model. Tret the data vectors $x(t), x_{0}(t)$ be the same as those defined for the implicit self-tuning controller. Iet the parameter vector be

$$
\theta_{p}^{T}=\left[-a_{1},-a_{2}, \ldots \cdot b_{0}, b_{1}, \ldots . .\right]
$$

Then if the assumed plant is

$$
\begin{aligned}
& \qquad y(t)+a_{1} y(t-1)+\ldots+a_{n} y(t-n)=b_{0} u(t-1)+\ldots+d(t) \\
& \text { we can write } y(t)=x^{T}(t-1) \theta_{p}+d(t)
\end{aligned}
$$


ROBUSTNF.SS OF THF. ADAPTIVF. CONTROITIF.R

and

$$
y_{0}(t)=M_{1} y(t) \quad \text { : for output error case }
$$

To represent extra delays, the leading coefficients of $B\left(q^{-1}\right)$ may of course be zero.

The prediction error, $\hat{e}_{1}(t)$, that goes into the parameter update, E.qn. 1 , is :

$$
\hat{e}_{1}(t)=y_{0}(t)-\hat{y}(t / t-1)
$$

where the one step ahead prediction for $y$ is

$$
\hat{y}(t / t-1)=x_{0}^{T}(t-1) \hat{\theta}_{p}(t-1)
$$

The control law may then be computed explicitly, according to some design strategy for known plants such as the pole placement methods of Sections 2.4.4 and 2.4.5, using the estimated parameter vector, $\hat{\theta}_{\mathrm{p}}$ instead of $\theta_{p}$.

Define the error quantities in an analogous manner to the implicit case.

$$
\begin{aligned}
e_{p}(t) & \cong x_{0}{ }^{T}(t-1)\left[\hat{\theta}_{p}(t)-\theta_{p}\right] \\
& =x_{0}{ }^{T}(t-1) \bar{\theta}_{p}(t-1)
\end{aligned}
$$

Since the same estimator is used as in the implicit case, Lemmas 1-3 of section 3.1 are valid here too.

\section{Ininear Full order case under Explicit Control}

Consider the stability of the linear, full order case, corresponding to section 3.2 .1

$$
\begin{aligned}
& M_{1}=1 \text { i.e. } y_{0}=y \text { and } x=x_{0} \\
& \hat{e}_{1}+e_{p}=y(t)-x^{T}(t-1) \theta_{p}=d(t): \text { from Eqn. } 64
\end{aligned}
$$

Lemmas 2 and 3 of section 3.1 show that if $d(t)$ is bounded in the $l_{\infty}$ or 
ROBUSTNF.SS OF THF. ADAPTIVF. CONTROT,T.F.R

$1_{2}$ sense, then $\hat{e}_{1}(t)$ is bounded in the $1_{\infty}$ or $1_{2}$ sense.

Unlike the implicit case considered in section 3.2.1, stability of the prediction error does not automatically imply that the output $y$ is stable. From Eqns. 66 to 67 we can write

$$
\begin{aligned}
& \hat{e}_{1}(t)=y(t)-[1-\hat{A}(t-1)] y(t)-\hat{B}(t-1) u(t-1) \\
& =\hat{A}(t-1) Y(t)-\hat{B}(t-1) u(t-1) \\
& \text { i.e. } \hat{A}(t-1) y(t)=\hat{B}(t-1) u(t-1)+\hat{e}_{1}(t) \\
& \text { where } \hat{A}(t-1)=1+\hat{a}_{1}(t-1) q^{-1}+\hat{a}_{2}(t-1) q^{-2}+\ldots
\end{aligned}
$$

F.qn. 71 describes the linear time-varying system of the identified model. Hence the control law must stabilise the identified model for overall stability of the system.

In the pole placement method of Astrom and Wittenmark[10],[11], if no attempt is made to cancel any open loop zeroes, the control law is given by (cf Chapter 2)

$$
\hat{R}_{1}(t) u(t)=T_{A} w(t)-\hat{S}_{A} Y(t)
$$

where $\hat{R}_{1}$ and $\hat{s}_{\hat{A}}$ are the estimates of the polynomials defined in Eqns. 2-60, 2-61 and obtained from the solution of

$$
\mathrm{CA}_{\mathrm{m}}=\hat{\mathrm{A}}_{1}+\mathrm{q}^{-1} \widehat{\mathrm{BS}}_{\mathrm{A}}
$$

With the control law above, acting on the time-varying system of Eqn. 71, it appears impossible, in general, to prove stability without extra assumptions, discussed in section 3.6.3. However some special cases are discussed in the following section. 
ROBUSTNF.SS OF THF. ADAPTIVE CONTROTIIF.R

\subsubsection{Special Cases}

A trivial case is when the feedback part of the control law F.gn. 73 i.s of zeroth order i.e. $\operatorname{deg}\left\{\hat{R}_{l}\right\}=\operatorname{deg}\left[\hat{S}_{A}\right\}=0$. The degrees of $C_{1} A_{m}$, $\hat{A}$ and $\hat{B}$ must of course be selected to ensure that Fgn. 74 has a solution. The control law may then be written as

$$
\hat{r}(t-1) u(t-1)=T_{A} w(t-1)-\hat{g}(t-1) Y(t-1)
$$

Substituting F.qn. 75 in F.qn. 73 and using Eqn. 74

$$
\begin{aligned}
\hat{r}(t-1) \hat{e}_{1}(t) & =\hat{r}(t-1) \hat{A}(t-1) y(t)-\hat{B}(t-1)\left[T_{A} w(t-1)-\hat{s}_{(t-1) y(t-1)]}\right. \\
\text { or } y(t) & =\left[C A_{m}\right]^{-1}\left[\hat{x}(t-1) \hat{e}_{1}(t)+\hat{B}(t-1)\left[T_{A} w(t-1)\right]\right]
\end{aligned}
$$

Since $C$ and $A_{m}$ are selected to be stable, and the scalar $\hat{r}$ is uniformiy bounded, (from Eqn. 74, if $\hat{A}$ is monic), stability of $\hat{e}_{1}(t)$ implies stability of $y(t)$ in this case.

A less restrjctjve case is when only $\operatorname{deg}\left\{\hat{R}_{1}\right\}=\operatorname{deg}\{\hat{B}\}=0$. i.e. the control law, Fqn. 73 is a 'moving average' of past outputs and setpoints and the estimated model in Eqn. 71 is 'autoregressive' in $y$ i.e.

$$
\begin{aligned}
& \hat{\hat{A}}(t-1) y(t)=\hat{b}(t-1) u(t-1)+\hat{e}_{1}(t) \\
& \text { and } \hat{x}(t-1) u(t-1)=T_{A} w(t-1)-\hat{S}_{A}(t-1) y(t-1)
\end{aligned}
$$

From Eqns. 77,78 and Eqn. 74

$$
y(t)=\left[C A_{m}\right]^{-1}\left[\hat{r}(t-1) \hat{e}_{1}(t)+\hat{b}(t-1)\left\{T_{A} w(t-1)\right]\right]
$$

In this case, stability of $\hat{e}_{1}(t)$ is sufficient for stability of the closed loop system.

This result is perhaps less surprising if we observe that for $B=1$, and $\operatorname{deg}\left\{\hat{R}_{1}\right\}=0$, the explicit pole-placement algorithm derives the control law parameters from the solution of (cf Eqn. 74)

$$
C A_{m}=\hat{A}_{1}+q^{-1} \hat{S}_{A}
$$


while in the implicit self-tuner, the relationship between the control law parameters is

$$
C P=\widehat{A G}+q^{-1 \hat{F}}: \hat{G}=\hat{E}
$$

If $P=A_{w^{\prime}}$ the feedback portions of the control laws are identical. ( $\hat{G}$, $\hat{F}$ in implicit case $=\hat{R}_{1}, \hat{S}_{A}$ respectively in explicit case) .

This last case may be less restrictive than it appears to be at first sight. It has been assumed that the plant is accurately represented by the Iinear ARMA model of Eqn. 63. It is well known that if the plant zeros are minimum phase, this model can also be written as an autoregressive model in output $y$, at the cost of a larger number of coefficients. If it is assumed that $y(t)=0$ for $t<0$, then at $t$ ime $N$, the AR model equivalent to the ARMA model of Eqn. 63 is of dimension $N$. It would of course be absurd to attempt to estimate every parameter of this AR model. If an AR model is assumed (of fixed, finite order), then the controller is in effect a reduced order controller. In such a case an equation like Eqn. 79, with fixed poles, allows a robustness analysis of this reduced order controller.

Robustness of Exp].icit Algorithm (Special Case)

Consider the output error case only (the input error case being very simjlar).

$$
\begin{aligned}
& y_{0}(t)=M_{1} Y(t) \\
& \text { Assume that } M_{1}^{-1} \text { exists. }
\end{aligned}
$$

The nominal plant is modelled as

$$
\begin{aligned}
& A\left(q^{-1}\right) y(t)=b u(t-1)+d(t) \text { :i.e. B is oth order } \\
& \text { Bence } \hat{e}_{1}(t)+e_{p}(t)=y_{0}(t)-x_{0}^{T}(t-1) \theta_{p} \\
& =d(t)-A\left(M_{1}^{-1}-1\right) y_{0}(t)
\end{aligned}
$$


ROBUSTINF.SS OF THF, ADAPTTVE, CONTROT,T.FR

With the control law specified as in Fqn. 78 , the equation corresponding to Fqn. $79 \mathrm{i.s}$

$$
Y_{0}(t)=\left[C A_{m}\right]^{-1}\left[\hat{r}(t-1) \hat{e}_{1}(t)+\hat{b}(t-1)\left\{T_{A} w(t-1)\right]\right]
$$

As $C, A_{n^{\prime}} \hat{A}$ are monic polynomials, $\hat{x}(t-1)=1$. From Fqn, $81, \quad b=\hat{b}=1$ without loss of generality $j . e$. the $b$ coefficient need not be estimated.

Irema 2, Section 3.1 together with Fqns. 82 and 83 above form the closed loop feedback system of Fig. 3-13 below.

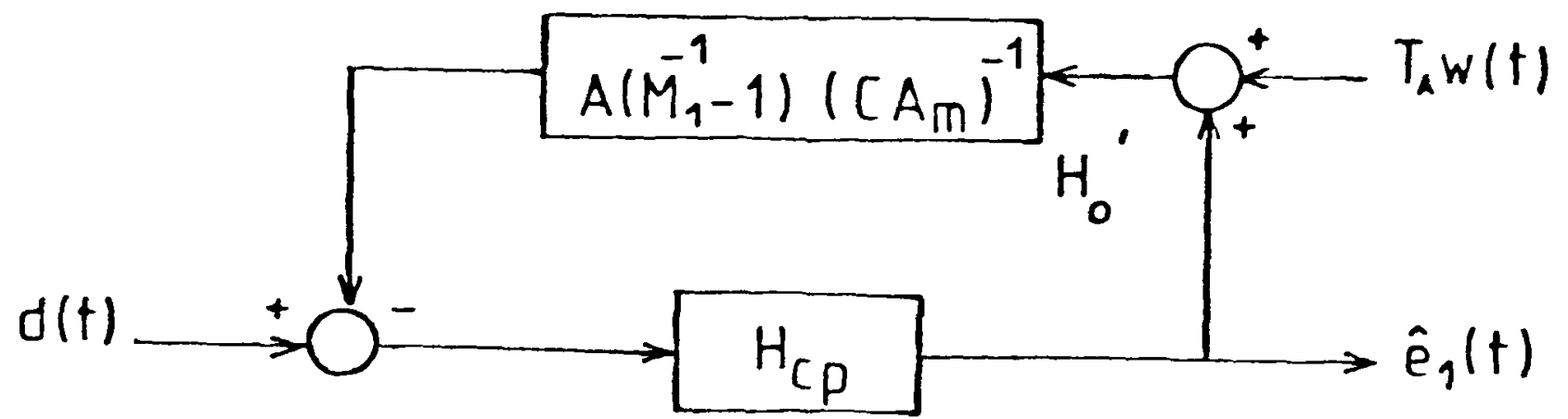

Fig. 3-13 Error Feedback System for Explicit Algorithm ( $B=$ scalar)

Note that if the system is really linear and described by Eqn. 2-1, then

$$
M_{1}=\frac{A B}{A} 0
$$

Fig. 3-13 is similar to the error feedback model in the implicit case i.e. Fig. 3-7

$$
\begin{aligned}
& \mathrm{B}_{0}=\left[\mathrm{CA}_{\mathrm{m}} \mathrm{B}_{0}\right]^{-1}\left[\mathrm{~A}_{0}-\mathrm{AB}_{0}\right]: \text { linear, higher order case } \\
& \mathrm{H}_{0} \cdot=\frac{\mathrm{A}_{\mathrm{m}}}{C A_{\mathrm{m}}}\left[\mathrm{CA} \mathrm{m}_{\mathrm{m}}\left(\mathrm{H}_{1}^{-1}-1\right) \frac{1}{C A_{\mathrm{m}}}\right] \text { : nonlinear case }
\end{aligned}
$$

Theorem I (analogous to Theorem 1 of section 3.4.1)

a) The system of Fig. 2-2, with $k=1$ and $a=0$, controlled by the explicit adaptive pole placement self-tuner described in this section is 1 stable if the following conditions hold: 


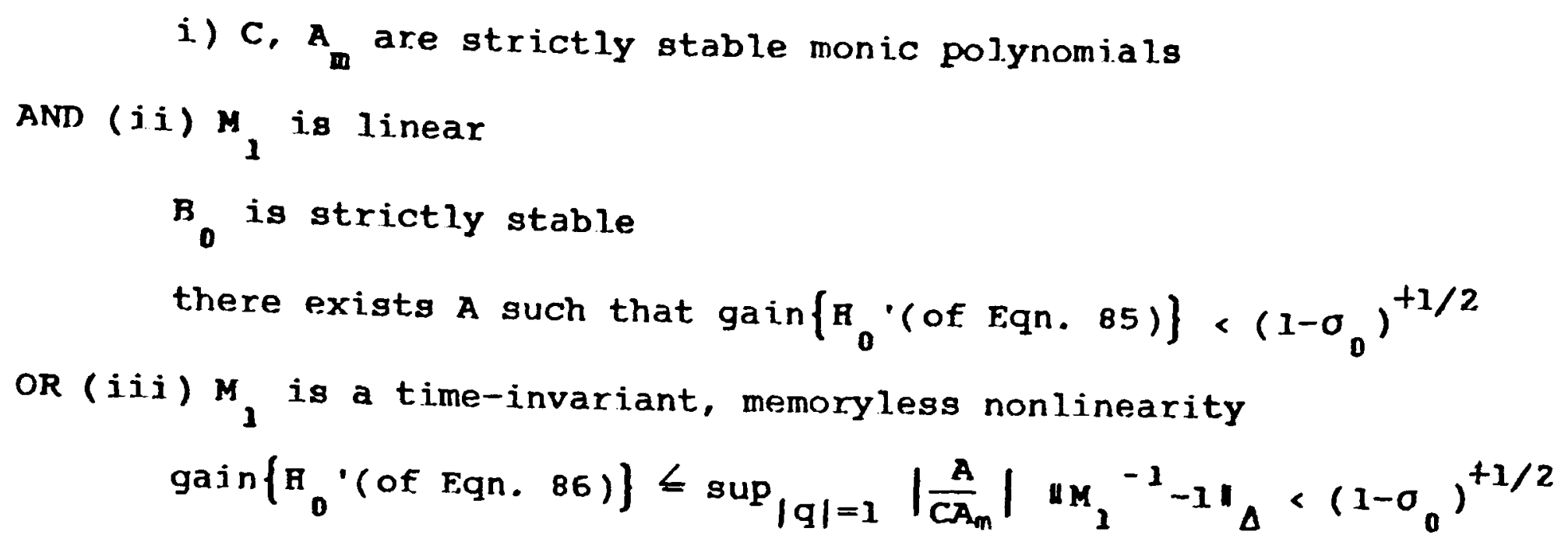

b) The same system is $l_{\infty}$ stable if the conditions in a) are met for the assocjated system i.e. for $A^{*}, C^{\star}, A_{m}^{*}$ and ${ }_{C P}^{*}$ for some $\alpha>0$ such that $1-\beta \exp (z \alpha)>0$.

Proof Similar to Theorem 1, Section 3.4.1

\section{Remarks}

When $M_{1}$ is nonlinear, the above theorem differs from the implicit self-tuner case in that no arbitrary scaling factor, $\gamma$, is allowed on the gain of $M_{1}$. This is because in this explicit algorithm, $\hat{b}$ is not estimated. If $\hat{b}$ is estimated, then the arbitrary scaling factor is permitted and the input $T_{A} w(t)$ to the feedback system of Fig. 3-13 is modified to $\hat{b}(t-1) T_{A} w(t)$.

It will be admitted that the algorithm suggested here can result in a large estimator. However, it has the advantage that there is an a priori idea of robust stability margins. There is also the possibility that the estimator can estimate an ARMA model and the estimates are then converted to those of a reduced $A R$ model, which are used in the solution of Eqn. 74 and the control law, Eqn. 78. Such a step however, would result in an estimator with properties different to those of section 3.1. 
ROBUSTNESS OF THE ADAPTIVE CONTROIIIF.R

In the special cases discussed, note that there are very few restrictions on the disturbances allowed and no assumptions are made about the asymptotic behaviour of the control law and the estimator. Unlike some stability proofs discussed in the next section, it is also not required that the estimated model, $\hat{A}, \hat{\mathrm{B}}$ be uniformly stabilisable at every instant.

As in the implicit case, robust stability depends on an inverse factor $M_{i}^{-1}-1$. This suggests that if the plant is non-minimum phase, then stability cannot be proved, even if the plant is linear, whenever the number of zeros is not exactly known. In clarke[18] it is shown that for numerical reasons, it is often desirab]e to underestimate the order of the $B\left(q^{-1}\right)$ polynomial. It would appear then that while explicit pole placement adaptive controllers can stabilise linear non-minimum phase systems, such a stability is not robust.

For the special case discussed above (Theorem 1), there is of course the instability counterpart of section 3.5 .

\section{Corollary 1 (Instability)}

In Theorem 1 above, if the plant is linear and if $B_{0}$ has one or more strictly unstable roots, then the conditions of Theorem I imply that there exists some inputs to the system,

$T_{A} w(t) \in I_{2}^{\perp}\left\{\frac{b}{C A}\left[\frac{A}{B} 0-\frac{A}{B}\right]\right)$ and $d(t)$ is chosen so that $d(t) \geqslant \delta w T_{A} w(t) *$ ( 0 a constant) such that $\hat{e}_{1}(t) E I_{2} \cdot \hat{e}_{1}(t)$ is not $I_{\infty}$ stable if. the conditions of Theorem $](b)$ are met.

Proof Analogous to that of Theorem .1 in section 3.5 
ROBUSTNF.SS OF THF. ADAPTTVF. CONTROTI.F.R

\subsubsection{F.xample}

Consider the linear system described by

$$
\begin{aligned}
& B_{0}=1-0.7 q^{-1} \\
& A_{0}=1-1.7 q^{-1}+0.72 q^{-2} \\
& k=1
\end{aligned}
$$

In Clarke[18], it is shown that if an explicit pole-placement algorithm is used to control this system, then the proximity of the zero to a pole would lead to numerical ill-conditioning. If to get around this only one $b$ parameter is estimated, then we can apply Theorem 1 to check stability.

Let the desired closed loop polynomial, $A_{m}=1$ and observer, $c=I$. From Theorem 1 , we require the Nyquist locus of $\left(\frac{\mathrm{bA}}{\mathbf{B}_{0}}-A\right)$ to be within the critical circle. Fig. 3-14 shows the Nyquist locus for a first order $A=1-0.1 q^{-1}, b=1.0$ and $a$ second order $A=\left(1-0.1 q^{-1}\right)^{2}, b=1.0$.

If however, the plant is non-minjmum phase eg. instead of Eqn. 87 we have

$$
B_{0}=1-1.2 q^{-1}
$$

then from corollary 1 above, the system is not stable if $A, b$ exists such that the Nyquist locus is within the critical circle. Fig. 3-15 shows the plots for both a first order $A$ and a second order $A$. Hence, there exists stable disturbances and/or setpoints for which the closed loop system is unstable. This is shown via a simulation in Chapter 5 . 
Section 3.6.2 Pole Placement Example

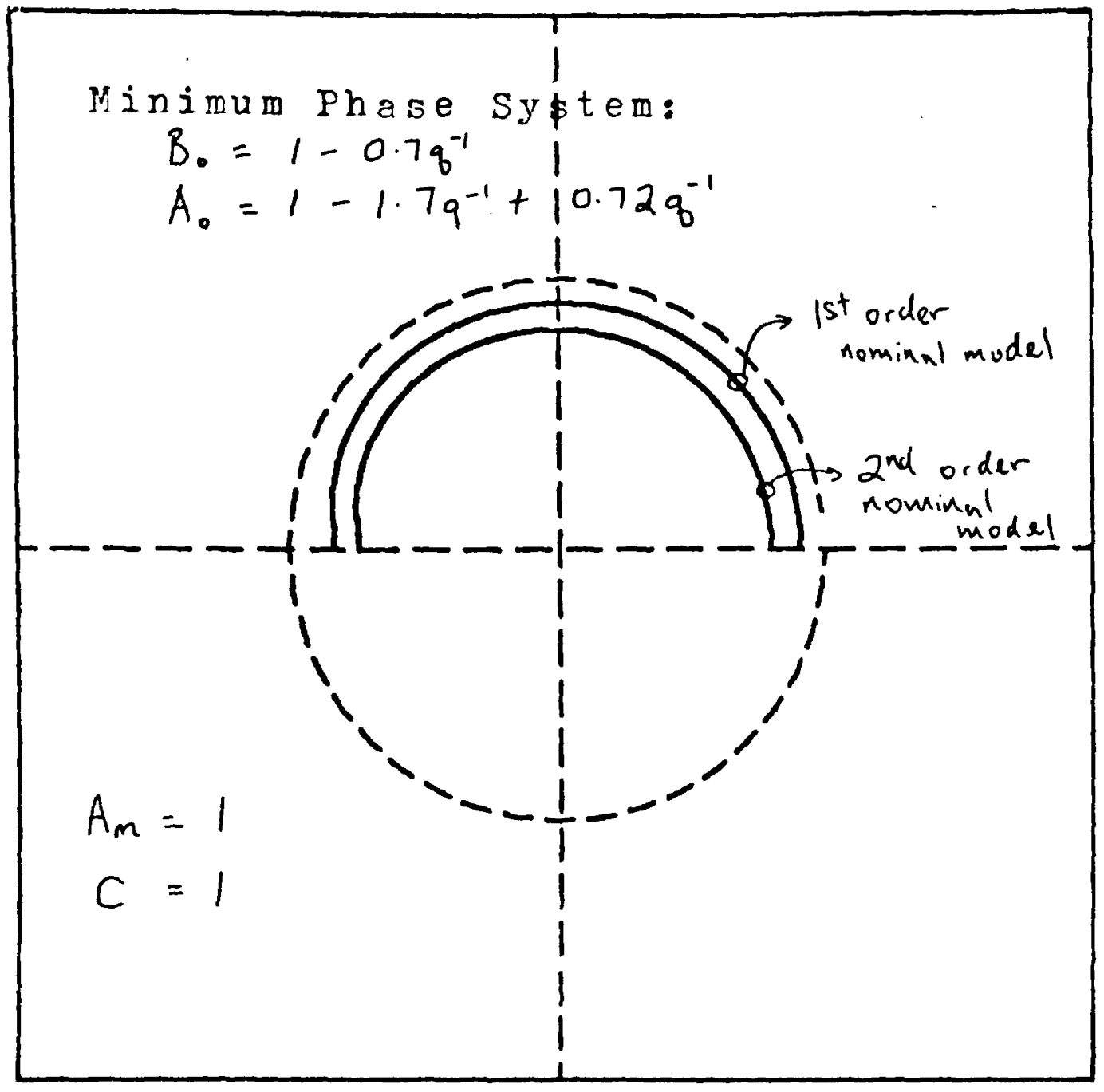

Fig. 3-14 Nyquist Loci for Varying Nominal order Section 3.6.2 Pole Placement Example

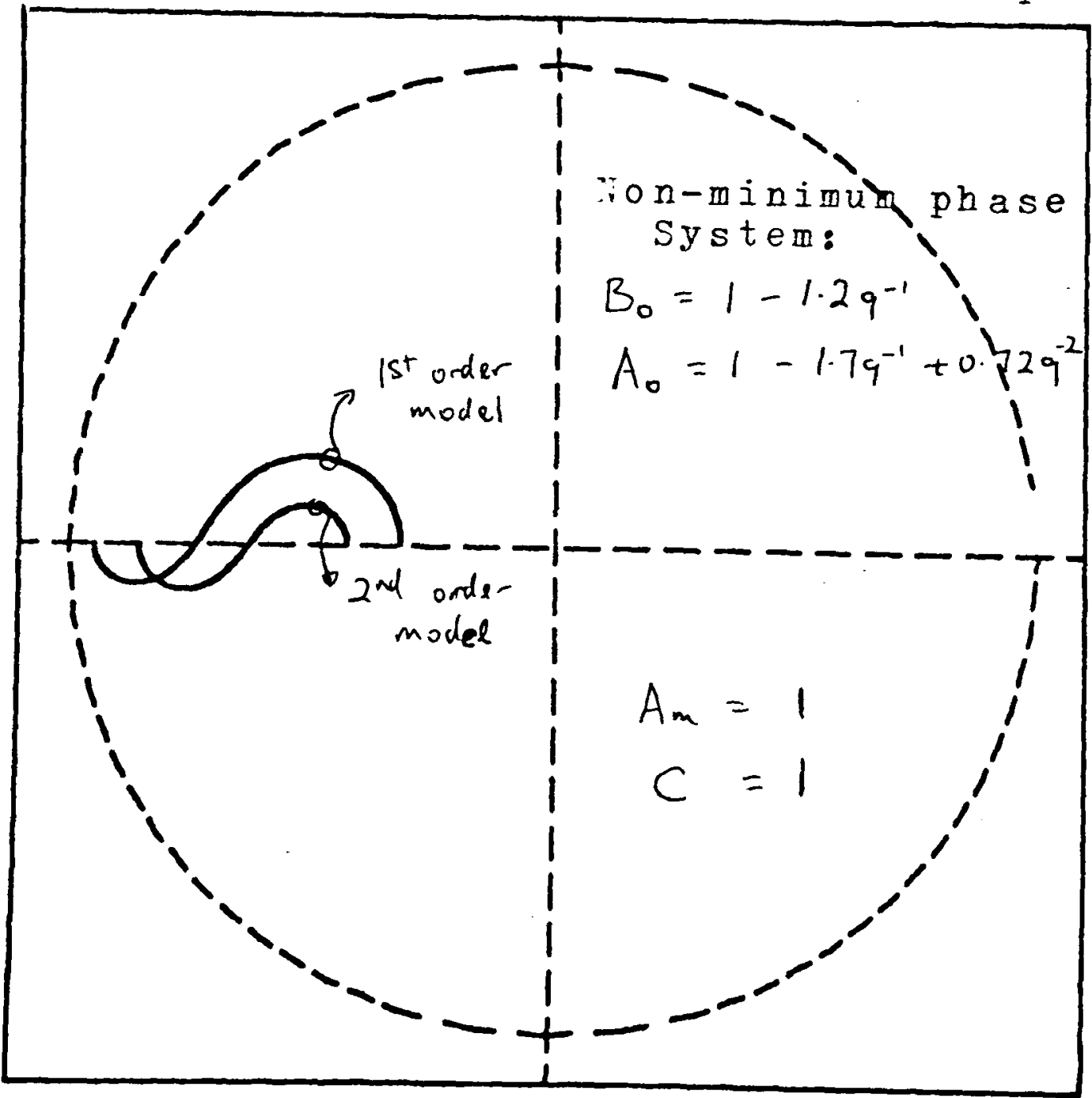

Fig. 3-15 Nyquist Loci for Varying Nominal order 
ROBUSTNF.SS OF THF, ADAPTIVF, CONTROTIT,F

\subsubsection{Discussion: Some other stability Work}

For the general explicit self-tuning algorithm described in section 3.6, there does not appear to be a robustness analysis yet. However the stability of a linear plant, controlied by a full order explicit self-tuner has been studied by a number of authors.

In Fuchs[36], sufficient conditions are specified for the estimator and the control law so that the closed loop system is stable. The disturbance is assumed to be an uncorrelated zero mean sequence. It is also assumed that at every time instant, the identified plant is uniformly stabilisable i.e. the common roots of $\hat{A}(t)$ and $\hat{B}(t)$ are stable roots. This is important because as shown above, the control law controls the identifjed model. Common roots are uncontrollable and hence must be stable. Another restrictive condition is that both the parameter estimates and the control law must be asymptotically time-invariant. This compares poorly with the implicit self-tuner, where neither estimator nor control law is required to be asymptotically time-invariant for stability.

In Samson and Fuchs[75], the conditions specified in Fuchs[36] are verified for a disturbance free system, using a state feedback control law and a scalar gain type of estimator (discussed later in the chapter scalar gain estimators are generally slower than matrix gain estimators).

Goodwin and $\sin [48]$ and De I,arminat[26],[27] have also developed stability proofs for plants which are disturbance free. They too base their proof on showing that the estimator and control law are asymptotically time-invariant and the asymptotic system is stable. 


\section{7 O SF.QUTFNCF}

In the robust stability analysis presented so far, the gain of the functional relationship which represents the estimator, ${ }_{\text {cp }}^{\prime}$ is a function of $\sigma_{0}$, where

$$
\begin{aligned}
& \sigma(t) \cong x^{T}(t) s^{-1}(t) x(t) \\
& \sigma_{0} \cong \sup _{t>t_{0}} \sigma(t)
\end{aligned}
$$

(NB: In this section, we will for notational convenience, allow $x$ to represent $x$ or $x_{0}$, according to the context)

To get the best robustness results, the gain of $B_{c p}$ should be as small as possible, which impljes small $\sigma_{0}$. In this section, some bounds on the sequence, $\{o(t)\}$ will be examined. Not surprisingly, the results require that the data in the data vector are "persistently exciting" in a well-defined and non-restrictive sense. However, this is still an a priori assumption in the closed loop control context. In Chapter 5 , simulation examples suggest that it is a valid assumption.

\section{Temma I}

Assume that the data vector, $x(t)$, is uniformly bounded. then $0<\sigma(t)<1$. 
ROBUSTNFSS OF THF, ADAPTIVF. CONTROTI.T.R

In Appendix B.5. This lemma has previously been presented in Gawthrop $[40]$

口

Remarks

Note that in Lemma 1 , no assumptions are made about the nature of the data vector sequence, other than that it must be $1_{\infty}$ bounded.

In Iemma 2 below, a 'persistently exciting' type of condition is imposed on the data vector sequence to ensure a tighter asymptotic limi.t on $\sigma(t)$.

\section{I.emma 2}

Assume that $x(t)$ is uniformly bounded.

If $\beta=$ forgetting factor $=1$ and

a) $\frac{S(N)}{N}$ is a uniformly bounded sequence with a finite limit point

b) $\lim _{t_{0} \rightarrow \infty} \inf _{N}>t_{0} \frac{s(N)}{N}>0$

then $\lim _{N \rightarrow \infty} \sigma(N) \rightarrow 0$

Proof See Gawthrop[40] 
Note that $x$ is not $1_{2}$ bounded if $\lim _{N \rightarrow \infty} S(N) / N>0$

The conditions of Iemma 2 would be met if for instance, the setpoint has a non-zero mean and the output $y(t)$ tracks the setpoint. This was pointed out in eg. Gawthrop[40] where the $x$ vector is decomposed into a sum of elements of orthogonal subspaces.

$$
x=x_{1}+x_{2}
$$

Then

$$
\begin{aligned}
& x^{T}(t) x(t)=\left[x_{1}{ }^{T}(t)+x_{2}{ }^{T}(t)\right]\left[x_{1}(t)+x_{2}(t)\right] \\
& =x_{1}^{T}(t) x_{1}(t)+x_{2}{ }^{T}(t) x_{2}(t)
\end{aligned}
$$

Bence either $x_{1}$ or $x_{2}$ not being $1_{2}$ bounded will suffice. An example of such a decomposition would be when $x_{1}$ (of the same dimension as $x$ ) contains only the output, $y$ terms of $x$, with zeros where the input, $u$ terms would be and $x_{2}$ similarly contains only u terms. Recently, Anderson and Johnson[4] have also formulated similar but more general conditions.

Note that if $x$

is an $I_{2}$ sequence and $\beta=$ constant $<1$, then $s$ would be an $l_{1}$ sequence. To see this observe from Eqn. 94 that

$$
s(t)=\sum^{t} \beta^{t-i} x^{T}(i) x(i)
$$

The cauchy product i.e.

$$
\sum^{\infty} s=\sum^{\infty} \beta^{i} \sum^{\infty} x^{T} x \text { if } \sum^{\infty} x^{T} x \text { exists. }
$$

Bence $\lim _{t \rightarrow \infty} g(t) \rightarrow 0$, which implies that the eigenvalues of $s^{-1} \rightarrow \infty$. Bowever, as $\lim _{t \rightarrow \infty} x^{T} x \rightarrow 0$ too, it is difficult to determine the behaviour of $\sigma$. Since $s(t)$ in Eqn. 94 is a scalar written in a recursive form, Eqn. 93 can easily be implemented if desired. Note that the properties of the estimator discussed in section 3.1 do not require that 
B i.s a constant.

In both Lemmas above, no restriction has been placed on the delay, $k$, which need not be 1 . Note that in the robust stability analysis, uniform boundedness is shown via the $1_{2 e}$ stability of the exponentially weighted associate system. $1_{2}$ stability of the associate system, however does not necessarily imply $1_{2}$ stability of the error feedback systems, i.e. in proving that the error feedback system is $1_{\infty}$ input-output stable, we have not implied that the system is also $1_{2}$ stable. Hence the conditions of Iemma 2 are not inconsistent. However, there is a technical problem in that for $I_{\infty}$ stability, $\beta$ < but Iemma $2 a$ requires that $\beta=1$.

Both Lemmas are deficient in that we really require that $\sigma(t)$ is less than some small constant, $\sigma_{0}$, for all time instants greater than a finite $t_{0}$. Neither Lemmas 1 or 2 satisfy this. It is instructive to note some situations when $\sigma(t)$ would tend to increase. From the proof of Lemma la

$$
\sigma(t)=\sigma_{1}(t)\left[\beta+\sigma_{1}(t)\right]^{-1}
$$

where $\sigma_{1}(t)=x^{T}(t) s^{-1}(t-1) x(t)$

An unmeasured and large (over unit time interval) load disturbance would lead to a large $\sigma_{1}$ and hence $\sigma(t)$. One solution to this problem of $\sigma$ spikes is to switch off the estimator for a short period. Another case of $\sigma(t)$ increasing would be when $s^{-1}(t)$ increases eg. when $\beta<1$ and the data vector is 'quiet' in the sense that $y_{0}(t) \rightarrow y_{0}(t-x)$ and $u_{0}(t) \rightarrow u_{0}(t-1)$. Then $\sigma_{1}(t) \rightarrow \sigma(t-1)$, which implies that $\sigma(t)>\sigma(t-1)$ if $1-\beta>\sigma(t-1)$. A possible remedy to this is the variable forgetting factor method of Fortesque et al[34] where $\beta \rightarrow 1$ when the data vector is 'quiet'. 


\subsection{ANOTHFR FSTIMATOR}

The estimator described by F.qns. 1 to 3 (Section 3.1 ) has a matrix gain factor in the parameter update equation. This appears to be the most favoured type of estimator in practical applications. Another estimator that is popularly studied in adaptive control has a scalar gain i.e.

The parameter updating is done as:

$\hat{\theta}(t)=\hat{\theta}(t-1)+s^{-1}(t-k) x_{0}(t-k) \hat{e}_{1}(t)$

and the gain update is

${ }^{*} s(t)=\beta s(t-1)+x_{0}{ }^{T}(t) x_{0}(t)$

$s(0)=s_{0}>0$

This is sometimes called a stochastic approximation estimator. Many of the first proofs of stability for self-tuning controllers were obtained using this type of estimator eg. Goodwin's. Hence it is of interest to extend the robustness results for the matrix gain estimator to this case. This is done only for the case of the implicit self-tuning controllex. The results show that there is a theoretical advantage of this estimator in that robust stability results are obtained which do not depend on an a priori assumption of small $\sigma_{0}$. The method of analysis is identical to that of the preceding sections. Unlike Goodwin, the only restriction on the disturbances is that they must be bounded but the results are a weakex form of stability.

As in Iremma 1, Section 3.7, a simple modification to ensure that $\mathbf{s}(t)$ is a non-decreasing scalar is achieved by selecting $\beta(t)$ such that

$$
B(t)>1-s^{-1}(t-1) x_{0}^{T}(t) x_{0}(t)
$$

The usefulness of this modification will be apparent in Lemma 2 .

* Comparing with Eqn $3^{-2}$ it can be shown that $s(t)=$ trace $[s(t)]$ where $s(t)$ is the gain factor in the matrix gain estimator. 
ROBUSTNF.SS OF THF. ADAPTIVF. CONTROT.IAER

If a parameter $\sigma_{s}(t) \bullet x_{0}^{T}(t) x_{0}(t) s^{-1}(t)$ is defined as a scalar version of $o(t)$ in the matrix gain case (cf section 3.7 ), the robust stabijity results in section 3.4 are equaljy valjd when this estimator j.s used with $o_{0}$ replaced by sup $t_{t>t_{0}} o_{s}(t)$.

An alternative is to define an error feedback system with weighted error signals. Theorem 1 below uses the small gain approach to show robust stability. This method was also used by Gawthrop[41] to show ${ }_{2}$ stability of the linear full order case via a passivity approach.

The scalar gain of Eqn. 103 is used to form the weighting sequence, $\mathrm{s}^{-1 / 2}$ giving the error feedback system of Fig. 3-16 below.

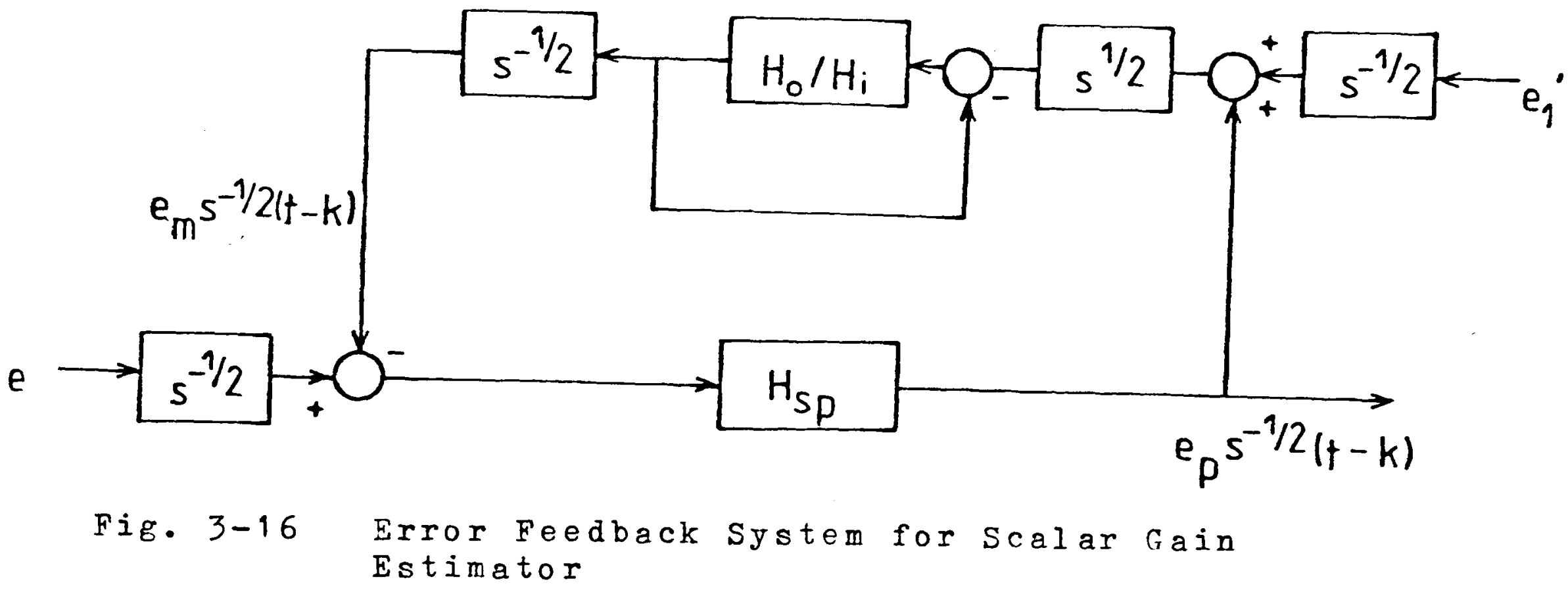

Fig. 3-16 can be obtained from those of section 3.4 by a simple $100 p$ transformation. It is also assumed that delay $k$ is minimal and that $Q=0$ i.e. the plant is assumed to be stably invertible. Iremma $I$ below, corresponding to Iemma 1 in section 3.1 , states the gain of the functional relationship $B_{s p}$ which represents the estimator in this error feedback system. Iemma 2 is a technical result needed later in the section. 
ROBUSTNF.SS OF THF. ADAPTTVF, CONTROIT,F.R

\section{T,emma $].$}

The gain of $\mathrm{H}_{\text {sp }}=1$ i.e.

$$
\begin{aligned}
& \sum_{j}^{N} e_{p}^{2}(t) s^{-1}(t-k) \leqslant \sum\left[e_{p}^{N}(t)+\hat{e}_{1}(t)\right]^{2} s^{-1}(t-k)+\text { constant } \\
& \forall N>t_{0}
\end{aligned}
$$

Proof In Appendix B.6.

\section{Lemma 2}

Let $I$ be a bounded (not necessarily linear) operator, with gain $\gamma$, mapping $I_{2}$ to $1_{2}$. Consider the operator $s^{-1 / 2} I_{1} s^{1 / 2}$ where $s$ is a non-decreasing scalar sequence. Then $L_{\Lambda}^{\prime} s^{-1 / 2} \mathrm{Isg}^{1 / 2}$ also has gain $\gamma$, in the sense that

$$
\sum\left[I_{1}[x]\right]^{2} \leqslant y^{2} \sum^{N} x^{2} \quad \forall N>t_{0}
$$

Proof In Appendix B.6.

Applying the small gain theorem to Fig. 3-16, it is obvious that the $I_{2}$ gain of $\mathrm{H}[1+\mathrm{H}]^{-1}<I$ for robust stability, where $\mathrm{H}=\mathrm{B}_{0}$ in $\mathrm{Fig}$. 3-7 for the output error case and $H=H_{i}$ in $\mathrm{Pig} .3-8$, for the input error case. It can easily be shown [eg. via the $M$ circle plots of section 2.5 ] that this is equivalent to requiring that $\left(H+\frac{1}{2}\right)$ is a passive operator in the sense that

$$
\sum^{n}\left[y x+\frac{1}{2} x^{2}\right]>0
$$

where $y=B(x)$

When considering the linear higher case, $\mathrm{H}_{0}=\mathrm{H}_{i}$ and 
ROBUSTNF.SS OF THF. ADAPTIVF. CONTROT,T.F.R

$$
H[1+H]^{-1}=\frac{E\left(A_{0} B-B_{0} A\right)}{P\left(B_{0}+E\left(A_{0} B-B_{0} A\right)\right.}
$$

As $B_{0}$. $A_{0}$ will not usually be known, the gain of Fqn. 107 is difficult to evaluate directly. Passivity of $\mathrm{H}+\frac{1}{2}$ merely requires that the Nyquist locus of $H\left(q^{-1}\right)$ lie in the half plane to the right of $\left(-\frac{1}{2}, 0\right)$ i.e.

$$
\operatorname{Re}\left\{\frac{B F}{P C}\left[\frac{A}{B} 0-\frac{A}{B}\right]\right\}>-\frac{1}{2}: \forall q=e^{j \theta}, \theta \in[0,2 \pi]
$$

It is assumed that while $B_{0}, A_{0}$ may not be known, it is possible to get an estimate of the frequency response of the difference between true and nominal plants.

When $H$ is nonlinear, the passivity condition is less useful because in general, a composition of passive operators is not necessarily passive. A sufficient but rather restrictive condition for $\mathrm{H}[1+\mathrm{H}]^{-1}$ to have ${ }_{2}$ gain < 1 is when $B$ has gain $<\frac{1}{2}$. However if we assume that the $A\left(q^{-1}\right), B\left(q^{-1}\right)$ polynomials represent known linear dynamics (unknown linear dynamics can be left in $M_{i}$ : cf. Chapter 2 ), then we can choose

$$
P=\frac{F A}{C}
$$

Bence $\mathbf{H}_{0}=\mathbf{P}\left(\mathbf{M}_{1}^{-1}-1\right) \mathbf{P}^{-1}$ in the output error case and

$$
\mathrm{B}[1+\mathrm{B}]^{-1}=\mathrm{P}\left[1-\mathrm{M}_{1}\right] \mathrm{P}^{-1} \text {. }
$$

Using Lemma 1 of Section 3.4.1, if $M_{1}$ is continuously Frechet diffferentiable, then gain $\left(\mathrm{H}[1+\mathrm{H}]^{-1}\right\}=M-\mathrm{M}_{1} \|_{\Delta}$

The small gain theorem applied to Fig. 3-16, together with the relationship between the error quantities, Eqn. 2-33, says that if es ${ }^{-1 / 2}$ and $e_{1} \cdot s^{-1 / 2}$ are in $l_{2}$ then so is $\hat{e}_{1}(t) s^{-1 / 2}, i . e$.

$$
\sum_{1}^{\infty} \hat{e}_{1}^{2}(t) s^{-1}(t-k)<\infty
$$

However as $g$ is a non-decreasing sequence, this is weaker than the corresponding result for the matrix gain case, where $\sum_{e}^{\infty} \hat{e}_{1}^{2}(t)<\infty$. If $\beta$ is selected as in Eqn. 105 to ensure that $s(t)$ is a strictly increasing 
ROBUSTNFSS OF THF. ADAPTIVF. CONTROTITFR

sequence, then Kronecker's Irema gives that

$$
\lim _{N \rightarrow \infty} \quad \frac{1}{8(N)} \sum_{1}^{N} \hat{e}_{1}^{2}=0
$$

At this point, note that in Goodwin[49], a result similar to Eqn. 1.09 i.s arrived at via a martingale approach, assuming that the controller is of full order and the disturbance has a specific stochastic structure. If the plant is assumed to be stably invertible, ( $Q$ would not be zero otherwise), then a development from Eqn. 109 similar to Goodwin's[49] yields that

$$
\lim _{N \rightarrow \infty} \frac{1}{N} \sum_{1}^{N} \hat{e}_{1}^{2}(t)=0
$$

Theorem 1 bejow summarises the result.

\section{Theorem I.}

Iret the self-tuning adaptive control law of section 2.3.1, with $Q=0$ and the scalar gain estimator (Eqns. 102 to 105) control a stably invertible plant of minimal delay $(k=1)$, modelled as in section 2.2.

The corresponding error feedback system is in Fig. 3-16 and

$$
\lim _{N \rightarrow \infty} \frac{1}{N} \sum^{N}[\Phi(t)-\operatorname{Rw}(t-1)]^{2}=0
$$

if. $e, e_{1} \cdot \epsilon I_{2} ; P^{-1}, c^{-1}$ are stable and EITtHer

a) the plant is linear but the controller is of incorrect order and there exists $A$, stable $B$ such that $H+\frac{1}{2}$ is passive.

OR

b) $M_{i}$ is nonlinear, time-invariant and there exists a scalar $y$ such that

$\operatorname{gain}\left\{\mathrm{B}[1+\mathrm{H}]^{-1}\right\}<1$

where $B=\frac{F A}{C}\left(Y M_{1}^{-1}-1\right) \frac{1}{P}$ in the output error case 
ROBUSTNF.SS OF THF, ADAPTIVE, CONTROTITA.R

$$
\text { and } H=\frac{A E}{P C} \frac{B P}{A}\left(\gamma M_{2}^{-1}-1\right) \frac{A}{P R} \text { in the input error case. }
$$

Proof In Appendix B.6.

Rema rikg

Unlike the results for the matrix gain estimator, Theorem 1 does not need to assume that $\sigma_{0}<1$. However Theorem 1 implies that the scalar gain estimator is less robust than the matrix gain estimator in the sense that while requiring the inputs, $e, e_{1}$, to be of finite, energy (i.e. $\left.\epsilon I_{2}\right), \hat{e}_{1}(t)$ is only of finite power. Requiring the inputs to be of finite energy is restrictive. For example, if the inputs have a non-zero mean level, they are not finite energy.

The result of Eqn. Ill has been obtained without any assumption on the structure of the disturbance and unlike Goodwin's[49], for a plant which may be nonlinear or of large order. If the plant is linear, then Theorem 1 requires that $B_{0}$ must have stable roots. If it is nonlinear, we assume, as before that the linear subsystem is minimum phase and that $\mathbf{M}^{-1}$ exists and is bounded.

It is interesting to note that unlike the matrix gain case, it is possible to show that the estimator is asymptotically time-invariant. To see this, note that iff $\hat{e}_{1} s^{-1 / \eta^{\prime}} \in \tau_{2}$ '

$$
\lim _{t \rightarrow \infty} \hat{e}_{1}^{2}(t) s^{-1}(t-k)=0
$$

From Eqn. 102, and noting that $s^{-1}(t-k) x^{T}(t-k) X(t-k)<1$

$$
\begin{gathered}
{[\hat{\theta}(t)-\hat{\theta}(t-1)]^{T}[\hat{\theta}(t)-\hat{\theta}(t-1)]=s^{-2}(t-k) x^{T}(t-k) x(t-k) \hat{e}_{1}{ }^{2}(t)} \\
<s^{-1}(t-k) \hat{e}_{1}{ }^{2}(t)
\end{gathered}
$$

Bence $\lim _{t \rightarrow \infty}[\hat{\theta}(t)-\hat{\theta}(t-1)]^{T}[\hat{\theta}(t)-\hat{\theta}(t-1)]=0$ 
ROBUSTNESS OF THE ADAPTIVE CONTROLLER

It is also possible to apply the exponential weighting techniques of the earlier sections to show that if $e, e_{1}(t)$ are uniformly bounded, then so is $\hat{e}_{1}(t) s^{-1 / 2}(t-k)$. However this would be considerably weaker than the matrix gain case.

To ensure that $s(t)$ is strictly increasing, it may be necessary for $\beta$, I eg. when $\mathrm{x}_{0}^{\mathrm{T}}(t) \mathrm{x}_{0}(t) \mathrm{s}^{-1}(t-1) \rightarrow 0$. Unlike the matrix gain case, this is permissible as the relationship of Lemma 1 is independent of $\beta$.

To summarise: the use of scalar gain estimators gives neat theoretical results compared to matrix gain estimators. There is no $\sigma_{0}$ dependence and the estimator is asymptotically time-invariant. However the results indicate that they are less robust than matrix gain algorithms and this is borne out by the preference for matrix gain estimators in practical applications.

\subsubsection{Examples}

\section{Example 2 of Section 3.4 .2 (Continued)}

Consider Example 2 of section 3.4.2. If a scalar gain estimator is used then $\mathrm{H}_{\mathrm{O}}+\frac{1}{2}$ is required to be passive. Fig. 3-17 shows Nyquist plots of $\mathrm{B}_{0}$ for fractional delays of $0.3 \mathrm{~T}, 0.4 \mathrm{~T}, 0.5 \mathrm{~T} . \mathrm{All}$ have real parts > -0.5

Example 3 of section 3.4 .2 (Continued)

Consider Example 3 of Section 3.4.3. With a scalar gain estimator and minimum variance control $(P=1)$, it is not possible to find an $A, b$ such that $H_{0}+\frac{1}{2}$ is passive. When $P=2-q^{-1}$, it is possible. 
ROBUSTNF.SS OF THF. ADAPTIVF, CONTROTITER

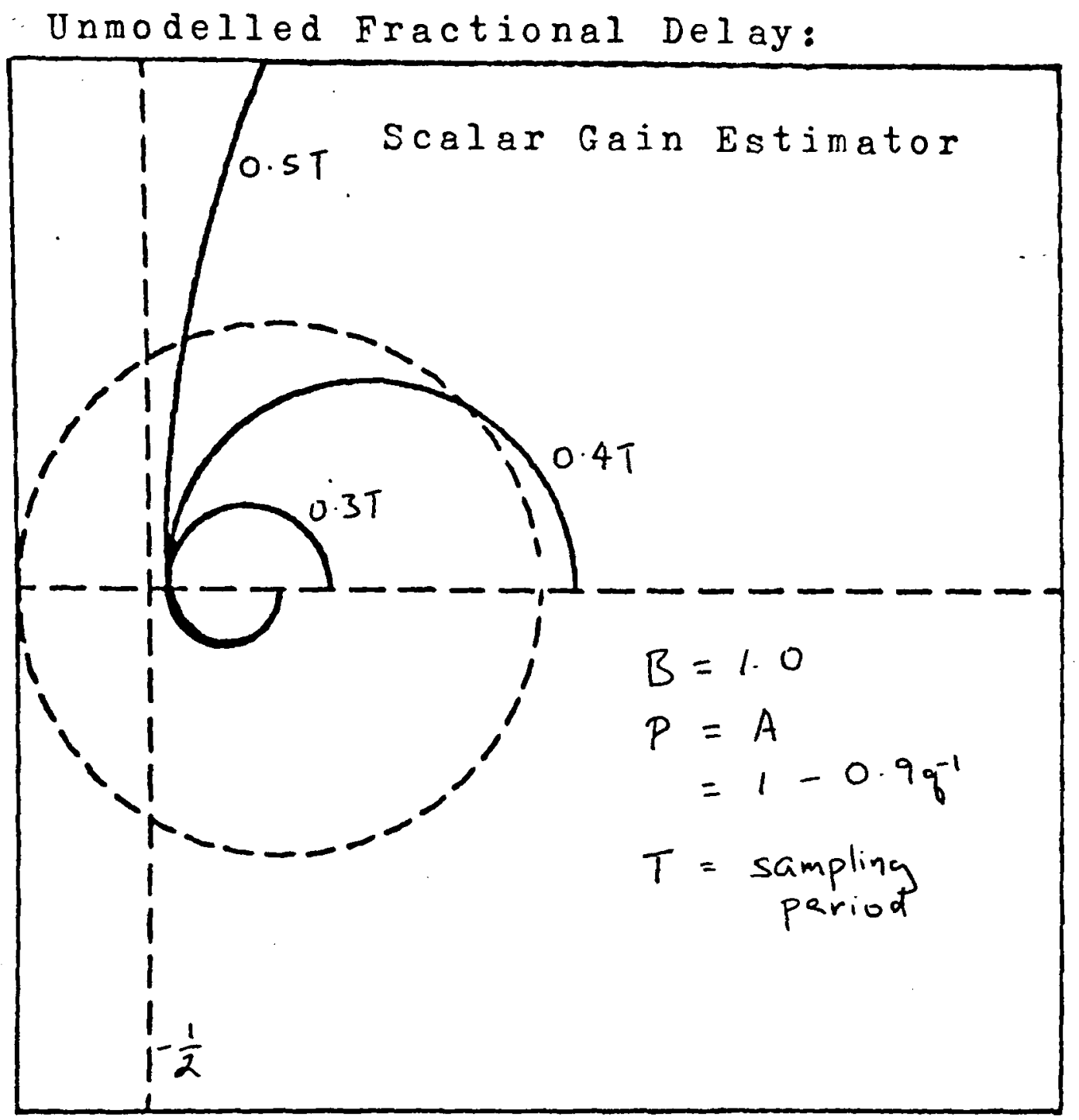

Fig. 3-17 Passivity of $\mathrm{H}_{0}+\frac{1}{2}$ (varying delay)

Fig. 3-18 shows an example of each case.

\subsection{DISCUSSION}

The robust stability results of sections $3.2,3.4$ and 3.6 are in terms of $l_{2}$ or $l_{\infty}$ stability. In section 3.4 for instance the input to the error feedback system, $e(t), e_{1}^{\prime}(t)$ are required to be in $l_{2}$ or $l_{\infty}$ and the results give that $\hat{e}_{1}(t)$ is in $l_{2}$ or $l_{\infty}$. If $\hat{e}_{1}(t)$ is only required to be such that

$$
\lim _{N \rightarrow \infty} \quad \frac{1}{N} \sum^{N} \hat{e}_{1}^{2}(t)=0
$$




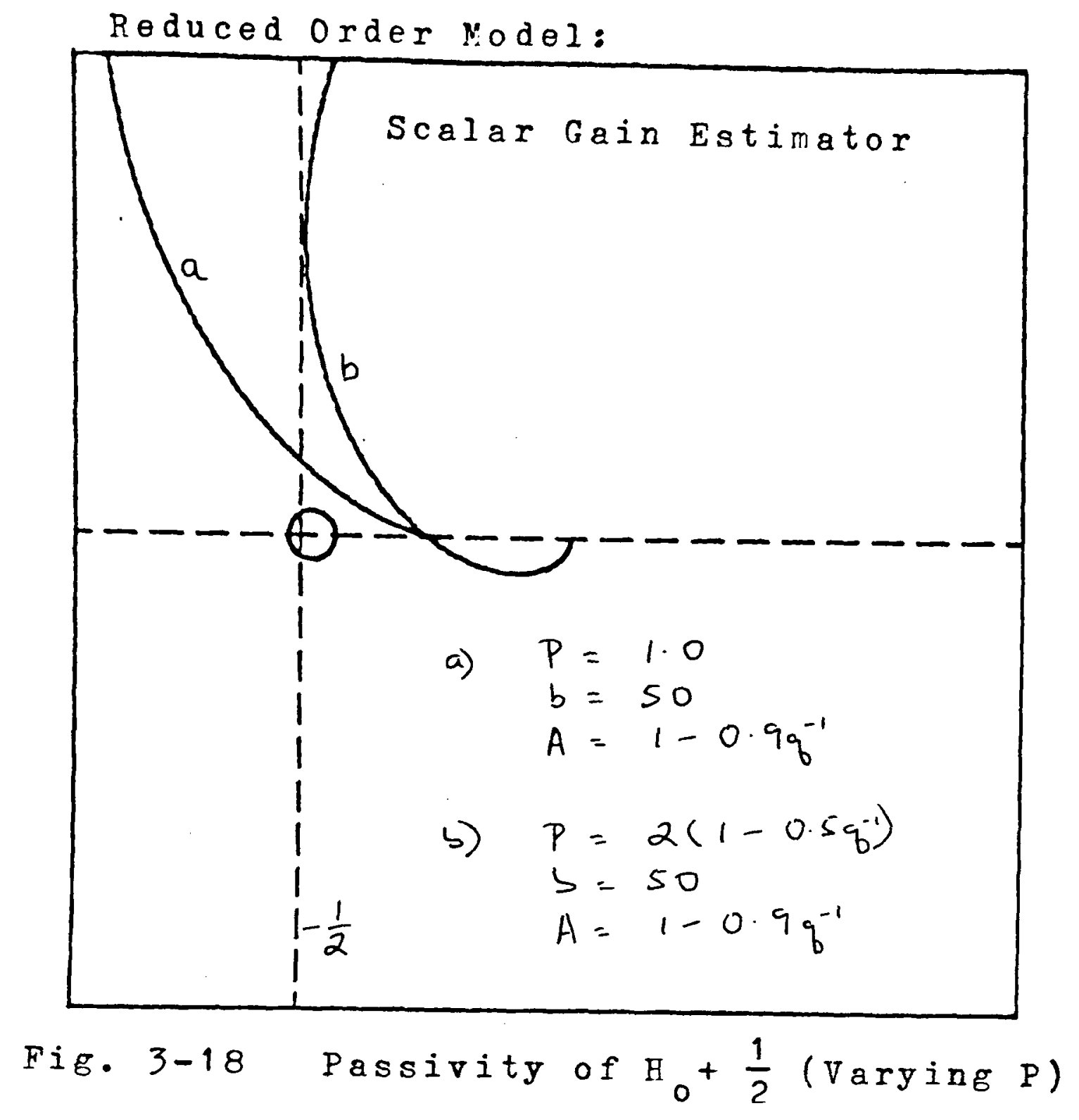

it can be shown, with the uge of remma 2. Section 3.8 that only $t^{-l / 2} e(t), t^{-1 / 2} e_{1} \cdot(t)$ are required to be in $z_{2}$, which is slightly weaker than $e(t), e_{1}^{\prime}(t) \in 1_{2}$.

J.erma 1

Consider the error feedback systems of sections 3.2, 3.4 and 3.6 . Then F.qn. 114 above is true if the inputs to the error feedback system, weighted by $t^{-1 / 2}$ are in $1_{2}$, in addition to the other conditions of the respective theorems. 
ROBUSTNF.SS OF THE, ADAPTIVF, CONTROTI.F.R

The robust stability results for self-tuning adaptive controljers have been obtained using standard input-output results. Some early work on reduced order adaptive controljers was also done by Goodwin and Ramadge[45]. There only a minimum variance type regulator was considered. Simulation results were presented to show that this was not robust.

Robustness to modelling errors is approached from another angle in Bitmead and Anderson[15] and Anderson and Johnson[4]. The former considers only the estimation problem, while the latter includes an adaptive control problem. In both papers the argument runs that the estimation and/or control scheme is unlikely to be robust unless the nominal closed loop system is shown to be exponentially asymptotically stable. In the latter, this was shown for a linear, minimum phase nominal system, with a scalar gain type estimator, under persistently exciting conditions on the setpoint. These results are strong in that the nominal system parameters are shown to converge exponentially fast. It is worth noting however, that the $I_{2}$ stability approach adopted in this thesis would include exponential stabjjoty under certain controllability and observability assumptions on the plant. see eg. Anderson [3], wi]lems[90].

In the case of the explicit self-tuner, the robustness results of this thesis do not go very far. This is not surprising in view of the fact that there are few results as yet of the stability of the explicit self-tuner when controlling a nominal linear plant of known order. In Kreisselmeir[53], a different form for an explicit adaptive state 
ROBUSTNF.SS OF THF, ADAPTIVF. CONTROTITFR

regulator i.s proposed. An adaptive observer is used together with a control law which is only asymptotically certainty equivalent (even in the case of known parameters, such a control law would only tend asymptotically to the desired control law). The closed loop system is shown to be locally exponentially stable in the Iyapunov sense, even in the presence of modelling exrors. As in this thesis, parameter convergence is not required. Furthermore, the results only require the existence of arbitrary equilibrium points satisfying certain conditions.

Unlike the non-adaptive case considered in Chapter 2, the results of this chapter are solely concerned with robust stability. It does not appear a simple matter to get robust disturbance attenuation results. Here it will only be noted that if the parameter estimates are asymptotically time-invariant, then the results of the non-adaptive case in Chapter 2 will be valid in the limit. 


\section{CHAPTER 4}

ROBUSTNESS OF THE PRACTICAI SELF-TUNER

\subsection{INTRODUCTION}

In Chapter 3, a simplified self-tuning controller was examined for robustness. This chapter examines the robustness of the clarke-Gawthrop self-tuner when some of the simplifying assumptions are removed.

In Section 4.1, the problem of showing robustness of the self-tuner when the delay is non-minimal $(k>1)$ is studied. Most of the early stability results for the stability of self-tuning controllers were valid only for $k=1$. However this is often an unrealistic assumption. Subsequent work has modified the original algorithms to cope with non-minimal delays eg. Goodwin's[49] multiple recursion algorithm. It turns out that while this algorithmic modification can be analysed with the methods of this thesis, the standard self-tuner can be similarly studied and hence there is no need for the modification.

A second assumption implicit in Chapters 2 and 3 is that the error feedback systems derived there have solutions. Section 4.2 considers a few cases when this is not justified. A fresh interpretation for the role of the design polynomial, $Q\left(q^{-1}\right)$ is offered. Note that there are many other interpretations for the role of $Q\left(q^{-1}\right)$ eg. clarke and 
ROBUSTNESS OF THE PRACTICAL SELF-TUNER

Gawthrop[19], Gawthrop[37], Morris and Nazer[69]. These will be reviewed in Chapter 5.

The third section of this chapter deals with the problem of removing offsets. This did not arise in Chapters 2 and 3 partly because it was assumed that all the disturbances had zero means. The practical solution to this problem is by no means settled. Many methods have been proposed and the contribution of this thesis is to examine some of them for robust stability only, when used in conjunction with the clarke-Gawthrop self-tuner.

\subsection{NON-MINIMAL DELAY}

In Chapter 3, it was usually assumed that the integer delay, $\mathbf{k}=1$. In most practical systems this will not be the case, due for instance to the presence of transport lags.

Non-minimal delay is a problem in the analysis of robust stability because the corresponding error feedback system is more difficult to For the purpore of analysis,
dexive. $\wedge$ There is a mismatch between the output of the estimator relationship, $\mathrm{H}_{\mathrm{CP}}$ (Cf Section 3.1 ) and the input to $\mathrm{H}_{0}$ or $\mathrm{H}_{i}$ i.e. the operator representing modelling errors. (See figures of section 3.3). From Eqns. 2-34, 2-35 for instance, the input to $\mathrm{H}_{0}$ in the output error case contains the $\mathbf{k}$ steps ahead estimated prediction error:

$$
\begin{aligned}
\hat{e}_{k}(t) & \equiv \Phi(t)-x_{0}^{T}(t-k) \hat{\theta}(t-k) \\
& =\Phi(t)-\hat{\Phi}_{k}(t)
\end{aligned}
$$

while the output of $H_{c p}$ ( Cf section 3.1) is the one step ahead estimated prediction exror: 
ROBUSTNESS OF THE PRACTICAL SELF-TUNER

$$
\begin{aligned}
\hat{e}_{1}(t) & \equiv \Phi(t)-x_{0}{ }^{T}(t-k) \hat{\theta}(t-1) \\
& =\Phi(t)-\hat{\Phi}_{1}(t)
\end{aligned}
$$

This mismatch arises because a realisable control law must be causal and the $k$ steps ahead prediction at time $t$ can only use data known at time $t$ i.e.

$$
\hat{\Phi}_{k}(t+k / t)+Q u(t)=R w(t)
$$

or in terms of the estimated prediction error

$$
\Phi(t+k)+Q u(t)=R w(t)+\hat{e}_{k}(t+k)
$$

while it is natural for the estimator to use the latest available data in the parameter update i.e.

$$
\hat{\theta}(t+1)=\hat{\theta}(t)+s^{-1}(t+1-k) x_{0}(t+1-k) \hat{e}_{1}(t+1)
$$

This is a problem not peculiar to this method of analysis. In Goodwin et al[49],[47], an algorithm was proposed to overcome this problem, which effectively consists of $k$ (integer delay) interlaced estimators (scalar gain type). In the rest of this section, it will be shown that while Goodwin's algorithm fits the analysis of this thesis, the modification is not really necessary.

\subsubsection{Multiple Recursion or Interlacing}

In Goodwin[49], a scalar gain estimator is proposed as:

$$
\begin{aligned}
& \hat{\theta}(t)=\hat{\theta}(t-k)+s^{-1}(t-k) x_{0}(t-k) \hat{e}_{k}(t) \\
& s(t)=s(t-1)+x_{0}^{T}(t) x_{0}(t)
\end{aligned}
$$

The main difference from the estimator described in section 3.8 is that 
at $t$ ime $t$, the parameter update uses the estimated parameters and data of time t-k samples ago. (CE Eqn. 3-102).

Define a new parameter error as:

$$
e_{p}^{k} \equiv x_{0}^{T}(t-k) \dot{\theta}(t-k)
$$

and note that the relationship between the error quantities becomes

$$
\begin{aligned}
\hat{e}_{1}+e_{p} & =e_{p}^{k}+\hat{e}_{k} \\
& =e(t)-e_{m}(t)
\end{aligned}
$$

Let $\mathrm{H}_{\mathrm{sp}}^{\mathrm{k}}$ be the functional relationship between $e_{p}^{k}(t) \mathbf{s}^{-1 / 2}(t-k)$ and $\left[\hat{e}_{k}(t)+e_{p}^{k}(t)\right] s^{-1 / 2}(t-k)$, where $s^{-1 / 2}$ is a monotonically non-increasing weighting sequence (obvious from Eqn. 6 above). The gain of this relationship is given by

\section{$\underline{\text { Lemma } 1}$}

The gain of $\mathrm{B}_{\mathrm{sp}}^{\mathrm{k}}=1$ i.e.

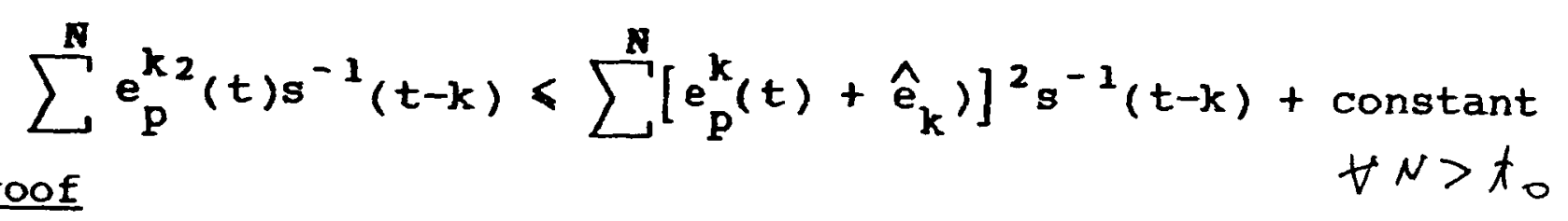

Proof

Similar to Lemma 1 , Section 3.8

口

The error feedback system corresponding to this estimator is then the same as Fig. 3-16, with $\mathrm{H}_{\mathrm{sp}}$ replaced by $\mathrm{H}_{\mathrm{sp}}^{\mathrm{k}}$, $e_{p}$ by $\mathrm{e}_{\mathrm{p}}^{\mathrm{k}}$ and $\hat{\mathrm{e}}_{1}$ by $\hat{e}_{k}$. For robust stability, we thus have: 
ROBUSTNESS OF THE PRACTICAL SELF-TUNER

\section{Theorem 1}

Theorem 1 of section 3.8, for the robust stability of the implicit self-tuning controller with a scalar gain estimator, is also valid when $k \neq 1$, provided that the estimator of Eqns. 5 and 6 above is used instead of Eqns. 3-102, 3-103.

A modification similar to Eqns. 5, 6 can also be done for the matrix gain estimator

$$
\begin{aligned}
& \hat{\theta}(t)=\hat{\theta}(t-k)+s^{-1}(t-k) x_{0}(t-k) \hat{e}_{k}(t) \\
& s(t-k)=s(t-2 k)+x_{0}(t-k) x_{0}^{T}(t-k) \text { :cf section } 3.1 \\
& s(0), s(1), \ldots, s(k-1)>0
\end{aligned}
$$

Let $\mathbf{B}_{c p}^{\mathbf{k}}$ be the functional relationship between $\hat{e}_{k}$ and $\hat{e}_{k}+e_{p}^{k}$. Corresponding to Lemma 1 of section 3.1, we have

\section{Lemma 2}

$$
\begin{aligned}
& \text { The gain of } \mathrm{B}_{\mathrm{Cp}}^{\mathrm{k}} \text { is }\left(1-\sigma_{0}\right)^{-1 / 2} \text { i.e. } \\
& \sum^{\mathrm{N}} \hat{\mathrm{e}}_{\mathrm{k}}^{2}(t)<\left(1-\sigma_{0}\right)^{-1} \sum^{N}\left[\hat{e}_{k}+e_{\mathrm{p}}^{\mathrm{k}}\right]^{2}+\operatorname{constant}(\mathrm{bias}) \quad \forall N>t_{0}
\end{aligned}
$$

\section{Proof}

In Appendix C.I

Knowing the gain of the estimator relationship, we have for robust stability: 


\section{Theorem 2}

When $k \neq 1$, the results of sections $3.4,3.5$ and 3.6 are also valid if the estimator characterised by $H_{c p}$ is replaced by the estimator described here i.e. $\mathbf{H}_{c p}^{\mathbf{k}}$.

\section{$\underline{\text { Proof }}$}

The output of $\mathrm{B}_{c p^{\prime}}^{\mathrm{k}}, \hat{\mathrm{e}}_{k}(t)$ is one input to $\mathrm{H}_{0}$ or $\mathrm{H}_{i}(F i g .3-5$ or $3-6)$. Lemma 2 shows that $\mathrm{H}_{\mathrm{Cp}}^{\mathrm{k}}$ has the same gain as $\mathrm{H}_{\mathrm{CP}}$. Hence the result follows. $\square$

The estimators characterised by Lemmas 1 and 2 fit into the analysis because they use delayed data, rather than the latest available data i.e. at time $t, \hat{\theta}(t-k)$ instead of $\hat{\theta}(t-1)$ is used in the parameter update. The modifications are artificial in that they are motivated by the limits of analysis rather than by practice. In the next subsection, the estimator represented by $B_{c p}$ is used even when $k \neq 1$, and a functional relationship is shown between $\hat{e}_{k}(t)$ and $\hat{e}_{1}(t)$.

\subsubsection{Standard Matrix Gain Estimator: $\mathbf{k} \neq 1$}

In the case of non-minimal delay, we need a new estimator relationship for the error feedback model. In this section, the estimator is represented as the composition of two operators. Let $H_{e}$ denote the functional relationship between $\hat{e}_{k}(t)$ and $\hat{e}_{1}(t)$ and $H_{e}^{j}$ the relationship between $\left(\hat{e}_{j}-\hat{e}_{j-1}\right)$ and $\hat{e}_{1}(t)$. Fig. 4-1 below represents $B_{e}$ in terms of $\mathrm{B}_{\mathrm{e}}^{\mathrm{j}}$.

Let the gain of $\mathrm{B}_{e}^{j}$ be $\gamma_{j}$ and the gain of $\mathrm{B}_{e}$ be $\gamma_{e}$. 


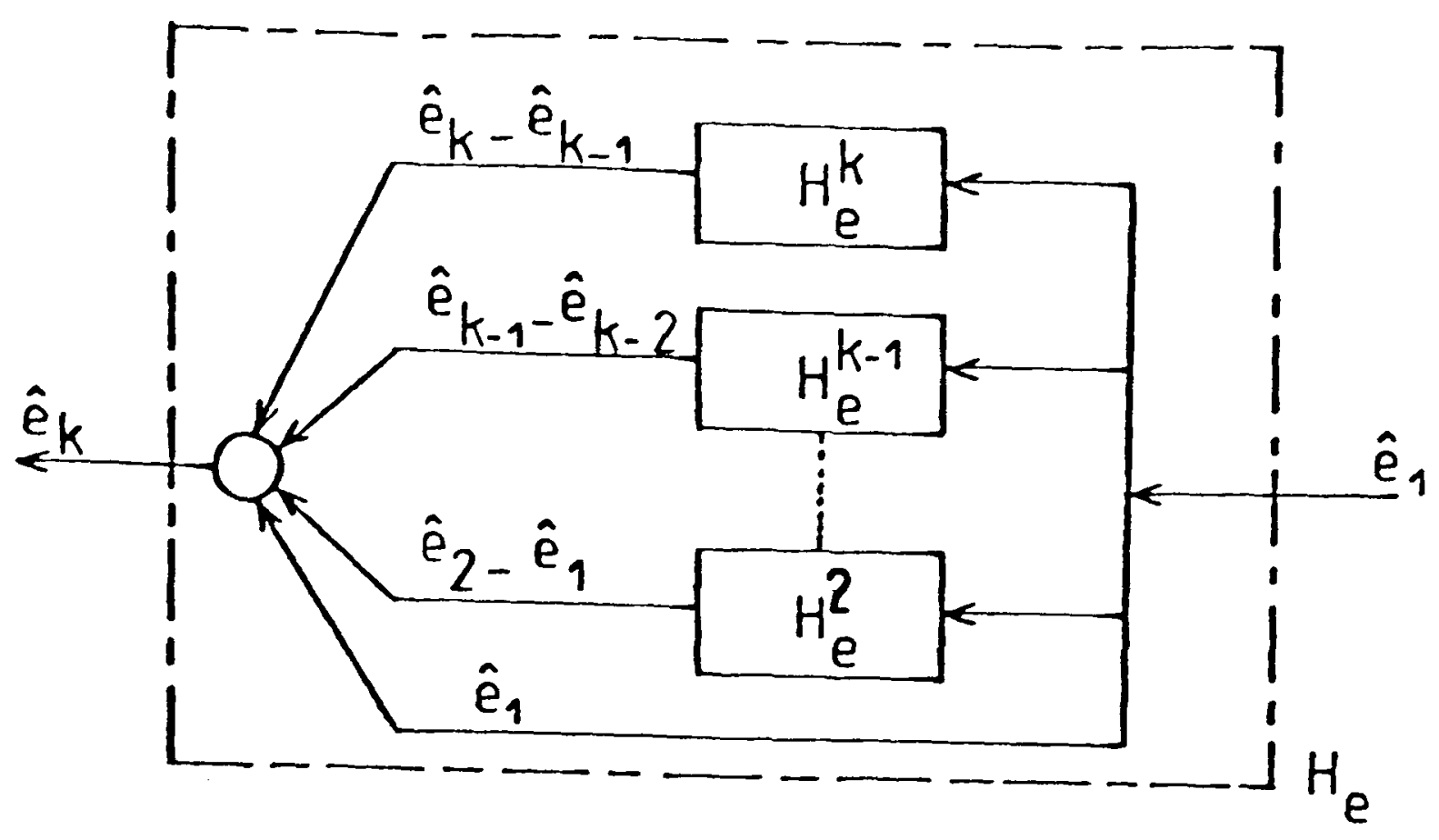

Fig. 4-1. Relationship between error quantities

$$
\gamma_{e} \leqslant 1+\gamma_{2}+\ldots+\gamma_{k}
$$

Define:

$$
\left.\sigma_{2}(t, i)=x_{0}^{T}(t+i) s^{-1}(t) x_{\delta} t+i\right)
$$

then

$$
\sigma(t)=\sigma_{2}(t, 0)
$$

Lemma 1

The gain of $\mathrm{B}_{e}^{j}$ is $\gamma_{j}$

where

$$
\begin{aligned}
y_{j}^{2} & =\sigma_{0} \sigma_{2}^{s}(j-1) \quad: j>2 \\
\sigma_{0} & =\sup _{t>t} \sigma(t) \\
\sigma_{2}^{s}(i) & =\sup _{t>t} \sigma_{2}(t, i) \quad: i>0 \\
& \leq \frac{\beta \sigma_{0}}{1-\sigma_{0}}\left[\beta^{i-1}+\beta^{i-2} \sigma_{2}^{s}(1)+\ldots+\sigma_{2}^{s}(i-1)\right]
\end{aligned}
$$

$\beta$ is the forgetting factor 
ROBUSTNESS OF THE PRACTICAL SELF-TUNER

Proof

In Appendix C.2

Fig. 4-1 and Lemma 1 permit a characterisation of the relationship between $\hat{e}_{k}(t)$ and $\left[\hat{e}_{l}(t)+e_{p}(t)\right]$ as

$$
\hat{e}_{k}(t)=H_{e} H_{c p}\left\{\hat{e}_{1}+e_{p}(t)\right\}
$$

From Eqn. 12 and Lemma $I$ of Section 3.1 , the gain of $\mathrm{B}_{\mathrm{e}} \mathrm{CP}_{\mathrm{CP}}$ is $\gamma_{\mathrm{e}}\left(1-\sigma_{0}\right)^{-1 / 2}$

Bence, if delay $k \neq 1$, but the standard matrix gain estimator of Eqns. 3-1 to $3-3$ is still used, the error feedback systems of Sections 3.3 to 3.6 are unchanged if $\mathrm{H}_{\mathrm{e}} \mathrm{B}_{\mathrm{cp}}$ replaces $\mathrm{B}_{\mathrm{cp}}$. Theorem 1 below states the obvious consequence.

\section{Theorem 1}

When $k \neq 1$, the results of sections $3.4,3.5$ and 3.6 are also valid if

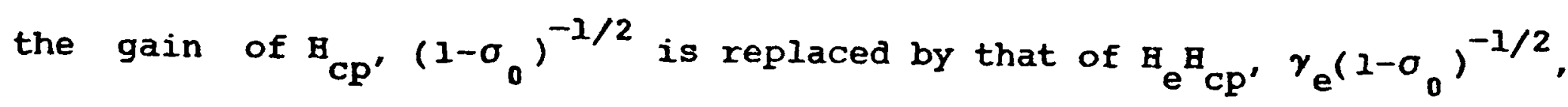
where $\gamma_{e}$ can be evaluated by Eqn. 12 and Lemma 1 above.

\section{$\underline{\text { Remarkg }}$}

Note that $\gamma_{e}>1$. Each extra integer delay increases the effective gain of the estimator relationship and reduces the "stability margin" available to accomodate the modelling error $\left(\mathrm{H}_{0}\right.$ or $\mathrm{B}_{\mathrm{i}}$ in section 3.3 ). A simple numerical example illustrates this.

Let delay $k=3, \sigma_{0}=0.1$ and $\beta=0.99$. From Lemma 1 , 
ROBUSTNESS OF THE PRACTICAL SELF-TUNER

$$
\begin{aligned}
\gamma_{2}{ }^{2} & ={\frac{\beta \sigma}{1-\sigma_{0}}}^{2} \\
\gamma_{3}{ }^{2} & =\gamma_{2}^{2}\left[\beta+\frac{\beta \sigma}{1-\sigma_{0}}\right] \\
& =\left[\frac{\beta \sigma}{1-\sigma_{0}}\right]^{2}
\end{aligned}
$$

Therefore, from Eqn. 12,

$$
\gamma_{e}<1+\gamma_{2}+\gamma_{3}=1.095
$$

Note that when $\sigma_{0}=0$, then $\gamma_{e}=1$.

The results of this subsection provide theoretical confirmation for a well-known fact: the standard matrix gain estimator of section 3.1 works well for non-minimal delays, both in practice and in simulation.

Note that the estimators discussed above are linear in the parameters. In Egardt[33], $1_{\infty}$ stability is shown for the linear full-order controller and for all known integer delays when a different estimator is used (cf Chapter 1). This is a scalar gain estimator which is bilinear in the parameters. It is interesting to note that when Egardt uses an estimator similar to those used here, his proof of $1_{\infty}$ stability is only valid for $k=1$.

\subsection{A ROLE FOR $Q\left(\mathrm{q}^{-1}\right)$}

In the robust stability analysis of Chapters 2 and 3 , it has been implicitly assumed that the error feedback systems considered have solutions in the sense that for each input sequence, there exists a unique and causal output sequence. This property of the feedback systems (existence and uniqueness of a causal solution) will be called well-posedness. It is also known as a realisability condition, (zames[93]). Obviously, the existence of a solution is necessary before 
ROBUSTNESS OF THE PRACTICAL SELF-TUNER

it is possible to discuss its boundedness. More than that however, Zames[93] and many others have shown that well-posedness is an essential property if the error feedback system is to be an accurate representation of physical reality. An example of an ill-posed system will be given below. Further examples are given in Zames[93],[92] and Willems[88]. Note that in considering well-posedness of the feedback system, it is assumed that each operator in the feedback loop is itself an adequate representation of reality $i . e$ well-posed. Throughout this section, ${ }_{0}$ and $B_{i}$ will refer to the feedforward elements in the error feedback systems of section 3.3 , Figs. $3-5$ and $3-6$.

In section 3.4, the design polynomial (or transfer function) $Q\left(q^{-1}\right.$ ) has been assumed to be zero. In the output error case, we obtained:

$$
\mathrm{H}_{\mathrm{O}}=\frac{\mathrm{EA}}{\mathrm{PC}} \mathrm{P}\left(\mathrm{M}_{1}^{-1}-1\right) \frac{1}{\mathrm{P}}
$$

and in the input error case

$$
\mathrm{H}_{i}=\frac{E A B P}{\mathrm{PCA}}\left(\mathrm{M}_{2}^{-1}-1\right) \frac{\mathrm{A}}{\mathrm{PB}}
$$

From Eqns. 16 and 17 , it is obvious that the assumption in Section 3.4 that

Al) causal $M_{1}^{-1}, M_{2}^{-1}$ exist

is needed to ensure the well-posedness of $H_{0}$ and $B_{i}$. If in addition it is assumed that

A2) $M_{i}$ is either linear or if nonlinear, is continuously Frechet differentiable

A.3) $\left(I+\mathrm{H}_{0}\right)^{-1}$ and $\left(I+\mathrm{B}_{i}\right)^{-1}$ exist and are causal $I_{2}$ mappings

then from the results of section 2.5 and recalling that $\mathrm{B}_{\mathrm{cp}}=\left(1+\mathrm{H}_{\mathrm{p}}\right)^{-1}$ (cf Section 3.1), we can represent the exror feedback systems of Figs. 3-7 and $3-8$ as in Fig. 4-2 below: 


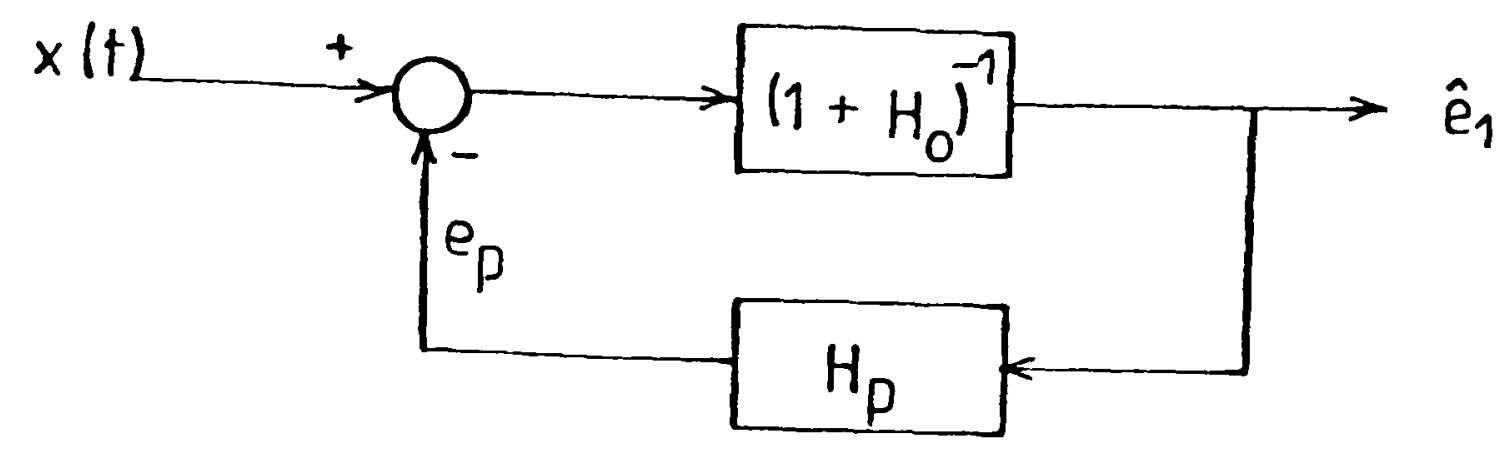

Fig. 4-2 Equivalent Error Feedback system

Lemma I (Well-posedness with $Q=0$ )

If $M_{1}^{-1}, M_{2}^{-1}$ exist and are causal, and if Assumptions $A 2$ and $A 3$ above are valid, then the error feedback system of Fig. 4-2 above is well-posed.

\section{Proof}

Note that $e_{p}(t)=x_{o}^{T}(t-k) \dot{\theta}(t-1)$. The feedback loop of Fig. 4-2 therefore contains a delay of 1 . This ensures that there exists a unique, causal solution for $\hat{e}_{1}(t)$, because the solution for a feedback system can be obtained by iteration and a delay ensures that the iterates converge. The formal proof can be found in zames[93] and willems[88].

ᄆ

Assumption $A 3$ is a standard and much studied condition in input-output stability analysis (eg. Desoer and vidyasagar[28], willems[88]). Comparing with Chapter 2, existence of an inverse for $(1+B)$ is analogous to requiring that the corresponding non-adaptive error feedback systems are well-posed. This appears to be a reasonable requirement if $\mathbf{H}_{0}$ or $\mathbf{B}_{\mathbf{i}}$ exists. Later in this section, some condjtions 
ROBUSTNESS OF THE PRACTICAL SELF-TUNER

for the existence of an inverse operator will be stated.

If $\mathrm{Al}$ is not necessarily true, $\mathrm{H}_{0}$ and $\mathrm{H}_{i}$ may themselves not exist. The reason is apparent from Figs. 3-5 and 3-6, where they are seen to incorporate a feedback loop of the form of Fig. 4-3 below.

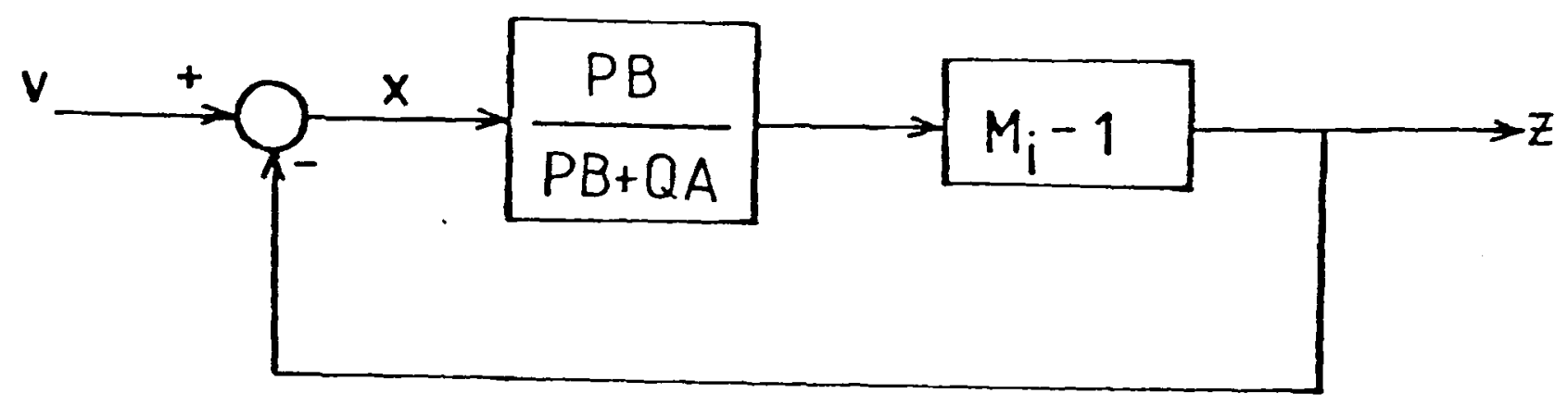

$\begin{array}{ll}\text { Fig. 4-3 } & \text { Possibly ill-posed inner loop of } \\ & \text { Error Feedback System }\end{array}$

In Fig. 4-3, if $Q=0$, the feedback system is well-posed only if a causal $M_{i}^{-1}$ exists. Hence the necessity for Assumption $A$. Two examples illustrate this.

Example I (III-posed feedback loop)

Let $M_{i}$ be the memoryless nonlinearity of Fig. 4-4 (saturation).

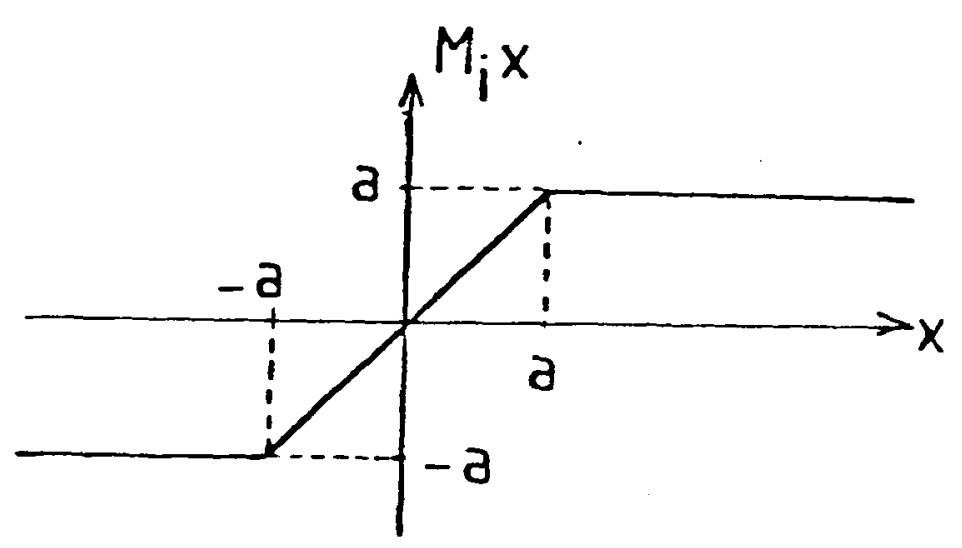

Fig. 4-4 Saturation Nonlinearity 
ROBUSTNESS OF THE PRACTICAL SELF-TUNER

Let $Q\left(q^{-1}\right)=0$. With reference to Fig. 4-3, for every input, v, the output $z$ is given by the point(s) of intersection of the line $z=v-x$ and the graph of $M_{i}-1$. see Fig. 4-5.

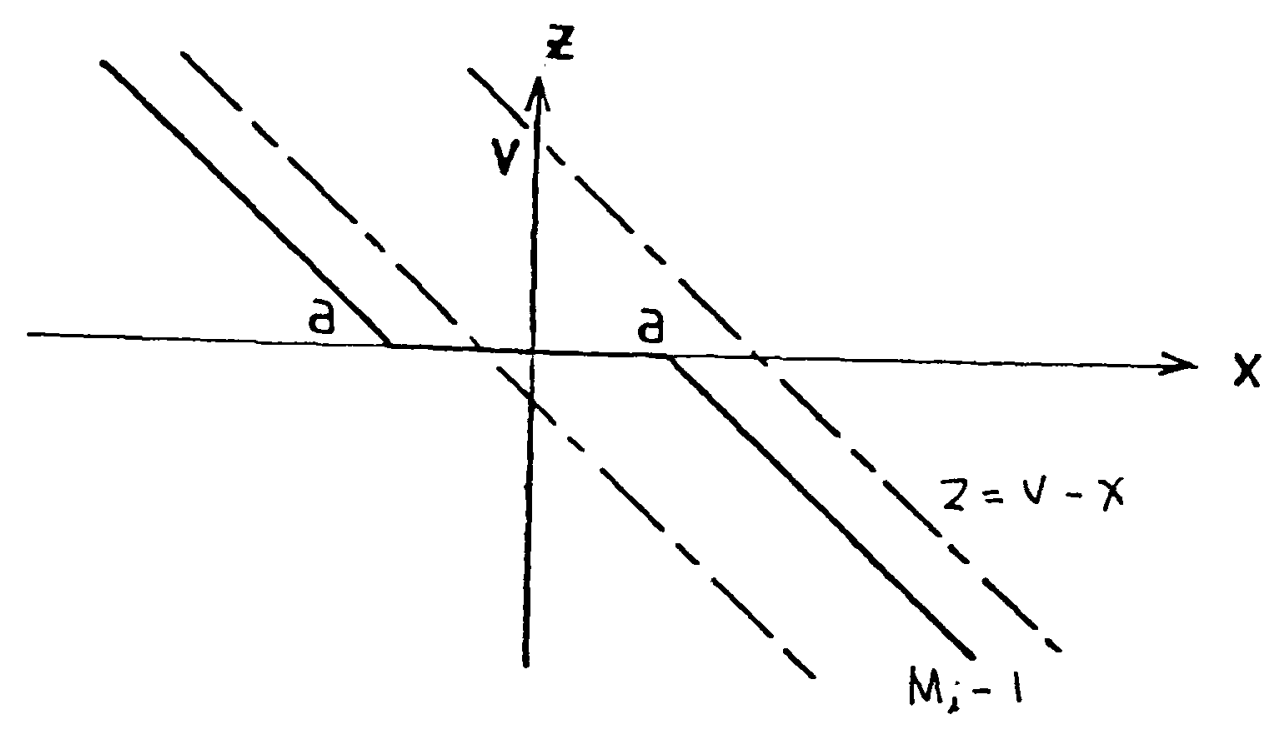

Fig. 4-5 Solutions to Inner Loop of Example 1

From Fig. 4-5, it is clear that there is no solution for all $v$ with $|v|>$ a. Ho thus cannot be well-posed.

Example 2 (Non-causal inverse)

Let $M_{i}=q^{-1}$ (the backward shift operator). This would be the case if the delay in the system is underestimated. When $Q=0$, the feedback loop of Fig. 4-3 reduces to $[q-1]\{q$ being the forward shift operator\}. Hence in this case, a solution exists, but it is not causal.

From the examples above, $Q\left(q^{-1}\right)$ must not be zero if a causal $M_{i}^{-1}$ does not exist. Theorem 1 below states a selection of conditions which will ensure the well-posedness of Fig. 4-3 if Assumption Al is not valid. Denote:

$$
H_{u}=\left[M_{i}-1\right] L\left(q^{-1}\right), \quad \text { where } L\left(q^{-1}\right)=\frac{P B}{P B+Q A}
$$


ROBUSTNESS OF THE PRACTICAL SELF-TUNER

\section{Theorem 1}

When a causal $\mathbf{M}^{-1}$ does not exist, then for the feedback system of Fig. 4-3 to be well-posed, it is sufficient that $Q \neq 0$, and any of the following conditions are met.

(i) $1+\mathrm{H}_{\mathbf{u}}$ is incrementally passive and has finite incremental gain (ii) $M_{i}-1$ has finite incremental gain, $\gamma_{1}$ and $P, Q$ are selected so that $I\left(q^{-1}\right)$ has gain $\gamma_{2}=\sup _{|q|=1}\left|\frac{P B}{P B+Q A}\right|$, such that $\gamma_{1} \gamma_{2}<1$. (iii) $M_{i}-1$ is strictly incrementally conic with centre c and radius $r>0$ and $L\left(q^{-1}\right)$ satisfies either

a) $|c|<x$ and $P, Q$ are selected so that $I\left(e^{j \theta}\right): \quad \theta \in[0,2 \pi]$ lies within the circle (in the complex plane) of

$$
\text { centre } \frac{c}{r^{2}-|c|^{2}} \quad \text { and radius } \quad \frac{r}{r^{2}-|c|^{2}}
$$

OR

$|c|>r$ and $P, Q$ are selected so that $L\left(e^{j \theta}\right): \quad \theta \in[0,2 \pi]$ is outside the circle (in the complex plane) of

$$
\text { centre } \frac{c}{\left.\sqrt{c}\right|^{2}-r^{2}} \text { and radius } \frac{r}{\sqrt[c / 2-r^{2}]{ }}
$$

\section{Proof}

(ii) and ( $i i i)$ are based on the well known contraction mapping theorem, which ensures that if a solution is sought for a feedback system by iteration, the iterates converge to a unique solution. The formal proof can be found in eg. Willems[88] or Desoer and Vidyasagar[28]. The latter also contains the proof for (i) which is less restrictive than either (ii) or (iii) but is more difficult to check. 


\section{Example 1 (Continued)}

With the nonlinearity of Fig. 4-4 above, $\left(M_{i}-1\right)$ is strictly incrementally gain bounded with $\gamma_{1}=1$. Assume that $P=1$ and $Q=\lambda$, a scalar. Let $B\left(q^{-1}\right)=1$ and $A\left(q^{-1}\right)=1-\bar{a} q^{-1}$. In this case, any $\lambda>0$ will ensure that $\gamma_{2}<1$ and hence, Fig. 4-3 is well-posed for any $\bar{a} \in(0,1)$.

\subsubsection{Gain of $\mathrm{H}_{0} / \mathrm{H}_{i}$ with $\mathrm{Q}\left(\mathrm{q}^{-1}\right) \neq 0$}

For simplicity in calculating the gain of $B_{0}$ and $H_{i}$ the robust stability results of section 3.4 were given only for the case of $Q\left(q^{-1}\right)=0$. When considering the linear, higher order case (i.e $M_{i}$ linear $) Q\left(\mathrm{q}^{-1}\right) \neq 0$ does not pose a problem and as section 3.5 indicates

$$
\mathbf{H}=\mathbf{H}_{\mathbf{0}}=\mathbf{B}_{i}=\frac{E\left(A_{0} B-A B_{0}\right)}{\left[\left(P B_{0}+Q A_{0}\right)\right.}
$$

Those robust stability results of section 3.4 , for which $M_{i}$ is linear, are then equally applicable with Eqn. 19 above instead of Eqns. 3-39 or 3-42.

When $M_{i}$ is nonlinear, it is necessary firstly to ensure well-posedness of $\mathrm{H}_{0}$ or $\mathrm{B}_{i}$. As the previous subsection has shown, it may not be permissible that $\mathrm{Q}\left(\mathrm{q}^{-1}\right)=0$. Using the definition of Eqn. 18 and from Figs. 3-5 and 3-6,

$$
\begin{aligned}
& \mathrm{H}_{0}=\frac{A E}{\mathrm{PC}} \mathrm{PB}_{u}\left[1+\mathrm{B}_{u}\right]^{-1} \frac{1}{\mathrm{P}} \\
& \text { where } \mathrm{B}_{u}=\left(\mathrm{M}_{1}-1\right) \frac{\mathrm{PB}}{\mathrm{PB}+Q A} \\
& \mathrm{H}_{i}=\frac{A E}{\mathrm{PC}} \frac{\mathrm{PB}}{\mathrm{A}} \mathrm{B}_{u}\left[1+\mathrm{B}_{u}\right]^{-1} \frac{\mathrm{A}}{\mathrm{PB}} \\
& \text { where } \mathrm{H}_{u}=\left(\mathrm{H}_{2}-1\right) \frac{\mathrm{PB}}{\mathrm{PB}+Q A}
\end{aligned}
$$


ROBUSTNESS OF THE PRACTICAL SELF-TUNER

Note that Theorem $1(i i)$ in section 4.2 essentially requires that for well-posedness of $B_{0^{\prime}}$ the incremental gain of $H_{u}<1$. This is not too restrictive in that for robust stability of the overall closed loop system (Figs. 3-5, 3-6), the gain of $\mathrm{H}_{\mathrm{o}} / \mathrm{H}_{i}$ is necessarily less than $I$ anyway.

There are many possible ways of evaluating the gain of a composition of operators such as $\mathrm{B}_{0}$ or $\mathrm{B}_{i}$ and it is not clear which is best. $A$ simplification occurs when:

a) $M_{i}$ is continuously Frechet differentiable and maps 0 into 0 i.e meets the conditions of Lemma 1 , Section 3.4

and

b) condition (ii) of Theorem 1 above is met (for well-posedness of $\left[1+\mathrm{B}_{\mathrm{u}}\right]^{-1}$, when the incremental gain

$\mathrm{B}_{\mathrm{u}}\left[1+\mathrm{B}_{\mathrm{u}}\right]^{-1} \|_{\Delta}=\gamma_{1} \gamma_{2}\left[1-\gamma_{1} \gamma_{2}\right]^{-1}$.

Lemma 1 of section 3.4 can then be applied to Eqns. 20 or 21 above so that

$$
\operatorname{gain}\left\{\mathrm{B}_{i}\right\}=\operatorname{gain}\left(\mathrm{B}_{0}\right\} \leqslant \sup _{|q|=1}\left|\frac{\mathrm{EA}}{\mathrm{PC}}\right| \gamma_{1} \gamma_{2}\left[1-\gamma_{1} \gamma_{2}\right]^{-1}
$$

Alternatively, if condition (iii) of Theorem 1, section 4.2, is met, then

$$
\left[1+\mathrm{B}_{u}\right]^{-1} \|_{\Delta}=\frac{1+|c| / r}{1-|| 1+c-M_{i} \mid \|_{\Delta}}
$$

where $M_{i}$ is incrementally interior conic with centre and radius $(c, r)$. The gain of $\mathrm{H}_{0}$ or $\mathrm{H}_{i}$ follows by composition of gains. 
ROBUSTNESS OF THE PRACTICAL SELF-TUNER

\subsection{OFFSETS}

In the discussion of the basic clarke-Gawthrop self-tuner in Chapters 2 and 3 , the problem of offsets, defined here as the steady state(constant) difference between the auxiliary output, $\Phi(t)$ and the filtered setpoint, $R\left(q^{-1}\right) w(t-k)$, was largely ignored. offsets can arise from two situations:

a) when the filtered setpoint, $R\left(q^{-1}\right) w(t-k)$ has a non-zero mean and $Q\left(q^{-1}\right) \neq 0$

b) when the disturbances have a non-zero mean, $\bar{d} \neq 0$ in Fig. $2-2$ i.e. the nominal plant is now

$$
A\left(q^{-1}\right) Y(t)=B\left(q^{-1}\right) u(t-k)+C d(t)+\bar{d}
$$

In situation (a), consider the non-adaptive clarke-Gawthrop self-tuner controlling the nominal plant (cf Chapter 2). The closed loop system is ( if the parameters of $A\left(q^{-1}\right), B\left(q^{-1}, C\left(q^{-1}\right.\right.$ ) are exactly known)

$$
y(t)=\frac{B R}{P B+Q A} w(t-k)+\frac{E B+Q C}{P B+Q A} d(t)
$$

If $Q=0$, then the steady state offset is zero if $d(t)$ has a zero mean. If $Q \neq 0$, then to get zero steady offset, $Q(1)=0$. The possibilities for $Q$ are:

$$
\begin{array}{ll}
Q\left(q^{-1}\right)=\lambda\left(1-q^{-1}\right) & \text { OR } \\
Q\left(q^{-1}\right)=\lambda\left(1-q^{-1}\right) /\left(1-\alpha q^{-1}\right) & (\text { see clarke and Gawthrop }[20])
\end{array}
$$

When the disturbances have a non-zero mean, $\bar{d}, Q(1)=0$ is obviously not sufficient to ensure zero steady state offset. In general the methods available to deal with this problem fall into two categories. The first consists of methods which estimate the mean of the disturbances, $\bar{d}$. The second category consists of methods which somehow 
ROBUSTNESS OF THE PRACTICAL SELF-TUNER

include an integrator in the control law. The main aim of this section is to extend the robust stability results for the basic self-tuner (cf Chapter 3) to include self-tuners which have been modified to cope with these offsets. Only the clarke-Gawthrop self-tuner will be considered.

\subsubsection{Estimating Mean Level of Disturbance, $\bar{d}$}

In this category, the estimator is modified to explicitly estimate $\bar{d}$. The simplest method is to augment the data vector, $x(t)$.

\section{Augmented Data Vector}

$$
\begin{array}{rlrl}
\text { Let } x_{0}^{T} & \cong \frac{1}{c}\left[u_{0}(t), u_{0}(t-1), \ldots ; y_{0}(t), y_{0}(t-1), \ldots ; 1\right]: \text { cf Eqn. } 2-20 \\
\theta(t) & \cong\left[g_{0}, g_{1}, \ldots ; f_{0}, f_{1} \ldots ; ; \bar{d}\right] & : c f \text { Eqn. } 2-17
\end{array}
$$

In this method, the constant disturbance, $\bar{d}$, is regarded as just another parameter to be estimated, using the same estimator as that discussed in section 3.1. Not surprisingly, the error feedback systems are unchanged and only the inputs to the exror feedback system are modified. Instead of Eqn. 2-33, we get as a relationship between the exror quantities

$$
\hat{e}_{1}(t)=e(t)-e_{m}(t)-e_{p}(t)+\left(\frac{E}{c}-1\right) \bar{d}
$$

Comparing with Eqns. 2-35, 2-37, $d(t)$ is replaced by

$$
d_{1}(t)=d(t)+\frac{1}{c} \bar{d}
$$

The robust stability analysis of the clarke-Gawthrop self-tuner, presented in Chapters 2 and 3 , is thus valid with the above modifications to the inputs of the error feedback systems, when the data and parameters vectors are augmented as described above. Note however that with a 
ROBUSTNESS OF THE PRACTICAL SELF-TUNER

constant $\bar{d}$ at the input to the error feedback system, it is the $l_{\infty}$ results which will be more useful.

The disadvantage of this method is that the estimated dc level can only change at the same rate as the other parameter estimators (i.e. it has the same forgetting factor). It is sometimes desirable to have a different forgetting factor for the estimated dc level, eg. when it is time-varying. A popular solution then is to estimate the mean level, $\overline{\mathbf{d}}$ separately. (See eg. Clarke[21])

\section{Separate dc estimation}

Let $\hat{\mathrm{d}}(t) \equiv$ estimate of $\overline{\mathrm{d}}$

$\beta_{\mathrm{dc}} \cong$ forgetting factor for $\hat{\mathrm{d}}$ estimation.

With the same data and parameter vectors as for the standard matrix gain estimator, [described by Eqns. 3-1 to 3-3], the combined estimator (i.e. including $\hat{d}$ estimation) can be described as

$$
\begin{aligned}
& \hat{e}_{1}(t)=\Phi(t)-x_{0}^{T}(t-k) \hat{\theta}(t-1) \\
& \hat{\theta}(t)=\hat{\theta}(t-1)+s^{-1}(t-k) x_{0}(t-k)\left[\hat{e}_{1}(t)-\hat{d}(t-1)\right] \\
& \hat{d}(t)=\hat{d}(t-1)+\left(1-\beta_{d c}\right)\left[\hat{e}_{1}(t)-\hat{a}_{1}(t-1)\right] \\
& s(t)=\beta S(t-1)+x_{0}(t) x_{0}^{T}(t) \\
& s(0)=s_{0}>0 \\
& \text { Define: } e_{1}(t) \equiv \hat{e}_{1}(t)-\hat{d}(t-1)
\end{aligned}
$$

To derive an error feedback system for the clarke-Gawthrop self-tuning controller when this estimator is used, we need a relationship between the error quantities which corresponds to Eqn. 2-33. With the nominal system of Eqn. 23 , we get

$$
\hat{e}_{1}(t)+e_{p}(t)-\bar{d}=e(t)-e_{m}(t)+\left(\frac{E}{c}-1\right) \bar{d}
$$


ROBUSTNESS OF THE PRACTICAL SELF-TUNER

where $e_{,} e_{m^{\prime}} \hat{e}_{1}, e_{p}$ are identical to those in section 2.3.2.

Corresponding to Lemma 1 of Section 3.1, we have

\section{Lerma 1}

Let $H_{c p}^{d}$ denote the functional relationship between $e_{1}(t)$ and $\left[e_{1}(t)+e_{p}(t)\right]$. The gain of $B_{c p}^{d}$ is $\left(1-\sigma_{0}^{d}\right)^{-1 / 2}$ i.e.

$$
\sum e_{1}^{2}(t) \leqslant\left(1-\sigma_{0}^{d}\right)^{-1} \sum^{N}\left[\hat{e}_{1}+e_{p}(t)-\bar{d}\right]^{2}: \forall N>t_{0}
$$

where $\sigma_{0}^{d}=1-\beta_{d c}+\sigma_{0}$

and $\sigma_{0}=\sup _{t>t_{0}} x^{T}(t) s^{-1}(t) x(t)$ :as in section 3.1

Proof In Appendix C.3

$\mathrm{H}_{\mathrm{CP}}^{\mathrm{d}}$ represents a 'combined' estimator. With a $\hat{\mathrm{d}}$ estimator, the control law, (cf Eqn. 2-26) is

$$
u_{0}(t)=\frac{1}{Q}\left[R w(t)-\hat{\Phi}_{k}(t+k / t)-\hat{d}(t)\right]
$$

In the output error case, with delay $k=1$, the error feedback system corresponding to Fig. $3-5$ is (with this estimator) in Fis. 4-6

As far as robust stability is concerned, the main difference from Section 3.4 is that the estimator relationship is $\mathrm{H}_{\mathrm{CP}}^{\mathrm{d}}$ instead of $\mathrm{H}_{\mathrm{CP}}$. Lemma $I$ shows that $\mathrm{H}_{\mathrm{Cp}}^{\mathrm{d}}$ will always have a larger gain than ${ }_{\mathrm{Cp}^{\prime}}$ whenever $\beta_{d c}<1$. This implies that the use of a separate $\hat{d}$ estimator is 'destabilising' in the sense that the gain of $\mathrm{H}_{0}$ ( or $\mathrm{H}_{i}$ ) must be smaller than in the case when $\bar{d}$ is estimated by the augmented data method previously discussed. A similar conclusion can be drawn for the input error case. The stability analysis of Fig. 4-6 is similar to those in 


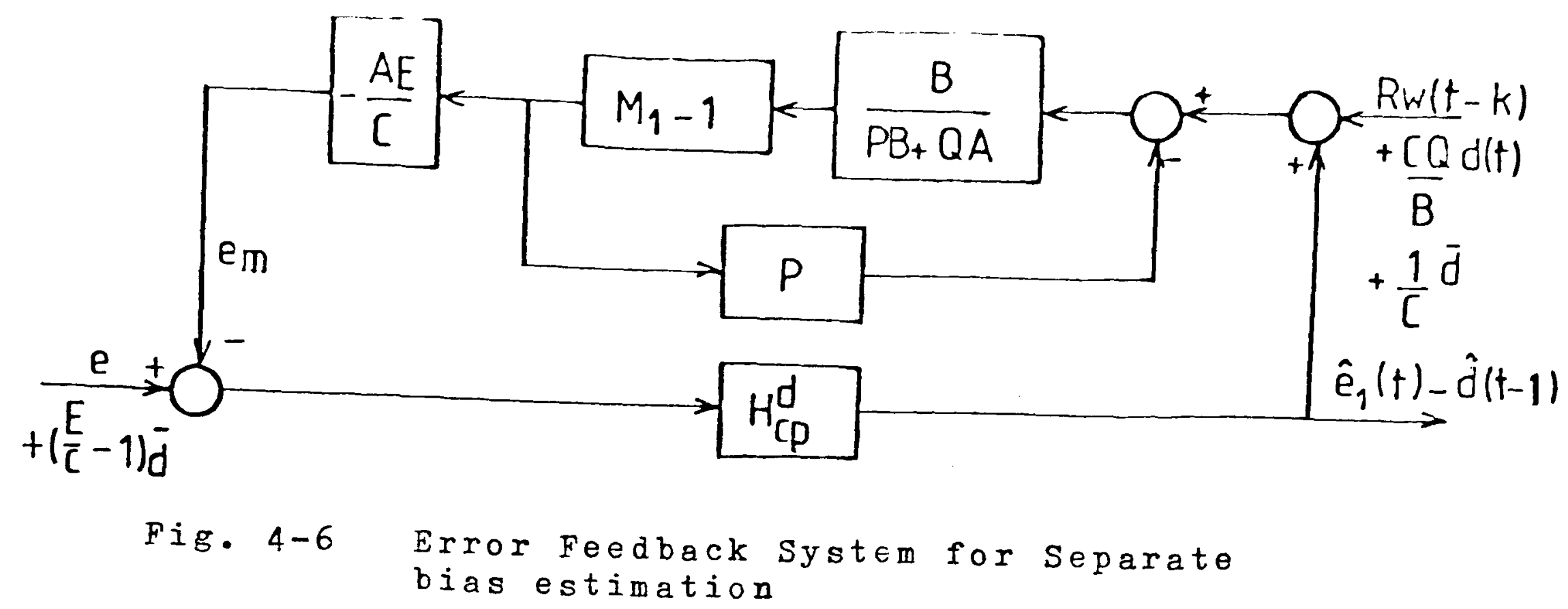

Chapter 3, with appropriate modification to the error inputs, and will not be repeated here.

If the control law of Eqn. 34 is used, with either of the two $\hat{d}$ estimation schemes discussed, the offset will be eliminated if $\hat{a}(t) \rightarrow \bar{d}$. As this cannot be guaranteed, particularly in the presence of nonlinearities or higher order dynamics, the next category of methods for offset elimination seeks to include an integrator in the control law. (see eg. Moden[67])

\subsubsection{Integrator Methods}

The methods to be discussed in this subsection have all been studied elsewhere with respect to their efficiency in removing offsets. The main purpose here is to examine the robust stability of the clarke-Gawthrop self-tuner used in combination with these methods. These are

a) estimating an incremental predictor model, Peterka[74]

b) using an inner loop compensator, clarke and Gawthrop[20] 
ROBUSTNESS OF THE PRACTICAL SELF-TUNER

c) using an outer loop compensator, clarke and Gawthrop[20]

Methods (b) and (c) have also been discussed in detail by Moden[67], who shows that they are equivalent (in the linear full order case) to specific choices of the design polynomial, $P\left(q^{-1}\right)$.

\section{a) Incremental Predictor}

Assume that the mean level of the disturbances $\bar{d}$, is constant. For the nominal system of Eqn. 23, an incremental model can be written as

$$
A\left(q^{-1}\right) Y^{\prime}(t)=B\left(q^{-1}\right) u^{\prime}(t-k)+C\left(q^{-1}\right) d^{\prime}(t)
$$

where' denotes data differencing eg. $y^{\prime}(t)=y(t)-y(t-1)$.

A predictor can be written for the system of Eqn. 35 as

$$
\Phi \cdot{ }^{\star}(t+k / t)=\frac{F}{C} Y \cdot(t)+\frac{G}{C} u \cdot(t)
$$

For simplicity, consider the minimal delay case, $k=1$. At time $t$, the predicted value of $\Phi(t+k)$ is given by

$$
\begin{aligned}
\hat{\Phi}(t+k / t) & =\hat{\Phi} \cdot(t+k / t)+\Phi(t) \\
& =\Phi(t)+x \cdot T(t) \hat{\theta}(t)
\end{aligned}
$$

When such an incremental model is assumed for the nominal system, it is a simple but tedious matter to show that the error feedback system for the output error case is as shown in Fig. 4-7 below.

The functional relationship representing the incremental estimator, $H_{c p}$ ' has by analogy to section 3.1 , a gain of $\left(1-0_{0}\right)^{-1 / 2}$ where

$$
\sigma_{0} \cdot=\sup _{t>t} x_{0} \cdot T(t-k) s^{\cdot-1}(t-k) x_{0} \cdot(t-k)
$$




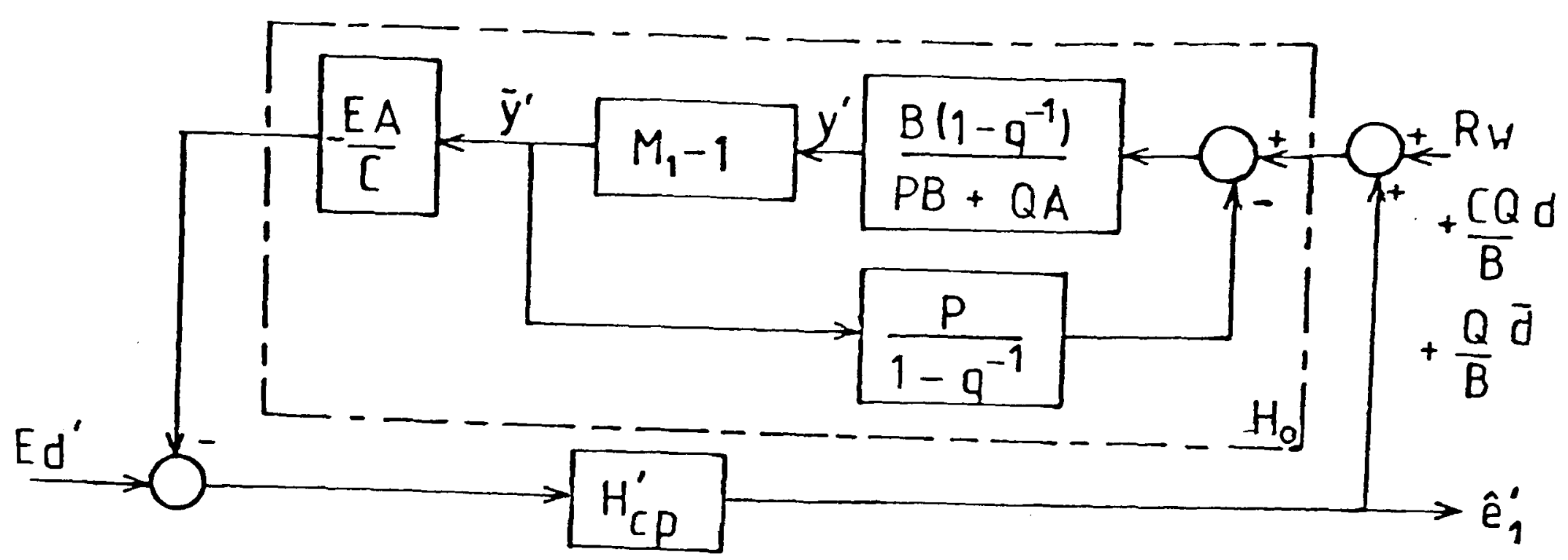

$\begin{array}{ll}\text { Fig. } 4-7 & \text { Error Feedback System for Incremental } \\ & \text { Predictor }\end{array}$

Fig. 4-7 for the incremental predictor corresponds to Fig. 3-5 for the ordinary predictor. As for the ordinary estimator, it is difficult to put a priori bounds on $\sigma_{0} \cdot$. The conditions for small $\sigma_{0}$, discussed in section 3.7, are also valid for $\sigma_{0}^{\prime}$ if applied to the differenced data vector, $X^{\prime}$. Simulation studies suggest that it is usually small. Aside from ${ }_{\mathrm{CP}} \cdot$, the other difference due to an incremental predictor is in the differencing factor, $\left[1-q^{-1}\right]$.

Let $Q=0 . \mathrm{H}_{0}$ ' of $\mathrm{Fig}$. 4-7 reduces to

$\mathrm{H}_{0} \cdot=\frac{E A}{\mathrm{C}}\left[\mathrm{M}_{1}{ }^{-1}-1\right]{\frac{1-\mathrm{q}^{-1}}{\mathrm{P}}}^{-1}$

The closed loop output, (cf Eqn. 3-47) is

$y_{0}(t)=\frac{1}{p}\left[\hat{e}_{1} \cdot(t)+R w(t-1)\right]$

Hence if the incremental prediction error $\hat{e}_{1} \cdot(t)$ is bounded, then so is $y_{0}(t)$ (assuming bounded setpoint), even though there is a constant disturbance $\overline{\mathrm{d}}$. 
ROBUSTNESS OF THE PRACTICAL SELF-TUNER

While this method is sound in principle, there are practical difficulties involved in data differencing. If for instance the outputs or inputs are constant over long periods of time, there will be numerical problems in the estimator as the differenced data vector is filled with o's.

A very similar method is to remove mean levels from the input-output data before estimation, by high pass filtering: clarke[21]. The analysis is similar to that above.

The next two methods explicitly include an integrator in the feedback loop.

b) Cascaded (Inner Loop) Compensator

In this method, the self-tuner is placed in sexies with a compensator, $\mathrm{L}_{\mathrm{c}}\left(\mathrm{q}^{-1}\right)$, as shown in Fig. 4-8 below.

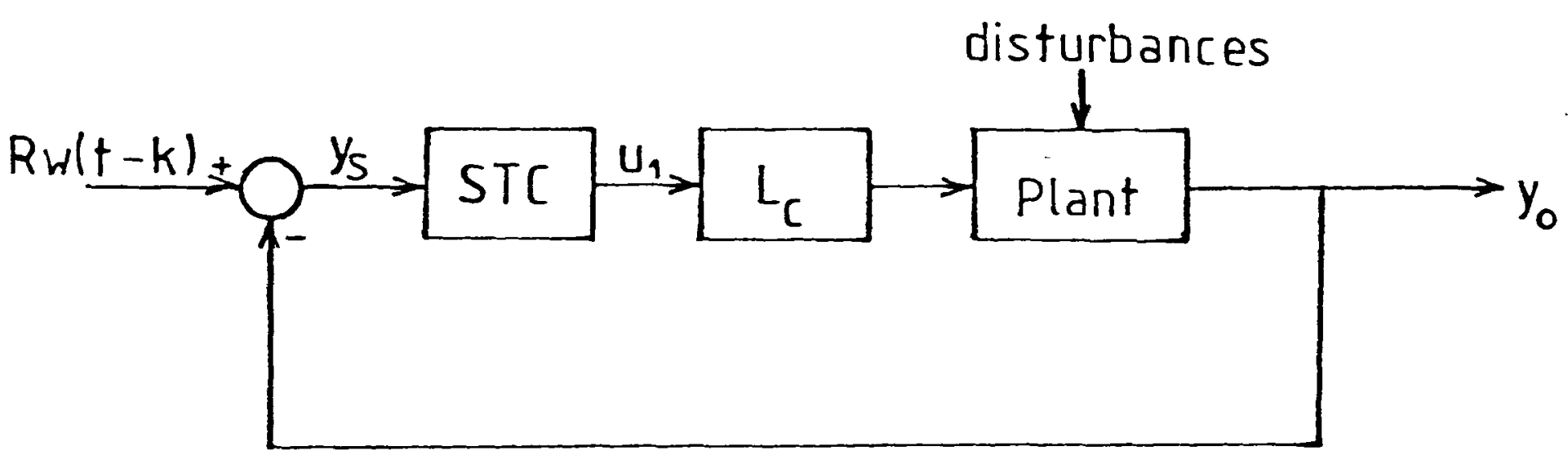

Fig. 4-8 Self-Tuning Control with Cascaded
Compensation

The self-tuner sees an expanded plant, which includes the compensator. A prediction model is obtained for the error signal, $P_{Y_{s}}(t)=P_{0}(t)-R w(t-k)$. The control law sets the $k$ steps ahead prediction of $\mathrm{Py}_{\mathbf{g}}(t)$ to zero. The simplest compensator to remove offsets 
ROBUSTNESS OF THE PRACTICAL SELF-TUNER

would be a pure integrator. If this is not desirable, a proportional and integral type of compensator can be used. Here, we consider the latter i.e.

$L_{c}\left(q^{-1}\right)=K\left(1-\alpha q^{-1}\right) /\left(1-q^{-1}\right): \alpha<1, K$ is the compensator gain (41) For the linear full order case, a prediction model is

$$
\left\{P y_{g}(t+k / t)\right\}^{\star}=F\left(1-q^{-1}\right) y(t)+\operatorname{EBK}\left(1-\alpha q^{-1}\right) u_{1}(t)
$$

where the E, $F$ polynomials are the same as those for the non-adaptive clarke-Gawthrop self-tuner discussed in Chapter 2. Note that in the adaptive case, the data and parameter vectors must be expanded to accomodate the compensator poles and zeros.

Moden[67] has shown that the same control law can be obtained from the Clarke-Gawthrop self-tunex by using a special reference model, $\frac{1}{p}, a$ scalar $Q$ and a setpoint filter, $R=\mathbf{P}$.

It can easily be shown that in the linear full order case, stability depends on the roots of $P B+Q^{\prime} A$ where $Q^{\prime}=\frac{Q}{L_{C}}$. If $L_{C}$ is chosen as in Eqn. 41, the stability polynomial is

$$
\operatorname{PBK}\left(1-\alpha q^{-1}\right)+Q\left(1-q^{-1}\right) A
$$

This will be used for a comparison later.

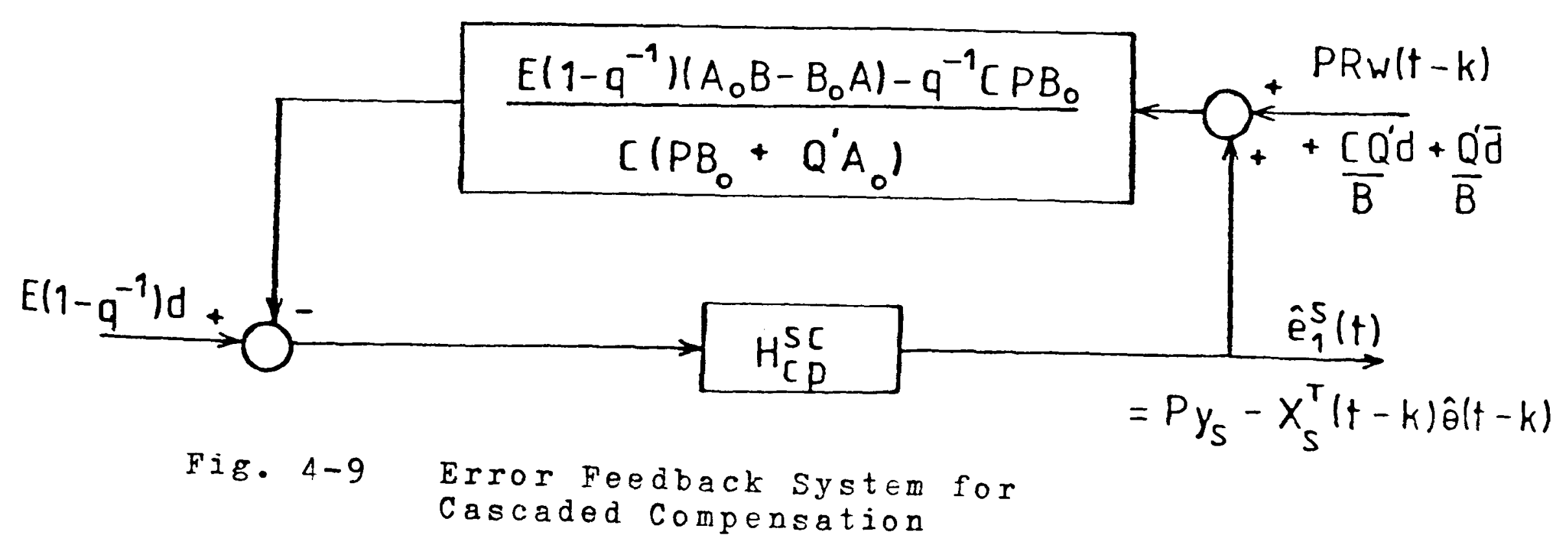


ROBUSTNESS OF THE PRACTICAL SELF-TUNER

The robust stability analysis of this form of offset elimination is rather complicated. Here, only the error feedback system for the linear, higher order case will be presented in Fig. 4-9 above. Robust stability of this feedback system can be analysed with methods similar to those discussed in Chapter 3. Note that $x_{s}$ is a data vector which contains the $Y_{s}$ and $u_{1}$ of Fig. $4-8$ and $B_{c p}^{s C}$ is an estimator relationship using the same algorithm as section 3.1 but with $x_{g}$ as the data vector.

\section{c) Outer Loop Compensation}

The third method for including an integrator is shown in Fig. 4-10 below.

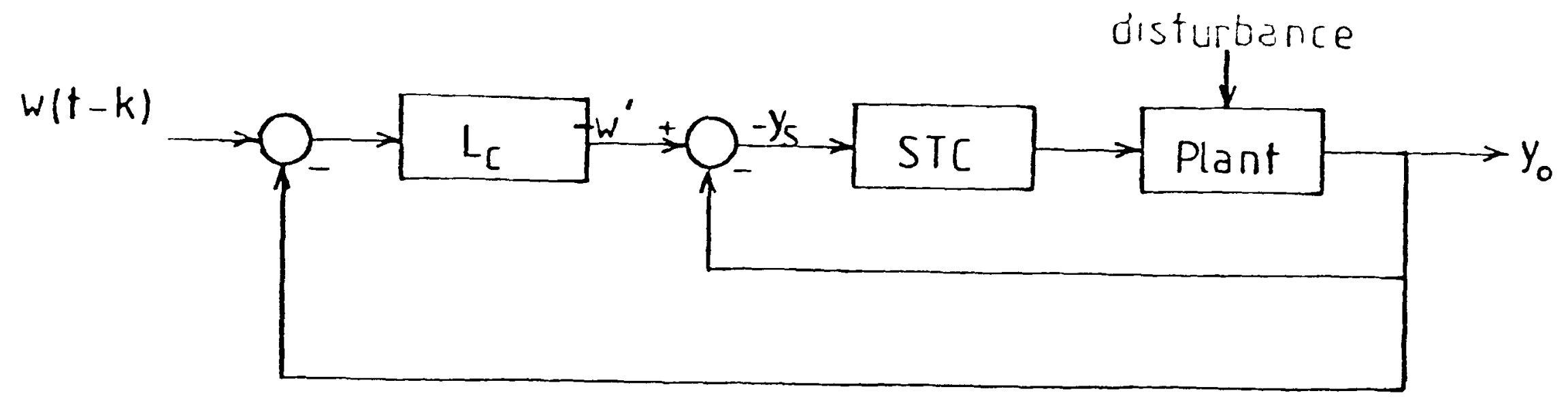

Fig. 4-10 Self-Tuning Control with outer Loop
Compensation

An outer loop is added and the self-tuner estimates a prediction for $\Phi_{s}(t)$, in terms of past values of $y_{s^{\prime}} w^{\prime}(t)$ and $u(t)$

where $\quad y_{\mathbf{g}}(t)=y_{0}(t)-w^{\prime}(t-k)$

and $w^{\prime}(t)=I_{c}\left(q^{-1}\right)\left[y_{0}(t)-w(t)\right]$

For the non-adaptive linear full order case, a predictor for $y_{\mathbf{g}}$ can be formulated as

$$
\begin{aligned}
\Phi_{S}{ }^{*}(t+k / t) & =P Y_{S}{ }^{*}(t+k / t) \\
& =\frac{F}{C} Y_{s}(t)+\frac{G}{C} u(t)-\frac{E A}{C} W^{\prime}(t)
\end{aligned}
$$


ROBUSTNESS OF THE PRACTICAL SELF-TUNER

Where $F$ and $G$ are the usual controller parameters as derived in Chapter 2.

The control law is then

$\hat{\Phi}_{s}(t+k / t)+Q u(t)=0$

With this control law and with the PI type of outer loop compensator, $I_{c}($ Eqn. 4l), the closed loop characteristic polynomial in the linear full order case is

$\ddot{P B}+Q A\left(1-q^{-1}\right)$

where $\dot{P}\left(q^{-1}\right)=P\left(q^{-1}\right)\left[1-q^{-1}-q^{-k} k\left(1-\alpha q q^{-1}\right)\right]$

Consider the simple case of $Q=0, k=1$. From Eqns. 48 and 49 the characteristic polynomial is

$$
\dot{P B}=\mathrm{PB}\left[1-(\mathrm{K}+1) \mathrm{q}^{-1}+\mathrm{K} \alpha \mathrm{q}^{-2}\right]
$$

Eqn. 50 shows that the choice of the gain, $\mathrm{K}$ and compensator zero, $\alpha$ for the outer loop compensator is critical for stability. If a pure integrator is used, $\alpha=0$ and any positive $\mathrm{K}$ will give an unstable pole to the closed loop system. Even if $\alpha>0$, there is minimum $K$, beneath which Eqn. 50 will have an unstable root. Bence 'tuning' a PI compensator for the outer loop method is more difficult than it is for the inner loop method (cf Eqn. 43). One possible solution is to always include some control weighting, $Q \neq 0$.

When considering robust stability, the error feedback system, corresponding to Fig. 3-5 is shown in Fig. 4-11. (for the output error case and $k=1$ ) 


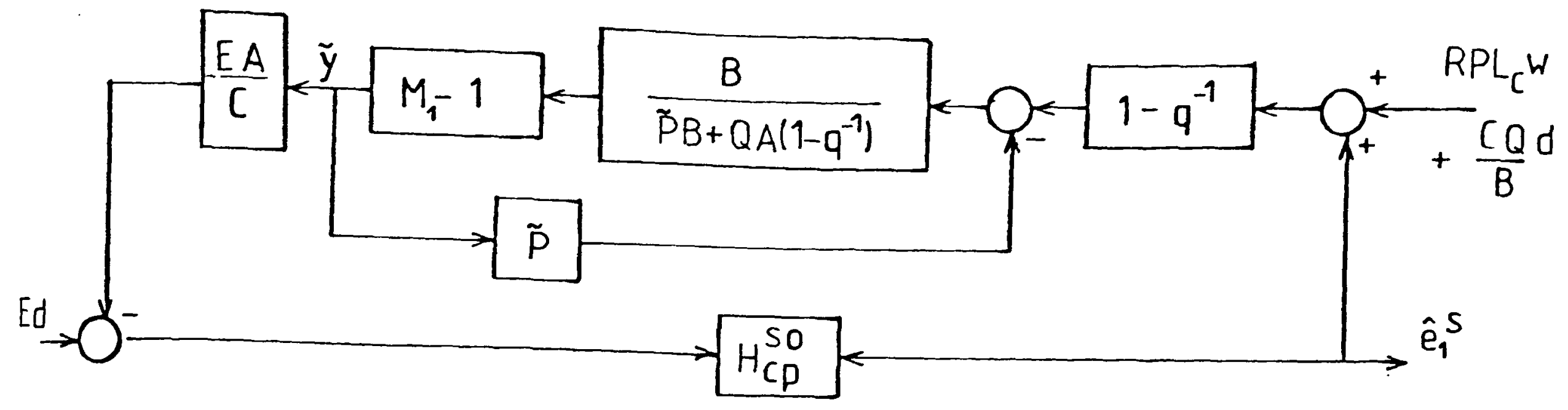

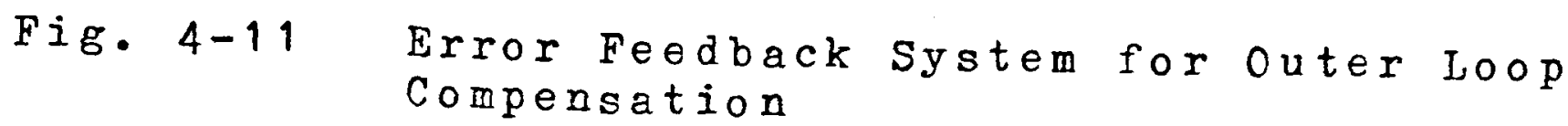

$\mathrm{B}_{\mathrm{Cp}}^{\mathrm{so}}$ represents an estimator similar to $\mathrm{H}_{\mathrm{Cp}}$ of section 3.1, except that the data vector, $x_{s}^{0}$ now contains $y_{s}(t), u_{0}(t)$ and $w^{\prime}(t)$. The gain of $\mathrm{H}_{\mathrm{cp}}^{\mathrm{so}}$ is thus also $\left(1-\sigma_{0}^{\mathrm{so}}\right)^{-1 / 2}$ where

$\sigma_{0}^{s 0}=\sup _{t>t_{0}} x_{s}^{o T}(t) s^{-1}(t) x_{s}^{0}(t)$

Note that for convenience, $\bar{d}$ has been incorporated in $d i . e . d(t)$ has non-zero mean.

since $w^{\prime}(t)$ is a filtered error, it is more likely to be 'persistently exciting' even when the setpoint $w(t)$ is constant, and bearing in mind the results of section 3.7 , it is a reasonable postulate that $\sigma_{0}^{\text {so }}$ is small.

\section{Some Remarks}

It is difficult to make a direct comparison of the three methods discussed above, partly because the estimators in each case, ${ }_{C p}{ }^{\prime},{ }_{C p}{ }^{s C}$ $\mathbf{B}_{\mathrm{cp}}^{\text {so }}$ have different data fed into them and hence it is impossible to say a priori what their gains are. 
ROBUSTNESS OF THE PRACTICAL SELF-TUNER

From the stability point of view, the compensator parameters for the outer loop method need to be more carefully tuned than for the inner loop method. However as Moden[67] points out, the outer loop method is easier to implement from an algorithmic point of view.

For an example, assume that $Q=0$ and that $M_{1}$ is Iinear. Assume also that in all three 'integrating' methods considered, the estimators have a small gain. Table 1 below shows the corresponding $H_{0} \cdot s$ [from Figs. 4-6, 4-9, 4-11]

\section{Table 1}

$\mathbf{B}_{\mathbf{0}}$

Incremental Predictor

$\frac{E A}{P C}\left(1-q^{-1}\right)\left(M_{1}^{-1}-1\right)$

Cascaded PI

$\frac{E A}{P C}\left(1-q^{-1}\right)\left(M_{1}^{-1}-1\right)-q^{-1}$

Outer Loop PI

$$
\tilde{p}-1 \frac{E A}{C}\left(1-q^{-1}\right)\left(M_{1}^{-1}-1\right)
$$

In each case, the Nyquist locus of $\mathrm{H}_{0}$ must lie within some critical circle (depending on estimator gain). Other than a differencing factor, the incremental predictor case is similar to the ordinary controller of Chapter 3. The requirement for the cascaded PI case may be more difficult to meet due to the extra additive delay. As in the linear full order case, the parameters of the offset compensator influence stability for the outer loop method (in P) but not for the inner loop method.

A fourth method for including integral action, which has been proposed in Clarke and Gawthrop[20] and discussed in Moden[67], is to include integrators in both the reference model, $\mathbf{P}$ and the setpoint 
USTNESS OF THE PRACTICAL SELF-TUNER

ters, so that $P(I)=R(I)=\infty$. This method fits easily into the lysis of Chapter 3 and will not be further discussed here. 
CHAPTFR 5

DF.SIGN FXAMET.FS

\subsection{INTRODUCTION}

In this chapter, some examples of self-tuning applied to systems with nonlinearities or which are of higher order than the controller are simulated.

It is shown that different design choices, while giving the same robust stability margins, can result in very different control performance with respect to other criteria.

In the case of nonlinear systems, it appears reasonable to treat the results of the previous chapters as design guidelines, even though it may not be possible to satisfy the robust stability conditions rigorously.

\section{I DFSIGN CHOICES}

In Chapters 3 and 4 , conditions have been specified for the robust stability of a class of systems under self-tuning adaptive control and in Chapter 2, for robust stability and disturbance attenuation performance of controllers designed with the nonadaptive design method. To recapitulate, the design choices available to the engineer are 
1. The assumed system order (i.e. number of $g$ and $f$ parameters in the parameter vector).

2. The estimator: in this thesis, the choice considered is between a scalar gain or a matrix gain recursive estimator.

3. The design polynomials: $P\left(q^{-1}\right), Q\left(q^{-1}\right), R\left(q^{-1}\right)$ and $c\left(q^{-1}\right)$

4. The various methods for rejecting unneasurable joad disturbances and/or tracking non-zero setpojnts which were discussed in Chapter 4.

In adaptive control, the upper limit on the assumed system order is often determined in practice by computational requirements. In most reported applications, a first or second order model seems adequate for process control.

Analysis for both matrix gain and scalar gain type estimators has been presented in chapter 3. In the self-tuning control literature, matrix gain estimators seem to be preferred in applications and it is an empirical fact that they are 'faster' than scalar gain estimators. Historically however, some of the first stability analysis available were for scalar gain estimators eg. Fgardt[33] and Goodwin et al[43]. In this chapter, matrix gain estimators will be used except where otherwise stated.

In the next two sections, simulation examples will be presented to illustrate the performance of some systems under adaptive self-tuning control, for the different design choices above. These will serve partly to clarify some of the less obvious results of the previous chapters. 
The primary motivation, however, is that robust stability is just one control design criterion and it ig interesting to see how a design towards this criterion will measure up to other criteria such as tracking, disturbance rejection and speed of response.

\subsection{TITNEAR SYSTFMS}

In this section, examples of systems with linear perturbations will be considered. Using the notation of the previous chapters, this implies that

$M_{1}$ or $M_{2}=\frac{A B}{R A} 0=$ linear plant perturbation

Recall that in the absence of plant perturbations, the design polynomials have specific interpretations (cf Chapter 1 ). $\frac{I}{p}$ is the reference model, $\frac{l}{Q}$ is the compensator on predicted tracking error, $R$ i.s the setpoint filter and $c$ contains the predictor or observer poles.

\section{Fxample I}

Consider the second order system in Fxample 3 of section 3.4.3. The plant is described by

$$
\begin{aligned}
& A_{0}=1-1.6 q^{-1}+0.8 q^{-2} \\
& B_{D}=1.6+0.2 q^{-1} \\
& k=\text { integer delay }=1 \\
& d(t)=\text { zero mean output noise with an amplitude variance of } 0.2
\end{aligned}
$$

This system is simulated over a time period of 2000 samples, of which only the last 1000 will be displayed. Figs. 5-ra,b show the output and input of this system under the control of a self-tuner with the following specifications: 


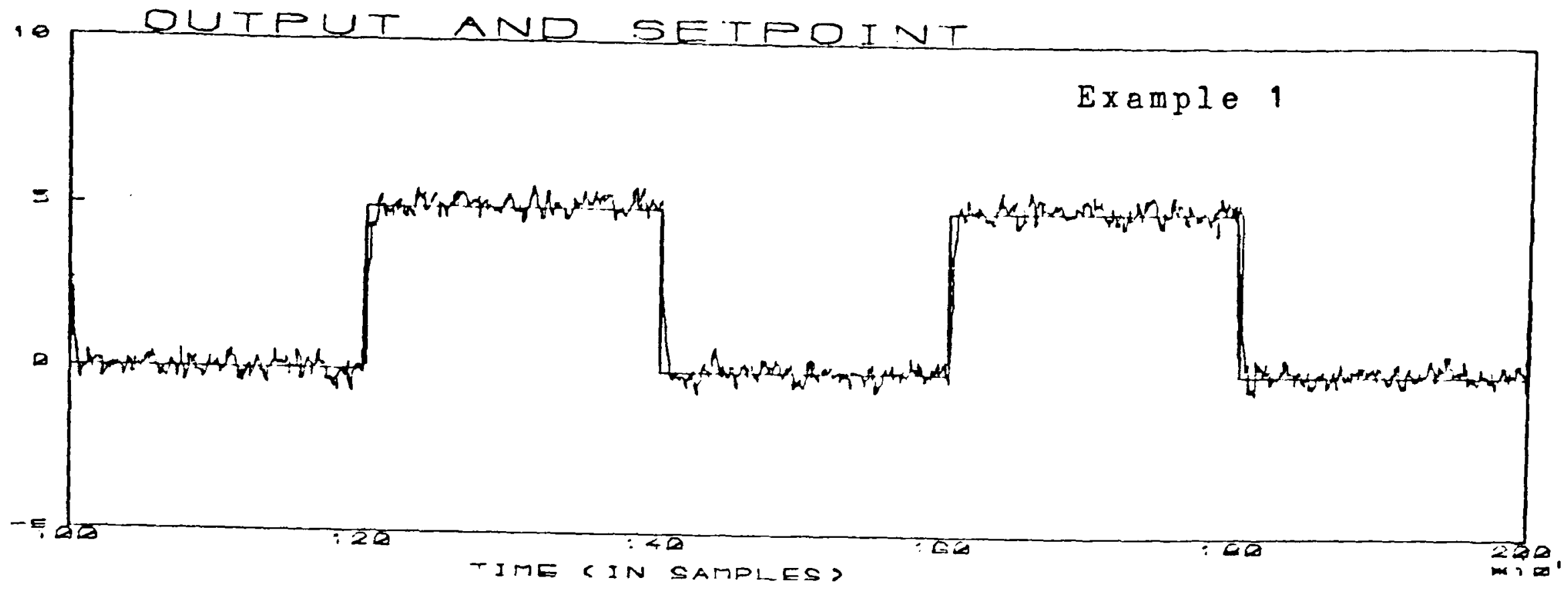

Fig. 5-1a Full order Controller with lst order P

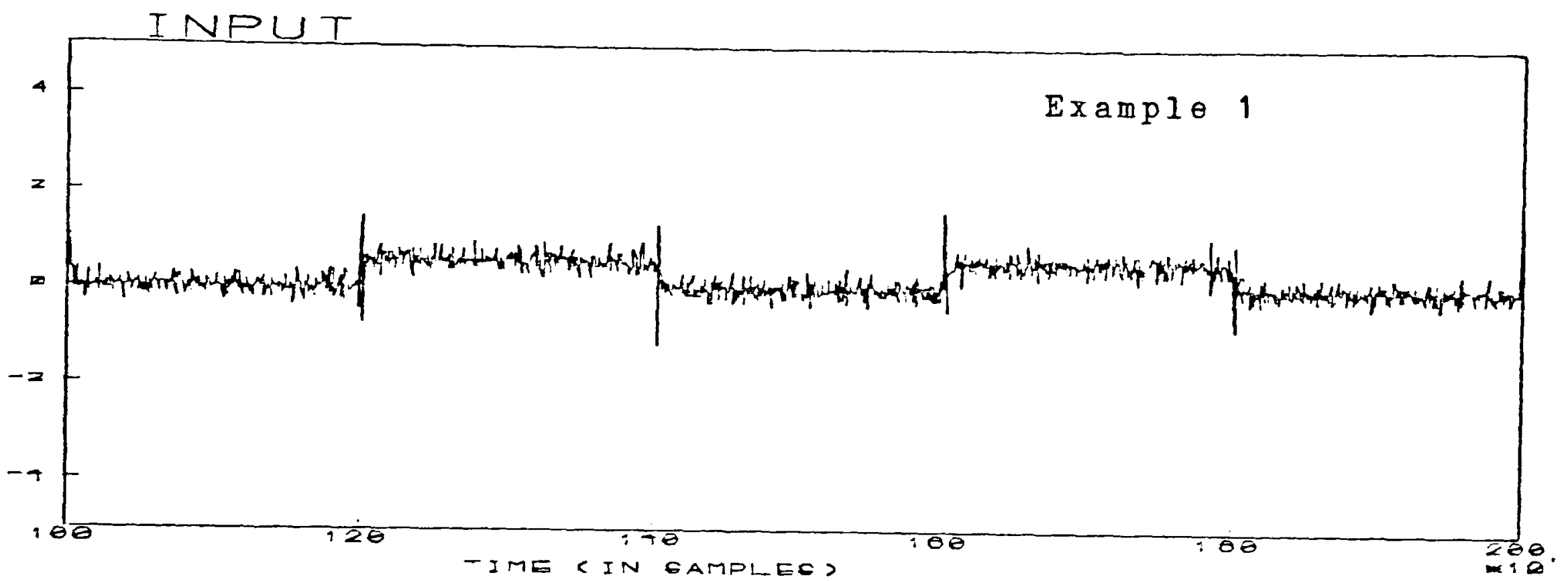

Fig. 5-1b Full order Controller with lst order P

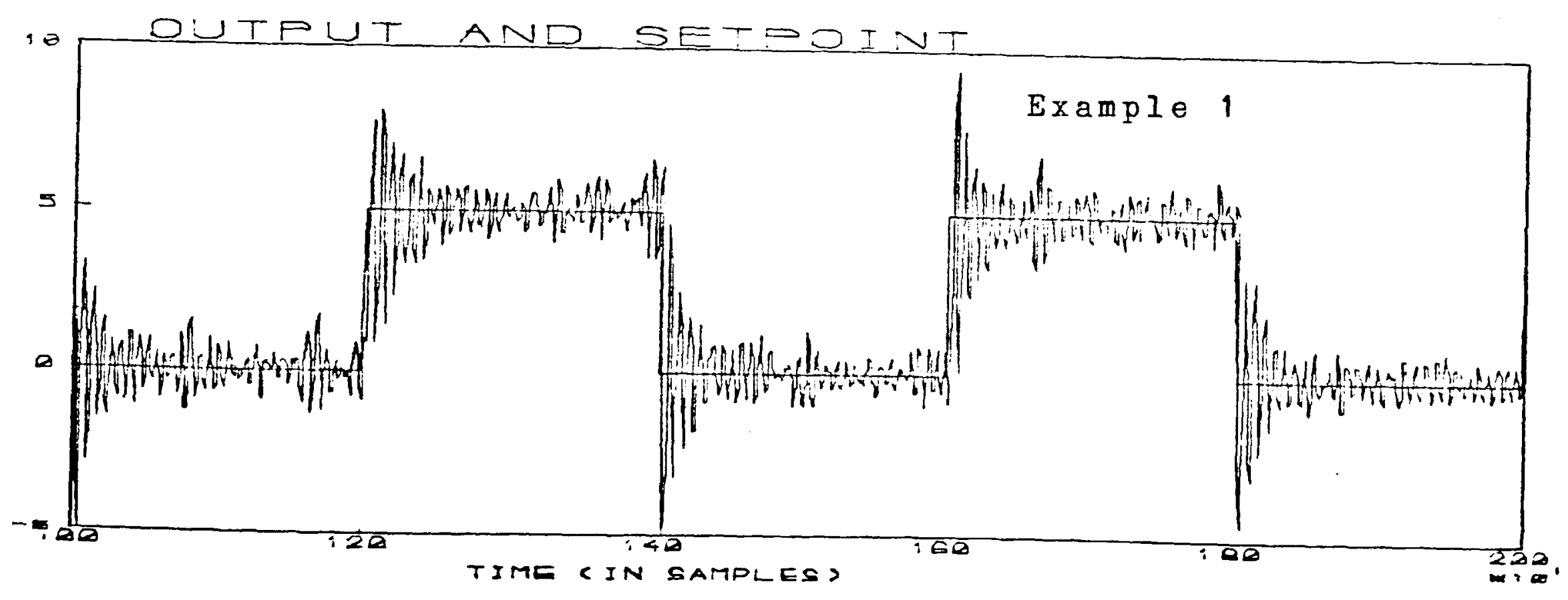

Fig. 5-2a Reduced order Controller with $P$ as for above 


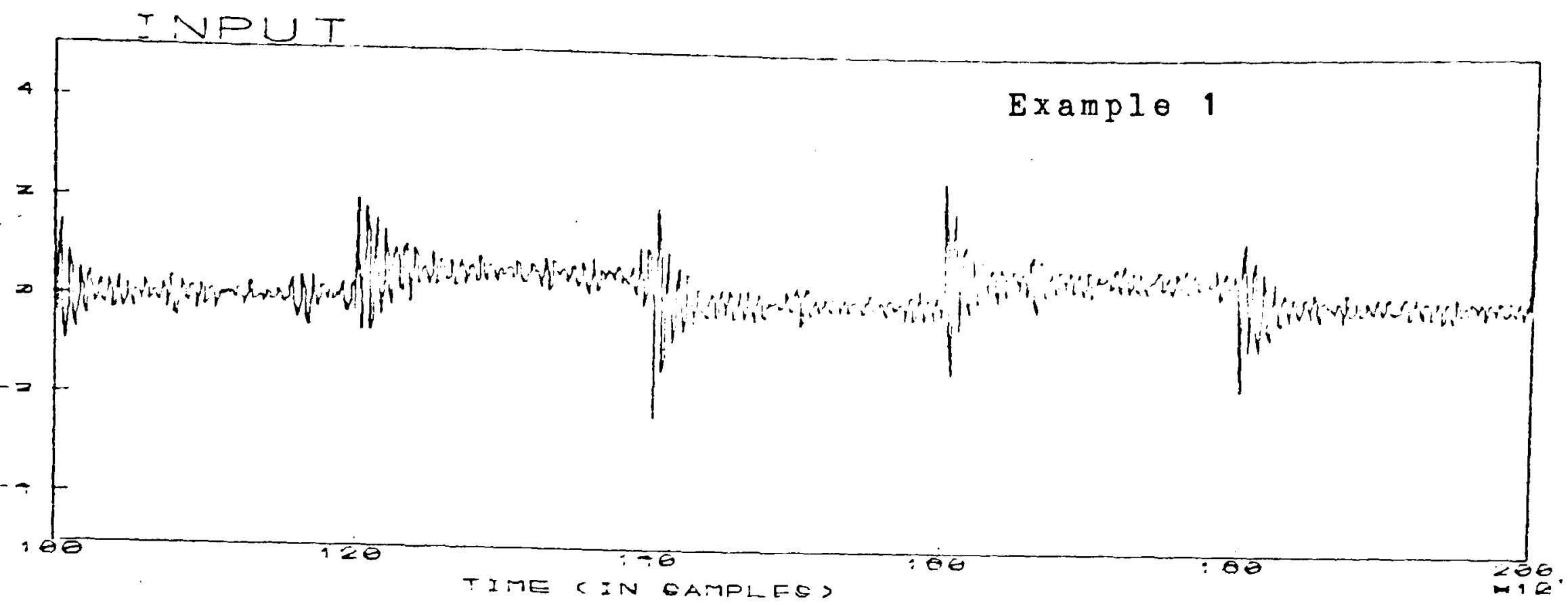

Fig. 5-2b Input from Peduced order Controller (cf Fig. 5-1b)

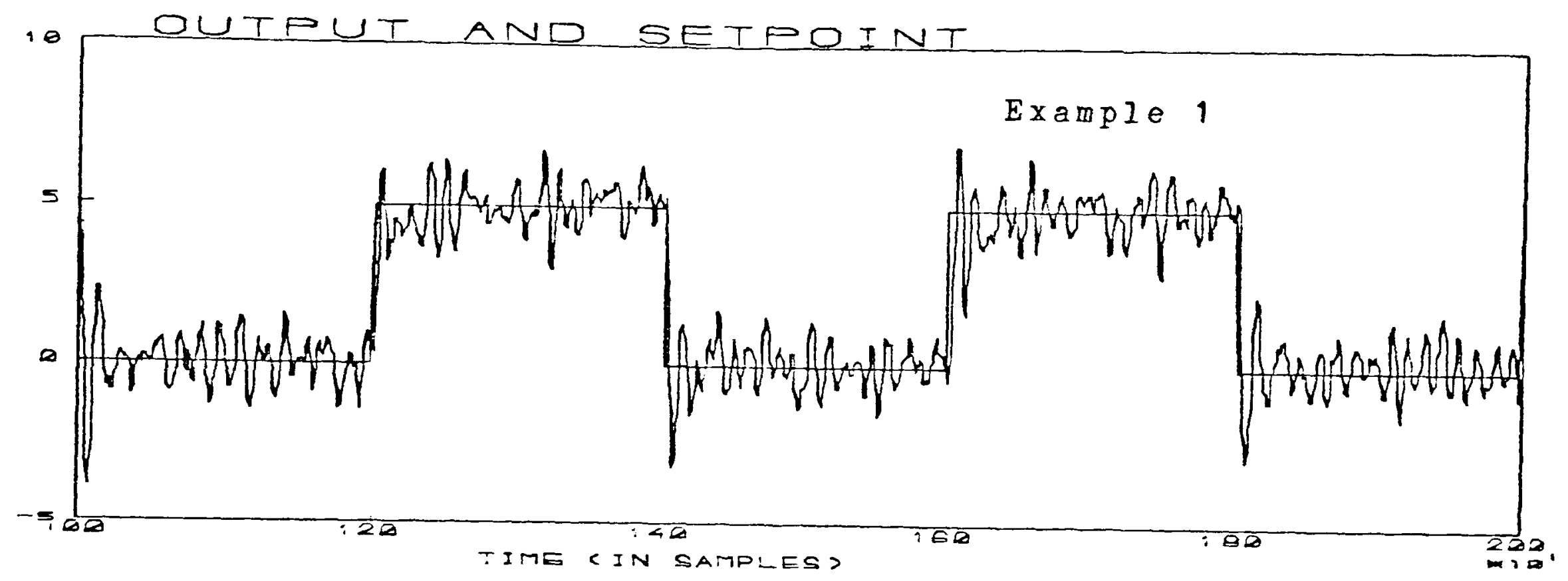

Fig. 5-3a Reduced order Controller with Slower P (cf 5-2a)

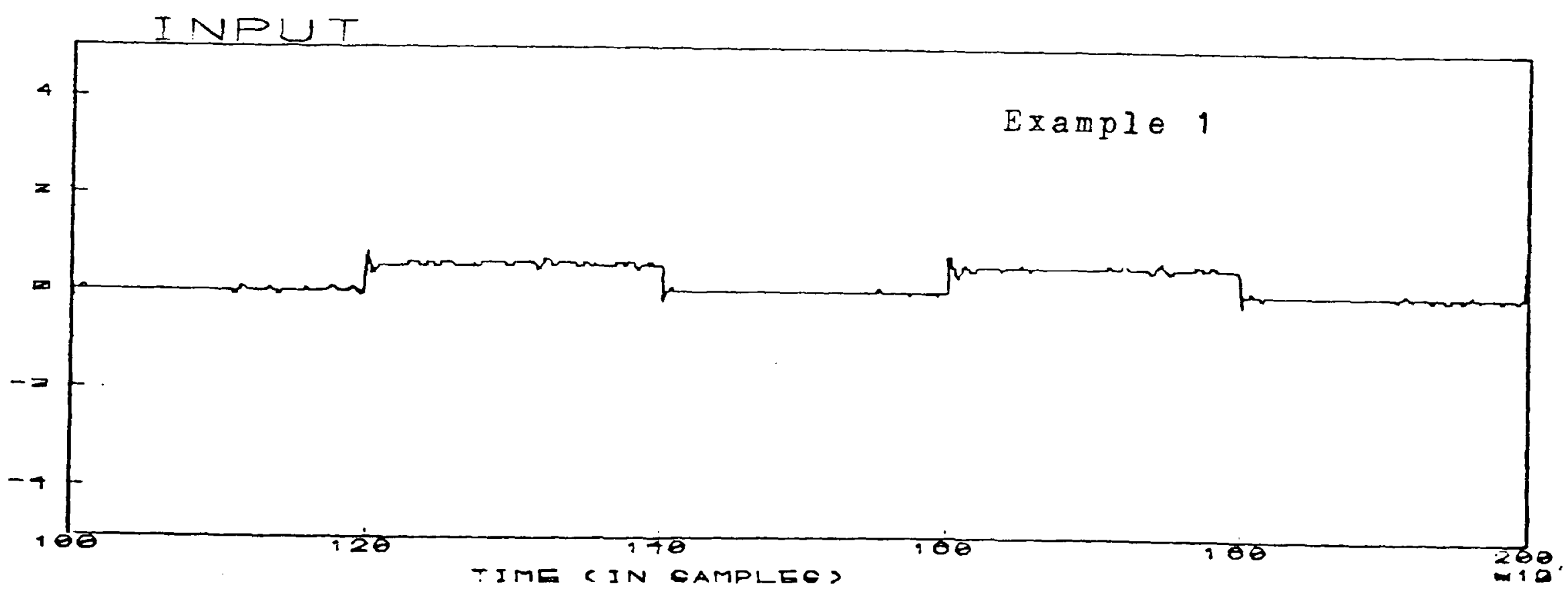

Fig. 5-3b Input from Reduced order Controller with slow 


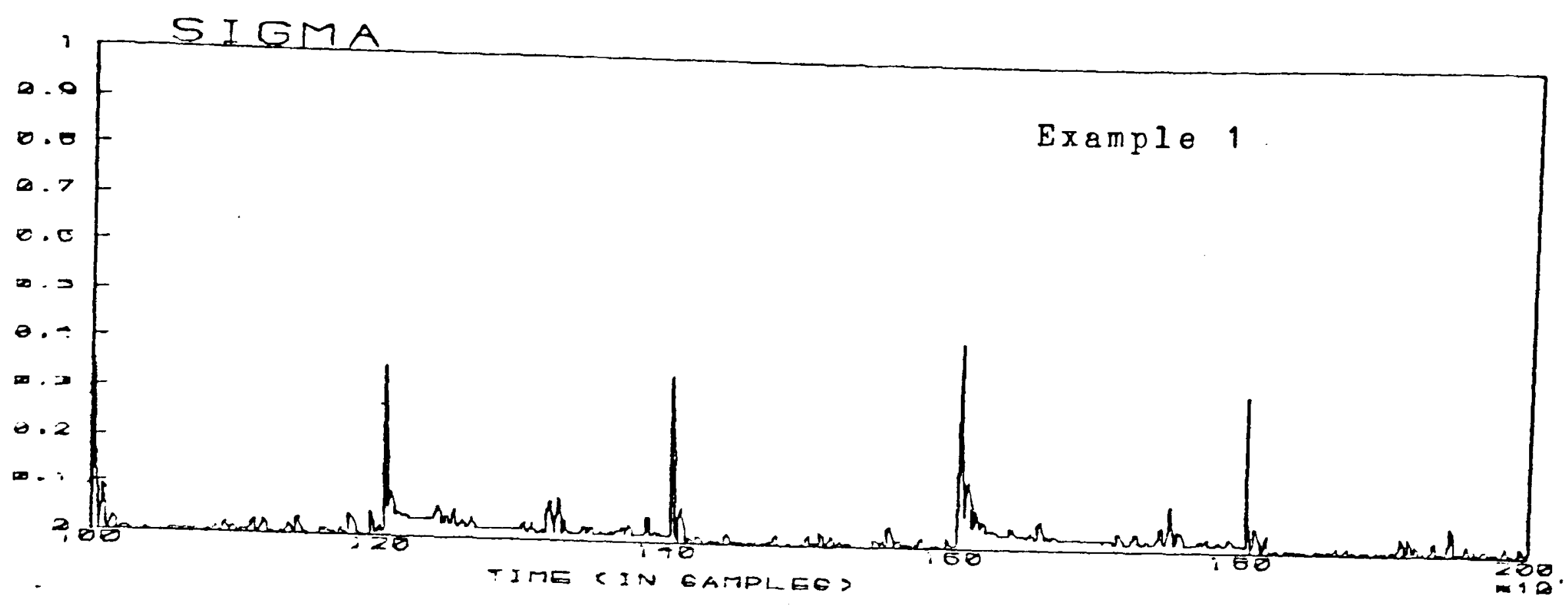

Fig. $5-3 c \quad \sigma(t)$ sequence for reduced order controller

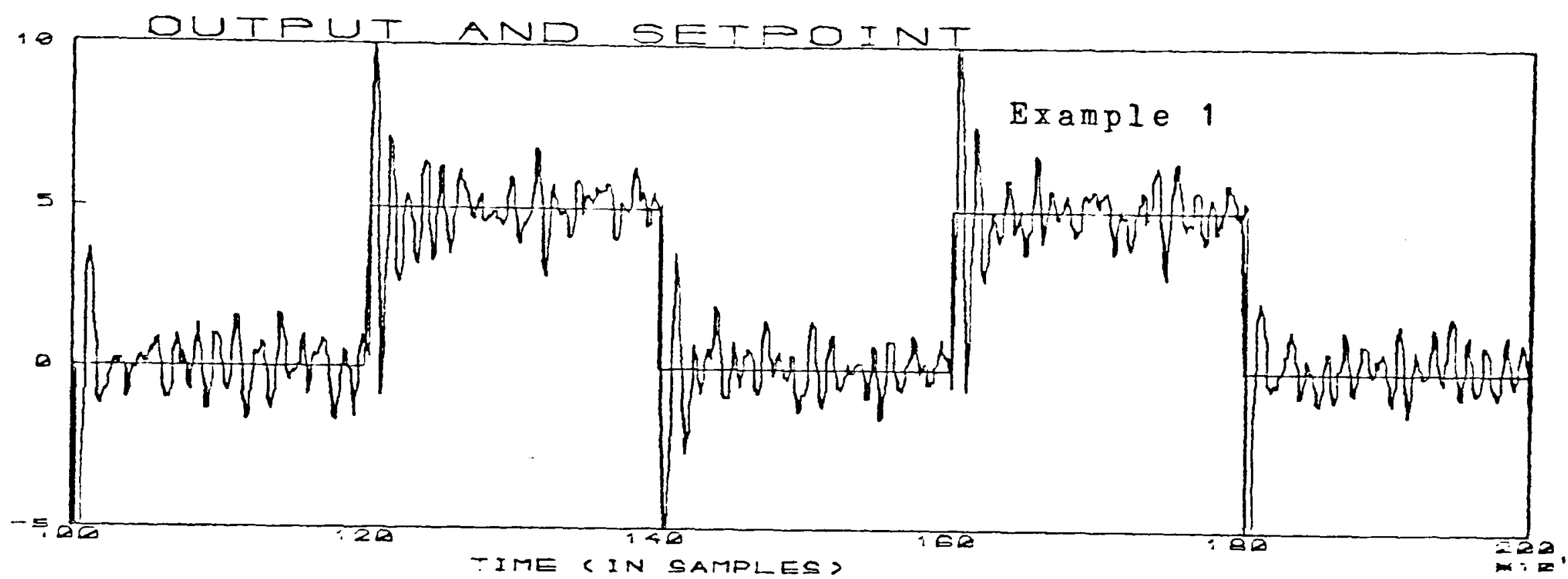

$\begin{array}{ll}\text { Fig. 5-4a Reduced Order Controller with slow Predictor pole } & \text { (cf Fig. 5-3a) }\end{array}$ INPUT

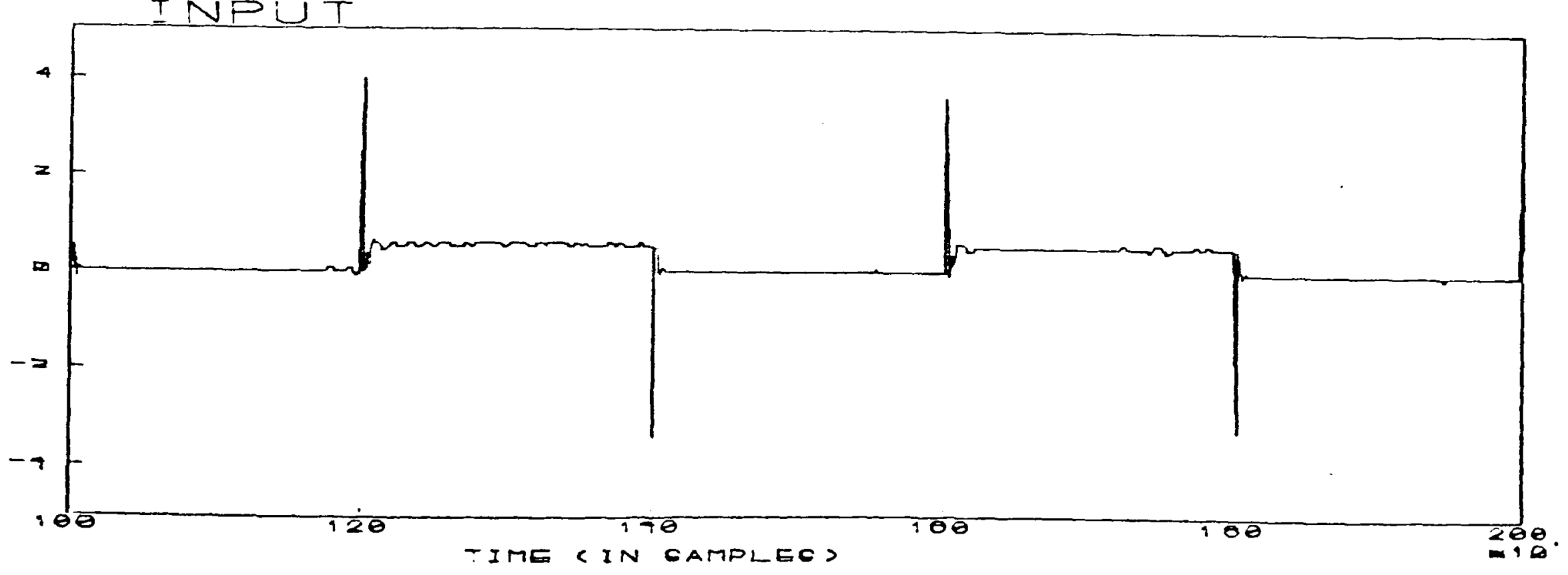

Fig. 5-4b Reduced order Controlier with slow Predictor pole (cf Fig. 5-3b) 
Full order, i.e. no. of $F$ parameters $=$ no. of $G$ parameters $=2$

$$
\begin{aligned}
& P\left(q^{-1}\right)=2\left(1-0.5 q^{-1}\right) \quad \text { : lst order reference model with } P(1)=1 \\
& Q\left(q^{-1}\right)=0 \quad \text { and } \quad C\left(q^{-1}\right)=1 \quad \text { :no control weighting and fast }
\end{aligned}
$$

predictor

Pig. 5-1 serves for later comparison. The output tracks the setpoint with no significant overshoot and the input is not "too active". Pigs. 5-2a,b show a simulation for the same system, with the same design polynomials $P, Q, C$ but with a first order controller i.e. no. of $P$ parameters $=$ no. of $G$ parameters $=2$. In section 3.4.3, stability was predicted for this system when such a controller is used. Not surprisingly however, the output is noisier than in the full order case and there is significant overshoot during setpoint changes. The sample variance of the estimated prediction error, $\hat{e}_{\mathfrak{l}}$, has also increased from 0.173 in the full order case to 2.42 in the reduced order case. The input too is noisier. Note however that Goodwin and Ramadge[45] have shown that the same system would have been unstable if $p$ had been selected to be 1 j.e. minjmum varjance control is used.

Fig. 5-3a shows the result of using a slower $P$, with a root at $q^{-1}=1.25$. As expected, the response to a step change in the setpoint is less oscillatory. There is also the benefit of a less 'active' control, as indicated in Fig. 5-3b. Fig. 5-3c shows a plot of the $\sigma(t)$ sequence over the time interval $1000-2000$. Other than the spikes due to step changes in setpoint, $\sigma(t)$ is small. This implies that the assumption of a small $\sigma_{0}$ in Chapter 3 is justified. 


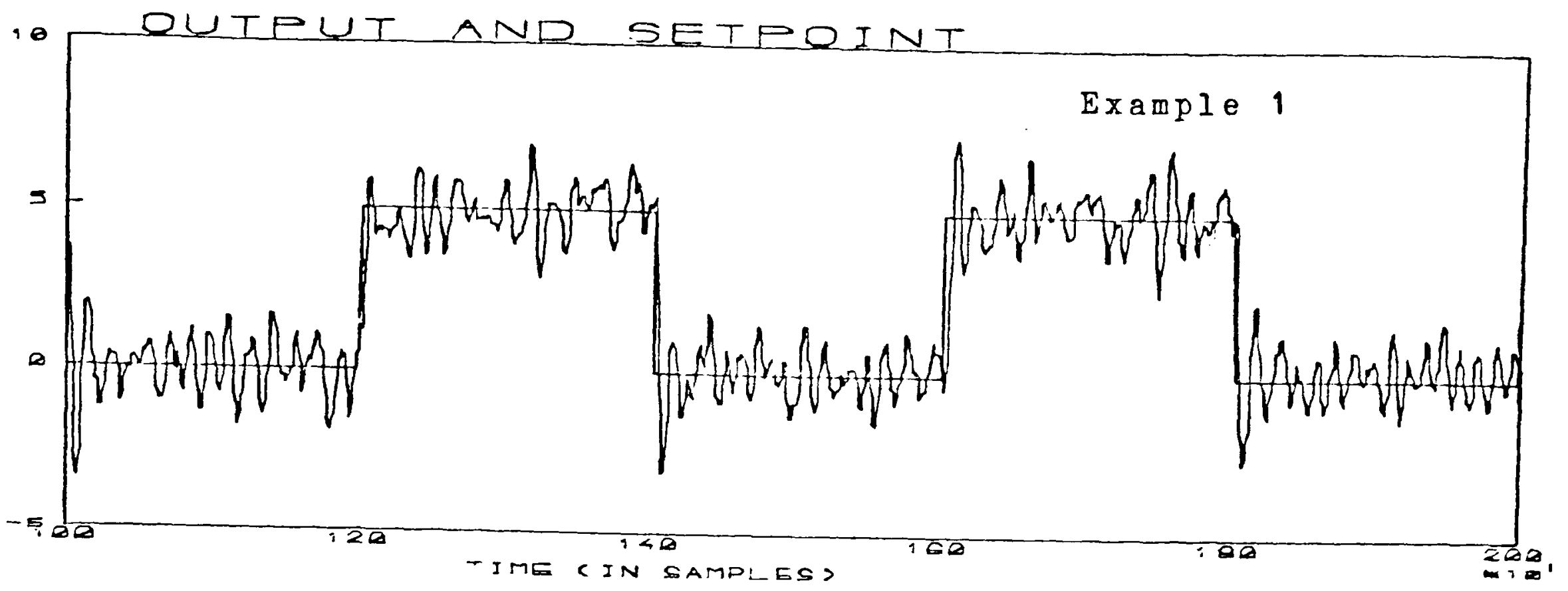

Fig. 5-5a Reduced order Controller with Scalar Gain Estimator

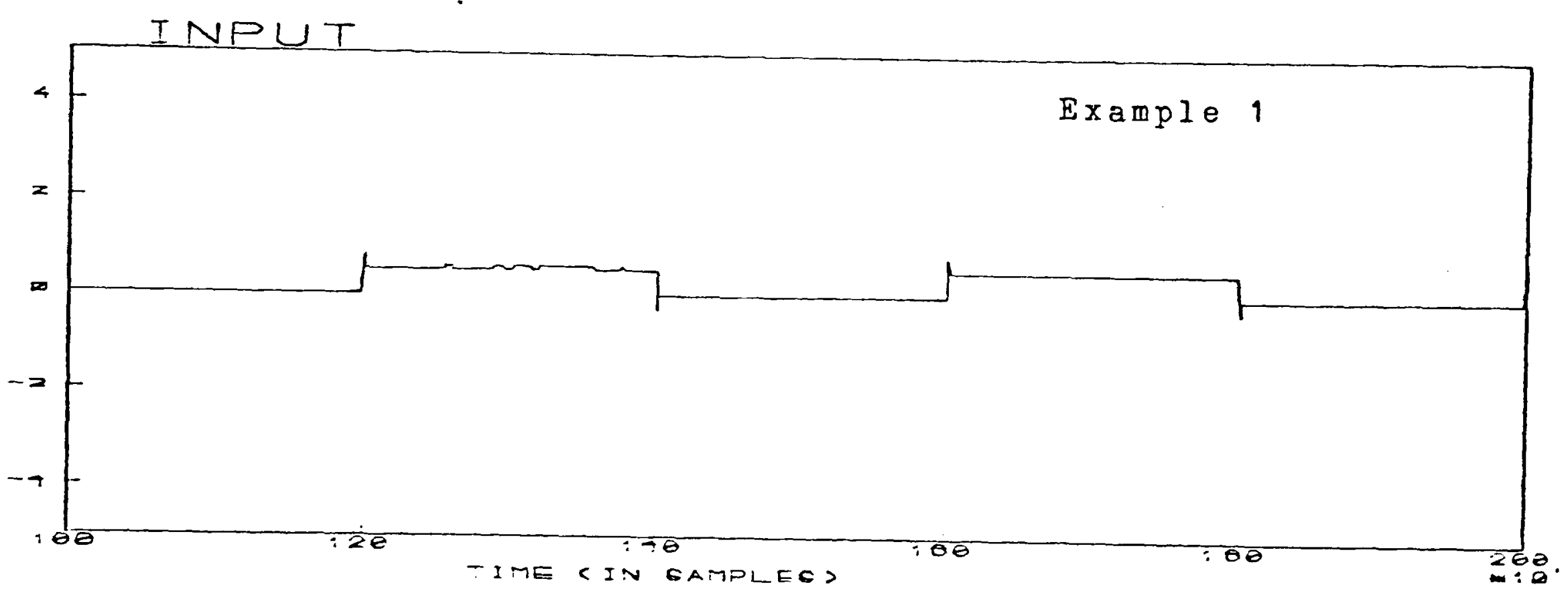

Fig. 5-5b Input when scalar gain estimator is used

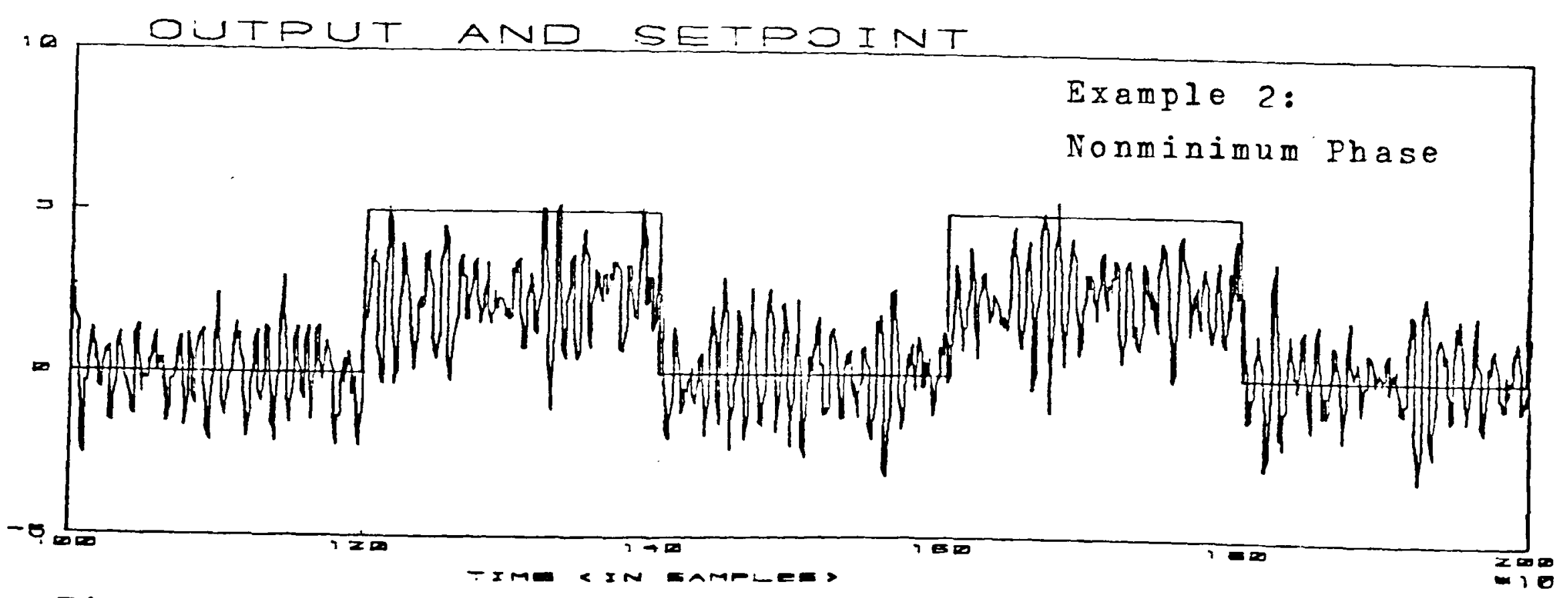

Fig. 5-6a Reduced Order Controller with $Q \neq 0$ 


\section{DF.STGN F.XAMPT.FS}

In Chapter 3, it was also shown that as far as robust stability is concerned, a slower predictor/observer, $\frac{l}{c}$ would serve as well as a slower model, $\frac{l}{p}$ (assuming that $Q=0$ ). Fig. 5-4 shows a simulation run with a predictor pole at $q^{-1}=1.2 .5$ and with model $\frac{1}{p}=1$. With a slower predictor, $\hat{e}_{1}$ has a smalier variance but the output is highly oscillatory as minimum variance control is attempted on the predicted model. The input is also less satisfactory because of the large spikes at setpoint changes.

The run of Fig. 5-5 uses the same controller as that of Fig. 5-3, except that a scalar gain estimator as described in section 3.8 is used. The controller was a great deal longer settling down from the same initial conditions, but once it did, it gives virtually the same performance as indicated in the figure.

These simulations illugtrate some of the results of the previous chapters. In particular, note that as assumed, $\sigma(t)$ is small, despite the use of a reduced order controller. It is possible to design a stable reduced order controller using the $P$ design polynomial. Furthermore, a slower model is shown to be more robust in that a less oscillatory output is obtained. A slow predictor $(C)$ is also shown to give robust stability but the resulting control performance is less satisfactory.

Some simulation runs for the same system when the additional problem of non-zero mean disturbances is considered will be shown after the next example. 


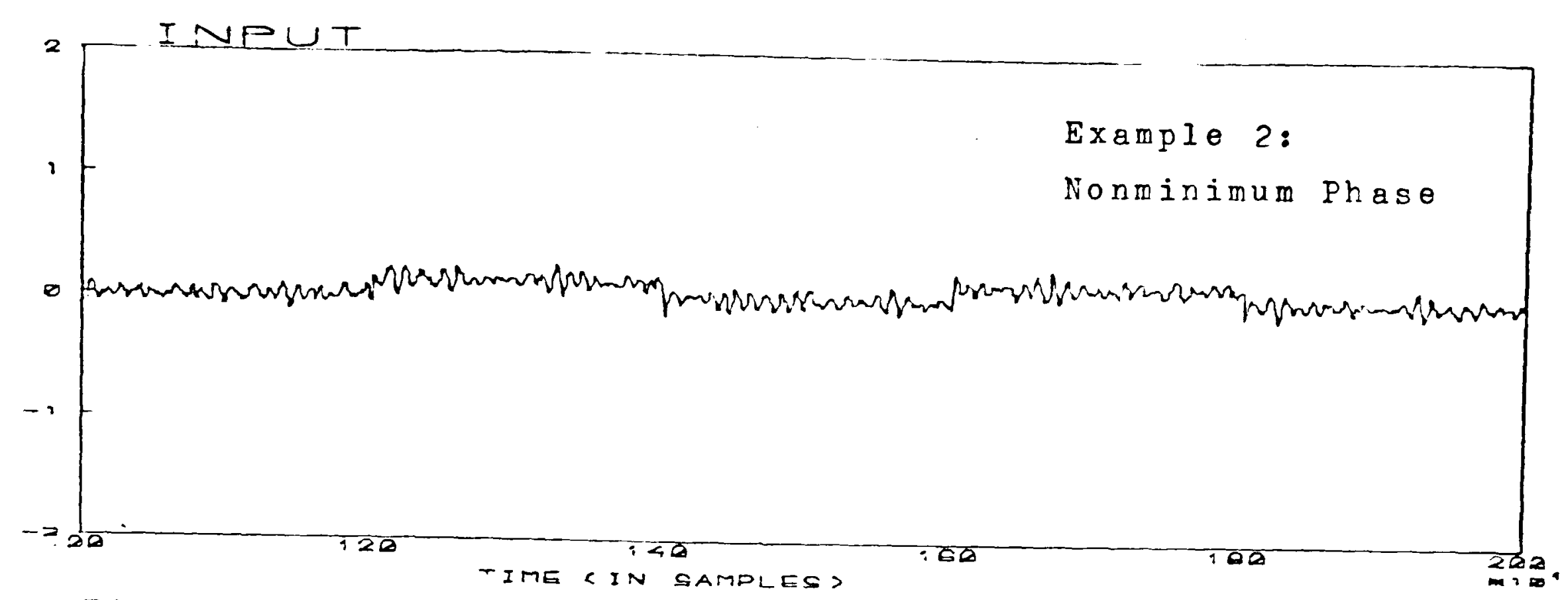

Fig. 5-6b Input from $Q$ weighted, reduced order controller

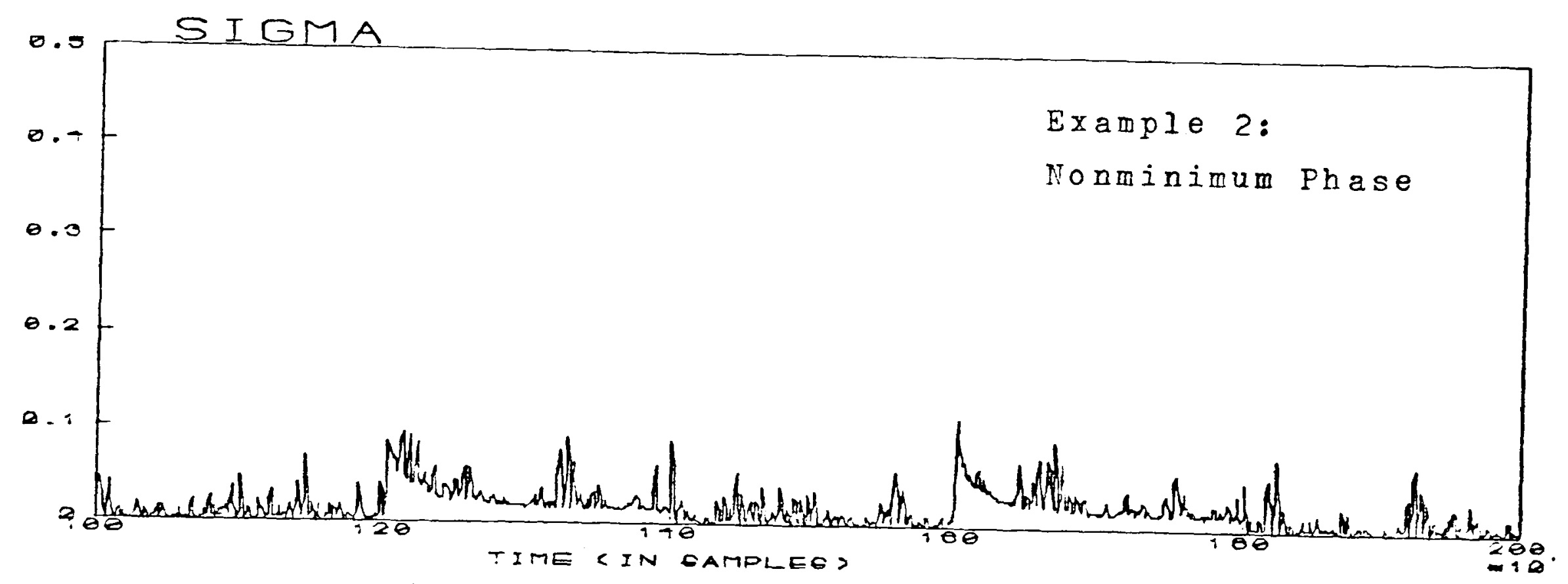

Fig. 5-6c $\sigma(t)$ sequence for Nonminimum Phase system

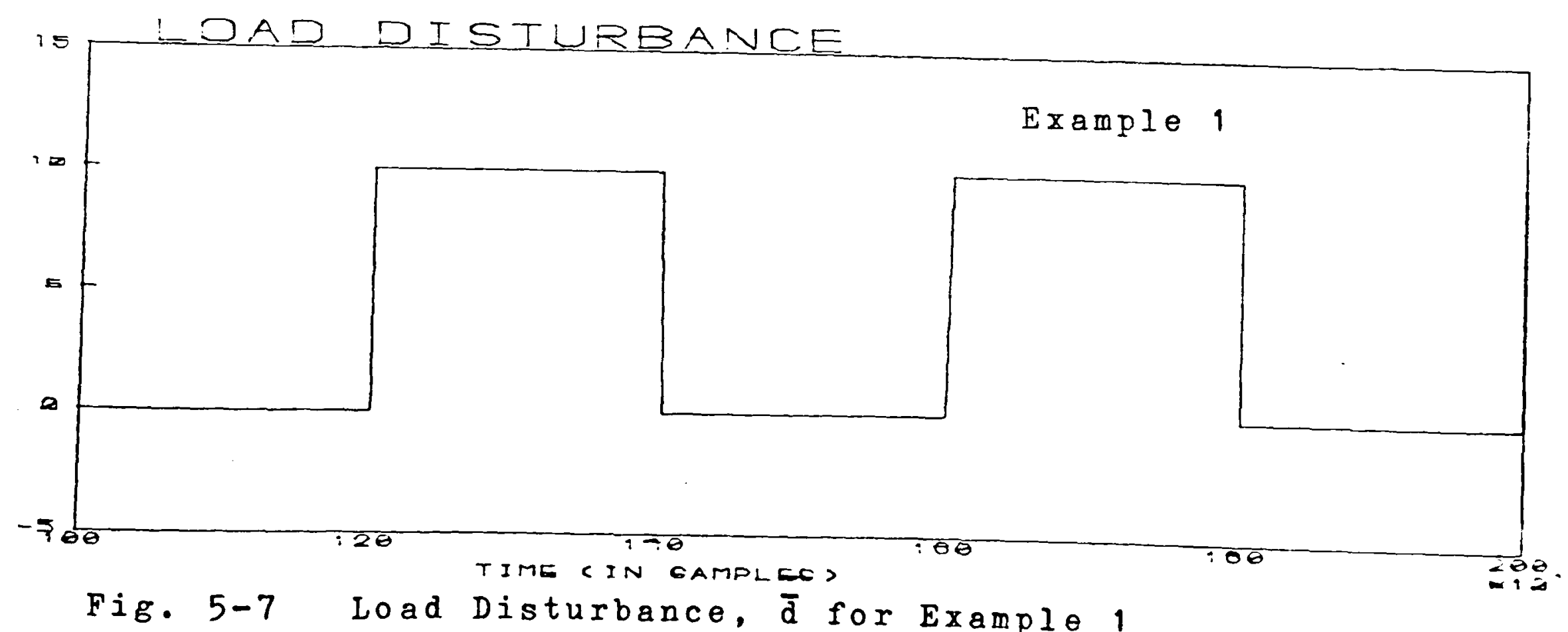


We consider a system with the same poles as the system of Fxample $r$, but wi.th a nonminimum phase zero i.e

$$
B_{D}=1.6+2.0 q^{-1}
$$

From Chapter 3, we know that the generalised control weighting, the $Q$ polynomial must be selected so that $P B{ }_{0}{ }^{Q A}{ }_{0}$ has stable roots. Chapter $I$ indicated that $Q$ must also be sejected so that $Q(1)=0$ to ensure that there is no steady state tracking error when the full order controljer is used.

Since $A_{0}$ will not usually be known, then assuming that it is stable, a safe choice of $Q$ is to have a large weighting factor, such as

$$
Q=2.0\left(1-q^{-1}\right) /\left(1-0.99 q^{-1}\right)
$$

This choice of $Q$ corresponds to a classical PI compensator on the predicted tracking exror, with a 'low' gain.

Fig. 5-6 shows the results of a simulation run with this choice of Q. when the controller is low order (as in Fxample 1). Note that when the setpoint is not zero, there is a significant tracking error and the output is rather noisy. If steady state error is important, one of the schemes discussed in Chapter 4 for offset removal will have to be used.

The disturbance attenuation is very poor, but this is not surprising because of the low gain of the $\frac{1}{Q}$ compensator. Fig. 5-6c shows that the $\sigma(t)$ sequence is also small.

Hence $Q\left(q^{-1}\right)$ can be used to stabilise a nonminimum phase system even in the reduced order case. Used by itself, the result is rather poor control when the setpoint is non-zero. 


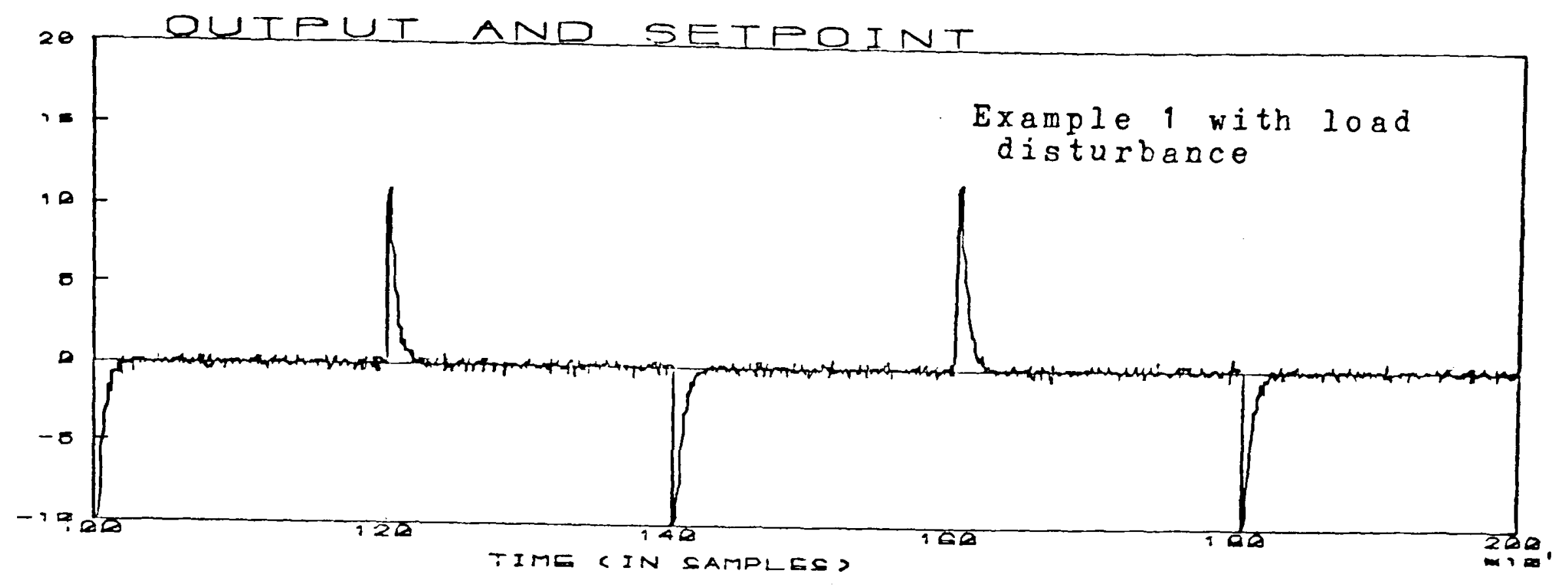

Fig. 5-8 Full order Controller with Incremental Predictor

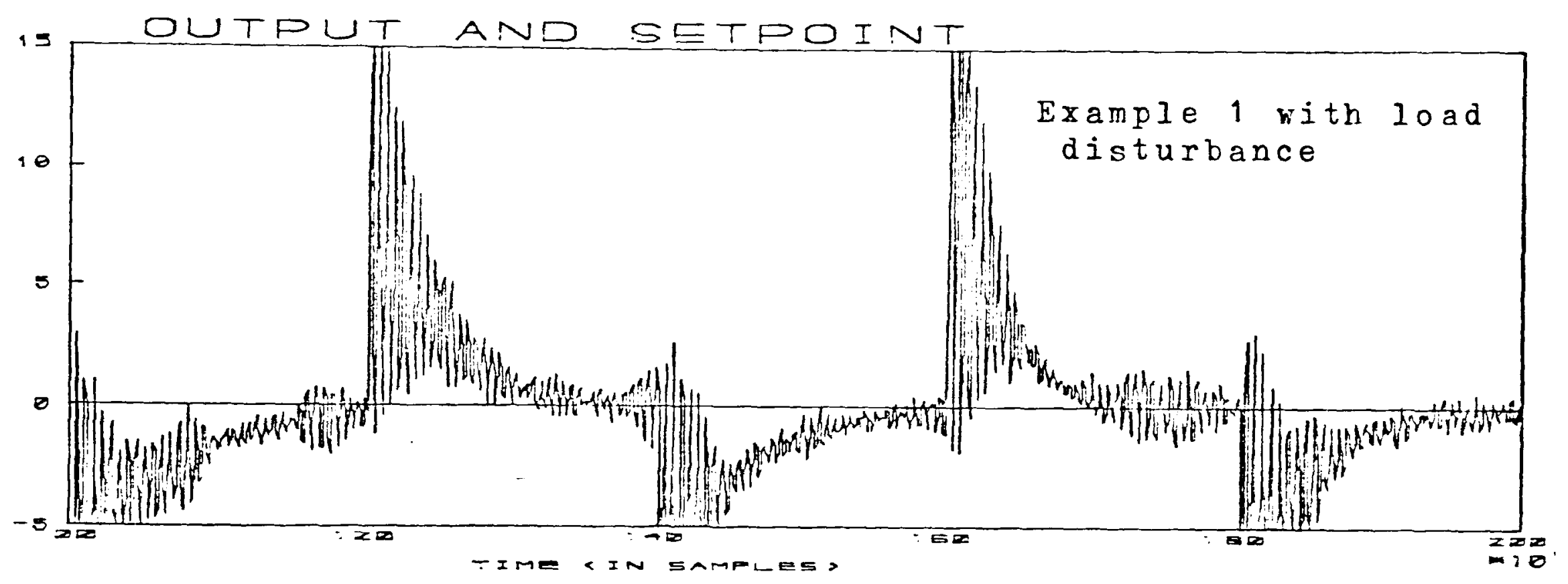

Fig. 5-9a Reduced order Controller with separate Pias Est.

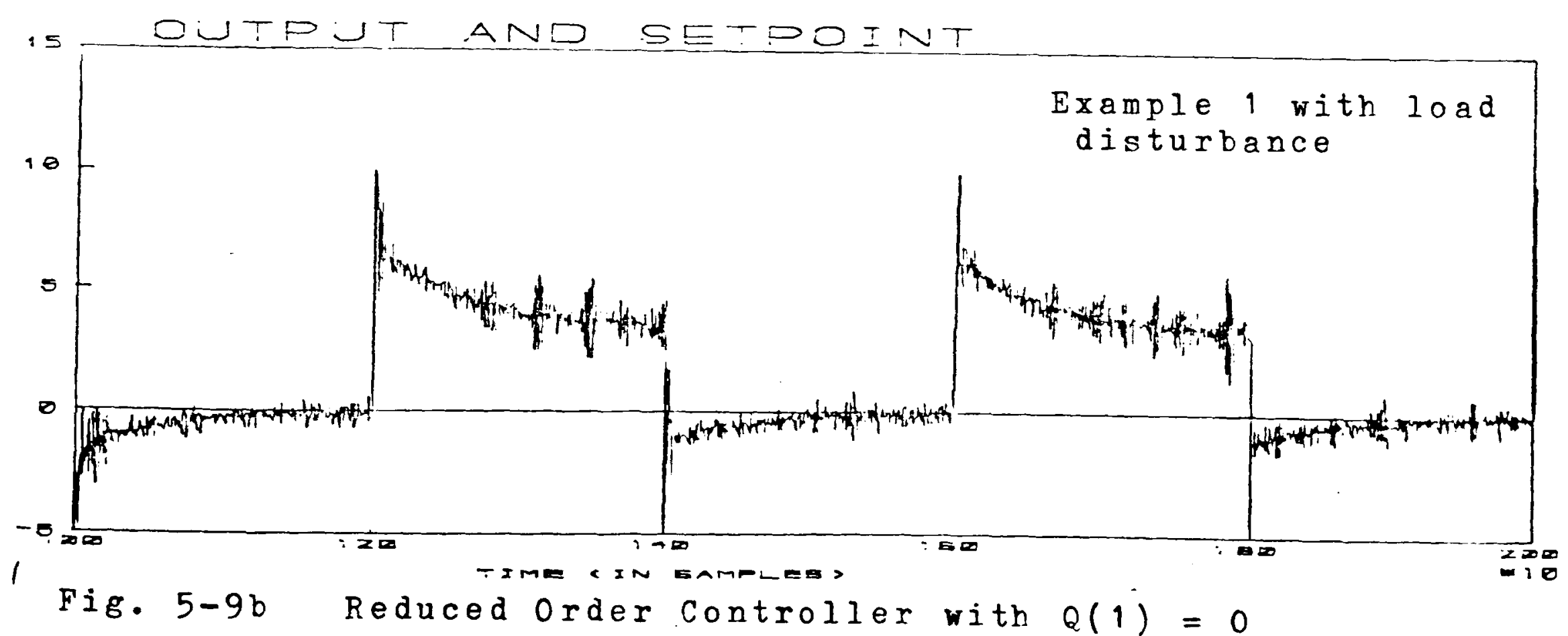




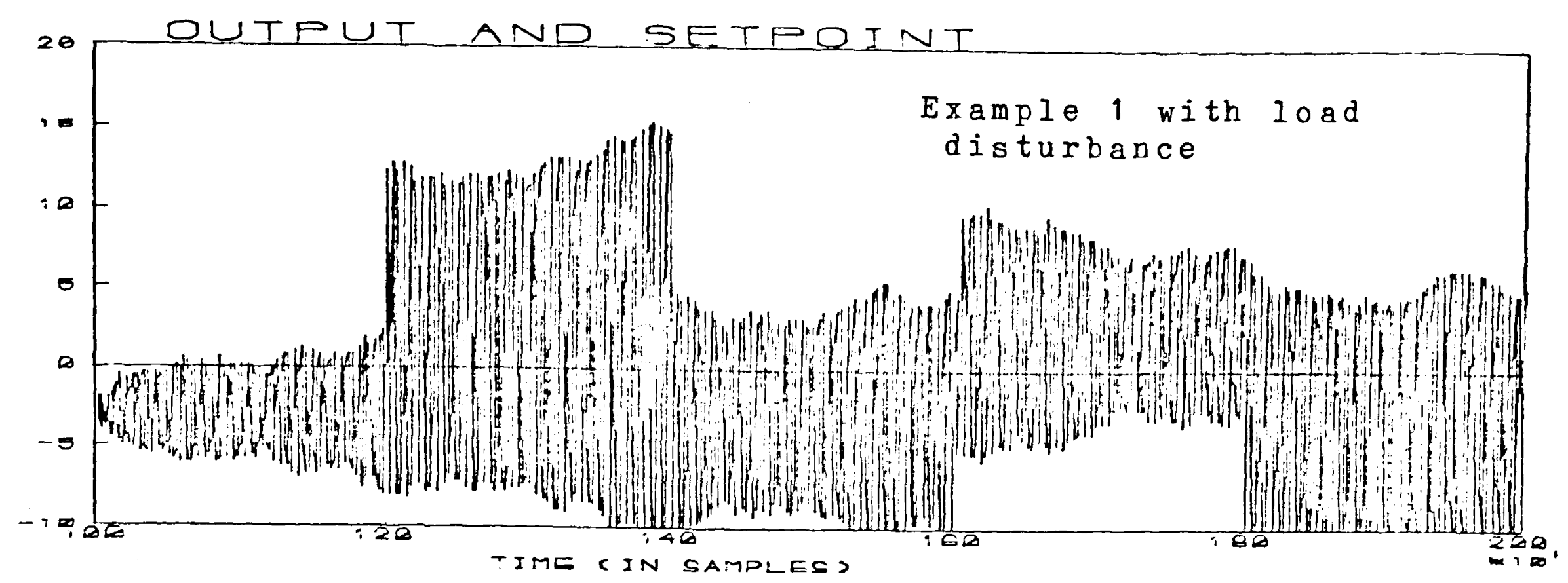

Fig. 5-9c Reduced order Controller with Incremental Predictor

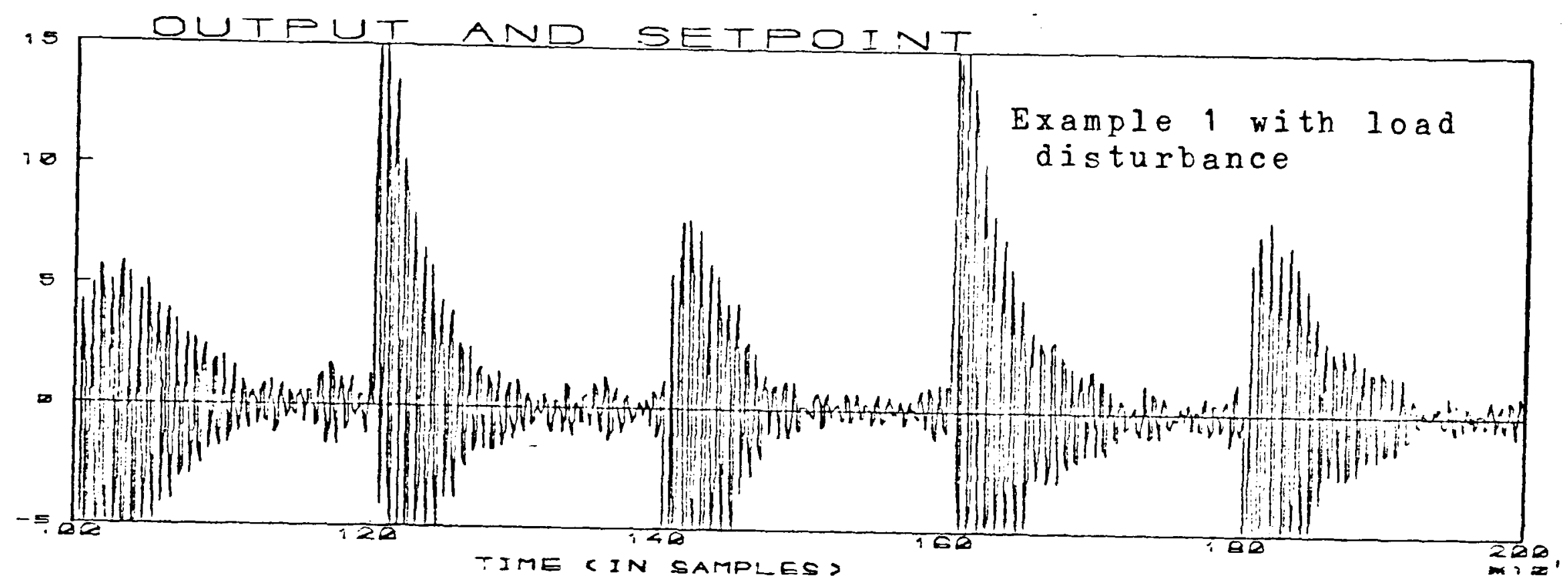

Fig. 5-9d Reduced order Controller with outer Loop PI

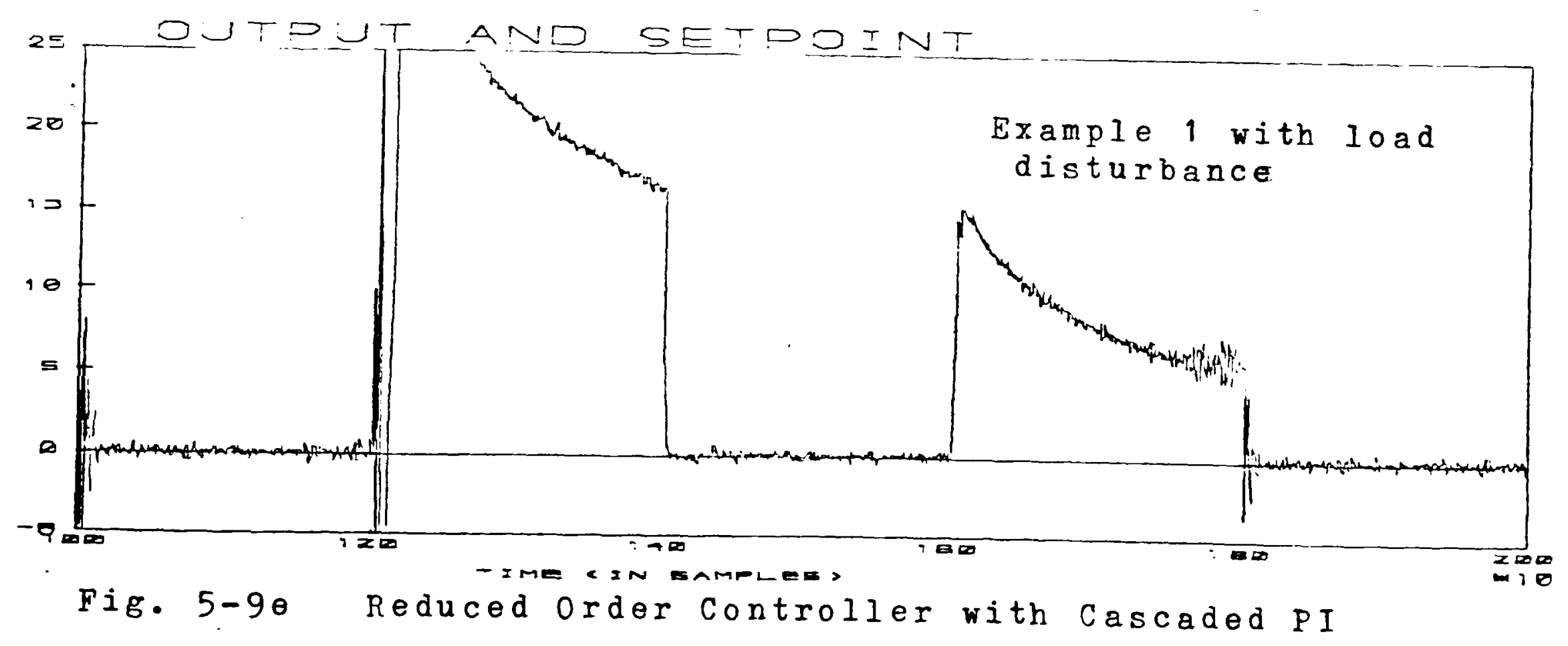




\section{DF.STGN F.XAMPT.F.S}

\section{Fixample 2 (Continued) (Offset Filimination)}

In F.xample 2, we noted that in the reduced order case, there is a tracking error even when the generalised control weighting, $Q$ is sejected to have rero steady state gain. In Chapter 4, some methods for offset ejimination proposed in the jjterature were studjed. Here the system and reduced order controller of fixample J wj]J be sjmulated, when the system is subjected to the load disturbance, $\bar{d}$ of Fig. 5-7.

To establish a basjs for comparjson, Fig. 5-8 shows the output when a fulj order controller is used with the incrementaj predictor discussed in section 4.3. Despjte the presence of noise, the load disturbance rejection is very good. Figs. 5-9a..e show the resujts of using a reduced order controller with each of the following offset elimination methods.

Fig. 5-9a: a separate bias estimator, cf section 4.3 .1

Fig. 5-9b: generalised control weighting, with

$$
Q=0.1\left(1-q^{-1}\right) /\left(1-0.98 q^{-1}\right)
$$

Fig. 5-9c: incremental predictor, cf section 4.3.1a

Fig. 5-9d: Outer Joop PI, cf Section 4.3.1C

Fjg. 5-9e: Cascaded PJ, cf Section 4.3.1b

In the last two cases, the parameters of the PJ compensator were selected by trial and error to give approximately the 'best' response. In all cases, the reference model was selected to be $p=5.0\left(3-0.8 q^{-1}\right)$.

Fig. 5-9c shows that in the reduced order case, the incrementa] predictor method is very poor. Fig. 5-9b confirms that choosing $Q(J)=0$ wil] not remove the offset when the load disturbance has a non-rero mean. When there is no Joad disturbance, jt does remove offset, even in the 


\section{DF.SIGN F.XAMPTIFS}

reduced order case.

Fig. 5-9a shows that for this simulation of a linear system, the method of separate biag estimation does work; here, with a bias forgetting factor of 0.95 compared with 0.99 for the other parameters.

The cascaded PI method illustrated in Fig. 5-9e compares rather unfavourably with the other methods, but it does appear to give a less noisy output. Fig. 5-9d shows that the outer loop PI removes the offset quite well, but at the cost of a noisier output.

Comparing the Fig. 5-9 plots with Fig. 5-8, it is clear that a reduction in controller order in the presence of load disturbances results in significant deterioration of controller performance for all the methods considered. The reduced order incremental predictor is the worst. For this example, the method of outer loop PI is the quickest to remove the offset due to load disturbance.

\section{Fxample 3 (Pole Placement)}

An often quoted advantage of the pole placement self-tuners discussed in Chapters 2 and 3 is that they can control nonminimum phase systems. However in section 3.6. it was shown that this controller may not be robustly stable. Here the example of section 3.6 .2 is simulated. First we consider a minimum phase system under pole placement control i.e

$$
\begin{aligned}
& A_{0}=1-1.7 q^{-1}+0.72 q^{-2} \\
& B_{0}=1.0-0.7 q^{-1} \\
& k=1 \\
& d(t)=\text { rero mean disturbance of variance } 0.5
\end{aligned}
$$




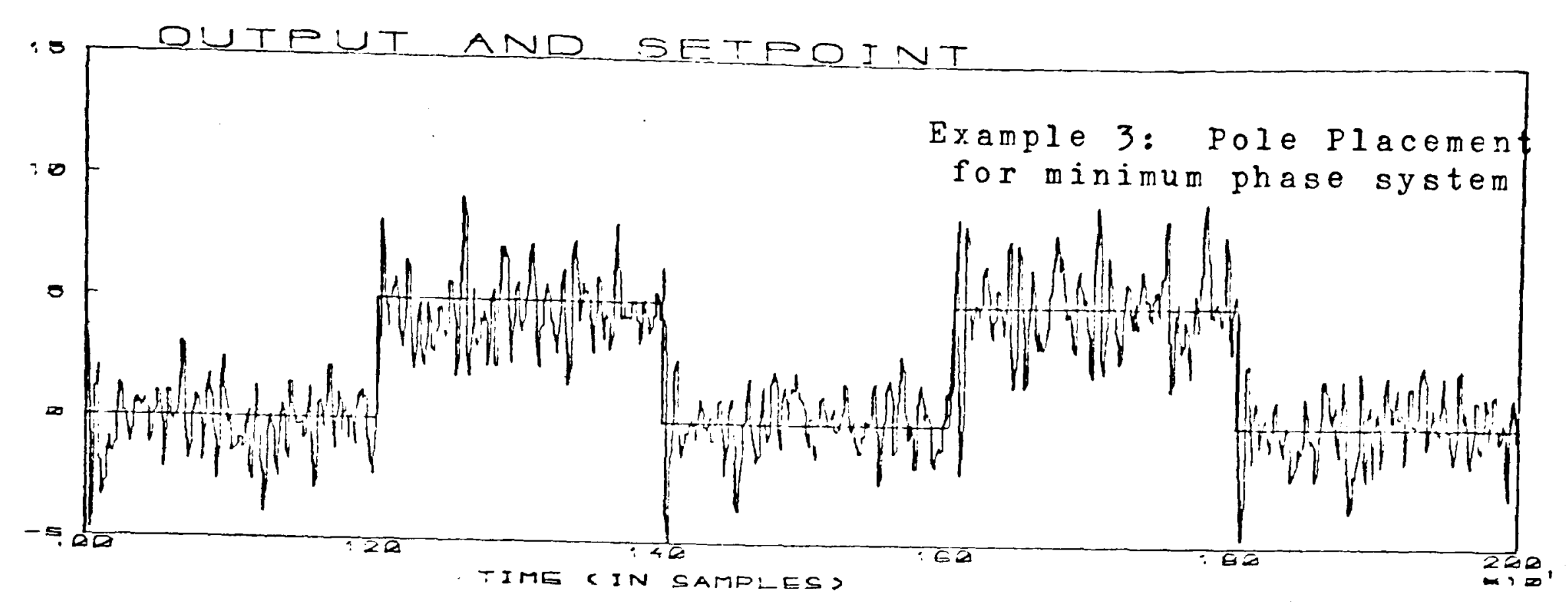

Fig. 5-10a Reduced order Pole Placement self-tuning

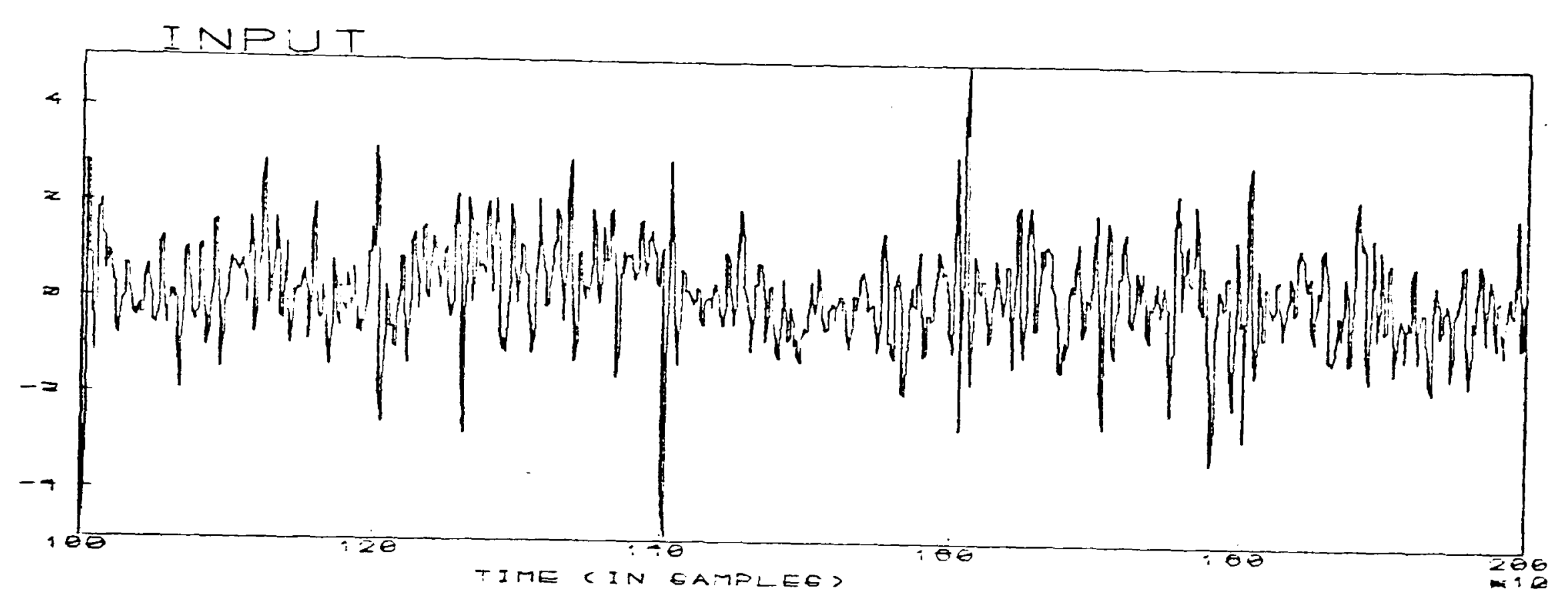

Fig. 5-10b Input from Pole Placement Controller

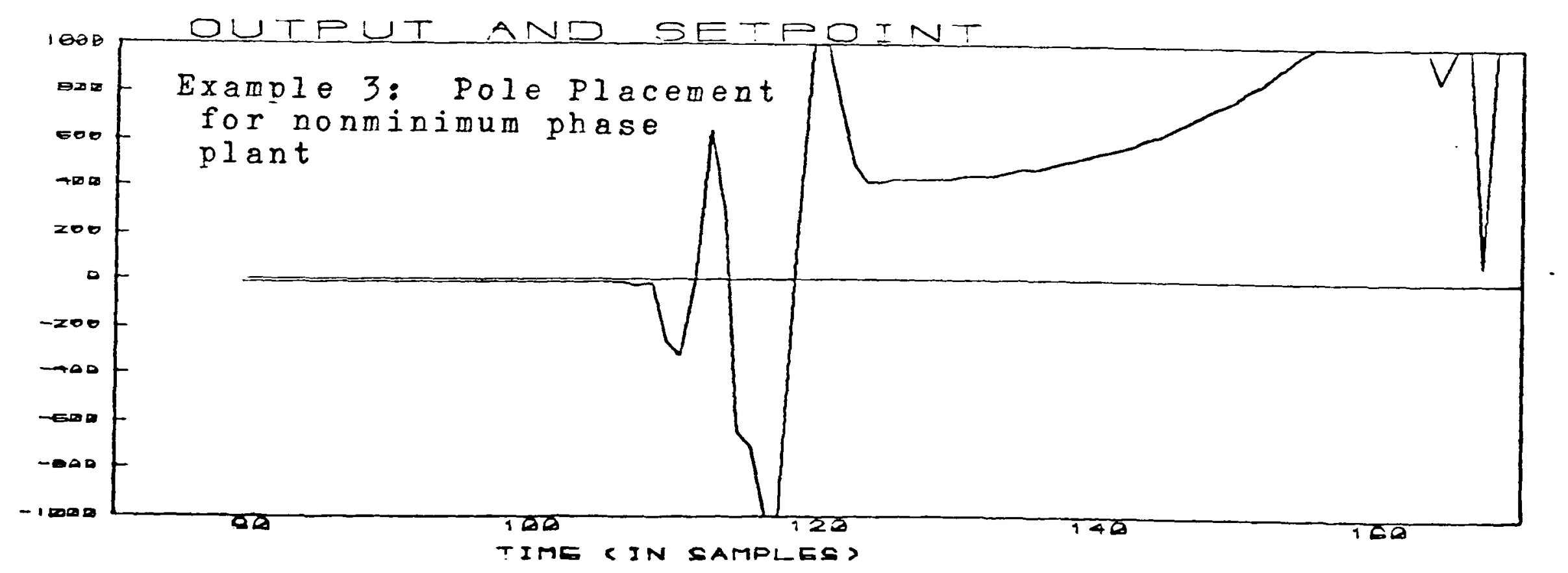

Fig. 5-11a Instability of pole placement self-tuning 


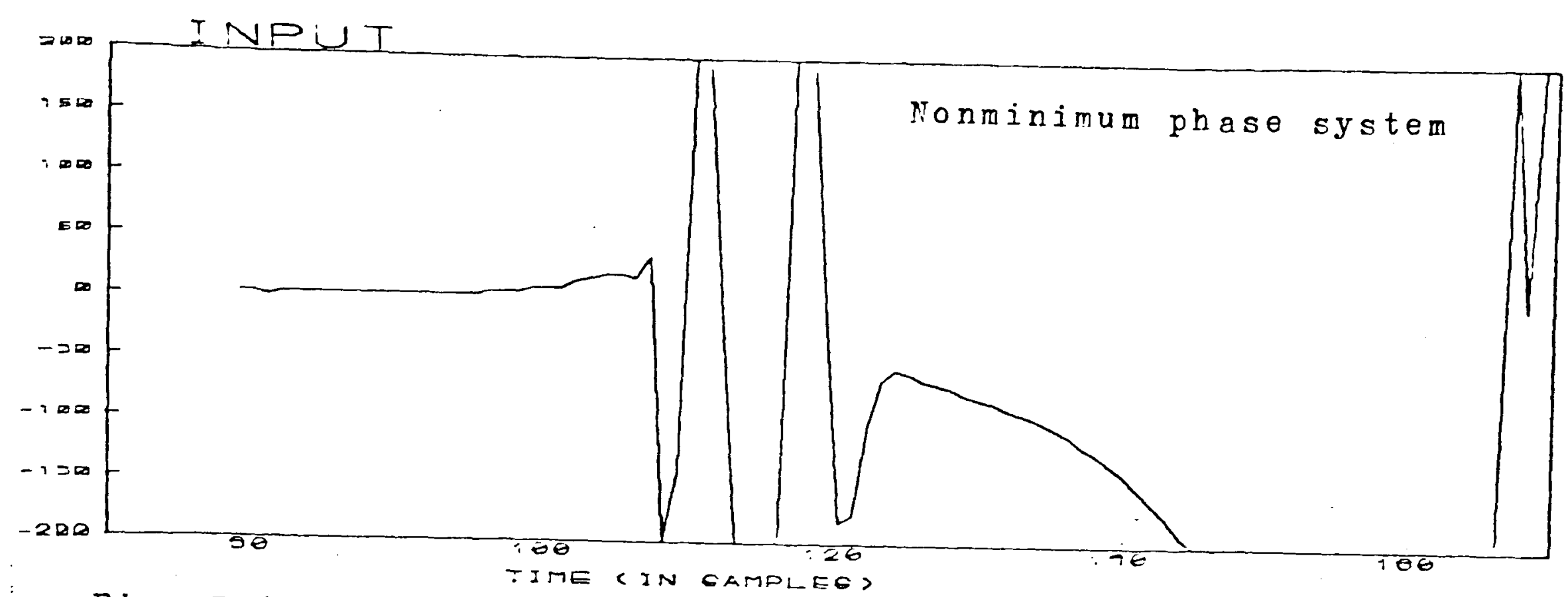

Fig. 5-11b Pole Placement self-tuning of Example 3

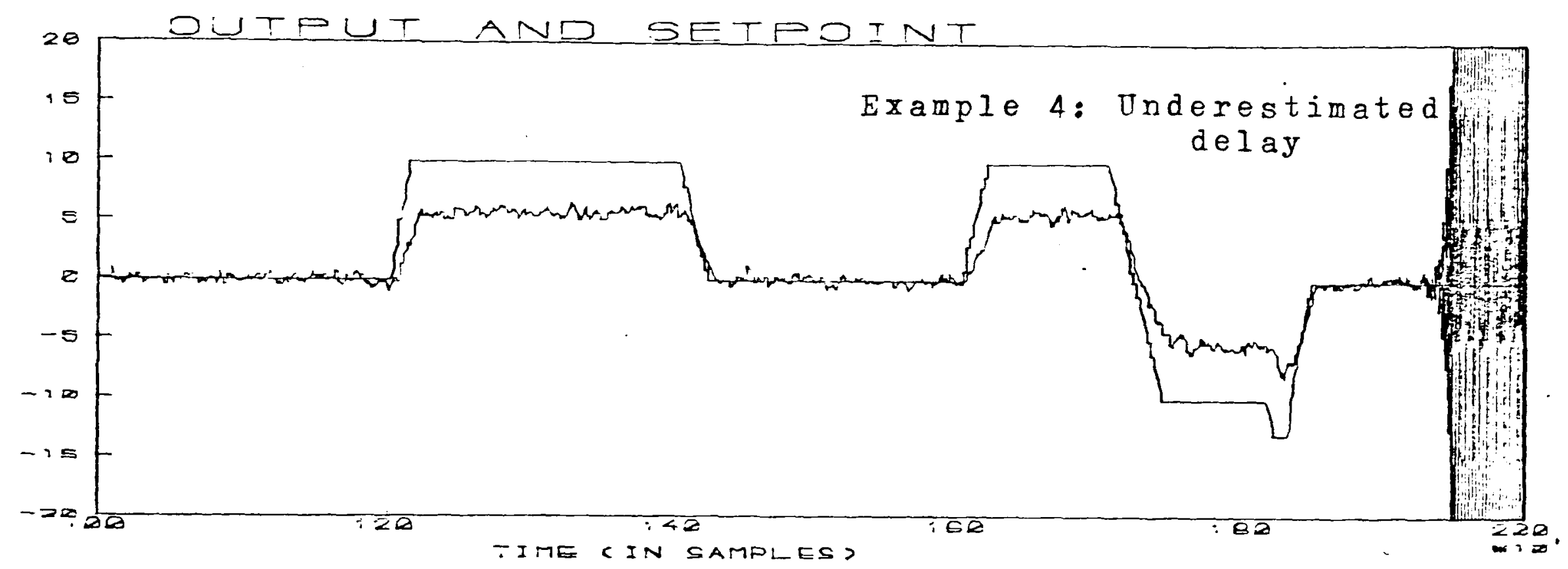

Fig. 5-12a Use of $Q$ to ensure stability

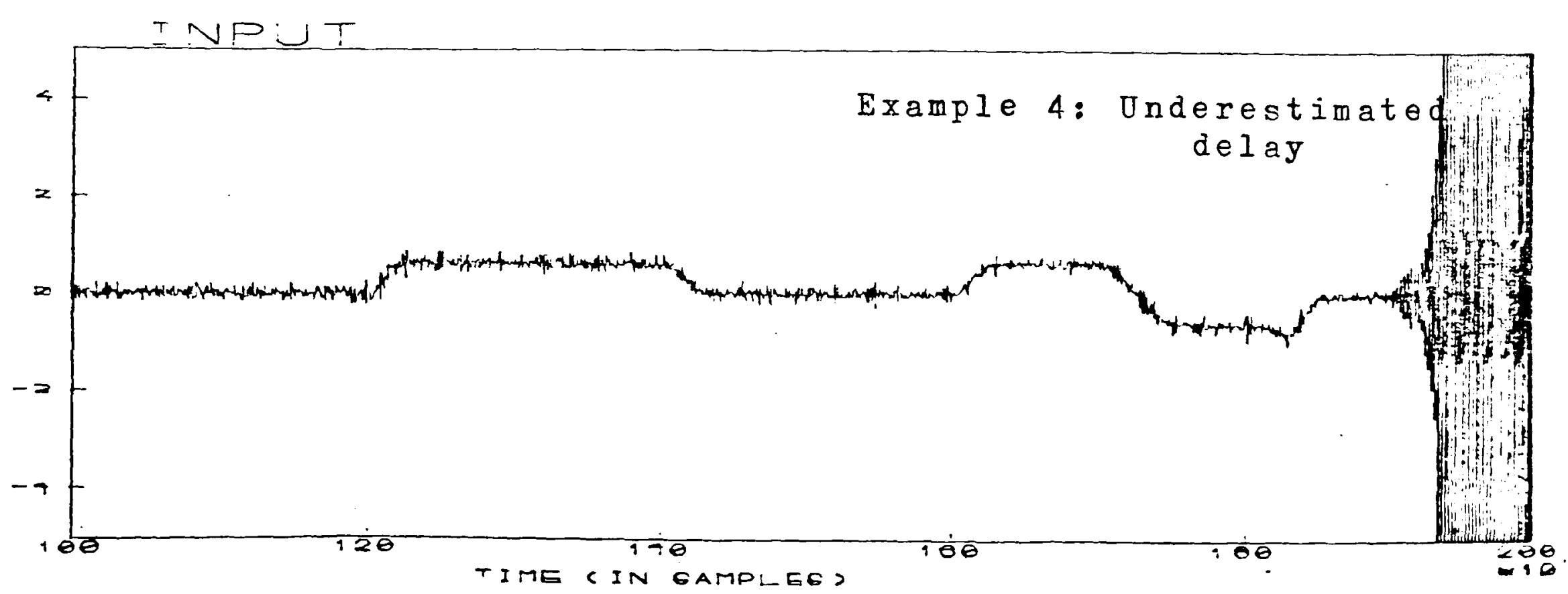

Fig. 5-12b Input from Full order Controller 


\section{DF.STGN F.XAMPT.F.S}

The pole placement controller is designed as if the system was first order, with the desired pole at $q=0.75\left(A_{m}=2-0.75 q^{-1}\right)$. In Section 3.6.2. it was predicted that the closed joop system would be stabje. Fjg. 5-jo julustrates this with the plot of output, setpoint and input for one simulation run from time $=1000$ to 2.000 . Despite the reduced order, the control is quite good and there is no offset.

When the system is reajly nonminimum phase $j . e$.

$$
B_{0}=1.0-1.2 . q^{-1}
$$

section 3.6.2. predicted instability. This too is confirmed in Fig. 5-11. Hence this simulation confirms that the pole placement controller is not robustly stable when the order is underestimated.

\section{Example 4 (Underestimated Delay)}

In this example, we consider the same system as Fxample 1 , except that the integer delay, $k=2$. Note that this is a minimum phase system. Assume that a full order controller is used, but with an assumed delay of 2. Section 4.2 says that the closed loop system would not be well-posed if $Q=0$.

If $Q \neq 0$, then from section 4.2.1, a sufficient condition for stabj]ity is that there should exist a nominal system $\frac{B}{A}$ such that

$$
\text { gain of } H\left(q^{-1}\right)=\frac{E\left(A_{0} B-q^{-1} A B_{0}\right)}{\left[\left(q^{-1} P B_{0}+Q A_{0}\right)\right.}<1
$$

where $B_{0}, A_{0}$ are as defined in Fxample 1 . In this example, the effective numerator polynomial of the true system is $q^{-1} B_{0}$. In this case, take $B=B_{0}$ and $A=A_{0}$ and let the design parameters be selected as

$$
c=1 \quad \text { :a very fast predictor }
$$


DF.SIGN F.XAMPI.F.S

$P=5\left(2-0.8 q^{-1}\right):$ reference model

$Q=\lambda\left(1-q^{-1}\right) /\left(1-0.95 q^{-1}\right)$ :generalised control weighting

with this choice, the gain of $\mathrm{H}\left(\mathrm{q}^{-1}\right)<l$ when $\lambda=7.0$ and the gain is greater than 1 when $\lambda=4.0$. Hence $\lambda=7.0$ should be sufficient for closed loop stability, despite the underestimated delay. Fig. 5-j2. shows the result of a simulation run. From time instants 1000 to $3900, \lambda=7.0$ and the controljed system is stable, ajthough there is a consjderable offset when the setpoint is non-zero. At time $=1900, \lambda$ is changed to 4.0, and the system "blows up" quickjy.

This simulation shows that wej] posedness is not just a technica] concept, but is necessary for robust stabj]jty.

This example ajso illustrates the instabjjity results of Section 3.5. When $\lambda$ is in the range 0.1 to $0.5, H\left(\mathrm{q}^{-1}\right)$ has an unstable pole and the conditional gain of $H\left(q^{-1}\right)$ is less than 1 . Simulation showed that the controljed system blew up quickly in each case.

\subsection{NONTINNFAR F.XAMPJ.F.S}

When the plant perturbations, $M_{1}$ or $M_{2}$ are nonlinear, it i.s not possible to keep the evaluation of gains for checking robustness in the frequency domain. Furthermore, pJant perturbations at the input cannot a]ways be treated in the same way as plant perturbations at the output. When the nonjinearity is memoryless, continuous]y frechet differentiabje with a graph passing through the origin and when control weighting $Q=0$, Iemma $J$ of Section 3.4.J provides considerable simplification in evaluating the gain of the $H_{0}$ or $H_{i}$ blocks in the error feedback systems of section 3.3. When $Q \neq 0$, section 4.2.1 describes a case when the 


\section{DF.STGN F.XAMPT.F.S}

evaluation of gain can be simplified. Section 4.2 also shows that $Q \neq 0$ when there is a probjem with wej]-posedness.

In Appendix $D$ some standard nonjinearjties and thejr gajns, sector bounds etc. are presented. Note that in choosing to characterjse nonjinearjties by their gains, simplicity in robustness anajysis is achjeved. With sujtabje scajing, many of the nonjinearitjes in Appendix $D$ are characterjsed by the same gain parameters. However a price for this simplicjty is not being abje to deduce the contro] performance according to other control crjteria.

In the class of invertible nonlinearjties, a pH type nonjinearity for example, occupjes a very large sector. This jmpjjes that to meet the condjtions for stabjljty, the jinear part of $H_{0}$ or $B_{i}$ in the error feedback systems of section 3.4 must have a very smajl gain. Note however that these condjtions are only sufficient. It could be very djffjcujt and not very sensjbje to design the sejf-tuner to meet these condjtions. In this case, the results of this thesis should be regarded as providing design guidelines only. AJternatively, it may be argued that it is not necessary to consjder the whole pH characterjstjc, but only a smalj region around the operating pojnt. Hence the sector bounds will be smaller and more manageabje.

In F.xample I below, an jdealised pr characterjstic is consjdered.

Fxample ] (pH Nonlinearjty) 
The system considered is of the form of Fig. 5-13 below.

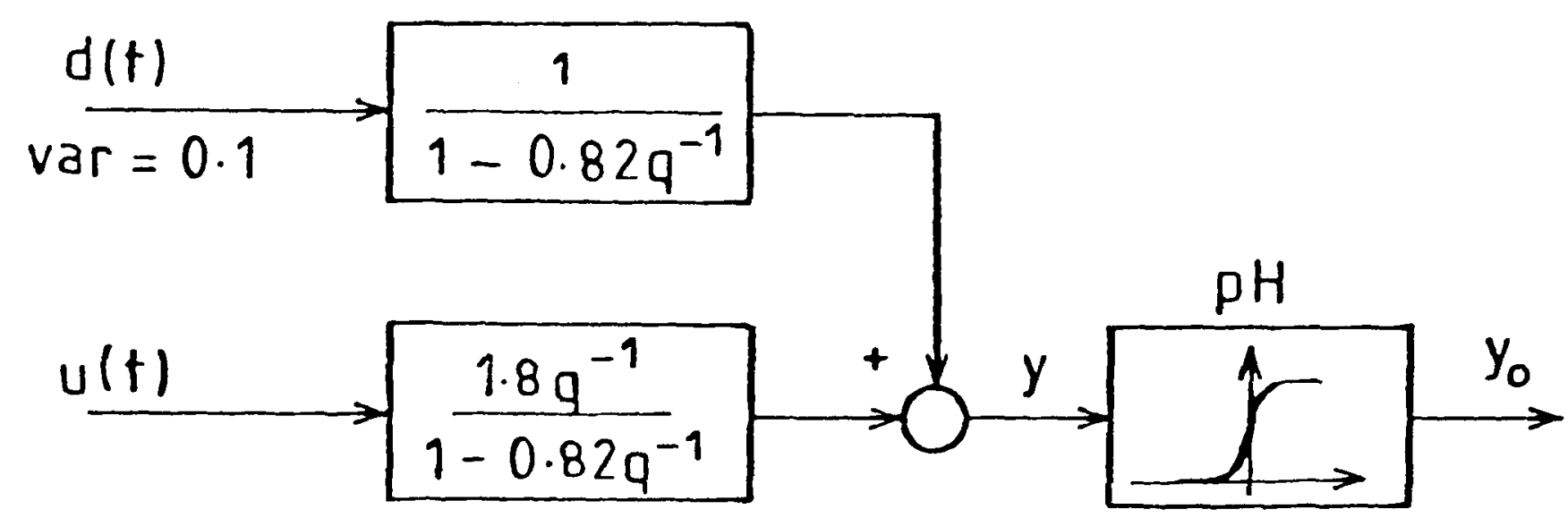

Fig. 5-13 Linear Plant with pH Nonlinearity

A first order self-tuning controller with a separate bias estimator (described in section 4.3 .1 ) is used. The setpoint is a constant at 8.5. Consider first the case when the control weighting and predictor poles are fixed as:

$$
c\left(q^{-1}\right)=1 \text { and } Q\left(q^{-1}\right)=0.1\left(1-q^{-1}\right) /\left(1-0.9 q^{-1}\right)
$$

The mode] reference polynomial is sejected as:

$$
P\left(q^{-1}\right)=\frac{1}{1-p}\left(1-p q^{-1}\right)
$$

For three choices of the desired closed loop pole, the mean and variances of the estimated prediction error, $\hat{e}_{1}$, input and output are as listed in Tabje J bejow.

As the desired pole becomes slower, note that the variances of output and $\hat{e}$, move in opposite directions. This is similar to the linear case in Fxample 1 of section 5.2. Fig. 5-14 shows a typical simulation run. The sharp dips of the output beneath the setpoint are due to the very large gain of the system in that direction. Despite the nonlinearity, the assumption of small $\sigma(t)$ still seems valid. 


\section{Table 1}

Mean and Variance as a function of $\mathbf{P}$

\begin{tabular}{|c|c|c|c|c|c|c|}
\hline \multirow[t]{2}{*}{$\mathbf{p}$} & \multicolumn{2}{|c|}{$\hat{\mathrm{e}}_{1}$} & \multicolumn{2}{|c|}{$Y$} & \multicolumn{2}{|c|}{$\mathbf{u}$} \\
\hline & mean & var & mean & var & mean & var \\
\hline 0.5 & .009 & 6.41 & 8.51 & 2.13 & .012 & sma 11 \\
\hline 0.8 & .165 & 2.5 .92 & 8.66 & 2.20 & .017 & sma 11 \\
\hline 0.95 & .291 & 366.5 & 8.79 & 1.99 & .019 & small \\
\hline
\end{tabular}

Instead of a slow model, the examples of the previous section indicated that a slow predictor could be used. Fig. 5-15 shows the result of a simulation run with a fast model but a slow predictor i.e

$$
P\left(q^{-1}\right)=1 \text { and } \quad c\left(q^{-1}\right)=1-0.9 q^{-1}
$$

$Q$ is as above. The varjance of $\hat{e}_{1}$ dropped dramatically to 1.83 , so did the variance of the output, to 2.84 . However, this was at the cost of a larger input variance.

$\mathbf{Q}$ in this example was chosen to provide some control weighting, but to have zero steady state gain. If this weighting is removed, i.e. $Q=0$, the variances of the output and prediction error are marginally smaller, without a significant increase in input variance. Hence control weighting is not important in this case. From the robust stability point of view, this is not surprising because the nonlinearity of this example meets the requirements of the special case discussed in Section 4.2.1 (if only a small sector of the nonlinearity is considered). Hence the gain of $H_{0}$ can be evaluated as in Eqn. 4-22. 


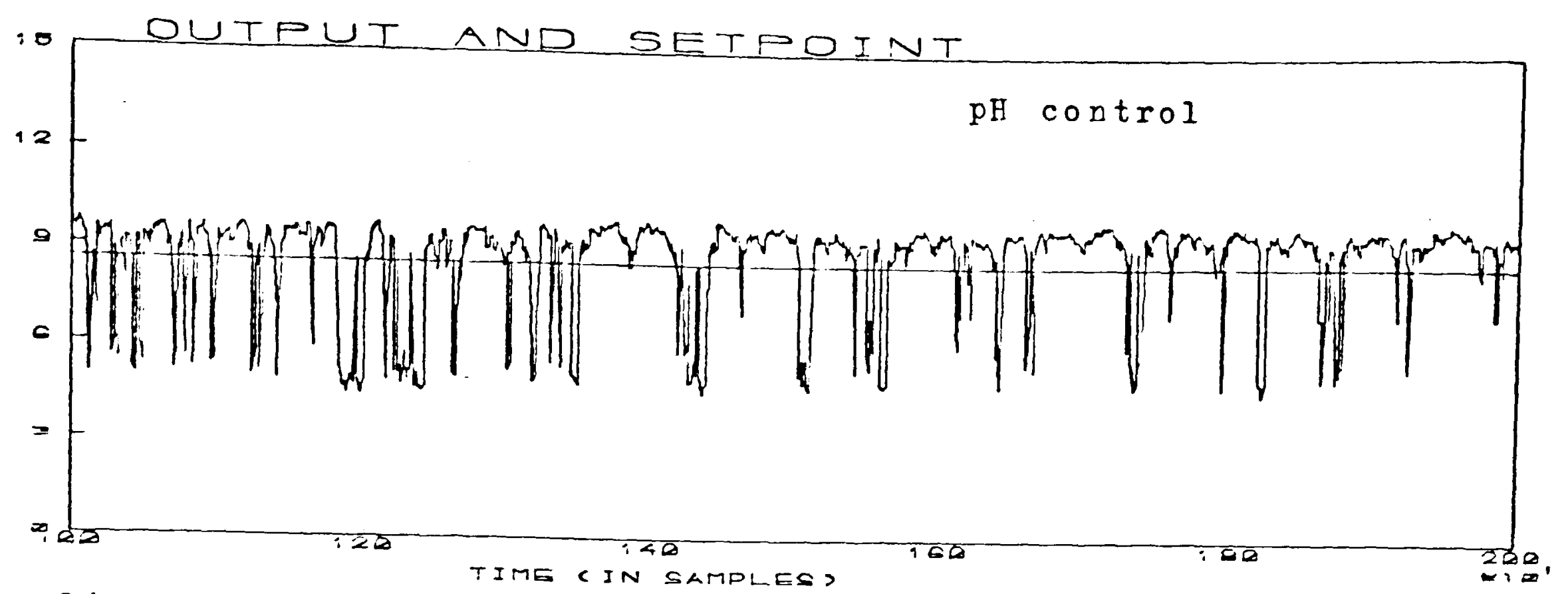

Fig. 5-14a 1st order self-tuner with separatebias estimation
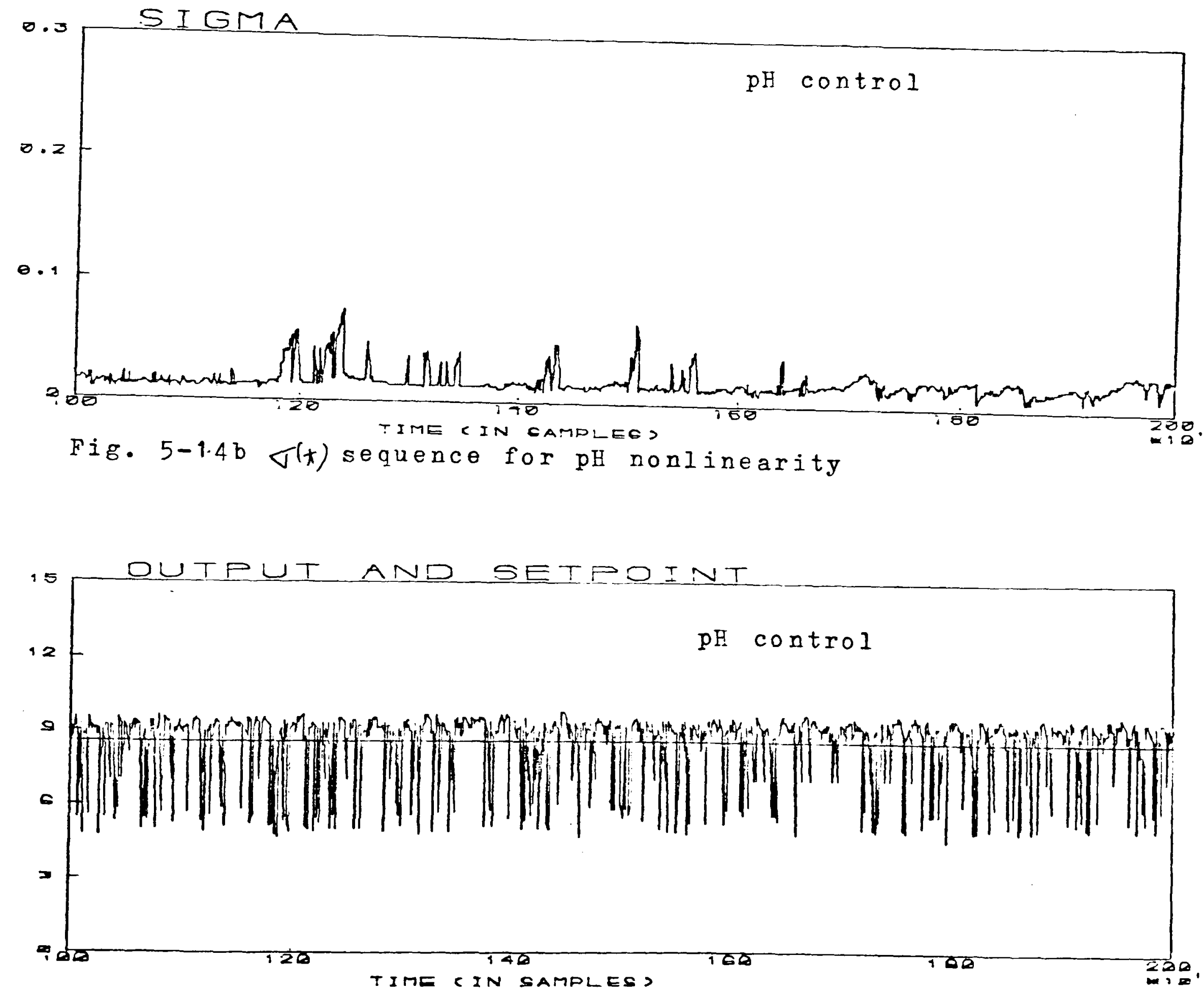

Fig. 5-15a pH control with slow predictor 
Thus far, only the design tradeoffs between predictor poles $c$ and reference model poles $p$ have been considered. The results above indicate that a slow predictor is advantageous for reducing output and prediction error variances. Another possible design tradeoff is between $P$ and the setpoint filter, $R\left(q^{-1}\right)$. F.qn. 4-2.) indicates that if $P(1)$ i.s large, the gain of $H_{0}$ i.s small and hence the system is more robustly stable. However we have also required that $P(1)=2$ for mero offsets. In principle, one way around this problem is to have a setpoint filter with $R(1)=P(1)$. Great care must be taken with this approach because, depending on the implementation, this could result in a data vector with l.arge mean levels, leading to numerical problems in the estimator. Furthermore, with nonlinearities like pH, which have severe saturation type characteristics, a larger $R(1)$ could drive the auxiliary output, $\Phi(t)$ further into saturation.

This example indicates that even though it may not be possible to satisfy the conditions for stability in this case, they still provide useful guidelines in the design. In this case, it appears that a combination of a fast model and a slow predictor performs better than a slow model and a fast predictor at the cost of more active control.

\section{Fxample 2 (Non-Tnvertible Nonlinearity)}

In Chapter 4, a system with a saturation nonljnearjty was given as an example of an $j l l$-posed system. In this example, this is jllustrated with a saturation at the input of the nominal plant. The system is shown in Fjg. 5-j6 below. 


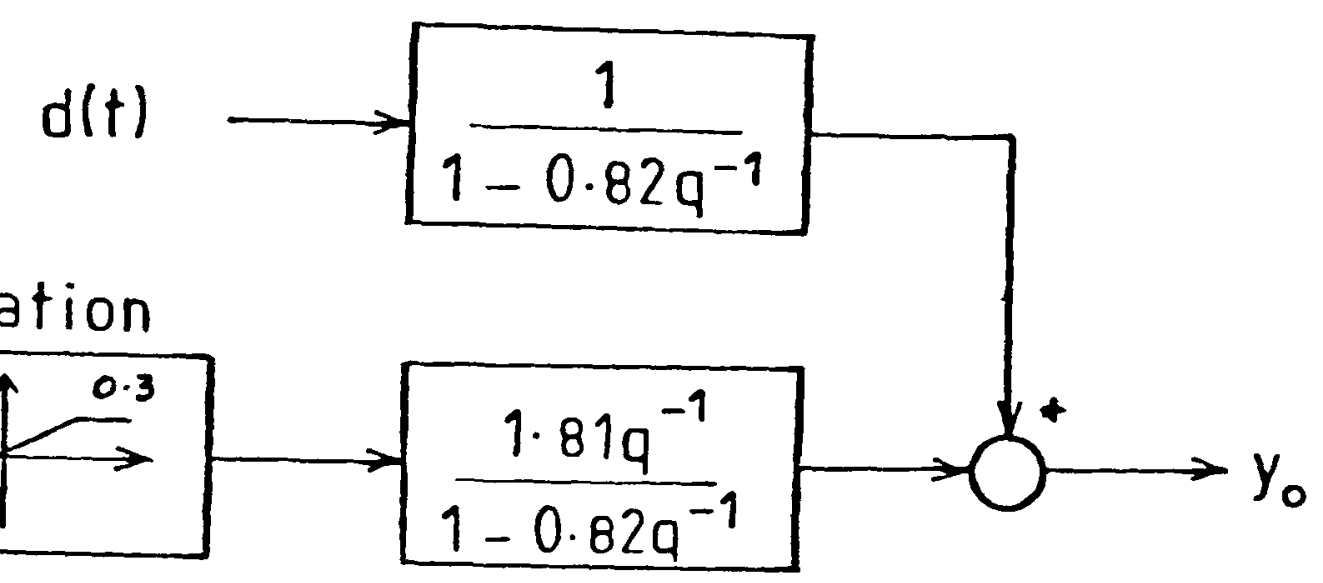

Fig. 5-16 Saturation at Input of Nominal Plant

The reference model polynomial is $P=2.5\left(1-0.6 q^{-1}\right)$. Initially, a simple scajar control wejghting, $Q=0.3$ is applied. Fjg. 5-37 shows a simulation run. This smaj] control wejghting is sufficjent to ensure stabijity. When the wejghting is removed, j.e. $Q=0$, the corresponding error feedback system is not wej]-posed. In this example, this is reflected in the inputs, which become unstable. 


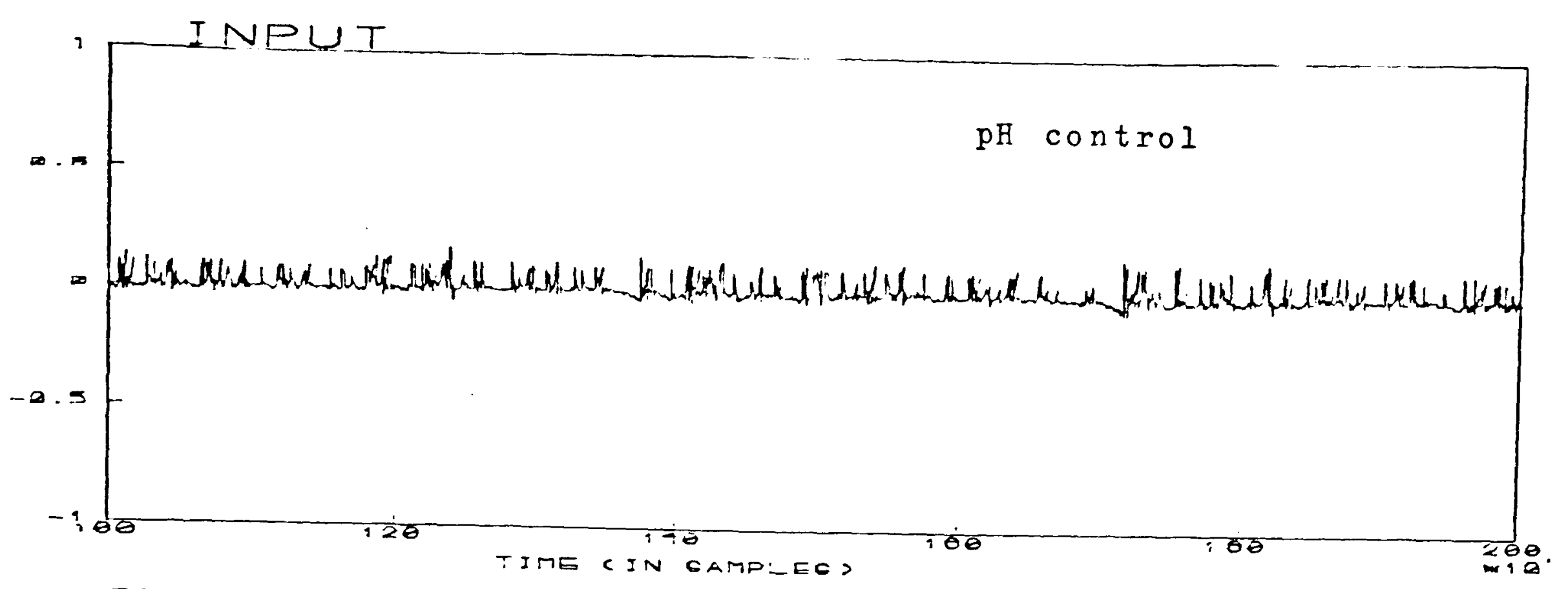

Fig. 5-15b Input from self-tuner with slow predictor

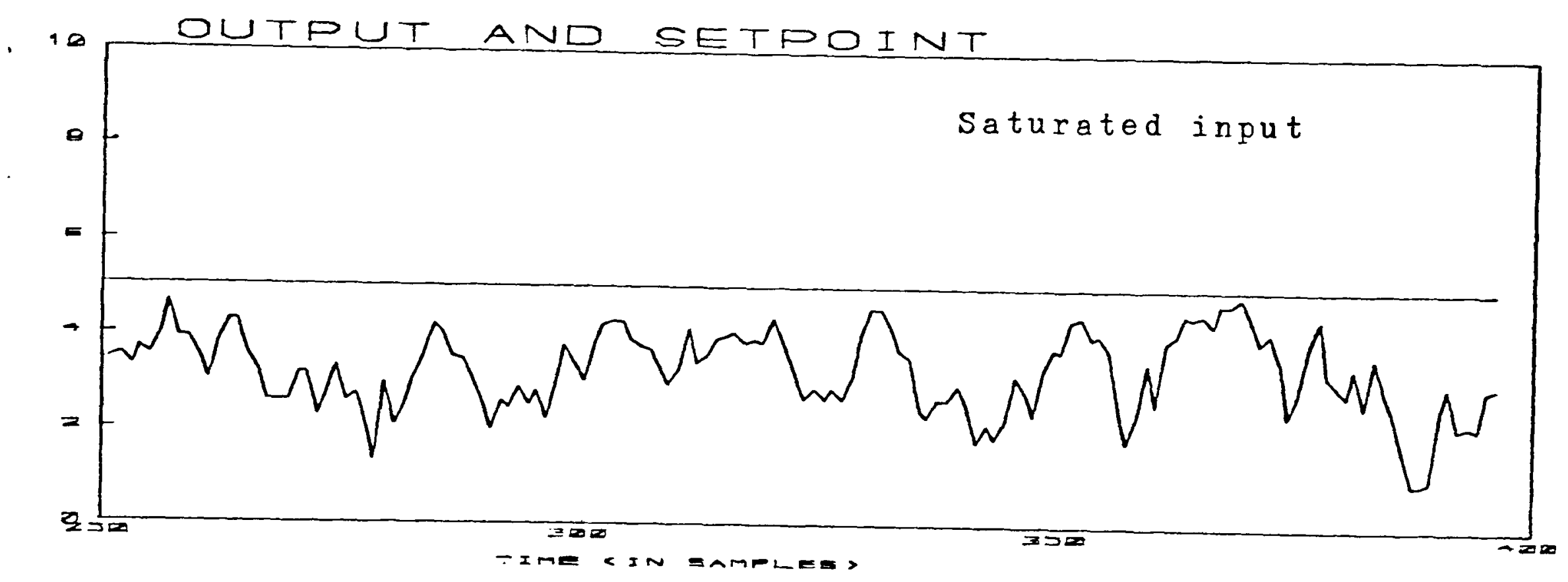

Fig. 5-17a Coping with saturation

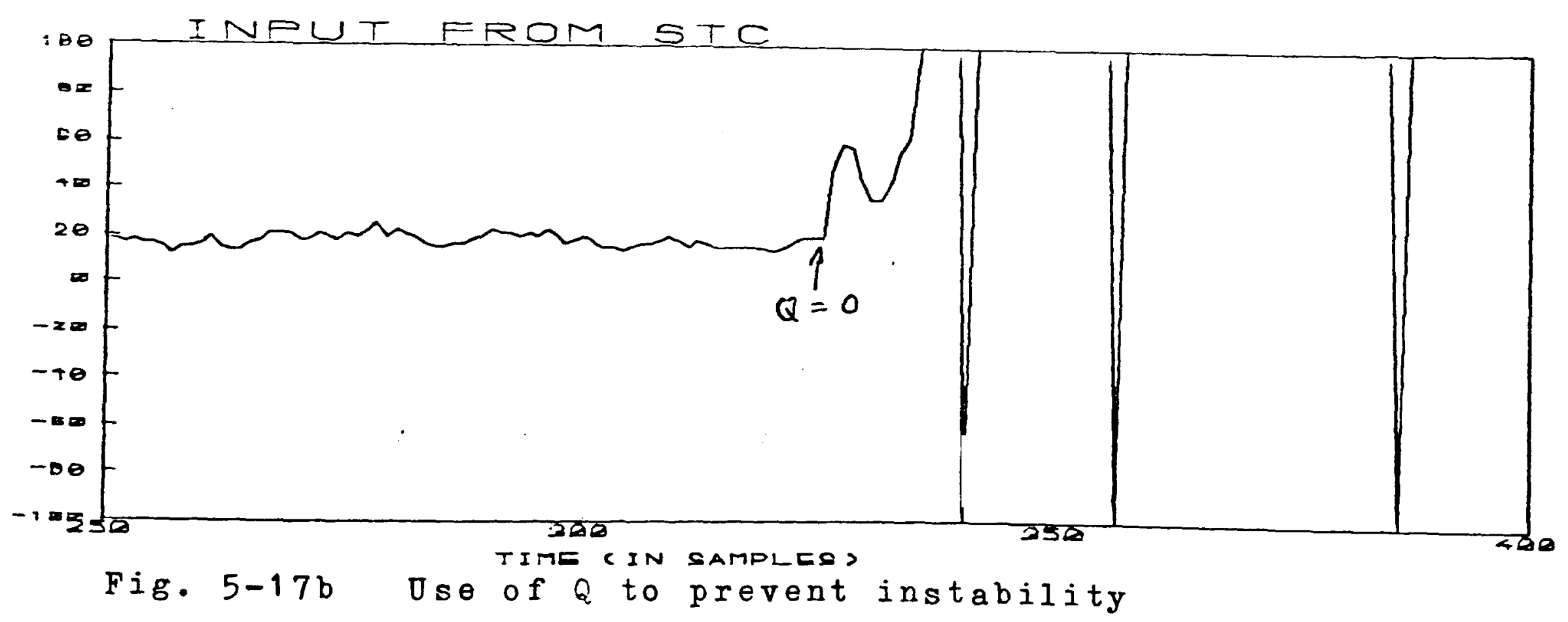




\section{CAAPTTFR 6}

CONCTIISSTONS

In this thesis, we have largely been concerned with the robust stability of the clarke-rawthrop self-tuning controller. Tt is assumed that the self-tuning controller is set up for some nominal plant or system which differs from the 'true' plant. The 'true' plant is modelled as a composition of linear dynamics (the nominal plant) and unknown linear dynamics or memoryless nonlinearities.

\subsection{NONADAPTTVF. SFT.F-TUNTNG CONTROTITF.R}

The self-tuning controller is based on a certainty equivalent control law. For this reason, the first results of this thesis are for the nonadaptive version of the self-tuning design method. It has been shown that given a nominal plant and plant uncertainty in terms of a gain measure or frequency response, it is possible to choose a priori the self-tuning parameters to ensure stability. Besides the functions indicated by their names, these parameters, the model reference polynomial, $P$, the generalised control weighting $Q$ and the fixed observer or predictor poles, c also act together as a filter on plant uncertainty. Thus there is considerable scope for design tradeoffs. If a fast model is required for instance, a slower predictor may be used to retain robust 
CONCTIISTONS

stability. Tt is also shown that as far as robust stability is concerned, there is no difference between nonlinearities at the input or at the output of the nominal plant.

When there is no plant uncertainty, the nonadaptive self-tuning controller i.s optimal in the sense that all. disturbances which can be, are rejected by a causal control law. This i.s achieved together. with setpoint following. When there is plant perturbation, it is shown that i.t is also possible to design for maximising disturbance attenuation using the same design parameters and frequency response curves as those used for designing robustness. It is also shown that when there is plant perturbation, tracking a non-mero setpoint is detrimental to disturbance rejection.

Fixplicit pole-placement type algorithms for self-tuning control. are often said to be more robust than the implicit type algorithms. Here it is shown that for the nonadaptive design method, this is not true as the $Q$ design factor can be selected to give the same stability margins.

\subsection{ADAPTTVF, SFT.F-TTNTNG CONTROT,}

The presence of an estimator in the adaptive algorithm provides an extra degree of freedom in the controller design. It is shown that the estimator can be represented by a nonlinear time-varying operator which is dissipative and has a finite $I_{2}$ gain. The estimator relationship is also shown to be uniformly bounded if a non-unity forgetting factor is used. 


\section{CONCTIISTONS}

When there is plant perturbation, the closed loop system with the implicit self-tuning controller is shown to be not only $l_{2}$ stable but a.lso $3_{\infty}$ stable as well. Section 4.2 shows that these results are valid for all integer time delays. When the noise parameters are not estimated, stability i.s relatively trivial. but when they are estimated, stability depends on the noise parameters satisfying a common positive rea]. condition.

When there is plant perturbation, the presence of a nonlinear time-varying relationship like the estimator makes analysis more difficult than in the nonadaptive case. However, the extra degree of freedom given by the estimator means that for robust stability, it is only required that there exists some nominal plant which meets the requirements for robustness. As in the nonadaptive case, the conditions for robust stability are also in terms of the self-tuner design parameters. Toosely stated: adaptation here means that if there exists a robust 'state', the self-tuner will find it. The results are in terms of both $\mathrm{T}_{2}$ and $\mathrm{l}_{\infty}$ stability. An important feature is that the latter. follows almost trivially from the former if a non-unity forgetting factor is used.

When the plant perturbations is linear, the robust stability results are also complemented by instability results.

When an explicit type self-tuning algorithm is used, section 3.6 has shown why it is difficult to get stability, let alone robustness results. However for the simple case where we assume that the nominal plant has an auto-regressive type model, it is possible to get robustness results. These indicate that the explicit self-tuner is not robust to non-minimum 


\section{CONCTIUSTONS}

phase 7.eros.

The gain of the estimator used in the preceding analysis depends on an a priori. unknown quantity $\sigma_{0}$. Tt is shown in section 3.7 that this scalar has nice properties if the inputs and outputs of the system are "persistently exciting" in a well-defined sense. In a feedback control system though, it is not easy to know a priori. whether the data i.s "persistently exciting". Hence for the purpose of design, we assume that $\sigma_{0}$ is smal.1. simulation examples indicate that this is a valid assumption.

One way around the above problem is to consider an alternative estimator. This estimator (the scalar gain estimator) is shown to give nice theoretical results, but is less robust (and less useful in practice too) than the matrix gain estimator.

Most of the above mentioned results were for the case of minimal delay $(k=1)$. For the general delay case, the multiple recursion algorithm of Goodwin et al i.s shown to work. However, the ordinary matrix gain estimator is also shown to work for the general delay case, at the cost of reduced stability margins.

Although the $Q$ design polynomial/transfer function has hitherto been regarded as a generalised form of control weighting, it is shown in this thesis that i.t has a role too in robustnesss by ensuring the realisability of the feedback system.

The basic self-tuning controller is designed to cope with disturbances which have zero means. When this is not true, various additions to the basic algorithm are needed. In this thesis, we have 
CONCTIUSTONS

considered some of the common ones and it has been shown that the robustness of these composite algorithms can be studied using the same methods as those employed here.

Finally some simulation examples have been included to illustrate the robustness results. These show some of the tradeoffs which have to be made between robustness, model following, disturbance rejection and tracking. When there are plant perturbations, the self-tuning controller does not have sufficient degrees of freedom to satisfy all these design criteria simultaneously.

\subsection{SOMF. FURTHFR RF.SF.ARCH ARFAS}

Robust stability is a relatively recent subject of research in control theory. In the self-tuning control literature, there is very little systematic discussion of the subject. The results presented in this thesis are focussed on one type of self-tuning algorithm: the clarke-Gawthrop self-tuning controller. For an important alternative, the explicit algorithms, only preliminary results were obtained. Hence there is scope for further research on robustness, not only of explicit but also for other varieties of self-tuning controllers such as hybrid algorithms and those based on state-space models.

There has also been fresh interest in multivariable self-tuners and with the current surge of interest in the robustness of multivariable systems, the opportunity exists for new multivariable self-tuning designs which are robust. 


\section{REFERENCES}

[ 1] Allidina, A.Y. and Hughes, F.M. (1980) Generalised self-tuning controller with pole assignment Proc. IEE, Vol. 127, Pt. D, No. 1 .

[ 2] Allidina, A.Y.,Bughes, F.M., and Tye,C. (1981) self Tuning Control for systems employing feedforward Proc. IEE, Vol. 128, Pt. D, No.6

[ 3] Anderson, B.D.O. (1972)

External and Internal stability of Linear systems: a new connection IEEE Trans, Vol. AC-17 No.1, Feb. 1972

[ 4] Anderson, B.D.O., and Johnson, C.R. (1981)

Exponential Convergence of Adaptive Identification and control Algorithms

IFAC World Congress, 1981, Kyoto: also in Automatica

[ 5] Anderson, B.D.O., and Moore,J.B. (1971)

Linear Optimal Control

Prentice Hall

[ 6] Astrom, K.J. (1970)

Introduction to stochastic control theory

Academic Press

[7] Astrom, K.J. (1980)

Robustness of a design method based on assignment of poles and zeros IEEE Trans, Vol. AC-25 No.3, June 1980 pp.588-591

[ 8] Astrom, K.J. and Wittenmark, B. (1973)

On self-tuning regulators

Automatica, Vol. 9 No.2, March 1973, pp. 185-199.

[ 9] Astrom, K.J., Borisson, U., Ljung, L. and Wittenmark, B. (1977) Theory and applications of self-tuning regulators Automatica, Vol. 13 No.5, sept 77, pp. 457-476.

[10] Astrom, K.J., Westerberg, B., and Wittenmark, B. (1978)

Self-tuning controllers based on pole-placement design

Report LUTFD2/(TFRT-3148)/1-052/(1978), Lund Institute of Technology 
[11] Astrom, K.J., and Wittenmark, B. (1980)

Self-tuning controllers based on pole-zero placement

Proc. IEE, Vol. 127, Pt.D, No.3, May 1980 pp.120-130

[12] Billings, s., and Fakhouri (1979)

Identification of systems composed of linear dynamics and static nonlinear elements

IFAC Symposium on Identification, Darmstadt

[13] Billings, S.A., and Fakhouri,S.Y. (1978)

Theory of separable processes with applications to the identification of nonlinear systems

Proc. IEE, Vol. 125 No.9, Oct. 1978 pp 1051-1058

[14] Billings, S.A., and Leontaritis, I.J. (1981) Identification of Nonlinear systems using parameter estimation
techniques

Proc. IEE Conf. on Control and its Applications, Warwick, Mar 81

[15] Bitmead,R,R., and Anderson,B.D.O. (1980)

Performance of adaptive estimation algorithms in dependent random environments

'Mini and Microcomputers in Control', San Diego, 1979; also in IEEE Trans. AC Aug. 80

[16] Box, and Jenkins (1975)

Time Series Analysis: Forecasting and Control

Holden Day (Revised Edition)

[17] Brockett,R.W., Lee, H.B. (1967)

Prequency Domain Instability Criteria for Time Varying and Nonlinear Systems

Proc. IEEE, Vol.55 No.5, May 1967

[18] Clarke, D.W. (1981)

Model Following and pole placement self-Tuners

presented at Budapest conference

[19] Clarke, D.W. and Gawthrop, P.J. (1975)

Self-tuning controller

Proc. IEE, Vol. 122 No. 9, pp. 929-934.

[20] Clarke, D.W. and Gawthrop, P.J. (1979)

Self-tuning control

Proc. IEE, Vol. 126, No. 6, June 79 pp.633-640.

[21] Clarke,D.W. (1981)

Implementation of self tuning controllers

Chap 6 of 'Self-Tuning and Adaptive Control', IEE Control Series 15

[22] Clarke,D.W. (2981)

Introduction to self Tuning controllers

Chap 2 of 'Self-Tuning and Adaptive Control', IEE Control series 15 
[23] Cordero,A.O., Mayne, D.Q. (1981)

Deterministic convergence of a self-tuning regulator with variable forgetting factor

Proc. IEE, Vol. 128 Pt.D, No.1, Jan.1981, Pp.19-23

[24] Cruz,J.B.,Freudenberg,J.S., and Looze,D.P. (1981)

A Relationship between sensitivity and stability of multivariable feedback systems

IEEE Trans, Vol. AC-26 No.1, Feb 1981

[25] Cruz,J.B.,Looze, D.P., and Perkins,W.R. (1981)

Sensitivity Analysis of Nonlinear Feedback Shystems

J.Franklin Institute, Sept/October 81

[26] De Larminat, P. (1979)

On overall stability of certain adaptive control systems

Proceedings of the IFAC Symposium on 'Identification and system Parameter Estimation', Darmstadt, FRG.

[27] De Larminat, P. (1980)

An unconditional stabiliser for non-minimum phase process

International symposium on adaptive systems, Bochum, FRG.

[28] Desoer, and Vidyasagar (1975)

Feedback Systems:input-output properties

Academic Press

[29] Desoer,C.A. (1978)

Perturbation in the I/O Map of a Non-linear Feedback system caused by large plant perturbation

J.Franklin Institute, sept. 78

[30] Desoer, C.A., and wang (1979)

Robust Nonlinear servomechanism problem

Int. J. Cont., Vol. 29, No.5, May 79

[31] Doob, J.L. (1953)

Stochastic processes

wiley

[32] Doyle,J.C., and Stein,G. (1977)

Robustness with Observers

IEEE Trans, Vol. AC-24 No.4

[33] Egardt, B. (1979)

Stability of Adaptive Controllers

Springer Verlag lecture notes on Control and Information sciences, No. 20

[34] Fortescue, T.R., Kershenbaum, L.S., and Yastie, B.E. (1979) Implementation of self-tuning regulators with variable forgetting factors

Report, Department of Chemical Engineering and Chemical Technology, Imperial College 
[35] Franklin, G.F., and Powell, J.D. (1980) Digital control of dynamic systems Addison wesley

[36] Fuchs, J.J.J. (1980) Explicit Self-Tuning Methods

Proc. IEE, Vol. 127 Pt..D, No.6, Nov.80 pp259-264

[37] Gawthrop, P.J. (1977)

Some interpretations of the self-tuning controller

Proc. IEE, Vol. 124 No. 10 , oct 77 pp. 889-894.

[38] Gawthrop, P.J. (1978)

On the stability and convergence of self-tuning algorithms

Oxford University Engineering Lab. Report No. 1259/78

[39] Gawthrop, P.J. (1980)

Bybrid self-tuning control

Proc. IEE, Vol. $127 \mathrm{pt}$. D No. 5, Sept 80 pp. 229-236.

[40] Gawthrop, P.J. (1980)

On the stability and convergence of a self-tuning controller

Int. J. Control, vol. 31 No.5, March 80, pp. 973-998.

[41] Gawthrop, P.J. (1981)

Some properties of discrete adaptive controllers

Chap 5 of 'Self-Tuning and Adaptive Control', IEE Control series 15

[42] Gawthrop, P.J. and Lim,K.w. (1982)

Robustness of self-tuning controllers

Proc. IEE, Vol. 129 Pt.D No.l, Jan 1982

[43] Goodwin, G.C., Ramadge, P.J. and Caines, P.E. (1979)

Ultimate objectives and prior knowledge in system identification

Proc. of the IFAC Symposium on Identification and System Parameter Estimation', Darmstadt, FRG.

[44] Goodwin, G.C., Ramadge, P.J., and Caines, P.E. (1980)

Discrete-time multivariable adaptive control

IEEE Trans, Vol. AC-25 No.3, June 80 pp.449-455

[45] Goodwin, G.C., and Ramage, P.J. (1979)

Design of restricted complexity adaptive regulators

IEEE Trans, Vol. AC-24 No. 4, Aug 79, pp.584-588

[46] Goodwin, G.C., and Sin, K.S. (1979)

Adaptive control of non-minimum phase systems

The University of Newcastle, NSW, Australia, Report EE 7918

[47] Goodwin, G. and Caines, P.E. (1981)

Discrete time stochastic adaptive control

Siam Journal of Control, vol. 19 No. 6 
[48] Goodwin, G.C., and sin,K.s. (1981)

Adaptive control of nonminimum phase systems

IEEE Trans, Vol. AC-26 No.2, April 81, Pp478-482

[49] Goodwin,G.C.,Kwai,S.S.,Saluja,K.K. (1980)

Stochastic Adaptive Control and Prediction-The General
Delay-Coloured Noise Case

IEEE Trans, Vol.AC-25 No.5, October 80

[50] Horowitz (1963)

Synthesis of Feedback systems

Academic press

[51] Kalman, R.E. (1958)

Design of a self-optimising control system

Trans. ASME, Feb 1958, pp. 468-478

[52] Kalman, R.E. (1964)

When is a linear control system optimal ?

J. Basic Engineering, Vol. 86, pp. 51-60

[53] Kreisselmeier,G. (1982)

On Adaptive State Regulation

IEEE Trans, Vol. AC-27 No.1, Feb 82

[54] Kucera, v. (1979)

Discrete linear control

wiley

[55] Kuo, Benjamin (1980)

Digital Control Systems

Holt, Rinehart and Winston Inc.

[56] Kwakernaak, H. and Sivan, R. (1972)

Linear optimal control systems

wiley

[57] Lam, K.P. (1980)

Implicit and explicit self-tuning controllers

D.Phil Thesis, oxford University

[58] Landau (1979)

Adaptive Control: the model reference approach

Marcel Dekker

[59] Landau, I.D. (1974)

A survey of model-reference adaptive techniques - theory and applications

Automatica, Vol. 10 No. 4, July 74, pp 353-379.

[60] Lawrence, P.J. (1981)

Estimation of the Volterra functional series of a nonlinear system using frequency response data

Proc. IEE Pt. D, vol. 128 No.5, Sept. 81 pp 207 
[61] Leitch,R.R. (1982)

Input output relative stability measures

ProC. IEE Pt. D, Vol.129 No.1, Jan. 82

[62] Lin, Y-B., and Narendra, K.S. (1980)

A new error model for adaptive systems

IEEE Trans, Vol. AC-25 No.3, pp.585-587

[63] Ljung, I. (1977)

Analysis of recursive stochastic algorithms

IEEE Trans, Vol. AC-22 No. 4, Aug 77, pp 551-575.

[64] Ljung, I. (1977)

On positive real transfer functions and the convergence of some recursive schemes

IEEE Trans, Vol. AC-22 No. 4, Aug 77, pp 539-550

[65] Ljung, I. and wittenmark, B. (1974)

Asymptotic properties of self-tuning regulators

Report 7404, Lund Institute of Technology

[66] Ljung,L. (1981)

The ODE approach to the analysis of adaptive control systems-possibilities and limitations

Proc. JACC, 1981

[67] Moden,P.E. (1981)

Stochastic Control with a sliding short time criterion and offset elimination

Report, Uptec 8149R, April 1981, Insti. of Tech., Uppsala University

[68] Molander, P. (1979)

Stabilization of uncertain systems

Report LUTFD2/(TFRT-1020)/1-111/(1979), Department of Automatic Control, Lund Institute of Technology

[69] Morris, A.J., and Nazer, Y. (1978)

Self-tuning controllers for single and multivariable systems.(in two parts)

Internal Report, University of Newcastle

[70] Narendra, K.S., and Lin, Y-B. (1980)

Stable discrete adaptive control

IEEE Trans, Vol. AC-25, No.3, June 80, pp.456-461

[71] Ogata, K. (1970)

Modern Control Engineering

Prentice Hall Electrical Engineering series

[72] Peterka, v. (1970)

Adaptive digital regulation of noisy systems

Proceedings of the IFAC symposium on 'Identification and Process Parameter Estimation', Prague 
[73] Peterka, V. (1975)

A square Root filter for real-time multivariable regression Kybernetika, Vol. II No. 1, pp 53-67

[74] Peterka,v. (1981)

Predictor based self tuning control

Presented at Oxford Internal seminar

[75] Samson, C., and Fuchs, J-J (1981) Discrete adaptive regulation of not-necessarily minimum-phase
systems

Proc. IEE Pt. D, Vol. 128 No.3, May 1981, pp 102-108

[76] Sandberg, I.W. (1965)

Some results on the theory of physical systems governed by nonlinear functional equations

Bell syst. Tech. J, Vol. 44, May-June 2965

[77] Sandell,N.R. (1979)

Robust stability of systems with Application to singular

Automatica Vol. 15, pp 467-470

[78] Solo, V (1980)

Some aspects of Recursive Parameter Estimation

Int. J. Control, Vol.32 No.3, pp 395-410

[79] Solo,v. (1979)

The Convergence of AML

IEEE Trans, Vol. AC-24 No.6, Dec 79

[80] Takeda,S., and Bergen,A.R. (1973)

Instability of Feedback Systems by Orthogonal Decomposition of L2

IEEE Trans, Vol AC-18 No.6, Dec.73

[81] Thornton, C.I., and Bierman, G.J. (1978)

Filtering and error analysis via the UDU covariance factorisation

IEEE Trans, Vol.AC-23 No. 5, Oct 78, pp.901-907

[82] Titchmarsh, E.C. (1932)

The Theory of Functions

Oxford University Press

[83] Vidyasagar, M. (1981)

Input Output Analysis of Large Scale Interconnected systems Springer-Verlag series: Lecture notes in control and information Sciences

[84] Warwick, K. (2981)

Self-Tuning Regulators - a state space approach

Int. J. Control, Vol.33 No.5, May 1981, pp 839-858

[85] Wellstead, P.E., Prager, D., and Zanker, P. (1979)

Pole assignment self-tuning regulator

Proc. IEE, Vol.126 No.8, Aug 79, pp.781-787 
[86] Wellstead, P.E., Edmunds, J.M., Prager, D., and Zanker, P. (1979) Self-tuning pole/zero assignment regulators

Int. J. Control, Vol.30 No. 1 , July 79, pp 1-26.

[87] Wellstead, P.E., and Zanker, P. (1979)

Servo self-tuners

Int. J. Control, Vol.30 No.1, July 79, Pp.27-36

[88] Willems, J.C. (1971)

The analysis of feedback systems

Research monograph No. 62, MIT press.

[89] Willems, J.C. (1976)

Mechanisms for stability and instability in feedback systems

Proc. IEEE, Vol. 64 No. 1, pp 24-35.

[90] Willems, J.C. (1971)

The generation of Lyapunov functions for input-output stable systems Siam J. Control, Vol. 9, Feb 71, pp 105-134

[91] Zames (1966)

The Input output stability of time varying nonlinear feedback systems pt. 1 and 2

IEEE Trans, Vol. $A C-11$ No. 2 and 3

[92] Zames, G. (1963)

Functional Analysis Applied to Nonlinear Feedback

IEEE Trans, Vol. CT-10 No. 3, Sept. 1963

[93] Zames, G. (1964)

Realizability Conditions for Nonlinear Feedback systems

IEEE Trans, Vol.CT-11, June 64, pp 186-194

[94] Zames,G. (1965)

Nonlinear time-varying feedback systems - conditions for $I_{\infty}$ boundedness derived using conic operators on exponentially weighted spaces

Proc. 3rd Allerton Conference, October 1965

[95] Zames, G. (1981)

Feedback and Optimal Sensitivity:Model Reference Transformations, Multiplicative Seminorms and Approximate Inverses IEEE Trans, Vol.AC-26 No.2, April 81, pp 301

[96] Hodgson, A.J., and Clarke,D.W. (1982)

Self tuning applied to batch reactors

Presented at IEEE meeting on Adaptive Control, Hull, July 82 


\section{APPENDIX A}

\section{A.1 SECTION 2.2: PROOF OF IEMMA 1}

Consider Figs. A-1 to A-4 below, all of which represent the same plant, where $\mathrm{H}$ represents a linear nominal plant operator and the plant perturbations may be at the input, output or are additive.

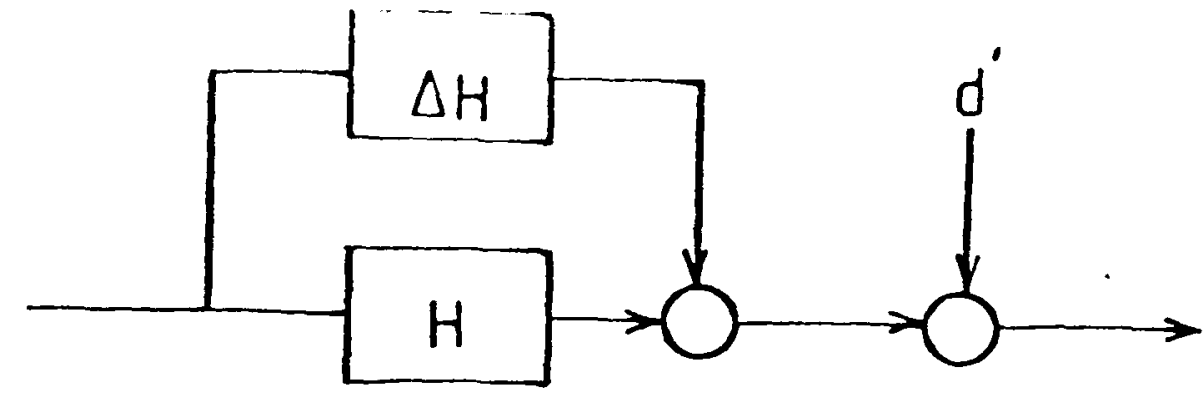

Fig. A-1
Additive PIant

Perturbation

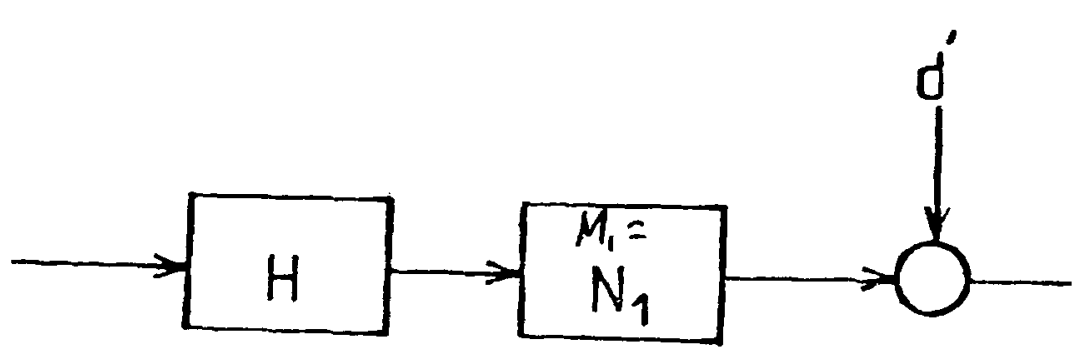

Fig. A-3 output Plant Perturbation

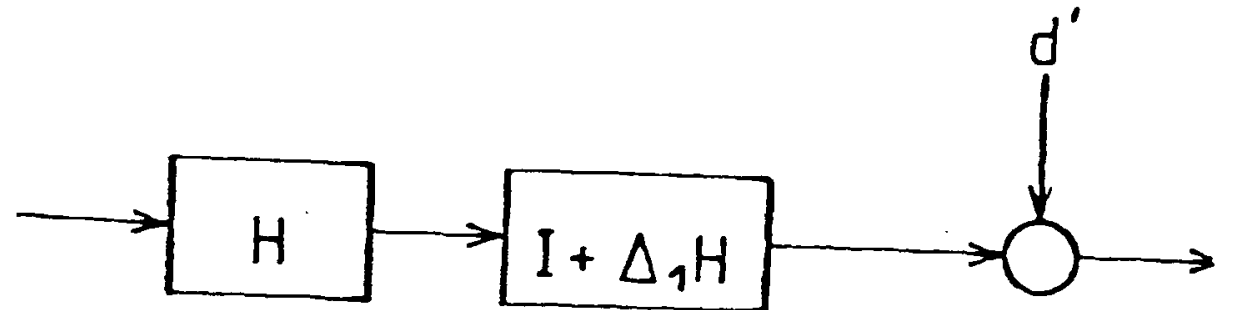

'ig. A-2 Multiplicative Plant Perturbation

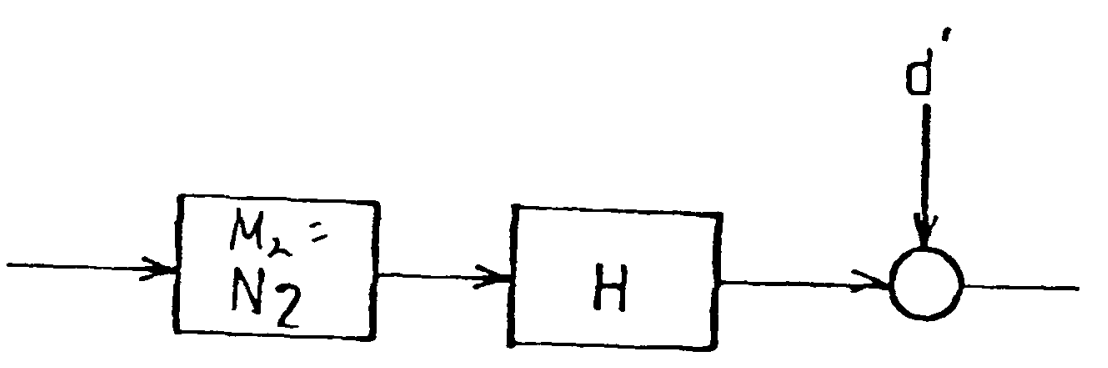

Fig. A-4 Input Plant Perturbation

From Figs. $\mathrm{A}-1$ and $\mathrm{A}-2$,

$$
\mathrm{H}+\Delta \mathrm{H}=\left[\mathrm{I}+\Delta_{1} \mathrm{H}\right] \mathrm{H}=\mathrm{H}+\left[\Delta_{1} \mathrm{~B}\right] \mathrm{B}
$$


Appendix A

Hence $\Delta \mathrm{H}=\left[\Delta_{1} \mathrm{~B}\right] \mathrm{H}$

From Figs. $A-2$ and $A-3$,

$$
N_{1}=I+\Delta_{1} H
$$

From Eqns. $I$ and $2, \Delta H=\left(N_{1}-1\right) H$

From Figs. $A-3$ and $A-4, \mathrm{HN}_{2}=\mathrm{N}_{1} \mathrm{~B}$

hence $\mathrm{HN}_{2} \mathrm{~B}^{-1}=\mathrm{N}_{1}$

From Eqns. 3 and $4, \Delta \mathrm{H}=\left[\mathrm{HN}_{2} \mathrm{~B}^{-1}-1\right] \mathrm{H}=\mathrm{HN}_{2}-\mathrm{H}$

$$
=H\left(N_{2}-I\right)
$$

A.2 SECTION 2.3.2: DERIVATION OF EQNS. 2-34 TO 2-37

From Fig. 2-2,

$$
A y(t)=B u(t-k)+C d(t)
$$

Substituting Eqn. 2-29 and multiplying by $Q$

$$
Q A Y(t)=B Q u_{0}(t-k)-B Q Q(t-k)+\operatorname{cQd}(t)
$$

substituting the control law, Eqn. 2-26

$$
\operatorname{QAy}(t)=B R W(t-k)-B \hat{\Phi}_{k}(t / t-k)-\operatorname{QBd}(t-k)+\operatorname{cQd}(t)
$$

But by definition

$$
\hat{e}_{k}(t)=\Phi(t)-\hat{\Phi}_{k}(t / t-k)=P Y_{0}(t)-\hat{\Phi}_{k}(t / t-k)
$$

jubstituting Eqn. 2-28

$$
\hat{B e}_{k}(t)=P B(Y(t)+Y(t))-B \hat{\Phi}_{k}(t / t-k)
$$

Ind

$$
\operatorname{PBY}(t)=\hat{B E}_{k}(t)-\operatorname{PB} Y(t)+B \hat{\Phi}_{k}(t / t-k)
$$

ldding Eqns. 7 and 8 ,

$$
[P B+Q A] Y(t)=B\left[\hat{e}_{k}(t)+R w(t-k)-P Q(t)-Q \bar{u}(t-k)+\frac{C Q}{B} d(t)\right]
$$

his is Eqn. 2-34. Eqn. 2-36 can be obtained by substituting Eqn. 9 in qn. 6 . 


\section{Appendix A}

\section{A.3 SECTION 2.4.1: PROOF OF ILEMMA 1}

From the control law, $\frac{F}{C} Y(t)+\left(\frac{G}{C}+Q\right) u(t)=w(t)$

and the nominal system equation, $A y(t)=B u(t-k)+d(t)$

the loop transfer function of the nominal sygtem is

$$
G_{1}=\frac{q^{-k} F B}{A(E B+Q C)}=\left[G_{2}-1\right]^{-1}
$$

where

$$
G_{2}=\frac{C(P B+Q A)}{q^{k} F B}
$$

From condition (a) of Theorem 1 (Section 2.4.1),

$$
\inf _{|q|=1}\left|G_{2}\left(q^{-1}\right)\right|>0
$$

This implies that the Nyquist plot of $G_{2}\left(q^{-1}\right)-1$ lies outside a circle of centre -1 , radius 0 . Since $C(P B+Q A)$ is always stable, and assuming that the nominal closed loop system is stable

$$
\left|G_{2}\left(q^{-1}\right)-1\right| \geqslant 1 \text { when } 4\left(G_{2}\left(q^{-1}\right)-1\right)=180^{\circ}
$$

First consider $0^{2}<2$. Construct circles centre $(0,0)$, radius 1 and centre $(-1,0)$, radius 6 in the complex plane (Fig. A-5 below)

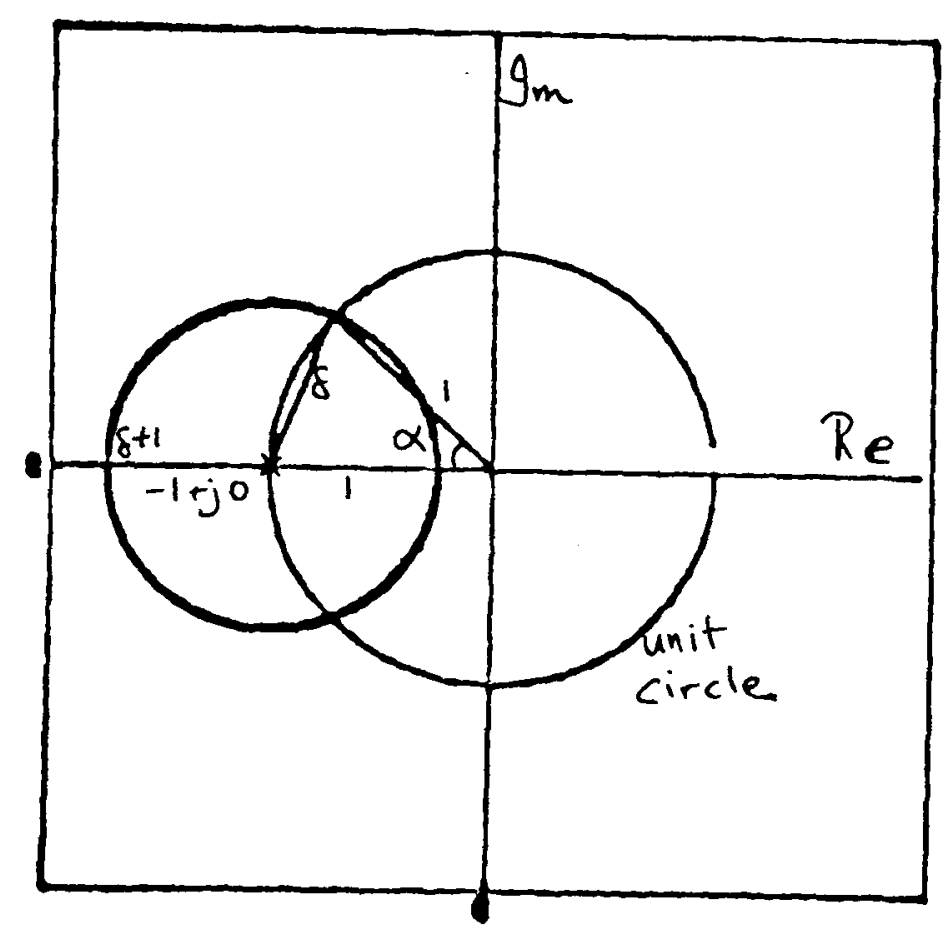

Fig. A-5 Gain and Phase Margins

$G_{2}\left(q^{-1}\right)-1$ is the inverse of the loop transfer function. Bence the Nyquist plot of $G_{2}\left(q^{-1}\right)-1$ is the inverse Nyquist plot of $G_{1}$ The minimum gain margin follows from Eqn. 10 by definition, as when the phase 


\section{Appendix A}

angle is $-180^{\circ}$, the inverse Nyquist plot is always to the left of the circle radius $0 . \alpha$ is the minimum phase margin. Applying the cosine rule of elementary trigonometry, the result follows. For $0^{2}>2$ the Nyquist plot of $\mathbf{G}_{1}$ is always in the right half plane and outside the unit circle.

A.4 SECTION 2.4.2: PROOF OF THEOREM 1

$$
9=0
$$

From Eqn. 2-32

$$
\begin{aligned}
e_{m}(t) & =q-\frac{k B E}{c} a(t) \\
= & \frac{B E}{C}\left(I-M_{2}\right) u_{0}(t-k) \\
\text { since } \hat{e}_{k}(t) & \equiv \Phi(t)-\hat{\Phi}_{k}(t) \\
& =\frac{E A}{C} y(t)-\frac{G}{C} u_{0}(t-k) \\
y(t)=\frac{C}{E A} & \hat{e}_{k}(t)+\frac{G}{E A} u_{0}(t-k)
\end{aligned}
$$

From the control law,

$$
\frac{F}{C} Y(t-k)+\frac{G}{C} u_{0}(t-k)+Q u_{0}(t-k)=R w(t-k)
$$

Substituting Eqn. 15 in Eqn. 16 we get

$$
u_{0}(t-k)=\frac{A}{P B+Q A}\left[R w(t-k)-\frac{F}{E A} \hat{e}_{k}(t-k)\right]
$$

From Eqn. 2-40

$$
\hat{e}_{1}(t)=\hat{e}_{k}(t)=e(t)-e_{m}(t)
$$

and from Eqns. 13, 17, 18 we get the feedback system of Fig. A-6 below. This reduces to Fig. A-7. Applying the small gain theorem or the Nyquist stability theorem, the result follows. 


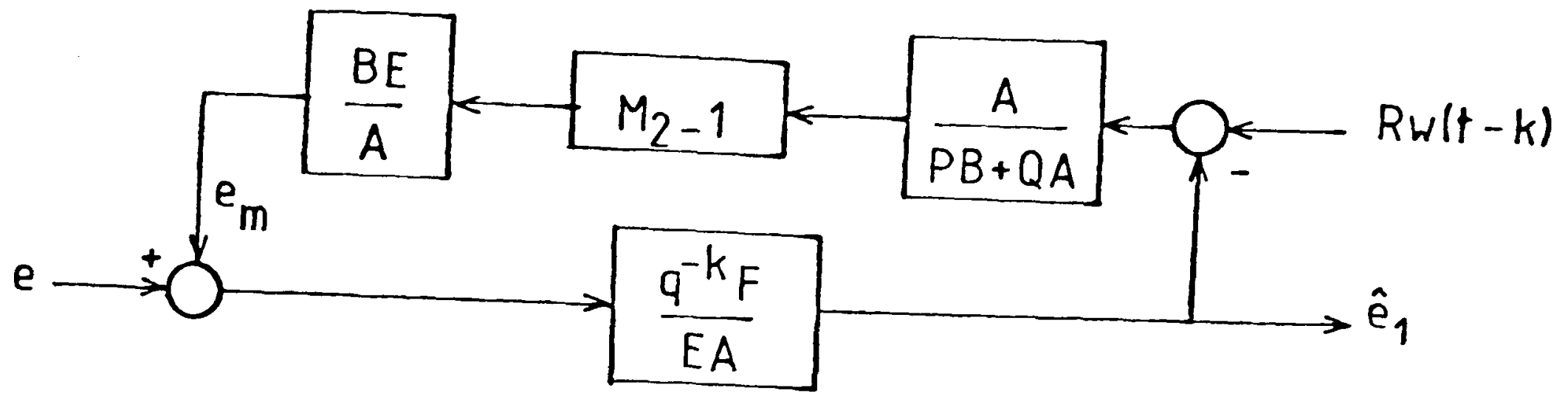

Fig. A-6 Error Feedback System for Input Error

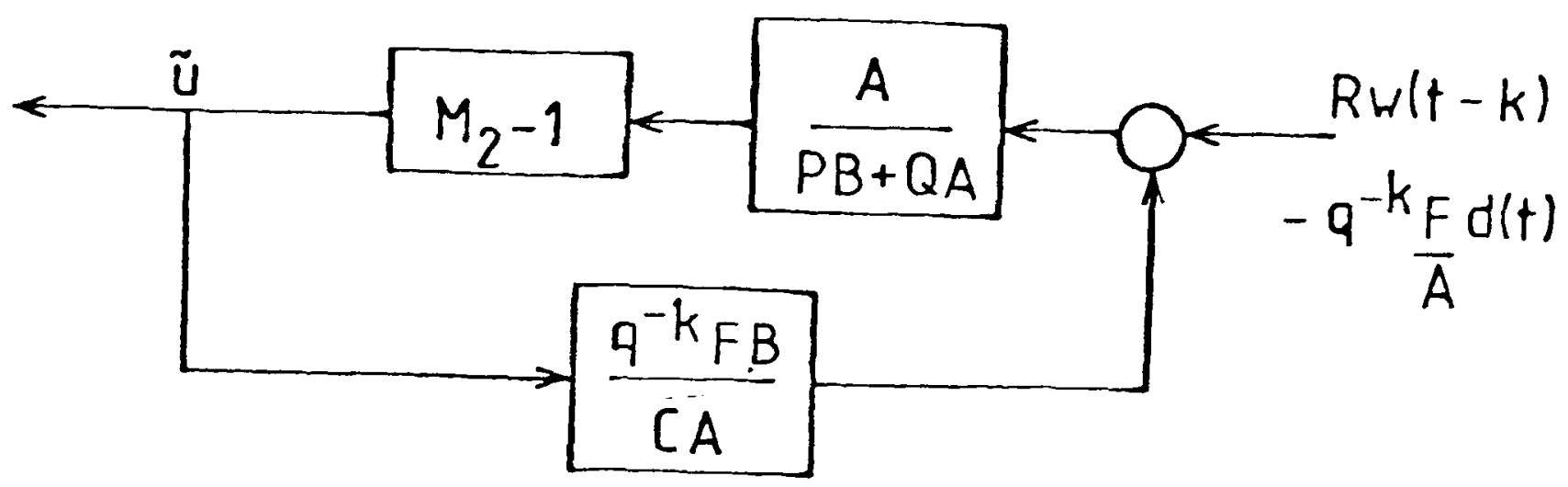

Fig. A-7 Simplified Feedback System for Input Error case

A.5 SECTION 2.4 .4

A.5.1 Derivation of Fig. 2-10, Section 2.4.4

$$
\begin{array}{ll}
Y=\left[M_{1}-1\right] Y & \text { :output error } \\
A Y(t)=B u(t-k)+C d(t) & \text { : nominal plant } \\
R_{1} B^{+} u(t)=C R W(t)-S_{A} Y_{0}(t) & \text { : control law }
\end{array}
$$

substituting Eqn. 21 in Eqn. 20 ,

$$
A R_{1} Y(t)=R_{1} C d(t)+q^{-k_{B}-} C R w(t)-q^{-k_{B}-s_{A}} Y-q^{-k_{B}-s_{A}} Y(t)
$$




\section{Appendix A}

but

$$
A R_{1}+q^{-k}{ }_{B}^{-} s_{A}=C A_{m} \quad \text { from Eqn. } 2-62
$$

Bence

$$
Y(t)=\frac{l}{A_{m}}\left[R B^{-} w(t-k)+R_{1} d(t)-q^{-k S^{-} B^{-}} q\right]
$$

Eqns. 19 and 22 together form the closed loop system of Fig. 2-10.

\section{A.5.2 Proof of Theorem 1, Section 2.4.4}

From Eqn. 2-62

$$
C A_{m}=A R_{1}+q^{-k_{B}-s_{A}}
$$

Let $\operatorname{deg}\left\{R_{1}\right\}=k-1$

and $\operatorname{deg}\left\{B^{-} S_{A}\right\}=\max \operatorname{deg}\left\{C A_{m^{\prime}} A_{1}\right\}-k$

Comparing with Eqn. 2-14 for the NA C-G STC, a particular solution of Eqn. 23 is (assuming that $A_{m}=P$ )

$$
\begin{aligned}
& R_{1}=E \\
& B^{-} S_{A}=F
\end{aligned}
$$

From Rucera[54], the general solution of $R_{1}$ and $B^{-} S_{A}$ may be written as

$$
\begin{aligned}
& R_{I}=E+q^{-k} U \\
& B^{-} s_{A}=F-A U
\end{aligned}
$$

where 0 is an arbitrary polynomial. Comparing Fig. 2-10 with Fig. 2-5 and Theorem 1 of section 2.4.1, it is obvious that a sufficient condition for the stability of the feedback system of Fig. 2-10 is that

$$
\sup _{|q|=1}\left|q^{-k \overrightarrow{B S}}\right|<\left[\operatorname{gain}\left\{M_{1}-1\right\}\right]^{-1}
$$

Comparing Eqn. 30 with set (a) of Theorem 1 of section 2.4.1, let

$$
q^{-k \overline{B S}} \overline{C A}=\frac{\tilde{q}^{-k} F B}{C(P B+Q A)}
$$

Eqn. 29 implies that for all solutions of $s_{A}$ in Eqn. 23, there exists $u$ such that $B^{-} S_{A}=F-A U$. substituting in Eqn. 31, we get 
Appendix A

$$
\frac{1}{2}=\frac{S}{\mathrm{~PB}}
$$

Hence $P B+Q A=\frac{P_{Q}{ }^{+}}{S_{A}}$

and only $B^{+}$, the stable zeros of $B$ are left in the zeros of $P B+Q A$.

\section{A.6 SECTION 2.4 .5}

$$
\begin{array}{ll}
9=\left(M_{1}-I\right) Y & \text { :output error } \\
A y(t)=B u(t-k)+d(t) & \text { :nominal plant } \\
A_{\mathbf{s}} Y(t)=B G_{\mathbf{s}} u(t-k)+G_{\mathbf{g}} d(t) &
\end{array}
$$

But from the control law of Egin. 2-65

$$
B G_{s} u(t)=B_{s} w(t-k)-B F_{s} Y_{0}(t)
$$

Substituting Eqn. 37 in 35

$$
\left(A_{s}+q^{-k} B F_{s}\right) Y(t)=B_{s} w(t-k)-q^{-k}{ }_{B F_{s}} g_{0}(t)+G_{s} d(t)
$$

But $A_{\mathbf{s}}+q^{-k_{B F}}=C A_{m}$

Bence $y(t)=\frac{I}{C A_{m}}\left[B B_{s} w(t-k)-q^{-k} B F_{s} g_{0}(t)+G_{s} d(t)\right]$

From Eqns. 34 and 35, we get the feedback system of Fig. 2-11, Section 2.4 .5 


\section{APPENDIX B}

\section{B.1 PROOFS FOR SECTION 3.1}

\section{B.1.1 Proof of Lemma 2}

Denote $\hat{e}_{1}(t) \exp (\alpha t)$ by $e_{1}(t)$ : output of estimator relationship, $\mathrm{H}_{\mathrm{cp}}$ $\hat{e}_{1}(t)+e_{p}(t)$ by $x(t)$ : input to $B_{c p}$

$x(t) \exp (\alpha t)$ by $x_{1}(t)$

Let suffixes, denote quantities corresponding to exponentially weighted input and output sequences to estimator, i.e.

$$
\begin{aligned}
x_{1}(t)= & x(t) \exp (\alpha t) \\
\dot{\theta}_{1}(t) & =\dot{\theta}_{1}(t-1)+s_{1}^{-1}(t-k) x_{1}(t-k) e_{1}(t) \\
e_{p_{1}} e_{1} & =x^{T}(t-k) \ddot{\theta}(t-1) \exp (\alpha t) e_{1}(t) \\
& =\exp (\alpha k) \ddot{\theta}^{T}(t-1) x_{1}(t-k) e_{1}(t)
\end{aligned}
$$

But $x_{1}(t-k) e_{1}(t)=x(t-k) \hat{e}_{1}(t) \exp (2 \alpha t) \exp (-\alpha k)$

$$
=s(t-k)[\dot{\theta}(t)-\dot{\theta}(t-1)] \exp (2 \alpha t) \exp (-\alpha k)
$$

The second equality follows from the estimator update, Eqn. 3-1 From Eqns. 2 and 3 ,

$$
\begin{aligned}
e_{p_{1}} e_{1} & =\ddot{\theta}^{\top}(t-1) s(t-k) \exp (2 \alpha t)[\dot{\theta}(t)-\dot{\theta}(t-1)] \\
& =\frac{1}{2}\left[\ddot{\theta}^{T}(t)+\ddot{\theta}^{T}(t-1)\right] s(t-k) \exp (2 \alpha t)[\ddot{\theta}(t)-\ddot{\theta}(t-1)] \\
& -\frac{1}{2}\left[\ddot{\theta}^{T}(t)-\ddot{\theta}^{T}(t-1)\right] s(t-k) \exp (2 \alpha t)[\ddot{\theta}(t)-\ddot{\theta}(t-1)]
\end{aligned}
$$

Let $s^{\prime}(t)=s(t) \exp (2 \alpha t)$ i.e. 
Appendix B

$$
S^{\prime}(t)=\beta \exp (2 \alpha) S^{\prime}(t-1)+x_{1}(t) x_{1}{ }^{T}(t) \text { :from Eqn. } 3-2
$$

From Eqns. 4 and 5

$$
\begin{aligned}
& \sum^{N}\left[2 e_{p 1} e_{1}+\sigma(t-k) e_{1}{ }^{2}(t)\right]=\sum^{N}\left[\ddot{\theta}^{T}(t) s^{\prime}(t-k) \exp (2 \alpha k) \ddot{\theta}(t)\right. \\
& \left.-\ddot{\theta}^{T}(t-1) S^{\prime}(t-k-1) \exp (2 \alpha k) \ddot{\theta}(t-1)\right]-\sum^{N} e_{p_{1}}^{2} \\
& +\exp (2 \alpha k) \sum^{N}[1-\beta \exp (2 \alpha)] \tilde{\theta}^{T}(t-1) s^{\prime}(t-k-1) \ddot{\theta}(t-1)
\end{aligned}
$$

If $1-\beta \exp (2 \alpha) \geqslant 0$, from Eqn. 6 we get

$$
\begin{aligned}
\sum^{N}\left[e_{p_{1}}{ }^{2}+2 e_{p_{1}} e_{1}\right. & \left.+\sigma(t-k) e_{1}{ }^{2}\right]+\exp (2 \alpha k) \ddot{\theta}(k) S \cdot(0) \tilde{\theta}(k) \\
& >\exp (2 \alpha k) \theta^{-T}(N) S \cdot(N-k) \ddot{\theta}(N) \quad \forall N>t_{0}
\end{aligned}
$$

Eqn. 7 is similar to the dissipative inequality of Eqn. 3-6 and Lemma 2 follows. If $\alpha=0$, then the proof above reduces to that for Lemma 1.

\section{B.1.2 Proof of Lemma 3}

$$
\text { If } x(t) \in I_{\infty} \text {, then }
$$

$$
\begin{aligned}
\sum^{N}|x(t) \exp (\alpha t)|^{2} & =\|x\|_{\infty}^{2} \sum^{N}|\exp (2 \alpha t)| \\
& =\| x_{\infty} \frac{{ }^{2} \exp [2 \alpha(N+1)]-1}{\exp [2 \alpha]-1} \forall \text { finite } N>t_{0}
\end{aligned}
$$

Applying Lemma 2, Section 3.1, when $1-\beta \exp (2 \alpha)>0$

$$
\sum \hat{e}_{1}{ }^{2}(t) \exp (2 \alpha t)<\left(1-\sigma_{0}\right)^{-1}\|x\|_{\infty}{ }^{2} \frac{\exp [2 \alpha(N+1)]-1}{\exp [2 \alpha]-1}
$$

But $\hat{e}_{1}^{2}(N) \exp (2 \alpha N)<\sum \hat{e}_{1}^{2}(t) \exp (2 \alpha t)$

Bence $\hat{e}_{1}^{2}(N)<\left(1-\sigma_{0}\right)^{-1} \operatorname{lxl}_{\infty}^{2} \frac{\exp [2 \alpha]-\exp [-2 \alpha N]}{\exp [2 \alpha]-1}$ 
Appendix B

$$
\leqslant\left(1-\sigma_{0}\right)^{-1}\|x\|_{\infty}^{2} \quad \forall N>t_{0}
$$

\section{B.1.3 Proof of Lemma 4}

For convenience, the suffix 0 is dropped from the data vector

(i) $\hat{e}_{0}=\Phi(t)-x^{T}(t-k) \hat{\theta}(t)$

substituting $\hat{\theta}(t)=\hat{\theta}(t-1)+s^{-1}(t-k) x(t-k) \hat{e}_{1}(t)$

$\hat{e}_{0}=\Phi(t)-x^{T}(t-k) \hat{\theta}(t-1)-\sigma(t-k) \hat{e}_{1}(t)$

$=\hat{e}_{1}(t)-\sigma(t-k) \hat{e}_{1}(t)$

(ii) $\hat{e}_{0}+e_{p}^{0}=\Phi(t)-x^{T}(t-k) \hat{\theta}(t)+x^{T}(t-k) \tilde{\theta}(t)$

$=\Phi(t)-x^{T}(t-k) \theta$

$=\Phi(t)-x^{T}(t-k) \hat{\theta}(t-1)+x^{T}(t-k) \tilde{\theta}(t-1)$

$=\hat{e}_{1}(t)+e_{p}(t)$

(iii) From Lemma 1, Section 3.1

$$
\sum\left[\left(e_{p}+\hat{e}_{1}\right)^{2}+[\sigma(t-k)-1] \hat{e}_{1}{ }^{2}\right] \geqslant 0: \forall N>t_{0}
$$

substituting (i) and (ii) above

$\sum\left(e_{p}^{0}+\hat{e}_{0}\right)^{2} \geqslant \sum \hat{e}_{0}^{2} \forall N>t_{0}$

$\square$

B. 2 PROOF OF THEOREM $1(B)$, SECTION 3.2 .2

In Fig. 3-3, let

$\operatorname{cose}(t)=\nabla(t)$

and $\left(C_{d} \hat{C}-1\right) \hat{e}_{1}(t)=z(t)$

Consider an associated system consisting of $(\bar{c} / \hat{C}-1)^{*}$ mapping $\hat{e}_{1}(t) \exp (\alpha t)$ to $z(t) \exp (\alpha t)$ and $H_{c p}{ }^{*}$ of Lemma 2 , section 3.1 . since Co -1 is a linear, time-invariant ARMA operator, we can write the 
Appendix B

relationship between $z(t)$ and $\hat{e}_{1}(t)$ as

$$
\begin{aligned}
\begin{aligned}
\hat{c} z(t)= & \left(c_{0}-\hat{c}\right) \hat{e}_{1}(t)=\ddot{c}_{\hat{e}_{1}}(t) \\
\text { ie. } z(t) & +\hat{c}_{1} z(t-1)+\hat{c}_{2}(t-2)+\ldots \\
& =\tilde{c}_{1} \hat{e}_{1}(t-1)+\tilde{c}_{2} \hat{e}_{1}(t-2)+\ldots
\end{aligned} \\
\text { Hence } z(t) \exp (\alpha t)+\hat{c}_{1} \exp (\alpha) z(t-1) \exp [\alpha(t-1)]+\ldots \\
=\tilde{c}_{1} \exp (\alpha) \hat{e}_{1}(t-1) \exp [\alpha(t-1)]+\ldots
\end{aligned}
$$

If Eqn. 16 is regarded as a mapping from $z(t) \exp (\alpha t)$ to $\hat{e}_{1}(t) \exp (\alpha t)$, we can write the corresponding ARMA model as

$$
\left[c_{0} \hat{C}\left([\exp (-\alpha) q]^{-1}\right)-1\right]=\left(c_{b} \hat{C}-1\right)^{*}
$$

If $v(t) \epsilon 1_{\infty}$, then $c_{b} \hat{C} e(t) \exp (\alpha t) \in 1_{2 e}$. From Lemma 2, section 3.1, $\mathrm{H}_{\mathrm{cp}}{ }^{\mathrm{m}}$ maps the exponentially weighted quantities with gain $\left(1-\sigma_{0}\right)^{-1 / 2}$. Applying the small gain theorem to the associated system, we get

$$
\begin{aligned}
& \left|\hat{e}_{1}(N)\right|^{2} \exp (2 \alpha N) \leqslant \sum\left|\hat{e}_{I}\right|^{2} \exp (2 \alpha t) \\
& \leqslant\left[\frac{y}{1-y^{1}}\right]^{2} \sum|v(t)|^{2} \exp (2 \alpha t) \\
& \leqslant\left[\frac{y}{1-y}\right]^{2} \nabla(t) \|_{\infty}^{2} \frac{\exp [2 \alpha(N+1)]-1}{\exp [2 \alpha]-1} \\
& \text { Hence }\left|\hat{e}_{1}{ }^{2}(N)\right|<\left[\frac{\gamma}{1-\gamma}\right]^{2}\|v(t)\|_{\infty}^{2} \quad \forall N>t_{0} \\
& \text { where } \gamma^{2}=\left(1-0_{0}\right)^{-1}(c / \hat{C})^{*}-1 \|^{2} \\
& \text { and } y_{1}^{2}=\left(1-0_{0}\right)^{-1}
\end{aligned}
$$

B. 3 PROOF OF LEMMA 1 , SECTION 3.4 .1

Lemma

Let $M$ be a nonlinear, continuously Freshet differentiable ( $C^{1}$ ) map from $I_{2}$ to $I_{2}$ and $I$ be a linear mapping from $I_{2}$ to $I_{2}$ such that $L^{-1}$ exists and

- $206-$ 


\section{Appendix B}

is stable. Assume that $\mathrm{LML}^{-1}$ maps 0 to 0 . Then

$$
\left\|\mathrm{IML}^{-1}\right\|=\|M\|_{\Delta}
$$

\section{Proof}

Let $x \in 1_{2 e}$ and $y \equiv \operatorname{LML}^{-1}(x)$

By Taylor's Theorem,

$$
y(x)=y(0)+\int_{0}^{1} D\left\{\mathrm{um}^{-1}(\alpha x)\right\} x d \alpha
$$

where $D(\cdot(\alpha x)\}$ denotes the Frechet derivative evaluated at $\alpha x$.

Since $Y(0)=0$,

$$
\begin{aligned}
\|y(x)\| & \leqslant \sup _{\alpha}\left\|\left\{\operatorname{IML}^{-1}\right\}\right\|\|x\|, 0 \leqslant \alpha \leqslant 1 \\
\sup _{\forall x} \frac{\|y\|}{\|x\|} & \sup _{\alpha}\left\|\mathrm{D}\left(\mathrm{LML} L^{-1}\right\}\right\|, \\
& =\sup _{\alpha} \| \mathrm{LD}(\mathrm{M}) \mathrm{L}^{-1}
\end{aligned}
$$

But $D\{M\}$ is linear, hence $L D\{M\} L^{-1}=D(M)$

From Willems $[88],\|D\{M\}\|=\|M\|_{\Delta}$.

\section{B. 4 PROOFS FOR SECTION 3.5}

\section{B.4.1 Proof of Theorem 1}

$$
\text { Define: } \begin{aligned}
x_{1}(t) & \triangleq e(t)-e_{m}(t) \\
x_{2}(t) & \cong e_{1}(t)+\hat{e}_{1}(t)
\end{aligned}
$$

Assume that $e_{m}(t) \in I_{2}$. This implies that the input to $\mathrm{H}_{0}, \mathbf{x}_{2} \epsilon_{\mathbf{I}_{2}}{ }^{\prime}$. From Eqn. $18,\left\|\hat{e}_{1}\right\|^{2}=\left\|x_{2}-e_{1} \cdot\right\|^{2}=\left\|x_{2}\right\|^{2}+\left\|e_{1} \cdot\right\|^{2}$

$$
\text { because } x_{2} \in I_{2}, e_{1}^{\prime} \epsilon 1_{2}^{\perp} \quad \text { implies } \quad \sum_{x_{2}}^{\infty} e_{1}^{\prime}=0
$$

For $x_{2} \in I_{2} \cdot H_{0}$ has a gain given as usual by

$$
\gamma_{1}=\sup _{|q|=1}\left|\mathrm{~B}_{0}\right|
$$

Let the gain of $\mathrm{H}_{\mathrm{cp}}=\left(1-\sigma_{0}\right)^{-1 / 2}=\gamma_{2}$

Let $\gamma=\gamma_{1} \gamma_{2}$ 
From Fig. 3-12,

$$
\begin{aligned}
& \left\|\hat{e}_{1}\right\|^{2}<\left\{y_{2}\left[\|u+\| e_{m} \|\right]\right\}^{2} \\
& <\gamma_{2}{ }^{2} \operatorname{le}\left\|^{2}+\left(\gamma_{1} \gamma_{2}\right)^{2} x_{2}\right\|^{2}+2 y_{2} \gamma_{2}^{2} \text { le } \| x_{2}
\end{aligned}
$$

(where we use $\left\|e_{m}\right\| \gamma_{1}\left\|_{2}\right\|$ )

substituting Eqn. 23 in Eqn. 19 and re-arranging,

$$
\left\|e_{1} \cdot\right\|^{2}<-\left(1-y^{2}\right)\left\|x_{2}\right\|^{2}+2 \gamma_{1} \gamma_{2}^{2}\left\|x_{2}\right\| e\left\|+y_{2}^{2}\right\| e \|^{2}
$$

Note that $\gamma<1$ by assumption. For a fixed $e \epsilon I_{2}$, the maximum value of the RHS of Eqn. 24 is $\gamma_{2}{ }^{2}$ "e ${ }^{2}\left(1-\gamma^{2}\right)^{-1}$. Hence if $e_{1}$ ' is chosen such that

$$
e_{1} \cdot>\gamma_{2}\left(1-\gamma^{2}\right)^{-1 / 2} \mid e \text { i }
$$

Eqn. 24 is false. This implies $e_{m}(t) \in I_{2}$.

Bence $x_{2} \in 1_{2}$ and $\hat{e}_{1}(t) \notin 1_{2}$ and $\delta=y_{2} /\left[1-y^{2}\right]^{-1 / 2}$

b) $1_{\infty}$ instability

Let $e_{1} \cdot \epsilon I_{\infty}$ and choose $\alpha<0$ such that

$$
e_{1} \cdot^{*}=e_{1} \cdot(t) \exp (\alpha t) \in I_{2}^{\perp} \text { for the associated system } H_{0}^{*} \text {. }
$$

Consider the associated feedback system formed by $\mathrm{H}_{0}^{*}$ and $\mathrm{H}_{\mathrm{CP}}^{*}$ If $\mathrm{e}_{1}^{.^{*}}$ and $e^{*}$ are selected such that the associated system is $I_{2}$ unstable i.e. by specifying conditions on them similar to those for part (a) above, then $\hat{\mathrm{e}}_{1}{ }_{1} \mathrm{I}_{2}$.

But $\hat{e}_{1}(t)=\hat{e}_{1}{ }^{\star} \exp (-\alpha t)$. Hence if the sequence $\hat{e}_{1}^{\star} t I_{2}$. $\hat{e}_{1}(t) \in 1_{\infty}$. 


\section{Appendix B}

\section{B.4.2 Proof of Lemma 1}

The proof works by contradiction. Consider Fig. B-l below.

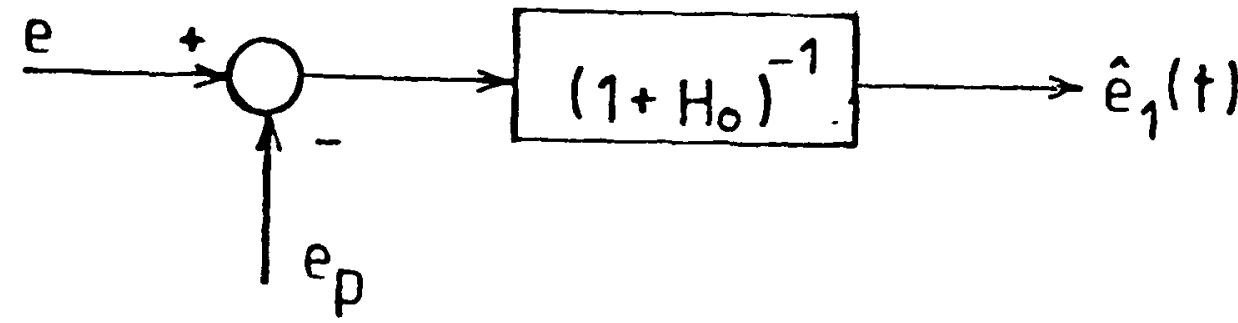

$$
\begin{array}{ll}
\text { Fig. R-1 Relationship between } & \\
& \text { Error quantities }
\end{array}
$$

From Corollary 2.3, section 2.4.1, the nonadaptive self-tuner with nominal plant $\theta^{\star}$ is unstable if $\left[1+{ }_{0}\left(\theta^{\star}\right)\right]^{-1}$ has poles in the unstable region.

Assume that $\hat{e}_{1} \epsilon 1_{2}$. This implies that $x_{0}(t)$ is uniformly bounded and hence

$$
\lim _{t \rightarrow \infty} e_{p}=x_{0}^{T}(t-k)\left[\hat{\theta}(t-1)-\theta^{\star}\right]=0
$$

But if $e(t)$ is chosen to be in $I_{2}^{1}\left(\left(1+B_{0}\right)^{-1}\right\}$, then

$\lim _{t \rightarrow \infty} \hat{e}_{1}(t) E I_{2}$, which contradicts the assumption above.

$\mathbf{0}$

\section{B. 5 PROOFS FOR SECTION 3.7}

\section{B.5.1 Proof of Lemma 1}

a) From the matrix gain update, Eqn. 3-2,

$$
S(t)=\beta S(t-1)+x(t) X^{T}(t)
$$

Applying the matxix inversion lemma,

$$
\beta S^{-1}(t)=s^{-1}(t-1)-\left[\beta+\sigma_{1}(t)\right]^{-1}\left[s^{-1}(t-1) x(t) x^{T}(t) s^{-1}(t-1)\right](26)
$$

From the definition of $\sigma$ in Eqn. $3-91$ and defining $\sigma_{1}(t)$ to be

$$
\sigma_{1}(t) \triangleq x^{T}(t) s^{-1}(t-1) x(t)
$$


Appendix B

we get $\sigma(t)=\sigma_{1}(t)\left[\beta+\sigma_{1}(t)\right]^{-1}$

(27)

Since $x$ is uniformly bounded, $\sigma<1$. 


\section{Appendix B}

B.6 PROOFS FOR SECTION 3.8

\section{B.6.1 Proof of Lemmp 1}

Denote $e_{p}(t) s^{-1 / 2}(t-k)$ by $\bar{e}_{p}$ and $\hat{e}_{1}(t) s^{-1 / 2}(t-k)$ by $\bar{e}_{1}$. similaxly, let $\left(\hat{e}_{1}+e_{p}\right) s^{-1 / 2}=\left(e-e_{m}\right) s^{-1 / 2}=\bar{x}_{1}$.

From the scalar gain estimator update, Eqn. 3-102

$$
\dot{\theta}(t)=\tilde{\theta}(t-1)+s^{-1}(t-k) x(t-k) \hat{e}_{1}(t)
$$

Bence,

$$
\begin{aligned}
\vec{e}_{p} \bar{e}_{1}= & \ddot{\theta}^{T}(t-1) s^{-1}(t-k) x(t-k) \hat{e}_{1}(t) \\
= & \ddot{\theta}^{T}(t-1)[\ddot{\theta}(t)-\dot{\theta}(t-1)] \\
= & \frac{1}{2}[\dot{\theta}(t)+\dot{\theta}(t-1)]^{T}[\dot{\theta}(t)-\dot{\theta}(t-1)] \\
& \quad-\frac{1}{2}[\dot{\theta}(t)-\dot{\theta}(t-1)]^{T}[\dot{\theta}(t)-\dot{\theta}(t-1)]
\end{aligned}
$$

Therefore,

$$
\begin{aligned}
& \sum_{\text {where } \sigma_{s}(t) \leqq x^{T}(t) x(t) s^{-1}(t)<1}^{N}\left[2 \sigma_{s} \bar{e}_{1}^{2}\right]=\dot{\theta}^{T}(N) \dot{\theta}(N)-\dot{\theta}^{T}(0) \dot{\theta}(0) \\
& \text { whe }
\end{aligned}
$$

From Eqns. 36 and 37

$$
\begin{gathered}
\sum_{2} 2 \bar{e}_{p} \bar{e}_{1}+\sigma_{s} \bar{e}_{1}^{2}=\sum^{N}\left[\left(\bar{e}_{p}+\bar{e}_{1}\right)^{2}-\bar{e}_{p}^{2}+\left(\sigma_{s}-1\right) \bar{e}_{1}^{2}\right] \\
<\sum_{N}^{N}\left[\left(\bar{e}_{p}+\bar{e}_{1}\right)^{2}-\bar{e}_{p}^{2}\right]
\end{gathered}
$$

Bence,

$$
\sum \bar{e}_{p}^{2}<\sum\left[\bar{e}_{1}^{N}+\bar{e}_{p}\right]^{2}+\text { constant } \forall N>t_{0}
$$




\section{Appendix B}

B.6.2 Proof of Lemma 2

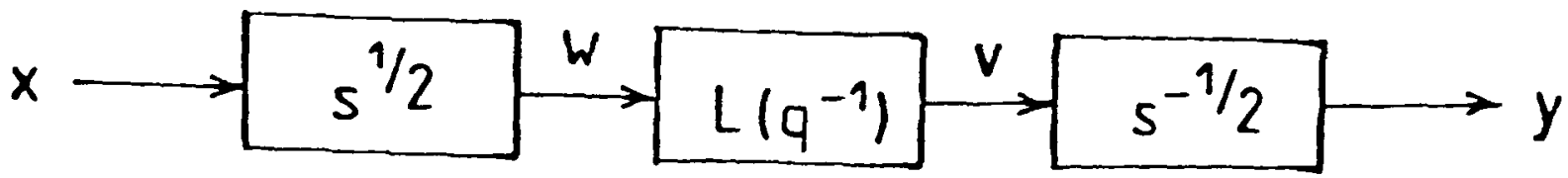

Fig. B-2 Operator with scalar weighting

Let $I$ be a bounded mapping from $I_{2}$ to $I_{2}$ i.e.

$$
\sum^{N} v^{2}(t)<v^{2} \sum^{N} w^{2}(t) \quad v N>t_{0}
$$

with reference to Fig. B-2,

$$
\begin{aligned}
& v^{2}(t)=s(t) y^{2}(t) \\
& w^{2}(t)=s(t) x^{2}(t)
\end{aligned}
$$

Let $s(t)$ be a positive non-decreasing scalar sequence. Then from Eqn. 39

$$
\sum^{N} s(t)\left[y^{2}(t)-\gamma^{2} x^{2}(t)\right] \leqslant 0 \quad \forall N>t_{0}
$$

since $s(t)$ is increasing, $s^{-1}(0) \geqslant s^{-1}(I) \geqslant \ldots . s^{-1}(N)>0$.

Using Abel's Lemma (Titchmarsh[82]), we get from Eqn. 42

$$
\sum^{N}\left[y^{2}(t)-\gamma^{2} x^{2}(t)\right] \leqslant 0 \quad \forall N>t_{0}
$$

\section{B.6.3 Proof of Theorem 1}

If $e, e_{1}^{\prime} \epsilon I_{2}$ and $s$ is a positive, non-decreasing sequence, then the conditions of the theorem meet the small gain condition, and ensure that $\hat{e}_{1} s^{-1 / 2}(t-k) \epsilon I_{2} i . e$.

$$
\sum_{1}^{\infty} \hat{e}_{1}^{2}(t) s^{-1}(t-k)<\infty
$$

Using Kronecker's Iremma, we get

$$
\lim _{N \rightarrow \infty} \frac{N}{s(N-k)} \frac{1}{N} \sum_{\hat{e}_{1}^{2}}^{R}(t)=0
$$

since the plant is assumed to be stably invertible, there exists 


\section{Appendix B}

constants $K_{1}, K_{2}$ such that

$$
\frac{s(N)}{N}=\frac{1}{N} \sum^{N} \beta^{N-i} x^{T} x<\frac{K}{N} \sum \sum y^{2}(t)+K_{2}
$$

But $\hat{e}_{1}(t)=\Phi(t)-\hat{\Phi}(t / t-1)=\Phi(t)-R w(t-1)$

Bence $Y(t)=\frac{1}{p}\left[\hat{e}_{1}(t)+R w(t-1)\right]$

which implies that

$$
\frac{1}{N} \sum^{N} y^{2}(t)<\frac{\pi}{N} \sum^{N} \hat{e}_{1}^{2}(t)+\frac{\pi}{N} 4 \sum^{N}[R w(t-1)]^{2}
$$

Substituting Eqn. 48 in Eqn. 45

$$
\frac{s(N)}{N}<\frac{K}{N} 5 \sum_{1}^{N} \hat{e}_{1}^{2}(t)+K_{6}
$$

and substituting Eqn. 49 in 44 we get

$$
\lim _{N \rightarrow \infty} \frac{1}{N} \sum^{N} \hat{e}_{1}{ }^{2}(t)=0
$$

From Eqn. 46, this implies

$$
\lim _{N \rightarrow \infty} \frac{1}{N} \sum^{N}[\Phi(t)-R w(t-1)]^{2}=0
$$

B.7 PROOF OF LEMMA 1, SECTION 3.9

The proof will be shown for the exror feedback system of Fig. 3-7, being very similar for the other cases. Fig. 3-7 is equivalent to Fig. B-3 below.

Since gain $\left\{t^{-1 / 2} B_{0} t^{1 / 2}\right\}=$ gain $\left\{B_{0}\right\}$

and gain $\left\{t^{-1 / 2}{ }_{C P} t^{1 / 2}\right\}=$ gain $\left(B_{C P}\right\}$ by Lemma 2 , Section 3.8

Then the conditions of Theorem $1(a)$, Section 3.4.1 ensure that

$$
\begin{aligned}
& \sum^{\infty} \hat{e}_{1}{ }^{2} t^{-1}<\infty \\
& \quad \text { if } \sum^{\infty} e^{2} t^{-1}<\infty \text { and } \sum^{\infty} e_{1}^{12} t^{-1}<\infty .
\end{aligned}
$$

By Kronecker's Lemma, 
Appendix B

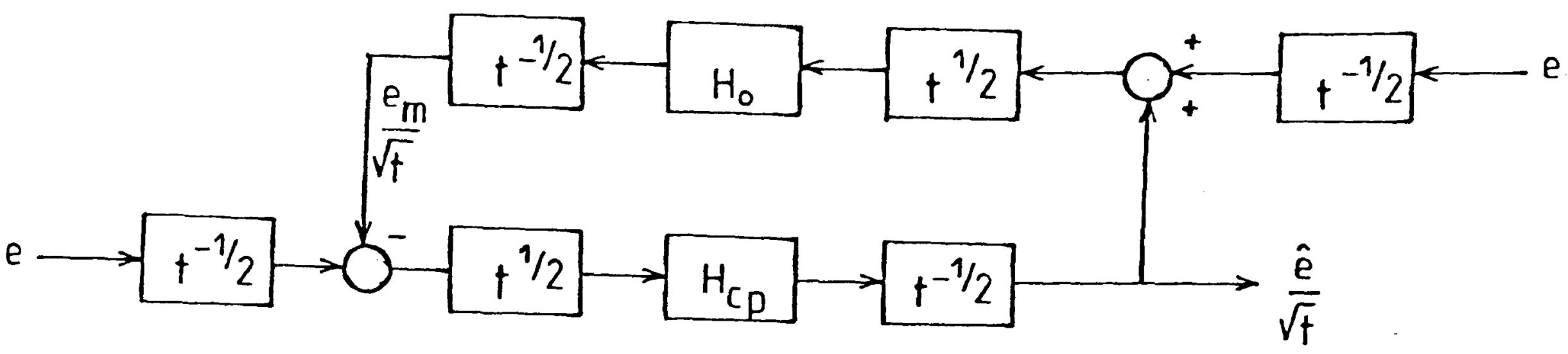

Fig. E-3 Feedback system with time as weighting sequence $\lim _{N \rightarrow \infty} \frac{1}{N} \sum_{1}^{N} \hat{e}_{1}^{2}=0$ if the initial conditions are bounded.

ㅁ 


\section{APPENDIX C}

\section{C.1 PROOF OF IEMMA 2 , SECTION 4.1 .1}

The equations describing the multiple recursion estimator, with a matrix gain are:

$$
\begin{gathered}
\hat{\theta}(t)=\hat{\theta}(t-k)+s^{-1}(t-k) x(t-k) \hat{e}_{k}(t) \\
s(t-k)=\beta s(t-2 k)+x(t-k) x^{T}(t-k) \\
\text { Define } e_{p}^{k}(t) \cong x^{T}(t-k) \tilde{\theta}(t-k)
\end{gathered}
$$

From Eqn. 3 and the definitions of Chapter 2, it is easy to show that

$$
e_{p}^{k}+\hat{e}_{k}=e_{p}+\hat{e}_{1}
$$

From Eqns. 1 to 3 ,

$$
\begin{aligned}
& e_{p}^{k} \hat{e}_{k}=\tilde{\theta}^{T}(t-k) x(t-k) \hat{e}_{k}(t) \\
& =\ddot{\theta}^{T}(t-k) S(t-k)[\ddot{\theta}(t)-\ddot{\theta}(t-k)] \\
& =\frac{1}{2}[\dot{\theta}(t)+\dot{\theta}(t-k)]^{T} s(t-k)[\dot{\theta}(t)-\dot{\theta}(t-k)] \\
& -\frac{1}{2}[\ddot{\theta}(t)-\tilde{\theta}(t-k)]^{T} s(t-k)[\tilde{\theta}(t)-\tilde{\theta}(t-k)] \\
& =\frac{1}{2}[\dot{\theta}(t)+\dot{\theta}(t-k)]^{T} s(t-k)[\ddot{\theta}(t)-\dot{\theta}(t-k)] \\
& -\frac{1}{2} \sigma(t-k) \hat{e}_{k}^{2}(t)
\end{aligned}
$$

and

$$
\begin{aligned}
& \sum\left[2 e_{p}^{k N}+\sigma(t-k) \hat{e}_{k}{ }^{2}\right]= \\
& \quad \sum^{N}\left[\dot{\theta}^{-T}(t) s(t-k) \dot{\theta}(t)-\ddot{\theta}^{-T}(t-k) s(t-2 k) \theta^{T}(t-k)\right]
\end{aligned}
$$


Appendix C

$$
+\sum^{N} \tilde{\theta}^{T}(t-k)[s(t-2 k)-s(t-k)] \ddot{\theta}(t-k) \forall N>t_{0}
$$

Using Eqn. 3 above and the last equality, we get (since $\beta<1$ )

$$
\begin{aligned}
\sum & {\left[e_{p}^{k_{2}}+2 e_{p}^{k} \hat{e}_{k}+\sigma(t-k) \hat{e}_{k}{ }^{2}\right] } \\
& +\ddot{\theta}^{-T}(k) S(0) \ddot{\theta}(k)+\ldots+\ddot{\theta}^{-T}(2 k-1) s(k-1) \ddot{\theta}(2 k-1) \geqslant 0
\end{aligned}
$$

Following Lemuas 1 and 2 of section 3.1, it can then be shown that $\sum \hat{e}_{k}^{2}(t)<\left(1-\sigma_{0}\right)^{-1} \sum^{N}\left[\hat{e}_{k}+e_{p}^{k}\right]^{2}+$ constants $\forall N>t_{0}$

\section{C.2 PROOF OF LEMMA 1, SECTION 4.1.2}

The proof will be in two parts: with reference to Fig. 4-1, the gain of the $j$ th element of $\mathbf{B}_{\mathbf{e}^{\prime}} \mathbf{u}_{\mathbf{e}^{j}}^{j}$ will be shown to be $\gamma_{j}$, where

$$
\gamma_{j}{ }^{2}=\sigma_{0} \sigma_{2}^{3}(j-1)
$$

and $o_{2}^{\mathbf{s}}(i)$ will then be evaluated.

$\mathbf{H}_{e}^{j}$ denotes the functional relationship between $\left(\hat{e}_{j}-\hat{e}_{j-1}\right)$ and $\hat{e}_{1}$. By definition,

$$
\begin{aligned}
& \hat{e}_{j}(t)=\Phi(t)-x^{T}(t-k) \hat{\theta}(t-j) \\
& \hat{e}_{1}(t)=\Phi(t)-x^{T}(t-k) \hat{\theta}(t-1)
\end{aligned}
$$

In this section, the suffix for $x$ will be dropped for convenience. Bence,

$$
\begin{aligned}
\hat{e}_{j}-\hat{e}_{j-1} & =x^{T}(t-k)[\hat{\theta}(t+1-j)-\hat{\theta}(t-j)] \\
& =x^{T}(t-k) s^{-1}(t+1-j-k) x(t+1-j-k) \hat{e}_{1}(t+1-j)
\end{aligned}
$$

The second equality follows from the estimator equations (Eqns. 3-1 to 3-3).

$$
\begin{gathered}
{\left[\hat{e}_{j}-\hat{e}_{j-1}\right]^{2}=\hat{e}_{1}{ }^{2}(t+1-j) t \operatorname{race}\left[s^{-1}(t+1-j-k) x(t+1-j-k) x^{T}(t+1-j-k)\right.} \\
\left.s^{-1}(t+1-j-k) x(t-k) x^{T}(t-k)\right]
\end{gathered}
$$




\section{Appendix C}

Note that $s^{-1}$ is positive semi-definite, and $s^{-1} \mathrm{xx}^{\mathrm{T}}$ is positive semi-definite of rank 1 . It can be shown that if $A$ and $B$ are positive semi-definite matrices, each of rank 1 , then

\section{trace $[A B]<[$ trace $A][$ trace $B]$}

Applying Eqn. 10 to Eqn. 9 and bearing in mind the definitions of $\sigma$ and $\sigma_{2}$ in Section 4.1 , we get

$$
\left[\hat{e}_{j}-\hat{e}_{j-1}\right]^{2}<\hat{e}_{1}{ }^{2}(t+1-j) \sigma(t+1-j-k) \sigma_{2}(t+1-j-k, j-1)
$$

which implies that

$$
\begin{aligned}
& \sum^{\mathbb{N}}\left[\hat{e}_{j}-\hat{e}_{j-1}\right]^{2}<\sigma_{0} \sigma_{2}^{s}(j-1) \sum \hat{e}_{1}{ }^{2}(t) \quad \forall>t_{0} \\
& \text { where } \sigma_{2}^{s}(j-1)=\sup _{t>t_{0}} \sigma_{2}(t, j-1)
\end{aligned}
$$

In the next part of the proof, it will be shown that $\sigma_{2}^{\mathbf{s}}(j)$ can be evaluated recursively as

$$
\sigma_{2}^{s}(j) \leq \frac{\beta \sigma}{1-\sigma} 0\left[\beta^{j-1}+\beta^{j-1} \sigma_{2}^{s}(1)+\ldots+\sigma_{2}^{s}(j-1)\right]
$$

where $\beta$ is the forgetting factor.

From the definitions in section 4.1,

$$
\begin{aligned}
& \sigma_{2}(t, j)[1-\sigma(t+j)] \\
& =x^{T}(t+j)\left[s^{-1}(t)-s^{-1}(t) x(t+j) x^{T}(t+j) s^{-1}(t+j)\right] x(t+j) \\
& =x^{T}(t+j)\left[s^{-1}(t)-s^{-1}(t)[s(t+j)-\beta s(t+j-1)] s^{-1}(t+j)\right] x(t+j) \\
& =\beta x^{T}(t+j) s^{-1}(t) s(t+j-1) s^{-1}(t+j) x(t+j)
\end{aligned}
$$

where the second equality made use of the estimator matrix gain update equation

$$
\begin{aligned}
s(t+j-1) & =\beta S(t+j-2)+x(t+j-1) x^{T}(t+j-1) \\
& =\beta^{j-1} s(t)+\beta^{j-2} x(t+1) x^{T}(t+1)+\ldots+t+x(t+j-1) x^{T}(t+j-1)
\end{aligned}
$$

Substituting Eqn. 16 in Eqn. 15, and using Eqn. 10 above, we get

$$
\sigma_{2}(t, j)[1-\sigma(t+j)]
$$


Appendix C

$$
<\beta O(t+j)\left[\beta^{j-1}+\beta^{j-1} \sigma_{2}(t, 1)+\ldots+\sigma_{2}(t, j-1)\right]
$$

The result of Eqn. 14 follows.

C.3 PROOF OF LEMMA 1 , SECTION 4.3 .1

Define $\mu \cong I-\beta_{\mathrm{dc}}\left(\beta_{\mathrm{dc}}\right.$ is the forgetting factor for the offset estimator)

$$
\begin{aligned}
& \dot{d}(t) \cong \hat{d}(t)-\bar{d} \\
& e_{1}(t) \triangleq \hat{e}_{1}(t)-\hat{d}(t-1) \\
& e_{p}(t) \triangleq e_{p}(t)+\tilde{d}(t-1)
\end{aligned}
$$

From Eqn. 4-28,

$$
\dot{\theta}(t)=\dot{\theta}(t-1)+s^{-1}(t-k) x(t-k) e_{1}(t)
$$

From Eqn. 4-29

$$
\dot{d}(t)=\tilde{d}(t-I)+\mu e_{1}(t)
$$

Let $X$ (the suffix o has been dropped for convenience) and $\theta$, of dimension $n$ be augmented by $0^{\prime} \cdot{ }^{\prime}$. Denote the augmented vectors by $X^{\prime}$ and $\theta$, of dimension $(n+1)$. Let $J$ be a constant vector of dimension $(n+1)$ and

$$
\mathbf{J}^{\mathbf{T}}=[0,0, \ldots \ldots, 0,1]
$$

Eqn. 19 can then be written as

$$
\dot{D}(t)=\tilde{D}(t-1)+\mu J e_{1}(t)
$$

where $\ddot{D}(t)$ is also an $(n+1)$ vector and $\ddot{D}(0)=0$.

Define $x_{1} \cong x^{\prime}+J$

$$
\text { and } \dot{\theta}_{1} \equiv \tilde{\theta}^{\prime}+\tilde{D}
$$

Then

$$
\begin{aligned}
& x_{1}{ }^{T}(t-k) \tilde{\theta}_{1}(t-1)=[x \cdot(t-k)+J]^{T}[\tilde{\theta} \cdot(t-1)+\tilde{D}(t-1)] \\
& =x^{\cdot T}(t-k) \tilde{\theta} \cdot(t-1)+J^{T} \tilde{D}(t-1) \\
& =x^{T}(t-k) \tilde{\theta}(t-1)+\tilde{d}(t-1) \\
& =e_{p} .
\end{aligned}
$$




\section{Appendix C}

Let $s^{\prime}(t)$ be the matrix corresponding to $x^{\prime}$ i.e.

$$
S^{\prime}(t)=\beta S^{\prime}(t-1)+X \cdot(t) X \cdot T(t)
$$

Egns. 18 and 19 can then be combined as

$$
\begin{aligned}
\dot{\theta}_{1}(t) & =\dot{\theta}_{1}(t-1)+\left[s^{\prime-1}(t-k) x \cdot(t-k)+\mu J\right] e_{1}(t) \\
& =\dot{\theta}_{1}(t-1)+s_{1}^{-1}(t-k) x_{1}(t-k) e_{1}(t)
\end{aligned}
$$

where $s_{1}^{-1}(t)=s^{-1}(t)+\mu J J^{T}$

since $s_{2}, s^{\prime}, J J^{T}$ are symmetric positive semi-definite and $J^{T}$ is filled with 0 's except for the $(n+1, n+1)$ element, it can be shown by matrix partitioning that

$$
s_{1}(t)=s^{\prime}(t)+\frac{1}{\mu} J J^{T} \quad: \mu \neq 0
$$

so that

$$
s_{1}(t)-s_{1}(t-1)=s^{\prime}(t)-s^{\prime}(t-1)
$$

Note that Eqn. 22 is in the form of Eqn. 3-1. From Eqns. 20 to 24, it is straightforward to show that for this combined estimator, the equation corresponding to Eqn. $3-6$ is

$$
\begin{aligned}
& \sum\left[e_{p} \cdot{ }^{2}(t)+2 e_{p} \cdot(t) e_{1}(t)+\sigma^{d}(t-k) e_{1}{ }^{2}(t)\right] \\
& \quad+\dot{\theta}_{1}(k) s_{1}(0) \dot{\theta}_{1}(k) \geqslant \dot{\theta}_{1}(N) s_{1}(N-k) \dot{\theta}_{1}(N) \forall N>t_{0}
\end{aligned}
$$

where $\sigma^{d}(t)=1-\beta_{d c}+\sigma(t)$

and $\sigma(t)=x^{T}(t) s^{-1}(t) x(t):$ as in section 3.1

By analogy to Lemma 1 of section 3.1, the result follows. 


\section{APPENDIX D}

SOME STANDARD NONLINEARITIES

In this appendix, some common nonlinearities will be listed together with their gains and sector bounds (defined in section 1.3). Conic bounds are not listed but can easily be derived from the sector bounds $(a, b)$ by

$$
\begin{aligned}
& \text { Centre of cone, } c=\frac{1}{2}(a+b) \\
& \text { Radius of cone, } r=\frac{1}{2}|b-a|
\end{aligned}
$$

The nonlinearity, $\mathbf{M}_{i}$ will be classified as invertible if a causal solution exists for all inputs, $x$ to the feedback system of Fig. 1 below. From section 4.2, the presence of a non-invertible nonlinearity implies that the design parameter, $Q\left(q^{-1}\right) \neq 0$ for well-posedness.

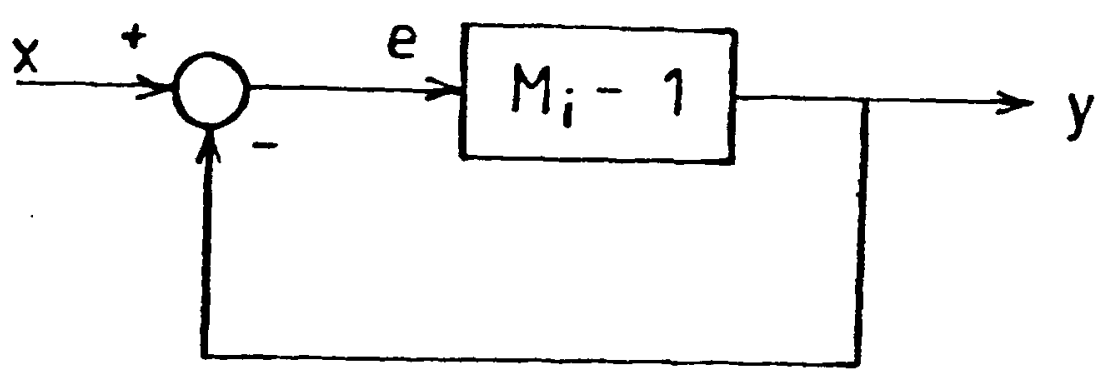

Fig. D-1 Invertible? 
Appendix D

In practice, mogt devices (including actuators and transducers) are only linear over some operating range. Where this is range is not adequate, the device can often be Iinearised to some extent. If the shape of the nonlinearity is roughly known eg. pH curves, an inverse nonlinearity may be included to compensate for it though considerable engineering effort has to be undertaken to ensure that it doesn't make it worsel. Alternatively, the device may be linearised by means of inner loop feedback. An example of this technique in use with a self-tuning controllex can be found in Hodgson and Clarke[96]. 

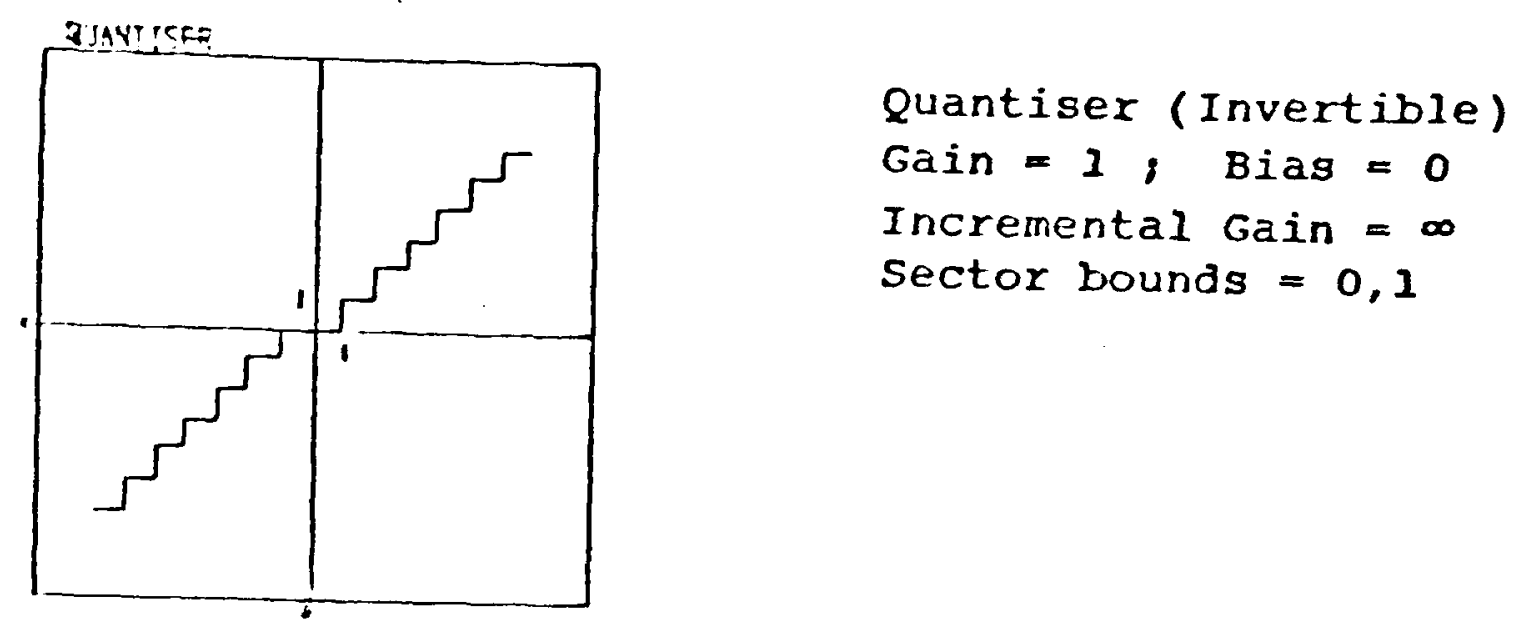

Relay with Hysterisis (Non-invertible) Gain = Incremental Gain $=\infty$

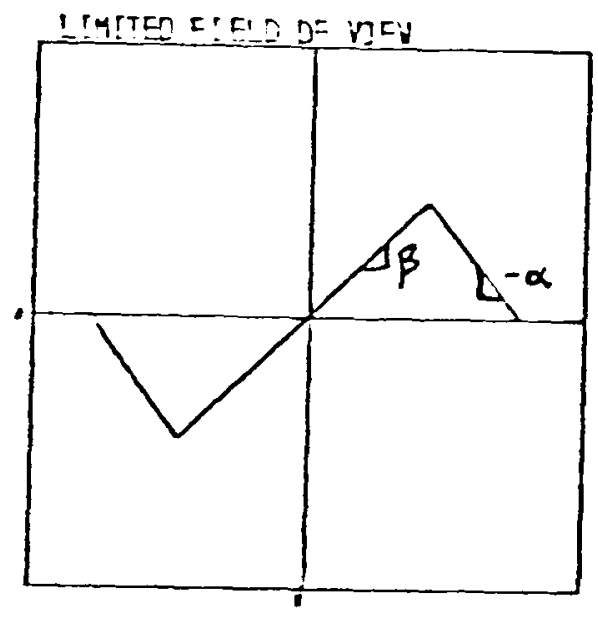

Limited Field of View (Non-invertible)

Gain $=$ larger of $\{\alpha, \beta\} ; B i a s=0$

Incremental Gain = Gain

sector bounds $=\alpha, \beta$ if $\beta, \alpha$ or vice versa

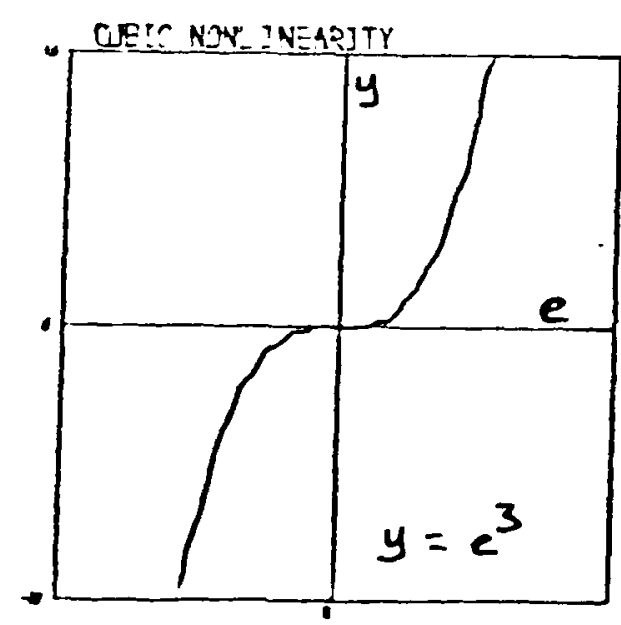

Cubic Eunction (Invertible)

Gain $=3.0 * \max |e|^{2}$ i.e. depends on region of operation

Bias $=0$

Incremental Gain = Gain

sector bounds $=0$, Gain 


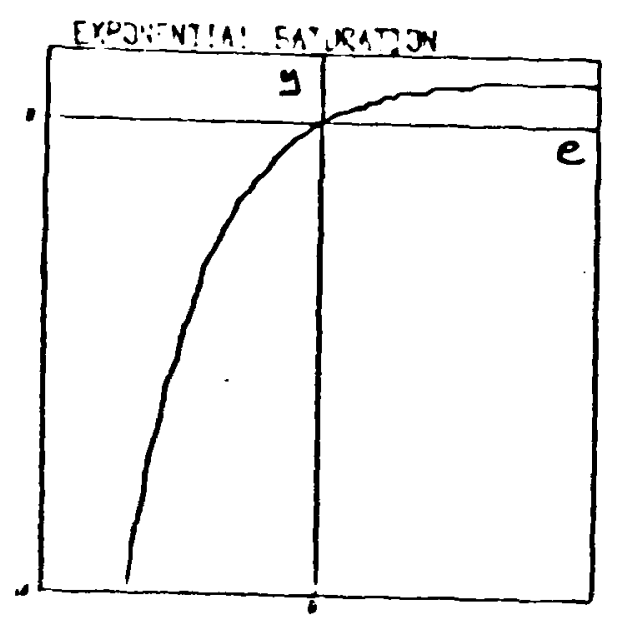

Exponential saturation (Invertible)

$(y=1.0-\exp [-c e])$

Gain $=c * \sup _{e} \exp [-c e]$ i.e. dependent on region of operation

Bias $=0$

Incremental Gain = Gain

sector bounds depend on region of operation

Biased Relay (Non-invertible)

Gain $=\beta / \alpha ;$ Bias $=\alpha$

Incremental Gain $=\infty$

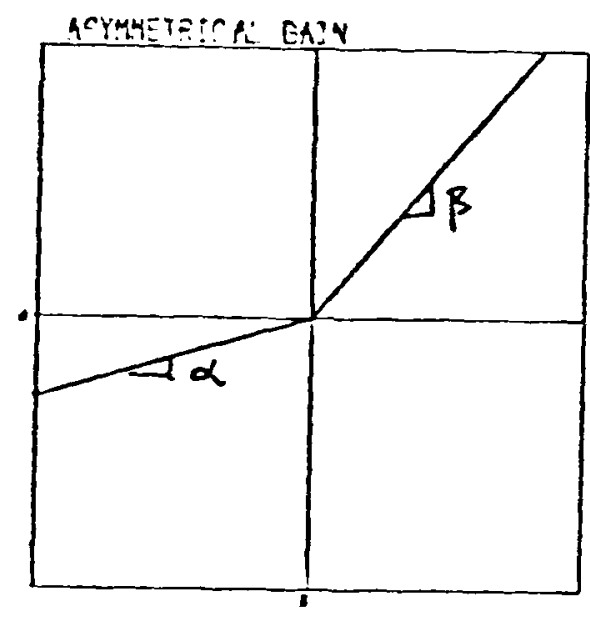

Asymmetrical Gain (Invertible)

Gain = larger of $\{\alpha, \beta\} ;$ Bias $=0$

Incremental Gain = Gain

sector bounds $=\alpha, \beta$ if $\beta$, $\alpha$ or vice versa

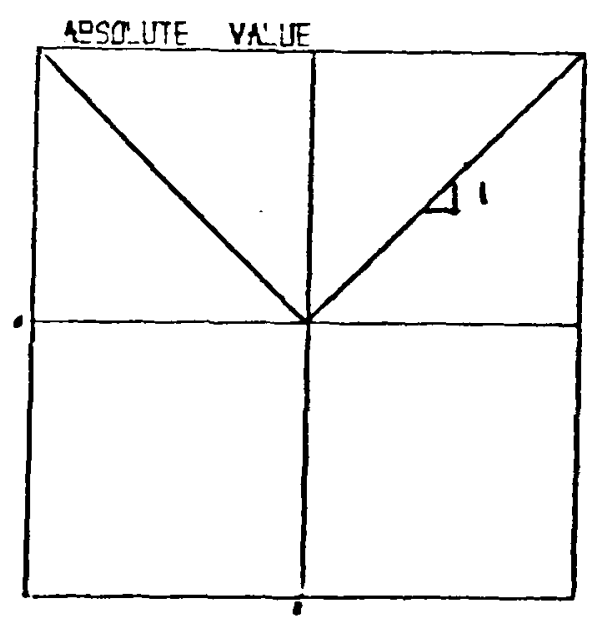

Absolute Value (Non-invertible)

Gain $=1$; Bias $=0$

Incremental Gain $=1$ 


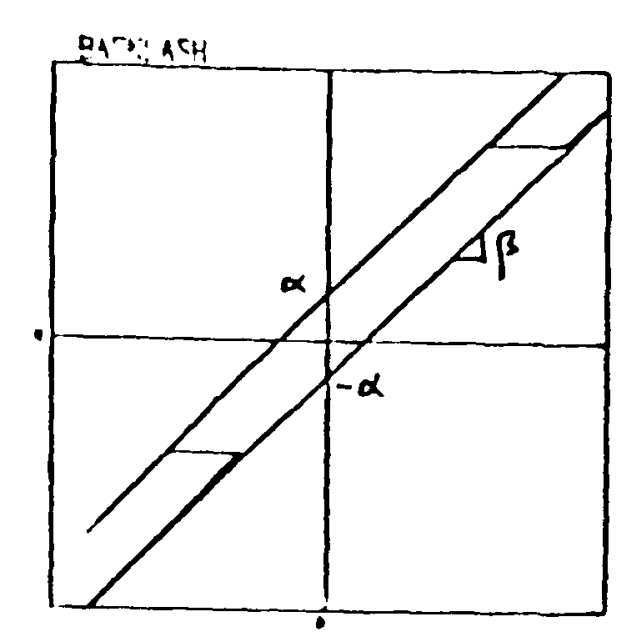

Backlash (Non-invertible)

Gain $=\boldsymbol{\beta} ;$ Biag $=\alpha$

Incremental Gain $=\beta$

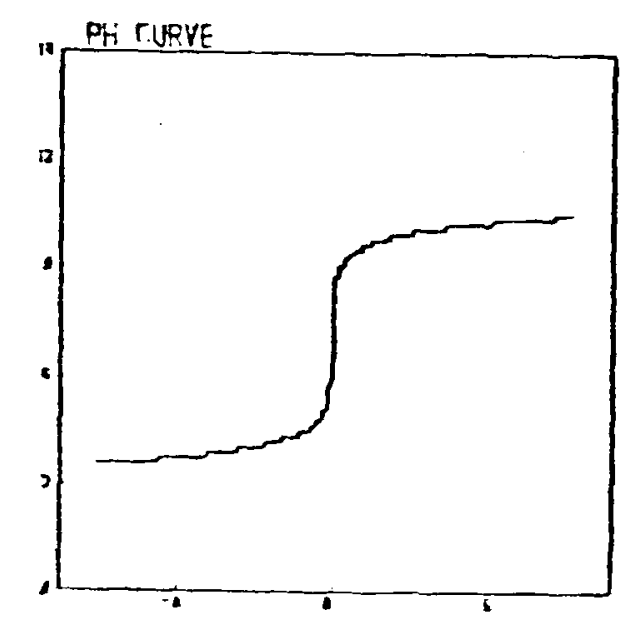

Ideal $\mathrm{pH}$ characteristic (Invertible)

Gain = very large $\left(\approx 10^{14}\right) ;$ Bias $=0$ Incremental Gain = Gain

sector bounds $=\infty 0, \infty 10^{14}$

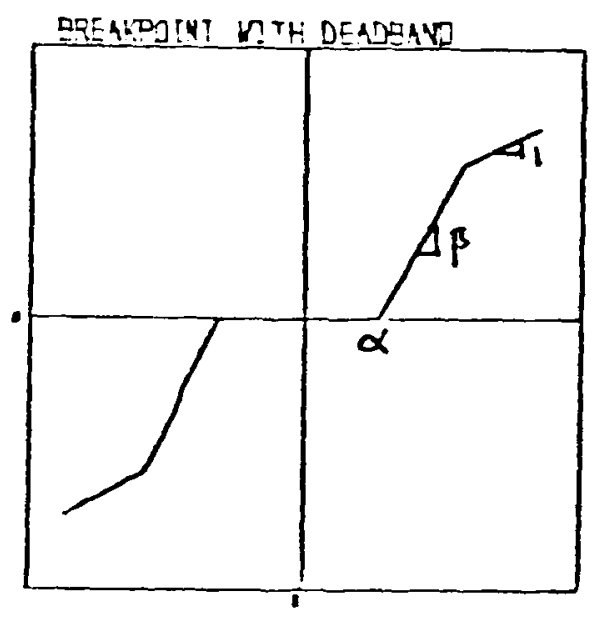

Breakpoint with Deadband (Invertible)

Gain $=\beta$ if $\beta>1$ or 1 if $\beta<1 ; \quad B i a s=\alpha$ Incremental Gain = as above

sector bounds $=0, \beta$ or 1

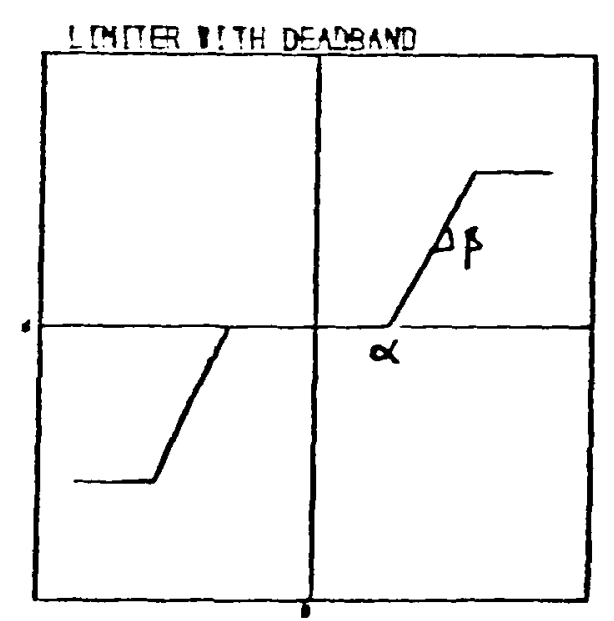

Limiter with Deadband (Non-invertible)

Gain $=\beta ;$ Bias $=\alpha$

Incremental Gain $=\beta$

Sector bounds $=0, \beta$ 


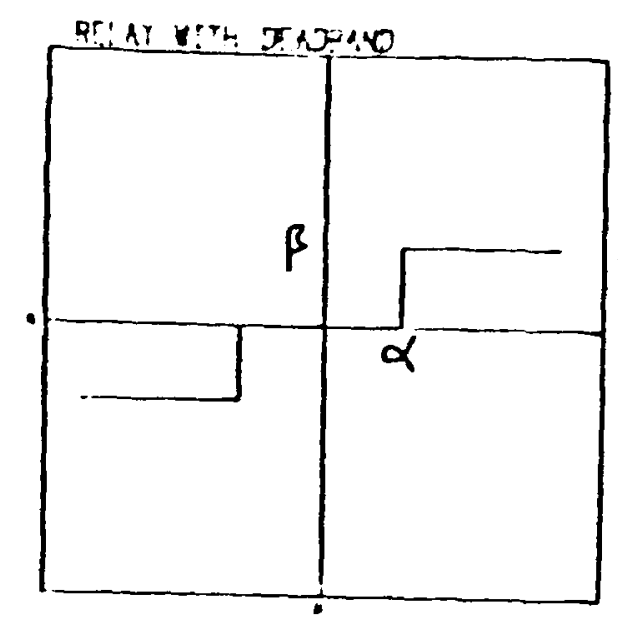

Relay with Deadband (Non-invertible) Gain $=\beta / \alpha, \quad$ Bias $=0$ Incremental Gain $=\infty$ sector bounds $=0, \beta / \alpha$
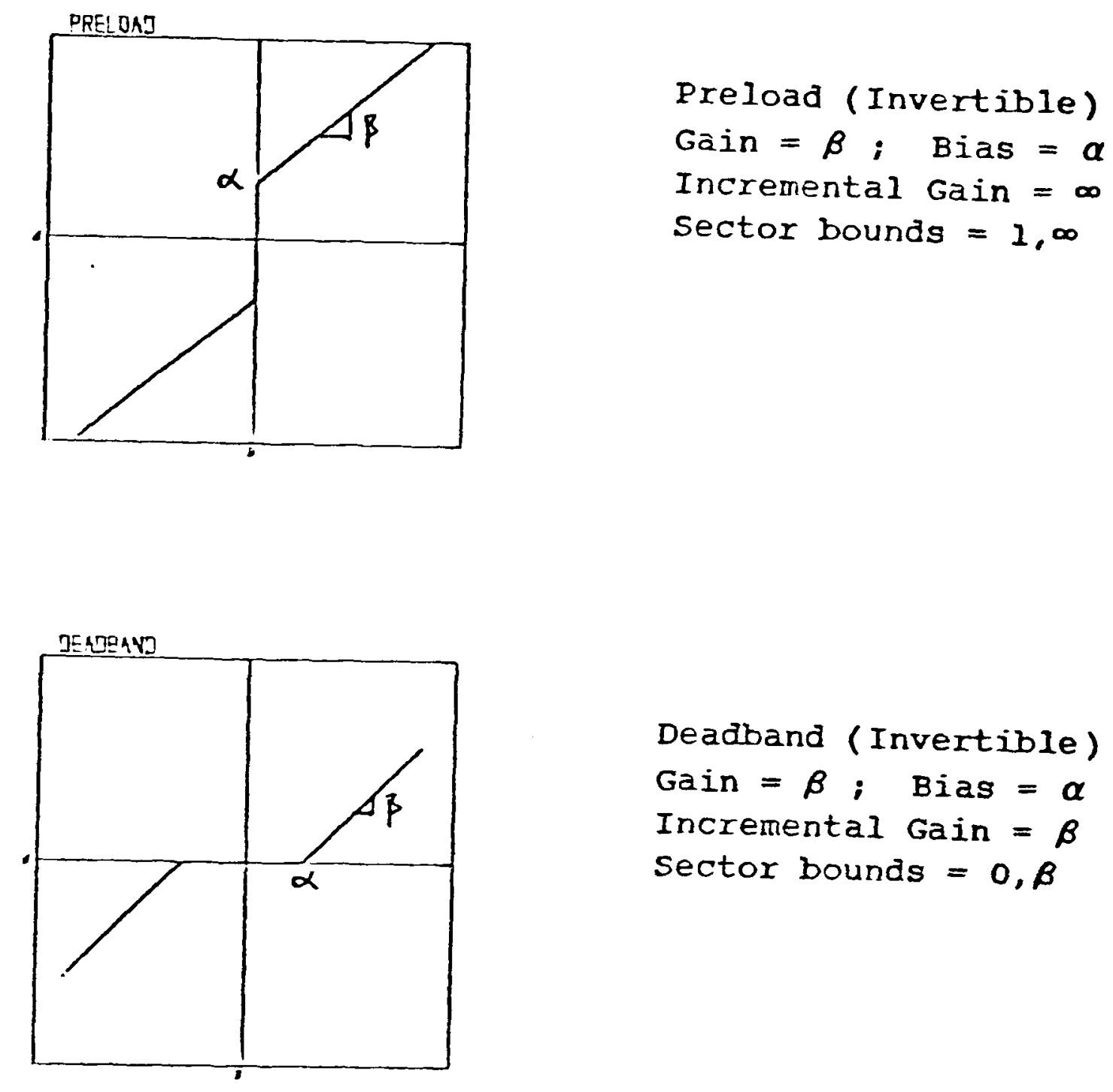

Deadband (Invertible)

Gain $=\beta ;$ Bias $=\alpha$

Incremental Gain $=\beta$

sector bounds $=0, \beta$

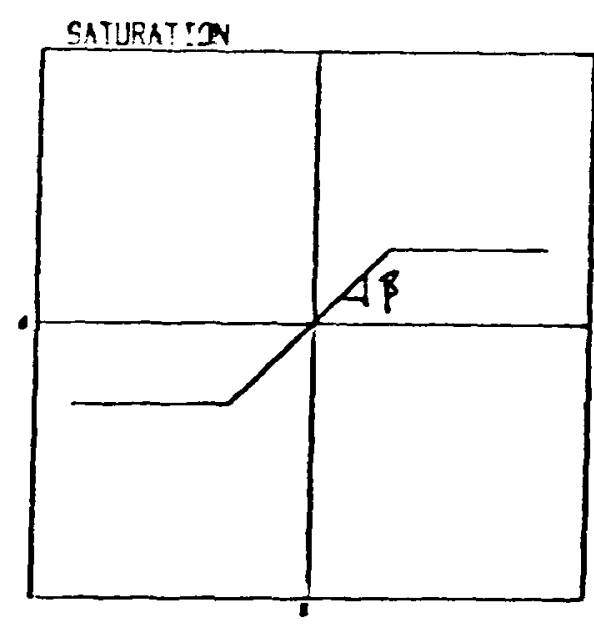

Saturation (Non-invertible)

Gain $=\beta ;$ Bias $=0$

Incremental Gain $=\beta$

Sector bounds $=0, \beta$ 
